\title{
ELLIPTIC STRUCTURES ON WEIGHTED THREE-DIMENSIONAL FANO HYPERSURFACES
}

\author{
IVAN CHELTSOV
}

\begin{abstract}
We classify birational maps into elliptic fibrations of a general quasismooth hypersurface in $\mathbb{P}\left(1, a_{1}, a_{2}, a_{3}, a_{4}\right)$ of degree $\sum_{i=1}^{4} a_{i}$ that has terminal singularities.
\end{abstract}

\section{INTRODUCTION.}

Let $X$ be a quasismooth hypersurface in $\mathbb{P}\left(1, a_{1}, a_{2}, a_{3}, a_{4}\right)$ of degree $d=\sum_{i=1}^{4} a_{i}$ that has terminal singularities, where $a_{1} \leqslant a_{2} \leqslant a_{3} \leqslant a_{4}$. Then the hypersurface $X$ is a Fano threefold, and there are exactly 95 possibilities for the four-tuple $\left(a_{1}, a_{2}, a_{3}, a_{4}\right)$. We use the notation $n$ for the entry numbers of these families, which are ordered in the same way as in [7].

Suppose that the hypersurface $X$ is general. The following result is proved in 6 ]

Theorem 1.1. The hypersurface $X$ is birationally rigid ${ }^{1}$.

For every $n$ there are involutions $\tau_{1}, \ldots, \tau_{k_{n}} \in \operatorname{Bir}(X)$ that generates the group $\operatorname{Bir}(X)$ up to biregular automorphisms (see [6]). In the case when $n \notin\{7,20,60\}$ and $k_{n}>0$, the hypersurface $X$ can be birationally transformed into an elliptic fibration that is invariant under the induced action of the group $\operatorname{Bir}(X)$, which is used in [4] to find relations between $\tau_{1}, \ldots, \tau_{k_{n}}$.

It is natural to try to classify all birational transformations of the hypersurface $X$ into elliptic fibrations, which is equivalent to the following problem: find all rational maps $X \rightarrow \mathbb{P}^{2}$ whose general fiber is birational to an elliptic curve. Let us consider few examples.

Example 1.2. Let $n=1$. Then $X$ is a quartic threefold. Let $\xi: X \rightarrow \mathbb{P}^{2}$ be a projection from a line that is contained in $X$. Then a general fiber of the map $\xi$ is an elliptic curve.

Example 1.3. Let $n=2$. Then $X$ is a hypersurface in $\mathbb{P}(1,1,1,1,2)$ of degree 5 , which has one singular point of type $\frac{1}{2}(1,1,1)$. There is a commutative diagram

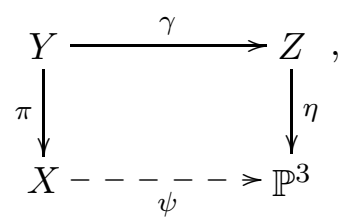

where $\psi$ is the natural projection, $\pi$ is a weighted blow up of the singular point of the hypersurface $X$ with weights $(1,1,1), \gamma$ is a birational morphism that contracts 15 irreducible smooth rational curves $C_{1}, \ldots, C_{15}$, and $\eta$ is a double cover. Put $\xi=\chi \circ \psi$, where $\chi: \mathbb{P}^{3} \rightarrow \mathbb{P}^{2}$ is a projection from the point $\eta \circ \gamma\left(C_{i}\right)$. Then a general fiber of the map $\xi$ is an elliptic curve.

Example 1.4. Let $n=17$. Then $X$ is a hypersurface in $\mathbb{P}(1,1,3,4,4)$ of degree 12 , whose singularities consist of three singular points of type $\frac{1}{4}(1,1,3)$. There is a commutative diagram

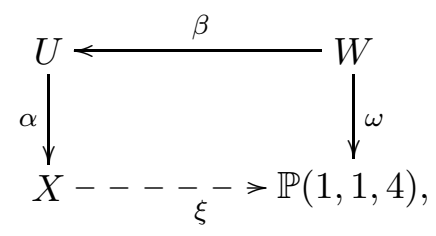

\footnotetext{
${ }^{1}$ Let $V$ be a Fano variety of Picard rank 1 having terminal $\mathbb{Q}$-factorial singularities. Then $V$ is said to be birationally rigid if it is not birational to any other Mori fiber space (see [3]). The variety $V$ is said to be birationally superrigid if it is birationally rigid and $\operatorname{Bir}(V)=\operatorname{Aut}(V)$.
} 
where $\xi$ is a projection, $\alpha$ is a weighted blow up of a singular point of type $\frac{1}{4}(1,1,3)$ with weights $(1,1,3), \beta$ is a weighted blow up with weights $(1,1,2)$ of the singular point that is contained in the exceptional divisor of the morphism $\alpha$, and $\omega$ is an elliptic fibration.

Example 1.5. Let $n=26$. Then $X$ is a hypersurface in $\mathbb{P}(1,1,3,5,6)$ of degree 15 that has two singular points of type $\frac{1}{3}(1,1,2)$. There is a commutative diagram

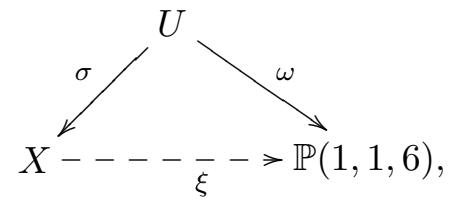

where $\xi$ is a projection, $\sigma$ is a weighted blow up of a singular point of type $\frac{1}{3}(1,1,2)$ with weights $(1,1,2)$, and $\omega$ is a morphism given by the linear system $\left|-6 K_{X}\right|$. Then the normalization of a general fiber of the rational map $\xi$ is an elliptic curve.

Example 1.6. Let $n=31$. Then $X$ is a hypersurface in $\mathbb{P}(1,1,4,5,6)$ of degree 16 that has a singular point of type $\frac{1}{5}(1,1,4)$, and there is a commutative diagram

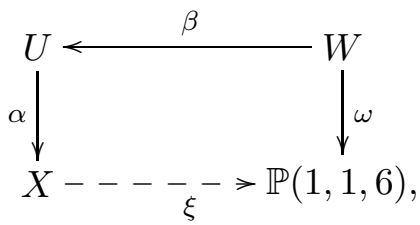

where $\xi$ is a projection, $\alpha$ is a weighted blow up of the singular point of type $\frac{1}{5}(1,1,4)$ with weights $(1,1,4), \beta$ is a weighted blow up with weights $(1,1,3)$ of the singular point that is contained in the exceptional divisor of $\alpha$, and $\omega$ is an elliptic fibration.

Example 1.7. Let $n \in\{7,11,19\}$. Then $a_{2}=a_{3}$, and the hypersurface $X$ has $d / a_{2}$ singular points of type $\frac{1}{a_{2}}\left(1,1, a_{2}-1\right)$. Let $\xi: X \rightarrow \mathbb{P}\left(1, a_{1}, a_{2}\right)$ be a rational map induced by a linear subsystem in the linear system $\left|-a_{2} K_{X}\right|$ consisting of surfaces that pass through a given singular points of type $\frac{1}{a_{2}}\left(1,1, a_{2}-1\right)$. Then the normalization of a general fiber of $\xi$ is an elliptic curve.

Example 1.8. Let $n \in\{7,9,20,30,36,44,49,51,64\}$. Then $X$ can be given by

$$
t z^{k}+\sum_{i=0}^{k-1} g_{i}(x, y, t, w) z^{i}=0 \text { or } w^{2} t+w g(x, y, z, t)+f(x, y, z, t)=0 \subset \operatorname{Proj}(\mathbb{C}[x, y, z, t, w]),
$$

where $\operatorname{wt}(x)=1, \operatorname{wt}(y)=a_{1}, \operatorname{wt}(z)=a_{2}, \operatorname{wt}(t)=a_{3}, \operatorname{wt}(w)=a_{4}$, and $g_{i}$ is a quasihomogeneous polynomial. Let $\xi: X \rightarrow \mathbb{P}\left(1, a_{1}, a_{3}\right)$ be a rational map given by a linear system consisting of surfaces that are cut out by $f(x, y, t)=0$, where $f(x, y, t)$ is a quasihomogeneous polynomial of degree $a_{3}$. Then the normalization of a general fiber of the map $\xi$ is an elliptic curve.

Example 1.9. Let $n \notin\{1,2,3,7,11,19,60,75,84,87,93\}$, and $\xi: X \rightarrow \mathbb{P}\left(1, a_{1}, a_{2}\right)$ be the natural projection. Then the normalization of a general fiber of the map $\xi$ is an elliptic curve.

The purpose of this paper is to prove the following result ${ }^{2}$.

Theorem 1.10. Let $\rho: X \rightarrow \mathbb{P}^{2}$ be a rational map whose general fiber is birational to an elliptic curve. Then there is a commutative diagram

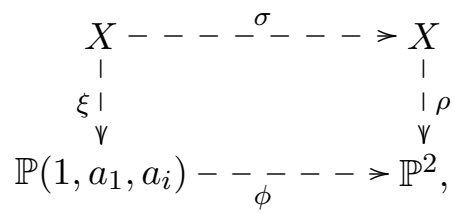

where $\phi$ is a birational map, $\sigma \in \operatorname{Bir}(X)$, and $\xi$ is one of the dominant rational maps constructed in Examples 1.2, 1.3, 1.4, 1.5, 1.6, 1.7, 1.8 and 1.9.

\footnotetext{
${ }^{2}$ In the case when $n \in\{1,3,14,22,28,34,37,39,52,53,57,59,60,66,70,72,73,75,78,81,84,86,87,88,89,90,92$, 93, 94, 95\}, the claim of Theorem 1.10 is proved in [1, 2, 4 .
} 
Corollary 1.11. Let $\rho: X \rightarrow \mathbb{P}^{2}$ be a rational map whose general fiber is birational to an elliptic curve. Suppose that $n \notin\{1,2,7,9,11,17,19,20,26,30,31,36,44,49,51,64\}$. Then there is a birational map $\psi: \mathbb{P}\left(1, a_{1}, a_{2}\right) \rightarrow \mathbb{P}^{2}$ such that the diagram

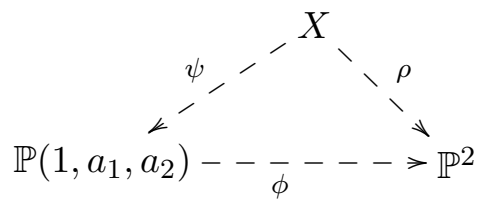

commutes, where $\psi$ is the natural projection.

Corollary 1.12. The hypersurface $X$ can be birationally transformed into an elliptic fibration if and only if $n \notin\{3,60,75,84,87,93\}$.

To illustrate our technique let us prove the following result.

Proposition 1.13. The claim of Theorem 1.10 holds for $n=14$.

Proof. Let $n=14$. Then $X$ is a hypersurface in $\mathbb{P}(1,1,1,4,6)$ of degree 12 , the singularities of the hypersurface $X$ consist of a singular point of type $\frac{1}{2}(1,1,1)$. Let $\psi: X \rightarrow \mathbb{P}^{2}$ be the natural projection, and $\pi: U \rightarrow X$ be a weighted blow up with weights $(1,1,1)$ of the singular point of the hypersurface $X$. Then $\psi \circ \pi$ is an elliptic fibration.

Let $\rho: X \rightarrow \mathbb{P}^{2}$ be a rational map such that the normalization of a general fiber of the rational map $\rho$ is an irreducible elliptic curve. Let us consider commutative diagram

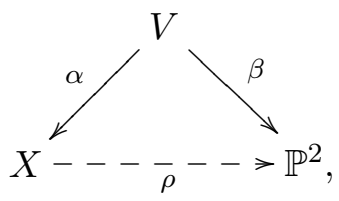

where $V$ is smooth, $\alpha$ is a birational morphism, and $\beta$ is a morphism. Let $\mathcal{M}$ be the proper transform of $\left|\beta^{*}\left(\mathcal{O}_{\mathbb{P}^{2}}(1)\right)\right|$ on $X$. There is a natural number $k>0$ such that $\mathcal{M} \sim-k K_{X}$. Then

$$
K_{V}+\frac{1}{k}\left|\beta^{*}\left(\mathcal{O}_{\mathbb{P}^{2}}(1)\right)\right| \sim_{\mathbb{Q}} \alpha^{*}\left(K_{X}+\frac{1}{k} \mathcal{M}\right)+\sum_{i=1}^{\delta} a_{i} E_{i} \sim_{\mathbb{Q}} \sum_{i=1}^{\delta} a_{i} E_{i},
$$

where $E_{i}$ is an $\alpha$-exceptional divisor, $a_{i}$ is a rational number, and $\delta$ is the number of exceptional divisors of the birational morphism $\alpha$. It follows from [6] that $a_{i} \geqslant 0$ for every $i$, but that there is an index $j$ such that $a_{j} \leqslant 0$ by Lemma 2.1. Put $Z_{j}=\alpha\left(E_{j}\right)$.

Suppose that $Z_{j}$ is a smooth point of $X$. Then $\operatorname{mult}_{Z_{j}}\left(S_{1} \cdot S_{2}\right) \geqslant 4 k^{2}$ by Lemma 1.10 in [5], but the linear system $\left|-4 K_{X}\right|$ induces a double cover $X \rightarrow \mathbb{P}(1,1,1,4)$. Thus, we have

$$
2 k^{2}=H \cdot S_{1} \cdot S_{2} \geqslant \operatorname{mult}_{Z_{j}}\left(S_{1} \cdot S_{2}\right) \geqslant 4 k^{2},
$$

where $S_{1}$ and $S_{2}$ are general surfaces of the linear system $\mathcal{M}$, and $H$ is a sufficiently general divisor in the linear system $\left|-4 K_{X}\right|$ that passes through the point $Z_{j}$, which is a contradiction.

It follows from Corollary 2.9 that $Z_{j}$ is not a curve, which implies that $Z_{j}$ is the unique singular point of the hypersurface $X$. Let $\mathcal{D}$ be the proper transform of the linear system $\mathcal{M}$ on the variety $U$. Then $\mathcal{D} \sim_{\mathbb{Q}}-k K_{U}$ by Theorem 2.2, which implies that $\mathcal{D}$ lies in the fibers of the elliptic fibration $\psi \circ \pi$, which concludes the proof.

Let us describe the structure of the paper. We consider auxiliary results in Section 2 the first steps of the proof of Theorem 1.10 is done in Section 3, where we also prove Theorem 1.10 in the case when $n \in\{1,3,5,11,14,22,28,34,37,39,52,53,57,59,60,66,70,72,73,75,78,81,84,86,87$, $88,89,90,92,93,94,95\}$. Then we prove Theorem 1.10 in all other cases.

The author is very grateful to Max-Plank-Institute für Mathematik at Bonn for the hospitality and excellent work conditions. The author would like to thank A. Corti, M. Grinenko, V. Iskovskikh, Yu. Manin, J. Park, Yu. Prokhorov, A. Pukhlikov, V. Shokurov and D. Stepanov for useful conversations. 


\section{Preliminaries.}

Let $X$ be a threefold having terminal $\mathbb{Q}$-factorial singularities, $\mathcal{M}$ be a linear system on the threefold such that $\mathcal{M}$ does not have fixed components, and $\lambda$ be an arbitrary non-negative rational number. In this section we consider technical results describing properties of the mobile ${ }^{3}$ log pair $(X, \lambda \mathcal{M})$, which are used in the proof of Theorem 1.10. As usual, the set of centers of canonical singularities of the mobile log pair $(X, \lambda \mathcal{M})$ is denoted as $\mathbb{C} \mathbb{S}(X, \lambda \mathcal{M})$.

Lemma 2.1. Let $\rho: X \rightarrow \mathbb{P}^{2}$ be a rational map whose general fiber is birational to an elliptic curve, and $\pi: V \rightarrow X$ be a resolution of the indeterminacies of $\rho$. Suppose that the $\mathcal{M}$ is a proper transform of the linear system $\left|\rho \circ \pi^{*}\left(\mathcal{O}_{\mathbb{P}^{2}}(1)\right)\right|$, the divisor $-K_{X}$ is nef and big, and the equivalence $K_{X}+\lambda \mathcal{M} \sim_{\mathbb{Q}} 0$ holds. Then the singularities of the log pair $(X, \lambda \mathcal{M})$ are not terminal.

Proof. See the proof of Theorem 1.4.4 in [3].

The following well-known result is proved in $[8]$.

Theorem 2.2. Let $O$ be a singular point of the threefold $X$ of type $\frac{1}{r}(1, a, r-a)$, where $a$ and $r$ are coprime natural numbers such that $r>a$, and mult $_{O}(\mathcal{M})$ be a rational number such that

$$
\mathcal{D} \sim_{\mathbb{Q}} \pi^{*}(\mathcal{M})-\operatorname{mult}_{O}(\mathcal{M}) G,
$$

where $\pi: U \rightarrow X$ is a weighted blow up of $O$ with weights $(1, a, r-a)$, $G$ is the exceptional divisor of $\pi, \mathcal{D}$ is a proper transform of $\mathcal{M}$ on the variety $U$. Suppose that $\mathbb{C} S(X, \lambda \mathcal{M})$ contains either the point $O$, or a curve passing through the point $O$. Then $\operatorname{mult}_{O}(\mathcal{M}) \geqslant 1 /(r \lambda)$.

In the proof of Theorem 2.2 implies the following result.

Lemma 2.3. Under the assumptions and notations of Theorem 2.2, suppose that the singularities of $(X, \lambda \mathcal{M})$ are canonical and $\mathbb{C} S(X, \lambda \mathcal{M})=\{O\}$, but $\mathbb{C} \mathbb{S}(U, \lambda \mathcal{D}) \neq \varnothing$. Then

- the set $\mathbb{C} \mathbb{S}(U, \lambda \mathcal{D})$ does not contain smooth points of the surface $G \cong \mathbb{P}(1, a, r-a)$,

- if the set $\mathbb{C} \mathbb{S}(U, \lambda \mathcal{D})$ contains a curve $L$, then $L \in\left|\mathcal{O}_{\mathbb{P}(1, a, r-a)}(1)\right|$, and every singular point of the surface $G$ is contained in the set $\mathbb{C S}(U, \lambda \mathcal{D})$.

Proof. We consider only the case when $r=5$ and $a=2$. Thus, we have $G \cong \mathbb{P}(1,2,3)$.

Let $P$ and $Q$ be singular points of $G$, and $L$ be the curve in $\left|\mathcal{O}_{\mathbb{P}(1,2,3)}(1)\right|$. Then $L$ passes through the points $P$ and $Q$, but $\operatorname{mult}_{O}(\mathcal{M})=1 /(5 \lambda)$ by Theorem 2.2 , which implies $\left.\mathcal{D}\right|_{G} \sim_{\mathbb{Q}} \lambda L$.

Suppose that the set $\mathbb{C S}(U, \lambda \mathcal{D})$ contains a subvariety $Z$ of subvariety $U$ that is different from the curve $L$ and the points $P$ and $Q$. Then $Z \subset G$.

Suppose that $Z$ is a point. Then the point $Z$ is smooth on the variety $U$, which implies the inequality $\operatorname{mult}_{Z}(\mathcal{D})>1 / \lambda$. Let $C$ be a general curve in the linear system $\left|\mathcal{O}_{\mathbb{P}(1,2,3)}(6)\right|$ that passes through the point $Z$. Then the curve $C$ is not contained in the base locus of the linear system $\mathcal{D}$. Hence, we have $1 / \lambda=C \cdot \mathcal{D} \geqslant \operatorname{mult}_{Z}(C) \operatorname{mult}_{Z}(\mathcal{D})>1 / \lambda$, which is a contradiction.

Therefore, the subvariety $Z$ is a curve. Then $\operatorname{mult}_{Z}(\mathcal{D}) \geqslant 1 / \lambda$. Let $C$ be a sufficiently general curve in the linear system $\left|\mathcal{O}_{\mathbb{P}(1,2,3)}(6)\right|$. Then

$$
\frac{1}{\lambda}=C \cdot \mathcal{D} \geqslant \operatorname{mult}_{Z}(\mathcal{D}) C \cdot Z \geqslant \frac{C \cdot Z}{\lambda},
$$

which implies that $C \cdot Z=1$. Hence, the curve $Z$ is contained in $\left|\mathcal{O}_{\mathbb{P}(1,2,3)}(1)\right|$.

Lemma 2.4. Let $C$ be a curve on $X$ such that $C \in \mathbb{C S}(X, \lambda \mathcal{M})$. Suppose that the complete linear system $\left|-m K_{X}\right|$ is base-point-free for some natural number $m>0$. Then $-K_{X} \cdot C \leqslant-K_{X}^{3}$.

Proof. Let $M_{1}$ and $M_{2}$ be general surfaces in $\mathcal{M}$. Then the inequalities

$$
\operatorname{mult}_{C}\left(M_{1} \cdot M_{2}\right) \geqslant \operatorname{mult}_{C}\left(M_{1}\right) \operatorname{mult}_{C}\left(M_{2}\right) \geqslant \frac{1}{\lambda^{2}}
$$

hold. Let $H$ be a general surface in $\left|-m K_{X}\right|$. Then

$$
\frac{-m K_{X}^{3}}{\lambda^{2}}=H \cdot M_{1} \cdot M_{2} \geqslant\left(-m K_{X} \cdot C\right) \operatorname{mult}_{C}\left(M_{1} \cdot M_{2}\right) \geqslant \frac{-m K_{X} \cdot C}{\lambda^{2}},
$$

which implies that $-K_{X} \cdot C \leqslant-K_{X}^{3}$.

\footnotetext{
${ }^{3}$ Elementary properties of mobile log pairs can be found in 3 .
} 
Lemma 2.5. Suppose that the linear system $\mathcal{M}$ is not composed from a pencil. Then there is no proper Zariski closed subset $\Sigma \subsetneq X$ such that

$$
\operatorname{Supp}\left(S_{1}\right) \cap \operatorname{Supp}\left(S_{2}\right) \subset \Sigma \subsetneq X,
$$

where $S_{1}$ and $S_{2}$ are general divisors in the linear system $\mathcal{M}$.

Proof. Suppose that there is a proper Zariski closed subset $\Sigma \subset X$ such that the set-theoretic intersection of the sufficiently general divisors $S_{1}$ and $S_{2}$ of the linear system $\mathcal{M}$ is contained in the set $\Sigma$. Let $\rho: X \rightarrow \mathbb{P}^{n}$ be a rational map induced by the linear system $\mathcal{M}$, where $n$ is the dimension of the linear system $\mathcal{M}$. Then there is a commutative diagram

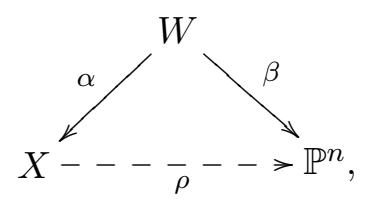

where $W$ is a smooth variety, $\alpha$ is a birational morphism, and $\beta$ is a morphism. Let $Y$ be the image of the morphism $\beta$. Then $\operatorname{dim}(Y) \geqslant 2$, because $\mathcal{M}$ is not composed from a pencil.

Let $\Lambda$ be a Zariski closed subset of the variety $W$ such that the morphism

$$
\left.\alpha\right|_{W \backslash \Lambda}: W \backslash \Lambda \longrightarrow X \backslash \alpha(\Lambda)
$$

is an isomorphism, and $\Delta$ be a union of the subset $\Lambda \subset W$ and the closure of the proper transform of the set $\Sigma \backslash \alpha(\Lambda)$ on the variety $W$. Then $\Delta$ is a Zariski closed proper subset of $W$.

Let $B_{1}$ and $B_{2}$ be general hyperplane sections of the variety $Y$, and $D_{1}$ and $D_{2}$ be proper transforms of the divisors $B_{1}$ and $B_{2}$ on the variety $W$ respectively. Then $\alpha\left(D_{1}\right)$ and $\alpha\left(D_{2}\right)$ are general divisors of the linear system $\mathcal{M}$. Hence, in the set-theoretic sense we have

$$
\varnothing \neq \beta^{-1}\left(\operatorname{Supp}\left(B_{1}\right) \cap \operatorname{Supp}\left(B_{2}\right)\right)=\operatorname{Supp}\left(D_{1}\right) \cap \operatorname{Supp}\left(D_{2}\right) \subset \Delta \subsetneq W,
$$

because $\operatorname{dim}(Y) \geqslant 2$. However, the set-theoretic identity [2.6] is an absurd.

The following result is implied by Lemma 0.3.3 in 9] and Lemma 2.5

Corollary 2.7. Let $X$ be a three-dimensional variety with canonical singularities, $D$ be divisor on the variety $X$ that is big and nef, $\mathcal{M}$ be a linear system on the variety $X$ that does not have fixed components and is not composed from a pencil, and $S_{1}$ and $S_{2}$ be sufficiently general surfaces of the linear system $\mathcal{D}$. Then the inequality $D \cdot S_{1} \cdot S_{2}>0$ holds.

The proof of Lemma 2.5 implies the following result.

Lemma 2.8. Let $X$ be a variety, $\mathcal{M}$ be a linear system on the variety $X$ that does not have fixed components and is not composed from a pencil, and $\mathcal{D}$ be a linear system on $X$ that does not have fixed components. Then there is no Zariski closed subset $\Sigma \subsetneq X$ such that

$$
\operatorname{Supp}(S) \cap \operatorname{Supp}(D) \subset \Sigma \subsetneq X,
$$

where $S$ and $D$ are sufficiently general divisors of the linear system $\mathcal{M}$ and $\mathcal{D}$ respectively.

The claim of Lemma 2.5 and the proof of Lemma 2.4 imply the following result.

Corollary 2.9. Under the assumptions and notations of Lemma 2.4, suppose that the linear system $\mathcal{M}$ is not composed from a pencil, and the divisor $-K_{X}$ is big. Then $-K_{X} \cdot C<-K_{X}^{3}$.

Many applications of Lemma 2.8 use the following simple result.

Lemma 2.10. Let $S$ be a surface, $D$ be an effective divisor on $S$ such that $D \equiv \sum_{i=1}^{r} a_{i} C_{i}$, where $a_{i} \in \mathbb{Q}$, and $C_{1}, \ldots, C_{r}$ are irreducible curves on $S$ whose intersection form is negatively defined. Then $D=\sum_{i=1}^{r} a_{i} C_{i}$.

Proof. Let $D=\sum_{i=1}^{k} c_{i} B_{i}$, where $B_{i}$ is an irreducible curve on $S$, and $c_{i}$ is a nonnegative rational number. Suppose that

$$
\sum_{i=1}^{k} c_{i} B_{i} \neq \sum_{i=1}^{r} a_{i} C_{i}
$$


and the curve $B_{i}$ is not one of the curves among $C_{1}, \ldots, C_{r}$ for every $i$. We have

$$
0 \geqslant\left(\sum_{a_{i}>0} a_{i} C_{i}\right) \cdot\left(\sum_{a_{i}>0} a_{i} C_{i}\right)=\left(\sum_{i=1}^{k} c_{i} B_{i}\right) \cdot\left(\sum_{a_{i}>0} a_{i} C_{i}\right)-\left(\sum_{a_{i} \leqslant 0} a_{i} C_{i}\right) \cdot\left(\sum_{a_{i}>0} a_{i} C_{i}\right) \geqslant 0
$$

which gives $\sum_{c_{i} \geqslant 0} c_{i} B_{i} \equiv \sum_{a_{i} \leqslant 0} a_{i} C_{i}$. Hence, we have $c_{i}=0$ and $a_{i}=0$ for every $i$.

\section{BEginNing of ClassifiCATION.}

Let us use the notations and assumptions of Section 11 In this section we begin to prove the claim of Theorem 1.10. Suppose that there is a birational map $\rho: X \rightarrow V$ and an elliptic fibration $\nu: V \rightarrow \mathbb{P}^{2}$ such that $V$ is smooth, and fibers of $\nu$ are connected. We must show that there is a commutative diagram

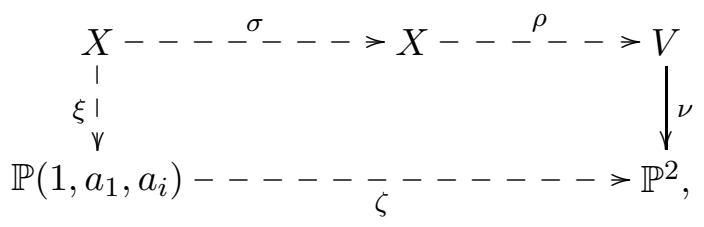

where $\phi$ is a birational map, $\sigma$ is a birational automorphism of $X$, and $\xi$ is one of the dominant rational maps constructed in Examples 1.2, 1.3, 1.4, 1.5, 1.6, 1.7, 1.8 and 1.9.

The commutative diagram 3.1 implies the commutative diagram

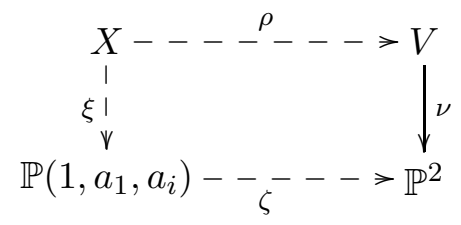

in the case when $\xi \circ \sigma=\chi \circ \xi$ for every $\sigma \in \operatorname{Bir}(X)$, where $\chi \in \operatorname{Bir}\left(\mathbb{P}\left(1, a_{1}, a_{i}\right)\right)$.

Example 3.3. Let $\psi: X \rightarrow \mathbb{P}\left(1, a_{1}, a_{2}\right)$ be a projection, and $\sigma$ be any birational automorphism of the threefold $X$. Suppose that $n \notin\{1,2,3,7,11,19,20,36,60,75,84,87,93\}$. Then it follows from [6] that there is a birational automorphism $\chi$ of $\mathbb{P}\left(1, a_{1}, a_{2}\right)$ such that $\psi \circ \sigma=\chi \circ \psi$.

Let $\mathcal{M}$ be a proper transform of the linear system $\left|\nu^{*}\left(\mathcal{O}_{\mathbb{P}^{2}}(1)\right)\right|$ on $X$. Then $\mathcal{M} \sim_{\mathbb{Q}}-k K_{X}$ for some natural $k$, but the singularities of the $\log$ pair $\left(X, \frac{1}{k} \mathcal{M}\right)$ are not terminal by Lemma 2.1.

Remark 3.4. It follows from [6] that there is a birational automorphism $\sigma \in \operatorname{Bir}(X)$ such that the singularities of the log pair $\left(X, \frac{1}{k^{\prime}} \sigma(\mathcal{M})\right)$ are canonical, where $k^{\prime} \in \mathbb{N}$ such that $\mathcal{M} \sim_{\mathbb{Q}}-k^{\prime} K_{X}$.

We may assume that the singularities of the $\log$ pair $\left(X, \frac{1}{k} \mathcal{M}\right)$ are canonical.

Theorem 3.5. The set $\mathbb{C S}\left(X, \frac{1}{k} \mathcal{M}\right)$ does not contain smooth points of $X$ if $n \neq 1$ and $n \neq 2$.

Proof. The claim follows from the proof of Theorem 5.1.2 in [6].

The following corollary is implied by Lemma 2.4

Corollary 3.6. The set of centers of canonical singularities $\mathbb{C S}\left(X, \frac{1}{k} \mathcal{M}\right)$ does not contain curves that do not contain singular points of the hypersurface $X$ in the case when $n \geqslant 6$.

The set $\mathbb{C} \mathbb{S}\left(X, \frac{1}{k} \mathcal{M}\right)$ contains a singular point of $X$ in the case $n \geqslant 6$ by Theorem 2.2

Proposition 3.7. Suppose that the set $\mathbb{C S}\left(X, \frac{1}{k} \mathcal{M}\right)$ contains a singular point $O$ of the hypersurface $X$ that is a singularity of type $\frac{1}{r}(1, r-a, a)$, where $a$ and $r$ are coprime natural numbers and $r>a$. Let $\pi: Y \rightarrow X$ be a weighted blow up of $O$ with weights $(1, a, r-a)$. Then $-K_{Y}^{3} \geqslant 0$.

Proof. Suppose that $-K_{Y}^{3}<0$. Let $E$ be the $\pi$-exceptional divisor, and $\mathcal{B}$ be a proper transform of $\mathcal{M}$ on the variety $Y$. Then $-K_{Y}^{3}=-K_{X}^{3}-1 /(r a(r-a))$, but $\mathcal{B} \sim_{\mathbb{Q}}-k K_{Y}$ by Theorem [2.2] 
Let $\overline{\mathbb{N E}}(Y)$ be a closure in $\mathbb{R}^{2}$ of the cone generated by effective one-dimensional cycles of the variety $Y$. Then $-E \cdot E$ generates an extremal ray of $\overline{\mathbb{N E}}(Y)$, but Corollary 5.4.6 in [6] implies that there are integers $b>0$ and $c \geqslant 0$ such that the cycle

$$
-K_{Y} \cdot\left(-b K_{Y}+c E\right)
$$

is numerically equivalent to an effective, irreducible and reduced curve $\Gamma$ on the variety $Y$ that generates the extremal ray of the cone $\overline{\mathbb{N E}}(Y)$ different from the ray generated by $-E \cdot E$.

Let $S_{1}$ and $S_{2}$ be general surfaces in $\mathcal{B}$. Then $S_{1} \cdot S_{2} \in \overline{\mathbb{N E}}(Y)$, but $S_{1} \cdot S_{2} \equiv k^{2} K_{Y}^{2}$, which implies that the cycle $S_{1} \cdot S_{2}$ generates an extremal ray of the cone $\overline{\mathbb{N E}}(Y)$ that contains the curve $\Gamma$. Moreover, for every effective cycle $C \in \mathbb{R}^{+} \Gamma$ we have

$$
\operatorname{Supp}(C)=\operatorname{Supp}\left(S_{1} \cdot S_{2}\right)
$$

because $S_{1} \cdot \Gamma<0$ and $S_{2} \cdot \Gamma<0$, which contradicts Lemma 2.5.

The following result is implied by Proposition 3.7

Proposition 3.8. The claim of Theorem 1.10 holds for $n \in\{14,22,28,34,37,39,52,53,57,59$, $66,70,72,73,78,81,86,88,89,90,92,94,95\}$.

Proof. We must show the existence of the commutative diagram

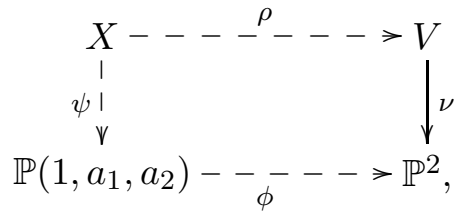

where $\psi$ is the natural projection, and $\phi$ is a birational map.

It follows from Theorems 2.2 and 3.5 and Lemma 2.4 that $\mathbb{C S}\left(X, \frac{1}{k} \mathcal{M}\right)$ contains a singular point $P \in X$ of type $\frac{1}{r}(1, a, r-a)$, where $a$ and $r$ are coprime natural numbers and $r>a$.

Let $\pi: Y \rightarrow X$ be a weighted blow up of $P$ with weights $(1, a, r-a)$, and $\mathcal{B}$ be the proper transform of the linear system $\mathcal{M}$ on variety $Y$. Then $-K_{Y}^{3}=0$ by Proposition 3.7

It is easy to see that the linear system $\left|-r K_{Y}\right|$ does not have base points for $r \gg 0$ and induces the morphism $\eta: Y \rightarrow \mathbb{P}\left(1, a_{1}, a_{2}\right)$ such that the diagram

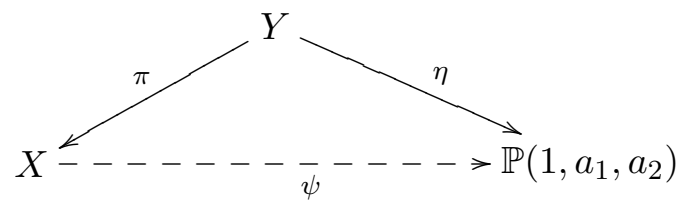

is commutative. The equivalence $\mathcal{B} \sim_{\mathbb{Q}}-k K_{Y}$ holds by Theorem 2.2, which implies the existence of the commutative diagram 3.9

The following result implies Corollary 1.12

Lemma 3.10. The claim of Theorem 1.10 holds for $n \notin\{3,60,75,84,87,93\}$.

Proof. It follows from Proposition 3.7 that $n \notin\{75,84,87,93\}$.

Suppose that $n=3$. Then the hypersurface $X$ is smooth, and the set $\mathbb{C} \mathbb{S}\left(X, \frac{1}{k} \mathcal{M}\right)$ contains an irreducible curve $\Gamma$ such that $-K_{X} \cdot \Gamma=1$ by Lemma 2.4. In particular, the curve $\Gamma$ is smooth.

Let $\gamma: \bar{X} \rightarrow X$ be a blow up of $\Gamma, G$ be the exceptional divisor of $\gamma$, and $\bar{S}_{1}$ and $\bar{S}_{2}$ are proper transforms on $\bar{X}$ of general surfaces in $\mathcal{M}$. Then the divisor $\gamma^{*}\left(-3 K_{X}\right)-G$ is nef and big, but

$$
\left(\eta^{*}\left(-3 K_{X}\right)-G\right) \cdot \bar{S}_{1} \cdot \bar{S}_{1}=0
$$

which contradicts Corollary 2.7

We have $n=60$. The threefold $X$ is a hypersurface in $\mathbb{P}(1,4,5,6,9)$ of degree 24 .

It is easy to check that $\mathbb{C} \mathbb{S}\left(X, \frac{1}{k} \mathcal{M}\right)$ does not contain curves by Corollary 2.9, which implies that the set $\mathbb{C S}\left(X, \frac{1}{k} \mathcal{M}\right)$ consists of the singular point $O$ of the hypersurface $X$ that is a quotient singularity of type $\frac{1}{9}(1,4,5)$ by Proposition 3.7. Let $\pi: Y \rightarrow X$ be a weighted blow up of the 
singular point $O$ with weights $(1,4,5)$, and $\mathcal{D}$ be a proper transform of the linear system $\mathcal{M}$ on the threefold $Y$. Then $\mathcal{D} \sim_{\mathbb{Q}}-k K_{Y}$ by Theorem 2.2 .

Let $P$ and $Q$ be the points of $Y$ contained in the $\pi$-exceptional divisor that are singularities of types $\frac{1}{4}(1,1,3)$ and $\frac{1}{5}(1,1,4)$ respectively. Then $\mathbb{C S}\left(Y, \frac{1}{k} \mathcal{D}\right) \subseteq\{P, Q\}$ by Lemmas 2.3 and 2.4.

Suppose that the set $\mathbb{C S}\left(Y, \frac{1}{k} \mathcal{D}\right)$ contains the point $Q$. Let $\alpha: U \rightarrow Y$ be a weighted blow up of the point $Q$ with weights $(1,1,4)$, and $\mathcal{B}$ be a proper transform of the linear system $\mathcal{M}$ on the variety $U$. Then $\mathcal{B} \sim_{\mathbb{Q}}-k K_{U}$ by Theorem 2.2 , the linear system $\left|-4 K_{U}\right|$ is a proper transform of the pencil $\left|-4 K_{X}\right|$, and the base locus of the pencil $\left|-4 K_{U}\right|$ consists of an irreducible reduced curve $Z$ on the variety $U$ such that the curve $\pi \circ \alpha(Z)$ is a base curve of the pencil $\left|-4 K_{X}\right|$.

Let $H$ be a general surface in $\left|-4 K_{U}\right|$. Then $Z^{2}=-1 / 30$ on $H$, but $\left.\mathcal{B}\right|_{H} \sim_{\mathbb{Q}} k Z$, which contradicts Lemmas 2.8 and 2.10. We see that $\mathbb{C S}\left(Y, \frac{1}{k} \mathcal{D}\right)=\{P\}$ by Lemmas 2.1] and 2.3.

Let $\beta: W \rightarrow Y$ be a weighted blow up of the point $P$ with weights $(1,1,3)$, and $D$ be the proper transform of the general surface in $\left|-5 K_{X}\right|$ on $W$. Then $D$ is nef and big, but the equality $D \cdot H_{1} \cdot H_{2}=0$ holds, where $H_{1}$ and $H_{2}$ are general surfaces of the proper transform of the linear system $\mathcal{M}$ on the variety $W$, which contradicts Corollary 2.7

Now we prove the following very simple result.

Proposition 3.11. Suppose that $n=11$. Then the diagram 3.2 exists, where $\xi$ is one of the five rational maps constructed in Example 1.7.

Proof. The threefold $X$ is a hypersurface in $\mathbb{P}(1,1,2,2,5)$ of degree 10 , whose singularities consist of points $P_{1}, P_{2}, P_{3}, P_{4}$ and $P_{5}$ that are singularities of types $\frac{1}{2}(1,1,1)$. The diagram

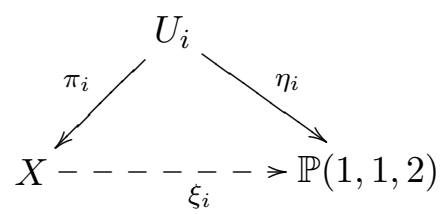

commutes, where $\xi_{i}$ is a projection, $\pi_{i}$ is the weighted blow up of $P_{i}$ with weights $(1,1,1)$, and $\eta_{i}$ is an elliptic fibration. It follows from Theorems 2.2 and 3.5 and Lemma 2.4 that $P_{i} \in \mathbb{C S}\left(X, \frac{1}{k} \mathcal{M}\right)$ for some $i \in\{1,2,3,4,5\}$.The diagram 3.2 exists for $\xi=\xi_{i}$ by Theorem 2.2

The following result is due to 12 .

Theorem 3.12. Suppose that $\mathbb{C} S\left(X, \frac{1}{k} \mathcal{M}\right)$ contains an irreducible curve $C$, and $n \geqslant 3$. Then

$$
\operatorname{Supp}(C) \subset \operatorname{Supp}\left(S_{1} \cdot S_{2}\right) \text {, }
$$

where $S_{1}$ and $S_{2}$ are different surfaces of the linear system $\left|-K_{X}\right|$.

Let us prove the following result of Daniel Ryder.

Proposition 3.13. The claim of Theorem 1.10 holds for $n=5$.

Proof. Let $n=5$. Then $X$ is a hypersurface in $\mathbb{P}(1,1,1,2,3)$ of degree 7 , whose singularities consist of the points $P$ and $Q$ such that $P$ is a singular point of type $\frac{1}{2}(1,1,1)$, and $Q$ is a singular point of type $\frac{1}{3}(1,1,2)$. The hypersurface $X$ can be given by the equation

$$
w^{2} f_{1}(x, y, z)+f_{4}(x, y, z, t) w+f_{7}(x, y, z, t)=0 \subset \mathbb{P}(1,1,1,2,3) \cong \operatorname{Proj}(\mathbb{C}[x, y, z, t, w]),
$$

where $\operatorname{wt}(x)=\operatorname{wt}(y)=\operatorname{wt}(z)=1, \operatorname{wt}(t)=2, \operatorname{wt}(w)=3$, and $f_{i}$ is a quasihomogeneous polynomial of degree $i$. There is a commutative diagram

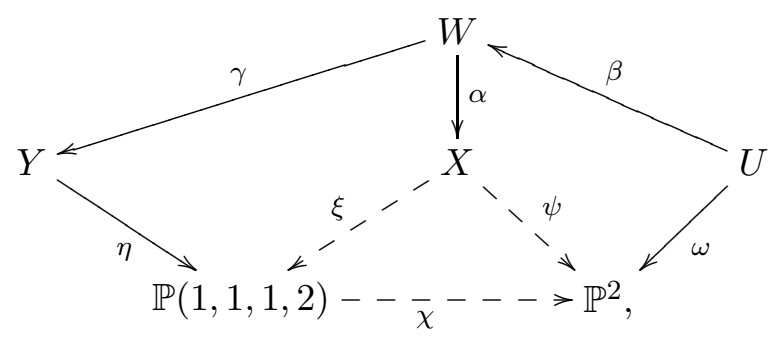


where $\chi$ and $\xi$ are the natural projections, the morphism $\omega$ is an elliptic fibration, the morphism $\alpha$ is a weighted blow up of the point $Q$ with weights $(1,1,2)$, the morphism $\gamma$ is the birational morphism that contracts 14 smooth irreducible rational curves $C_{1}, \ldots, C_{14}$ into 14 isolated ordinary double points $P_{1}, \ldots, P_{14}$ of the variety $Y$ respectively, the morphism $\eta$ is a double cover branched over the surface $R \subset \mathbb{P}(1,1,1,2)$ that is given by the equation

$$
f_{4}(x, y, z, t)^{2}-4 f_{1}(x, y, z) f_{7}(x, y, z, t)=0 \subset \mathbb{P}(1,1,1,2) \cong \operatorname{Proj}(\mathbb{C}[x, y, z, t])
$$

and has 14 isolated ordinary double points $\eta\left(P_{1}\right), \ldots, \eta\left(P_{14}\right)$, and $\beta$ is the composition of the weighted blow ups with the weights $(1,1,1)$ of two singular points of the variety $W$ that are singularities of types $\frac{1}{2}(1,1,1)$. It follows from Theorem 3.12 that the set $\mathbb{C} \mathbb{S}\left(X, \frac{1}{k} \mathcal{M}\right)$ does not contain curves (see the proof of Lemma 8.3).

Suppose that the set $\mathbb{C S}\left(X, \frac{1}{k} \mathcal{M}\right)$ consists of the point $Q$. Let $O$ be the singular point of the threefold $W$ that dominates the singular point $Q$, and $\mathcal{D}$ be the proper transform of the linear system $\mathcal{M}$ on the threefold $W$. Then $O \in \mathbb{C S}\left(W, \frac{1}{k} \mathcal{D}\right)$ by Theorem 2.2 and Lemmas 2.1] and 2.3.

Let $\mathcal{B}$ be the proper transform of $\mathcal{D}$ on $Y$. Then it follows from Theorem 2.2 and Lemmas 2.3 and 2.4 that $\mathbb{C S}\left(Y, \frac{1}{k} \mathcal{B}\right)$ contains a curve $C$ such that $-K_{Y} \cdot C=1 / 2$, and $\chi \circ \eta(C)$ is a point.

There is an irreducible curve $Z$ on the variety $Y$ such that the curve $Z$ is different from the curve $C$, but $\eta(Z)=\eta(C)$. Let $S$ be a general surface of the linear system $\left|-K_{Y}\right|$ that contains the curve $C$. Then $Z^{2}<0$ on $S$, but $\left.\mathcal{B}\right|_{S} \sim_{\mathbb{Q}} k C+k Z$, which contradicts to Lemma 2.8.

It follows from Theorem 2.2 and Lemmas 2.3 and 2.4 that

$$
\mathbb{C} \mathbb{S}\left(X, \frac{1}{k} \mathcal{M}\right)=\{P, Q\},
$$

but $O \in \mathbb{C} \mathbb{S}\left(W, \frac{1}{k} \mathcal{D}\right)$ by Corollary 2.9 and Lemmas 2.1 and 2.3. Hence, the proper transform of the linear system $\mathcal{M}$ on the variety $U$ is contained in the fibers of $\omega$ by Theorem 2.2.

The claim of Theorem 3.12 implies the following result.

Lemma 3.14. Suppose that $a_{2} \neq 1$. Then the set $\mathbb{C} \mathbb{S}\left(X, \frac{1}{k} \mathcal{M}\right)$ does not contains curves.

Proof. Suppose that $\mathbb{C S}\left(X, \frac{1}{k} \mathcal{M}\right)$ contains a curve $C$. Then the claim of Theorem 3.12 implies that there are surfaces $S_{1}$ and $S_{2}$ of the pencil $\left|-K_{X}\right|$ such that the curve $C$ is an irreducible component of the irreducible and reduced curve $S_{1} \cap S_{2}$, which contradicts Lemma 2.4.

Let us illustrate Lemma 3.14 by proving the following result.

Proposition 3.15. The claim of Theorem 1.10 holds for $n=18$.

Proof. Let $n=18$. Then $X$ is a hypersurface in $\mathbb{P}(1,2,2,3,5)$ of degree 12 , whose singularities consist of the points $O_{1}, O_{2}, O_{3}, O_{4}, O_{5}$ and $O_{6}$ that are quotient singularities of type $\frac{1}{2}(1,1,1)$, and the point $P$ that is a quotient singularity of type $\frac{1}{5}(1,2,3)$. There is a commutative diagram

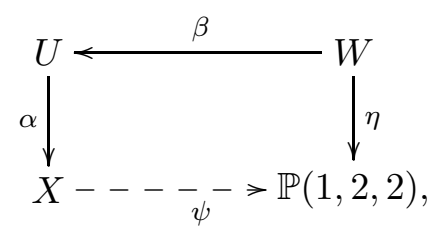

where $\psi$ is a projection, $\alpha$ is the weighted blow up of $P$ with weights $(1,2,3), \beta$ is the weighted blow up with weights $(1,1,2)$ of the singular point of the variety $U$ that is a quotient singularity of type $\frac{1}{3}(1,1,2)$, and $\eta$ is an elliptic fibration.

It follows from Theorem 3.5] Lemma 3.14 and Proposition 3.7 that $\mathbb{C S}\left(X, \frac{1}{k} \mathcal{M}\right)=\{P\}$.

Let $\mathcal{D}$ be the proper transform of $\mathcal{M}$ on the variety $U$, and $Q$ and $O$ be the singular points of the variety $U$ contained in the exceptional divisor of the birational morphism $\alpha$ that are singularities of types $\frac{1}{3}(1,1,2)$ and $\frac{1}{2}(1,1,1)$ respectively. Then $\mathcal{D} \sim_{\mathbb{Q}}-k K_{U}$ and

$$
\varnothing \neq \mathbb{C} S\left(U, \frac{1}{k} \mathcal{D}\right) \subseteq\{Q, O\}
$$

by Theorem 2.2 and Lemma 2.1, 2.3 and 2.4 
Suppose that the set $\mathbb{C S}\left(U, \frac{1}{k} \mathcal{D}\right)$ contains the point $O$. Let $\pi: Y \rightarrow U$ be the weighted blow up of $O$ with weights $(1,1,1), F$ be the $\pi$-exceptional divisor, and $\mathcal{H}$ and $\mathcal{P}$ be the proper transforms of $\mathcal{M}$ and $\left|-3 K_{U}\right|$ on $Y$ respectively. Then $\mathcal{H} \sim_{\mathbb{Q}}-k K_{Y}$ by Theorem 2.2, but

$$
\mathcal{P} \sim_{\mathbb{Q}} \pi^{*}\left(-3 K_{U}\right)-\frac{1}{2} F,
$$

and the base locus of the linear system $\mathcal{P}$ consists of the irreducible curve $Z$ such that $\alpha \circ \pi(Z)$ is the base curve of the linear system $\left|-3 K_{X}\right|$. Moreover, for a general surface $S$ of the linear system $\mathcal{P}$, the inequality $S \cdot Z>0$ holds, which implies that the divisor $\pi^{*}\left(-6 K_{U}\right)-F$ is nef and big. On the other hand, for general surfaces $D_{1}$ and $D_{2}$ of the linear system $\mathcal{H}$, we have

$$
\left(\pi^{*}\left(-6 K_{U}\right)-F\right) \cdot D_{1} \cdot D_{2}=\left(\pi^{*}\left(-6 K_{U}\right)-F\right) \cdot\left(\pi^{*}\left(-k K_{U}\right)-\frac{k}{2} F\right)^{2}=0,
$$

which contradicts Corollary 2.7

Therefore, the set $\mathbb{C} S\left(U, \frac{1}{k} \mathcal{D}\right)$ contains the point $Q$. Let $\mathcal{B}$ be the proper transform of the linear system $\mathcal{M}$ on the variety $W$. Then the equivalence $\mathcal{B} \sim_{\mathbb{Q}}-k K_{W}$ holds by Theorem 2.2 which easily implies that the claim of Theorem 1.10 holds for the hypersurface $X$.

We conclude this section by proving the following result, which is proved in [1] and [2].

Theorem 3.16. The claim of Theorem 1.10 holds for $n=1$.

Proof. Let $X$ be a general hypersurface in $\mathbb{P}^{4}$ of degree 4 . Then we must show that there is a line $L \subset X$ such that there is a commutative diagram

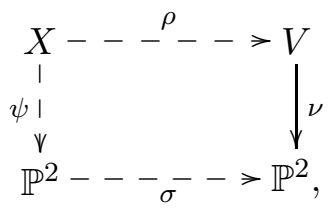

where $\psi$ is the projection from the $L$, and $\sigma$ is a birational map.

Suppose that $\mathbb{C} \mathbb{S}\left(X, \frac{1}{k} \mathcal{M}\right)$ contains a point $P$ of the quartic $X$. Let $H$ be a general hyperplane section of $X$ passing through the point $P$. Then it follows from Lemma 1.10 in [5] that

$$
4 k^{2} \leqslant \operatorname{mult}_{P}\left(D_{1} \cdot D_{2}\right) \leqslant D_{1} \cdot D_{2} \cdot H=4 k^{2},
$$

where $D_{1}$ and $D_{2}$ are general surfaces of the linear system $\mathcal{M}$. Therefore, the support of the effective one-dimensional cycle $D_{1} \cdot D_{2}$ is contained in the union of a finite number of lines on the quartic $X$ that pass through the point $P$, which contradicts Lemma 2.5.

Therefore, the set $\mathbb{C S}\left(X, \frac{1}{k} \mathcal{M}\right)$ contains a curve $C$. Thus, the inequality $\operatorname{mult}_{C}(\mathcal{M}) \geqslant k$ holds, but it follows from Lemma 2.4 that $\operatorname{deg}(C) \leqslant 3$.

Suppose that $C$ is not contained in any plane in $\mathbb{P}^{4}$. Then the curve $C$ is either a smooth curve of degree 3 or 4 , or a rational curve of degree 4 having one double point.

Suppose that $C$ is smooth. Let $\alpha: U \rightarrow X$ be the blow up of the curve $C, F$ be the the exceptional divisor of $\alpha$, and $\mathcal{D}$ be the proper transform of $\mathcal{M}$ on the variety $U$. Then the base locus of the linear system $\left|\alpha^{*}\left(-\operatorname{deg}(C) K_{X}\right)-F\right|$ does not contain curves, but

$$
\left(\alpha^{*}\left(-\operatorname{deg}(C) K_{X}\right)-F\right) \cdot D_{1} \cdot D_{2}<0,
$$

where $D_{1}$ and $D_{2}$ are general surfaces of the linear system $\mathcal{D}$, which is a contradiction.

Thus, the curve $C$ is a quartic curve with a double point $P$. Let $\beta: W \rightarrow X$ be a composition of the blow up of $P$ with the blow up of he proper transform of $C$. Let $G$ and $E$ be the exceptional divisors of $\beta$ such that $\beta(E)=C$ and $\beta(G)=P$. Then the base locus of the linear system $\left|\beta^{*}\left(-4 K_{X}\right)-E-2 G\right|$ does not contain curves, but

$$
\left(\beta^{*}\left(-4 K_{X}\right)-E-2 G\right) \cdot D_{1} \cdot D_{2}<0
$$

where $D_{1}$ and $D_{2}$ are general surfaces of the linear system $\mathcal{D}$, which is a contradiction.

Hence, we see that the curve $C$ is contained in a plane in $\mathbb{P}^{4}$.

Suppose that $\operatorname{deg}(C) \neq 1$. Then we have the following possibilities:

- the curve $C$ is a smooth conic; 
- the curve $C$ is a smooth plane cubic;

- the curve $C$ is a singular plane cubic.

Suppose that $C$ is smooth. Let $\alpha: U \rightarrow X$ be a blow up of the curve $C, F$ be the exceptional divisor of the morphism $\alpha$, and $\mathcal{D}$ be the proper transform of $\mathcal{M}$ on the variety $U$. Then one can easily check that the base locus of the linear system $\left|\alpha^{*}\left(-\operatorname{deg}(C) K_{X}\right)-F\right|$ does not contain curves. Therefore, the divisor $\alpha^{*}\left(-\operatorname{deg}(C) K_{X}\right)-F$ is nef and big, but

$$
\left(\alpha^{*}\left(-\operatorname{deg}(C) K_{X}\right)-F\right) \cdot D_{1} \cdot D_{2}=0,
$$

where $D_{1}$ and $D_{2}$ are general surfaces of $\mathcal{D}$, which contradicts Corollary 2.7

Hence, the curve $C$ is a plane cubic with a double point $P$. Let $\beta: W \rightarrow X$ be a composition of the blow up of $P$ with the blow up of the proper transform of $C$. Let $G$ and $E$ be the exceptional divisors of the morphism $\beta$ such that $\beta(E)=C$ and $\beta(G)=P$. Then the base locus of the linear system $\left|\beta^{*}\left(-3 K_{X}\right)-E-2 G\right|$ does not contain curves, which implies that the divisor $\beta^{*}\left(-3 K_{X}\right)-E-2 G$ is nef and big. On the other hand, the inequality

$$
\left(\beta^{*}\left(-3 K_{X}\right)-E-2 G\right) \cdot D_{1} \cdot D_{2} \leqslant 0
$$

holds, where $D_{1}$ and $D_{2}$ are general surfaces of $\mathcal{D}$, which is impossible by Corollary 2.7

Thus, we see that the curve $C$ is a line. The equality $\operatorname{mult}_{C}(D)=k$ implies the existence of the commutative diagram 3.17 where $L=C$.

\section{Case $n=2$, hypersurface of Degree 5 IN $\mathbb{P}(1,1,1,1,2)$.}

We use the notations and assumptions of Section 3 Let $n=2$. Then $X$ is a sufficiently general hypersurface in $\mathbb{P}(1,1,1,1,2)$ of degree 5 , the equality $-K_{X}^{3}=5 / 2$ holds, and the singularities of the hypersurface $X$ consist of a point $O$ that is a quotient singularity of type $\frac{1}{2}(1,1,1)$.

The hypersurface $X$ can be given by the equation

$$
w^{2} f_{1}(x, y, z, t)+f_{3}(x, y, z, t) w+f_{5}(x, y, z, t)=0 \subset \mathbb{P}(1,1,1,1,2) \cong \operatorname{Proj}(\mathbb{C}[x, y, z, t, w]),
$$

where $\operatorname{wt}(x)=\operatorname{wt}(y)=\operatorname{wt}(z)=\operatorname{wt}(t)=1$ and $\operatorname{wt}(w)=2, f_{i}(x, y, z, t)$ is a homogeneous polynomial of degree $i$, and the point $O$ is given by the equations $x=y=z=t=0$.

Let $\psi: X \rightarrow \mathbb{P}^{3}$ be the natural projection. Then there is a commutative diagram

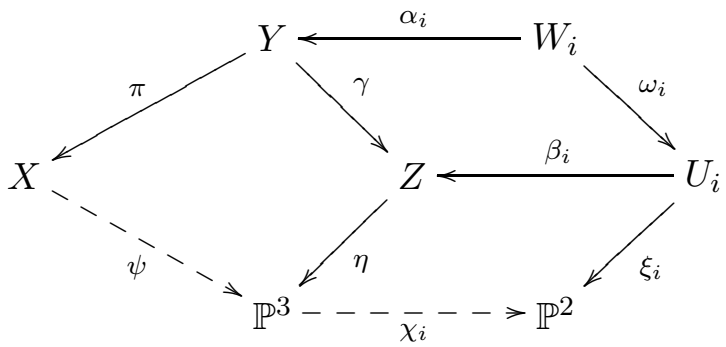

where $\pi$ is the weighted blow up of the point $O$ with weights $(1,1,1)$, the morphism $\gamma$ is the birational morphism that contracts 15 smooth rational curves $C_{1}, \ldots, C_{15}$ to 15 isolated ordinary double points $P_{1}, \ldots, P_{15}$ of the variety $Z$ respectively, $\eta$ is a double cover branched over the surface $R \subset \mathbb{P}^{3}$ of degree 6 that is given by the equation

$$
f_{3}(x, y, z, t)^{2}-4 f_{1}(x, y, z, t) f_{5}(x, y, z, t)=0 \subset \mathbb{P}^{3} \cong \operatorname{Proj}(\mathbb{C}[x, y, z, t])
$$

and has 15 isolated ordinary double points $\eta\left(P_{1}\right), \ldots, \eta\left(P_{15}\right), \alpha_{i}$ is a blow up of $C_{i}, \beta_{i}$ is a blow up of the point $P_{i}, w_{i}$ is a birational morphism, $\chi_{i}$ is a projection from $\eta\left(P_{i}\right)$, and $\xi_{i}$ is an elliptic fibration. Moreover, the points $\eta\left(P_{1}\right), \ldots, \eta\left(P_{15}\right)$ are given by the equations $f_{3}=f_{1}=f_{5}=0$.

Remark 4.1. It follows from [6] that a general fiber of $\chi_{i} \circ \psi$ is $\operatorname{Bir}(X)$-invariant.

In the rest of the section we prove the following result. 
Proposition 4.2. There is a commutative diagram

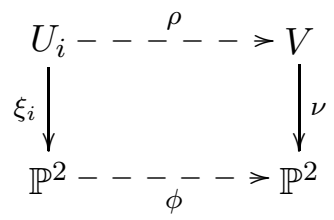

for some $i \in\{1, \ldots, 15\}$, where $\phi$ is a birational map.

Let $\mathcal{B}_{i}$ be the proper transform of the linear system $\mathcal{M}$ on the variety $W_{i}$. Then in order to prove the existence of the commutative diagram 4.3 it is enough to show that $\mathcal{B}_{i}$ lies in the fibers of the elliptic fibration $\xi_{i} \circ \omega_{i}$, which is implied by the equivalence $\mathcal{B}_{i} \sim_{\mathbb{Q}}-k K_{W_{i}}$.

Lemma 4.4. Suppose that $\mathbb{C S}\left(X, \frac{1}{k} \mathcal{M}\right)$ contains a smooth point of $X$. Then the commutative diagram 4.3 exists for some $i \in\{1, \ldots, 15\}$.

Proof. Suppose that $\mathbb{C S}\left(X, \frac{1}{k} \mathcal{M}\right)$ contains a smooth point $P$ of $X$. Let $S$ be a sufficiently general surface in $\left|-K_{X}\right|$ that passes through $P$. In the case when $P \notin \cup_{i=1}^{15} \pi\left(C_{i}\right)$, the surface $S$ does not contain irreducible components of the effective cycle $D_{1} \cdot D_{2}$, where $D_{1}$ and $D_{2}$ are general surfaces of the linear system $\mathcal{M}$. Therefore, in the latter case we have

$$
\operatorname{mult}_{P}\left(D_{1} \cdot D_{2}\right) \leqslant D_{1} \cdot D_{2} \cdot S=-k^{2} K_{X}^{3}=\frac{5}{2} k^{2},
$$

which contradicts Lemma 1.10 in [5]. Thus, the point $P$ is contained in $\pi\left(C_{i}\right)$ for some $i$.

We use arguments of [6]. Put $C=\pi\left(C_{i}\right)$ and $\left.\mathcal{M}\right|_{S}=\mathcal{L}+\operatorname{mult}_{C}(\mathcal{M}) C$, where $\mathcal{L}$ is a linear system on the surface $S$ without fixed components. Then the log pair

$$
\left(S, \frac{1}{k} \mathcal{L}+\frac{\operatorname{mult}_{C}(\mathcal{M})}{k} C\right)
$$

is not $\log$ terminal in $P$ by Theorem 7.5 in [10]. Therefore, we have

$$
\operatorname{mult}_{P}\left(L_{1} \cdot L_{2}\right) \geqslant 4\left(1-\frac{\operatorname{mult}_{C}(\mathcal{M})}{k}\right) k^{2}
$$

by Theorem 3.1 in [5], where $L_{1}$ and $L_{2}$ are general curves in $\mathcal{L}$. On the other hand, the equality

$$
L_{1} \cdot L_{2}=\frac{5}{2} k^{2}-\operatorname{mult}_{C}(\mathcal{M}) k-\frac{3}{2} \operatorname{mult}_{C}^{2}(\mathcal{M})
$$

holds on the surface $S$, because $C^{2}=-3 / 2$. Hence, we have

$$
\frac{5}{2} k^{2}-\operatorname{mult}_{C}(\mathcal{M}) k-\frac{3}{2} \operatorname{mult}_{C}^{2}(\mathcal{M}) \geqslant 4\left(1-\frac{\operatorname{mult}_{C}(\mathcal{M})}{k}\right) k^{2}
$$

which implies mult $C(\mathcal{M})=k$. Thus, the curve $\pi\left(C_{i}\right)$ is contained in $\mathbb{C S}\left(X, \frac{1}{k} \mathcal{M}\right)$, and the equivalence $\mathcal{B}_{i} \sim_{\mathbb{Q}}-k K_{W_{i}}$ follows from Theorem 2.2. which concludes the proof.

We may assume that $\mathbb{C} S\left(X, \frac{1}{k} \mathcal{M}\right)$ does not contain smooth points of $X$.

Lemma 4.5. Let $C$ be a curve on $X$ such that $C \cap \operatorname{Sing}(X)=\varnothing$. Then $C \notin \mathbb{C S}\left(X, \frac{1}{k} \mathcal{M}\right)$.

Proof. Suppose that the set $\mathbb{C S}\left(X, \frac{1}{k} \mathcal{M}\right)$ contains the curve $C$. Then $\operatorname{mult}_{C}(\mathcal{M})=k$. Let $H$ be a very ample divisor on $X$. Then $H \sim \mathbb{Q}-\lambda K_{X}$ holds for a natural number $\lambda$. Thus, we have

$$
\frac{5 \lambda k^{2}}{2}=-\lambda k^{2} K_{X}^{3}=H \cdot S_{1} \cdot S_{2} \geqslant \operatorname{mult}_{C}^{2}(\mathcal{M}) H \cdot C \geqslant-\lambda k^{2} K_{X} \cdot C,
$$

where $S_{1}$ and $S_{2}$ are general surfaces in $\mathcal{M}$. Therefore, we have the following possibilities:

- the equality $-K_{X} \cdot C=1$ holds, and the curve $C$ is smooth and rational;

- the equality $-K_{X} \cdot C=2$ holds, and the curve $C$ is smooth and rational;

- the equality $-K_{X} \cdot C=2$ holds, and the arithmetic genus of the curve $C$ is 1 . 
Let $\sigma: \check{X} \rightarrow X$ be the blow up of the ideal sheaf of the curve $C$, and $G$ be the exceptional divisor of the birational morphism $\sigma$. Then the variety $\check{X}$ is smooth in the neighborhood of the divisor $G$ whenever the curve $C$ is smooth. Moreover, in the case when the curve $C$ has an ordinary double point the singularities of $\check{X}$ in the neighborhood of the divisor $G$ consist of a single isolated ordinary double point. In the case when the curve $C$ has a cuspidal singularity the singularities of the variety $\check{X}$ in the neighborhood of $G$ consist of an isolated double point such that $\check{X}$ in the neighborhood of this point is locally isomorphic to the hypersurface

$$
x_{1}^{2}+x_{2}^{2}+x_{3}^{2}+x_{4}^{3}=0 \subset \mathbb{C} \cong \operatorname{Spec}\left(\mathbb{C}\left[x_{0}, x_{1}, x_{2}, x_{3}\right]\right) .
$$

Let $\breve{S}_{1}$ and $\check{S}_{2}$ be the proper transforms of the surfaces $S_{1}$ and $S_{2}$ on the variety $\check{X}$ respectively.

Suppose that $-K_{X} \cdot C=1$. Then the curve $C$ is cut in the set-theoretic sense by the surfaces of the linear system $\left|-2 K_{X}\right|$ that pass through $C$. Moreover, the scheme-theoretic intersection of two general surfaces of the linear system $\left|-2 K_{X}\right|$ passing through $C$ is reduced in a general point of $C$. Thus, the divisor $\sigma^{*}\left(-2 K_{X}\right)-G$ is nef and big (see Lemma 5.2.5 in [6]), but

$$
\left(\sigma^{*}\left(-2 K_{X}\right)-G\right) \cdot \check{S}_{1} \cdot \check{S}_{2}=0
$$

which is impossible by Corollary 2.7

Suppose that $-K_{X} \cdot C=2$, and $C$ is smooth and rational. Then $\sigma^{*}\left(-2 K_{X}\right)-G$ is nef, because the curve $C$ is cut in the set-theoretic sense by the surfaces of the linear system $\left|-2 K_{X}\right|$ that pass through the curve $C$, but the scheme-theoretic intersection of two general surfaces of the linear system $\left|-2 K_{X}\right|$ passing through $C$ is reduced in a general point of $C$. We have

$$
0>-3 k^{2}=\left(\sigma^{*}\left(-2 K_{X}\right)-G\right) \cdot \check{S}_{1} \cdot \check{S}_{2} \geqslant 0 .
$$

Hence, the arithmetic genus of the curve $C$ is 1 and $-K_{X} \cdot C=2$. The curve $C$ is a set-theoretic intersection of the surfaces in $\left|-4 K_{X}\right|$ that pass through $C$. Moreover, the scheme-theoretic intersection of two general surfaces of the linear system $\left|-4 K_{X}\right|$ passing through $C$ is reduced in a general point $C$. Hence, the divisor $\sigma^{*}\left(-4 K_{X}\right)-G$ is nef and big, but

$$
\left(\sigma^{*}\left(-4 K_{X}\right)-G\right) \cdot \check{S}_{1} \cdot \check{S}_{2}=0
$$

which contradicts Corollary 2.7

It follows from Theorem 2.2 that $O \in \mathbb{C} S\left(X, \frac{1}{k} \mathcal{M}\right)$. Let $\mathcal{D}$ be the proper transform of $\mathcal{M}$ on the variety $Y$. Then Theorem 2.2 implies that $\mathcal{D} \sim_{\mathbb{Q}}-k K_{Y}$. Thus, the commutative diagram4.3 exists in the case when $C_{i} \in \mathbb{C} \mathbb{S}\left(Y, \frac{1}{k} \mathcal{D}\right)$. Therefore, we may assume that

$$
\mathbb{C S}\left(Y, \frac{1}{k} \mathcal{D}\right) \cap\left\{C_{1}, \ldots, C_{15}\right\}=\varnothing .
$$

Lemma 4.6. The set $\mathbb{C S}\left(Y, \frac{1}{k} \mathcal{D}\right)$ does not contain smooth points of the variety $Y$.

Proof. The set $\mathbb{C S}\left(X, \frac{1}{k} \mathcal{M}\right)$ does not contain smooth points of $X$. Therefore, to conclude the proof it is enough to show that the set $\mathbb{C S}\left(Y, \frac{1}{k} \mathcal{D}\right)$ does not contain points of the exceptional divisor of the morphism $\pi$, which is implied by Lemma 2.3

Put $\mathcal{H}=\gamma(\mathcal{D})$. Then $\mathcal{H} \sim_{\mathbb{Q}}-k K_{Z}$, the singularities of the log pair $\left(Z, \frac{1}{k} \mathcal{H}\right)$ are canonical, and the claim of Lemma 2.1 implies that the set $\mathbb{C} S\left(Z, \frac{1}{k} \mathcal{H}\right)$ is not empty.

Lemma 4.7. The set $\mathbb{C S}\left(Z, \frac{1}{k} \mathcal{H}\right)$ does not contain points of the variety $Z$.

Proof. It follows from Lemma 4.6 that smooth points of the variety $Z$ are not contained in the set $\mathbb{C S}\left(Z, \frac{1}{k} \mathcal{H}\right)$. The condition $P_{i} \in \mathbb{C S}\left(Z, \frac{1}{k} \mathcal{H}\right)$ implies that the set $\mathbb{C} \mathbb{S}\left(Y, \frac{1}{k} \mathcal{D}\right)$ contains either the curve $C_{i}$, or a point on the curve $C_{i}$, which is impossible.

Thus, there is a curve $\Gamma$ on $Z$ that is contained in the set $\mathbb{C} S\left(Z, \frac{1}{k} \mathcal{H}\right)$, and $\operatorname{mult}_{\Gamma}(\mathcal{H})=k$.

Lemma 4.8. The equality $-K_{Z} \cdot \Gamma=1$ holds. 
Proof. Let $H$ be a general divisor of the linear system $\left|-K_{Z}\right|$. Then

$$
2 k^{2}=H \cdot D_{1} \cdot D_{2} \geqslant \operatorname{mult}_{\Gamma}\left(D_{1} \cdot D_{2}\right) H \cdot \Gamma \geqslant-k^{2} K_{Z} \cdot \Gamma,
$$

where $D_{1}$ and $D_{2}$ are sufficiently general surfaces of the linear system $\mathcal{H}$. Therefore, the inequality $-K_{Z} \cdot \Gamma \leqslant 2$ holds. Moreover, the equality $-K_{Z} \cdot \Gamma=2$ implies that the support of the effective cycle $D_{1} \cdot D_{2}$ coincides with the curve $\Gamma$, which contradicts Lemma 2.5

The curve $\eta(\Gamma)$ is a line in $\mathbb{P}^{3}$, and $\left.\eta\right|_{\Gamma}: \Gamma \rightarrow \eta(\Gamma)$ is an isomorphism. However, the arguments used in the proof of Lemma 4.8 easily imply that $\mathbb{C S}\left(Z, \frac{1}{k} \mathcal{H}\right)=\{\Gamma\}$ and $\operatorname{mult}_{\Gamma}\left(D_{1} \cdot D_{2}\right)<2 k^{2}$, where $D_{1}$ and $D_{2}$ are general surfaces in $\mathcal{H}$.

Lemma 4.9. The line $\eta(\Gamma)$ is contained in the ramification surface $R$ of the double cover $\eta$.

Proof. Suppose that the line $\eta(\Gamma)$ is not contained in the surface $R$. Let $S$ be a general surface of the linear system $\left|-K_{Z}\right|$ that passes through the curve $\Gamma$. Then

$$
\left.\mathcal{H}\right|_{S}=\operatorname{mult}_{\Gamma}(\mathcal{H}) \Gamma+\operatorname{mult}_{\Omega}(\mathcal{H}) \Omega+\mathcal{L},
$$

where $\mathcal{L}$ is a linear system on the surface $S$ that does not have fixed components, and $\Omega$ is a smooth rational curve on the variety $Z$ such that $\eta(\Omega)=\eta(\Gamma)$, but $\Omega \neq \Gamma$. We have

$$
\operatorname{Sing}(Z) \cap \Gamma=\left\{P_{i_{1}}, \ldots, P_{i_{r}}\right\} \subsetneq\left\{P_{1}, \ldots, P_{15}\right\}=\operatorname{Sing}(Z),
$$

but $P_{i_{j}}$ is an ordinary double point of the surface $S$. The equalities $\Gamma^{2}=\Omega^{2}=-2+r / 2$ hold on the surface $S$, but $r \leqslant 3$. Hence, the inequality $\Omega^{2}<0$ holds on the surface $S$, and

$$
\left(k-\operatorname{mult}_{\Omega}(\mathcal{H})\right) \Omega^{2}=\left(\operatorname{mult}_{\Gamma}(\mathcal{H})-k\right) \Gamma \cdot \Omega+L \cdot \Omega=L \cdot \Omega \geqslant 0,
$$

where $L$ is a general curve in the linear system $\mathcal{L}$. Therefore, the inequality $\operatorname{mult}_{\Omega}(\mathcal{H}) \geqslant k$ holds, which implies that $\Omega \in \mathbb{C} S\left(Z, \frac{1}{k} \mathcal{H}\right)$, which is impossible.

Let $H$ be a general hyperplane in $\mathbb{P}^{3}$ passing through the line $\eta(\Gamma)$. Then the curve

$$
\Delta=H \cap R=\eta(\Gamma) \cup \Upsilon
$$

is reduced and $\eta(\Gamma) \not \subset \operatorname{Supp}(\Upsilon)$, where $\Upsilon$ is a plane curve of degree 5. Moreover, the reducible curve $\Delta$ is singular in every singular point $\eta\left(P_{i}\right)$ of the surface $R$ that lies on the line $\eta(\Gamma)$, but the set $\eta(\Gamma) \cap \Upsilon$ contains at most 5 points. On the other hand, we have

$$
\operatorname{Sing}(\Delta) \cap \eta(\Gamma)=\Upsilon \cap \eta(\Gamma),
$$

which implies that $|\operatorname{Sing}(Z) \cap \Gamma| \leqslant 5$. Moreover, the surface $R$ is given by the equation

$$
f_{3}(x, y, z, t)^{2}=4 f_{1}(x, y, z, t) f_{5}(x, y, z, t) \subset \mathbb{P}^{3} \cong \operatorname{Proj}(\mathbb{C}[x, y, z, t]),
$$

and singular points of $R$ are given by the equations $f_{1}=f_{3}=f_{5}=0$. We may assume that the equations $f_{1}=f_{3}=0$ and $f_{1}=f_{5}=0$ defines irreducible curves in $\mathbb{P}^{3}$, which implies that at most 3 points of the subset $\operatorname{Sing}(R) \subset \mathbb{P}^{3}$ can lie on a single line. Therefore, the Bertini theorem implies that $\eta(\Gamma) \cap \Upsilon$ contains different points $O_{1}$ and $O_{2}$ that are not contained in $\operatorname{Sing}(R)$.

Remark 4.10. The hyperplane $H$ tangents the surface $R$ in the points $O_{1}$ and $O_{2}$.

Let $L_{j}$ be a general line on the plane $H$ that passes through the point $O_{j}, \tilde{O}_{j}$ be a proper transform of the point $O_{j}$ on the variety $Z$, and $\tilde{L}_{j}$ be the proper transform of the line $L_{j}$ on the variety $Z$. Then $L_{j}$ tangents the surface $R$ in the point $O_{j}$, and the curve $\tilde{L}_{j}$ is irreducible and singular in the point $\tilde{O}_{j}$, but $-K_{Z} \cdot \tilde{L}_{j}=2$. Let $\tilde{H}$ be the proper transform of $H$ on the variety $Z$, and $D$ be a general surface of the linear system $\mathcal{H}$. Then

$$
\left.D\right|_{\tilde{H}}=\operatorname{mult}_{\Gamma}(\mathcal{H}) \Gamma+\Psi
$$

where $\Psi$ is an effective divisor on $\tilde{H}$ such that $\Gamma \not \subset \operatorname{Supp}(\Psi)$. Let $\Lambda_{j}=(D \backslash \Gamma) \cap \tilde{L}_{j}$. Then $2 k=D \cdot \tilde{L}_{j} \geqslant \operatorname{mult}_{\tilde{O}_{j}}\left(\tilde{L}_{j}\right) \operatorname{mult}_{\Gamma}(D)+\sum_{P \in \Lambda_{j}} \operatorname{mult}_{P}(D) \cdot \operatorname{mult}_{P}\left(\tilde{L}_{j}\right) \geqslant 2 k+\sum_{P \in \Lambda_{j}} \operatorname{mult}_{P}(D) \cdot \operatorname{mult}_{P}\left(\tilde{L}_{j}\right)$, 
which implies that $D \cap \tilde{L}_{j} \subset \Gamma$. On the other hand, when we vary the lines $L_{1}$ and $L_{2}$ on the plane $H$ the curves $\tilde{L}_{1}$ and $\tilde{L}_{2}$ span two different pencils on the surface $\tilde{H}$, whose base locus consist of the points $\tilde{O}_{1}$ and $\tilde{O}_{1}$ respectively. Hence, we have

$$
\operatorname{Supp}(D) \cap \operatorname{Supp}(\tilde{H})=\operatorname{Supp}(\Gamma),
$$

where $\tilde{H}$ is a general divisor in $\left|-K_{Z}\right|$ that passes through the curve $\Gamma$, and $D$ is a general divisor in $\mathcal{H}$, which contradicts Lemma 2.8 The claim of Proposition 4.2 is proved ${ }^{4}$.

\section{Case $n=4$, hypersurface of Degree 6 in $\mathbb{P}(1,1,1,2,2)$.}

We use the notations and assumptions of Section 3 . Let $n=4$. Then $X$ is a sufficiently general hypersurface in $\mathbb{P}(1,1,1,2,2)$ of degree 6 , the equality $-K_{X}^{3}=3 / 2$ holds, and the singularities of the hypersurface $X$ consist of points $P_{1}, P_{2}, P_{3}$ that are quotient singularities of types $\frac{1}{2}(1,1,1)$.

Let $\psi: X \rightarrow \mathbb{P}^{2}$ be the natural projection. Then a general fiber of the rational map $\psi$ is an elliptic curve, and the composition $\psi \circ \eta$ is a morphism, where $\eta: Y \rightarrow X$ is a composition of the weighted blow ups of the singular points $P_{1}, P_{2}$ and $P_{3}$ with weights $(1,1,1)$.

Proposition 5.1. The claim of Theorem 1.10 holds for $n=4$.

Let us prove Proposition [5.1. We must show the existence of the commutative diagram

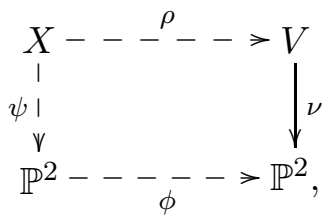

where $\phi \in \operatorname{Bir}\left(\mathbb{P}^{2}\right)$. Let $Z$ be an element of $\mathbb{C S}\left(X, \frac{1}{k} \mathcal{M}\right)$. We have the following possibilities:

- the subvariety $Z$ is a curve that is contained in $X \backslash \operatorname{Sing}(X)$;

- the subvariety $Z$ is a curve that contains a singular point of the hypersurface $X$;

- the subvariety $Z$ is a singular point of the hypersurface $X$.

Suppose that $Z$ is an irreducible curve such that $Z$ does not contain singular points of the hypersurface $X$. Then the equality $-K_{X} \cdot Z=1$ holds by Lemma 2.4, which implies that the curve $Z$ is smooth. Let $\gamma: W \rightarrow X$ be the blow up of the curve $Z$, and $G$ be the exceptional divisor of the morphism $\gamma$. Then the divisor $\gamma^{*}\left(-4 K_{X}\right)-G$ is nef, which implies that

$$
\left(\gamma^{*}\left(-4 K_{X}\right)-G\right) \cdot \bar{S}_{1} \cdot \bar{S}_{2} \geqslant 0
$$

where $\bar{S}_{1}$ and $\bar{S}_{2}$ are proper transforms on the variety $W$ of sufficiently general surfaces of the linear system $\mathcal{M}$. We have $0 \leqslant\left(\gamma^{*}\left(-4 K_{X}\right)-G\right) \cdot \bar{S}_{1} \cdot \bar{S}_{2}=-k^{2}$, which is a contradiction.

Suppose that $Z$ is an irreducible curve that passes through some singular point of the hypersurface $X$. Then the inequality $-K_{X} \cdot Z \leqslant 1$ holds by Lemma 2.4. The curve $C$ is contracted by the rational map $\psi$ to a point, and either $-K_{X} \cdot Z=1 / 2$, or $-K_{X} \cdot Z=1$.

Let $F$ be a sufficiently general surface of the linear system $\left|-K_{X}\right|$ that passes through the curve $Z$. Then the surface $F$ is smooth outside the points $P_{1}, P_{2}$ and $P_{3}$, which are isolated ordinary double points of $F$. Let $\tilde{Z}$ be a fiber of $\psi$ over the point $\psi(Z)$. Then the generality of the hypersurface $X$ implies that the curve $Z$ is an irreducible component of $\tilde{Z}$.

Suppose that $\tilde{Z}$ consists of irreducible curves $Z$ and $\bar{Z}$. Then the inequality $\bar{Z}^{2}<0$ holds on the surface $F$, but $\left.\mathcal{M}\right|_{F} \sim_{\mathbb{Q}} k Z+k \bar{Z}$. On the other hand, we have

$$
\left.\mathcal{M}\right|_{F}=\operatorname{mult}_{Z}(\mathcal{M}) Z+\operatorname{mult}_{\bar{Z}}(\mathcal{M}) \bar{Z}+\mathcal{F},
$$

where $\mathcal{F}$ is a linear system on the surface $F$ that does not have fixed components, but

$$
\left(k-\operatorname{mult}_{\bar{Z}}(\mathcal{M})\right) \bar{Z} \sim_{\mathbb{Q}} \mathcal{F}
$$

which implies that $\operatorname{mult}_{\bar{Z}}(\mathcal{M})=k$ and $\operatorname{Supp}\left(S_{1} \cdot S_{2}\right) \subset Z \cup \bar{Z}$, which contradicts Lemma 2.5 .

\footnotetext{
${ }^{4}$ It is easy to see that the given proof implies the claim of Proposition 4.2 under the weaker assumption that the hypersurface $X$ is quasismooth, and the projection $\psi: X \rightarrow-\mathbb{P}^{3}$ contracts 15 different curves.
} 
Suppose that the fiber $\tilde{Z}$ consists of irreducible curves $Z, \hat{Z}$ and $\check{Z}$. Then

$$
-K_{X} \cdot \hat{Z}=-K_{X} \cdot \check{Z}=-K_{X} \cdot Z=\frac{1}{2},
$$

but the intersection form of the curves $\hat{Z}$ and $\check{Z}$ on the surface $F$ is negatively defined, which implies that the support of $S_{1} \cdot S_{2}$ is contained in $Z \cup \hat{Z} \cup \check{Z}$, where $S_{1}$ and $S_{2}$ are general surfaces of the linear system $\mathcal{M}$, which is impossible by Lemma 2.5.

Hence, we have $\mathbb{C} \mathbb{S}\left(X, \frac{1}{k} \mathcal{M}\right) \subseteq\left\{P_{1}, P_{2}, P_{3}\right\}$.

Let $\pi: U \rightarrow X$ be a composition of the weighted blow ups with weights $(1,1,1)$ of the singular points of the hypersurface $X$ that are contained in the set $\mathbb{C S}\left(X, \frac{1}{k} \mathcal{M}\right)$, and $\mathcal{D}$ be the proper transform of the linear system $\mathcal{M}$ on the variety $U$. Then it follows from Theorem 2.2 that the equivalence $\mathcal{D} \sim_{\mathbb{Q}}-k K_{U}$ holds, but the divisor $-K_{U}$ is nef.

Suppose that $\mathbb{C} S\left(U, \frac{1}{k} \mathcal{D}\right)$ contains a subvariety $\Delta \subset U$. Then $\Delta$ is contained in some exceptional divisor of $\pi$. Let $G$ be an exceptional divisor of $\pi$ that contains $\Delta$. Then $\Delta$ is a line on the surface $G \cong \mathbb{P}^{2}$ by Lemma 2.3. The linear system $\left|\pi^{*}\left(-2 K_{X}\right)-G\right|$ does not have base points, and the divisor $\pi^{*}\left(-2 K_{X}\right)-G$ is nef and big. It follows from Lemma 0.3.3 in [9] that there is a proper Zariski closed subset $\Lambda \subset U$ such that $\Lambda$ contains all curves on $U$ having trivial intersections with the divisor $\pi^{*}\left(-2 K_{X}\right)-G$. We have

$$
2 k^{2}=\left(\pi^{*}\left(-2 K_{X}\right)-G\right) \cdot \tilde{S}_{1} \cdot \tilde{S}_{2} \geqslant \operatorname{mult}_{\Delta}^{2}(\mathcal{D})\left(\pi^{*}\left(-2 K_{X}\right)-G\right) \cdot \Delta=2 k^{2},
$$

where $\tilde{S}_{1}$ and $\tilde{S}_{2}$ are the proper transforms of $S_{1}$ and $S_{2}$ on $U$ respectively. Thus, the support of the effective cycle $\tilde{S}_{1} \cdot \tilde{S}_{2}$ is contained in $\Lambda \cup \Delta$, which is impossible by Lemma 2.5.

Hence, the set $\mathbb{C S}\left(U, \frac{1}{k} \mathcal{D}\right)$ is empty, and $-K_{U}^{3}=0$ by Lemma 2.1. Then $\pi=\eta$, which implies that $\mathcal{D}$ lies in the fibers of $\psi \circ \pi$. Therefore, the commutative diagram 5.2 exists.

\section{Case $n=6$, hypersurface of Degree 8 IN $\mathbb{P}(1,1,1,2,4)$.}

We use the notations and assumptions of Section 3. Let $n=6$. Then $X$ is a sufficiently general hypersurface in $\mathbb{P}(1,1,1,2,4)$ of degree 8 , the equality $-K_{X}^{3}=1$ holds, and the singularities of the hypersurface $X$ consists of points $P_{1}$ and $P_{2}$ that are singularities of type $\frac{1}{2}(1,1,1)$.

There is a commutative diagram

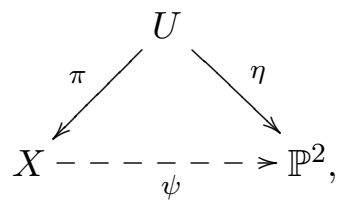

where $\psi$ is the natural projection, $\pi$ is a composition of the weighted blow ups of the singular points $P_{1}$ and $P_{2}$ with weights $(1,1,1)$, and $\eta$ is an elliptic fibration.

Proposition 6.2. The claim of Theorem 1.10 holds for $n=6$.

Let us prove Proposition 6.2 It follows from Theorem 3.5 that the set $\mathbb{C} \mathbb{S}\left(X, \frac{1}{k} \mathcal{M}\right)$ does not contain smooth points of the hypersurface $X$. Therefore, the set $\mathbb{C} S\left(X, \frac{1}{k} \mathcal{M}\right)$ contains a singular point of the hypersurface $X$ by Corollary 3.6 and Theorem 2.2 .

Remark 6.3. Suppose that the set $\mathbb{C S}\left(X, \frac{1}{k} \mathcal{M}\right)$ contains both points $P_{1}$ and $P_{2}$. Then it easily follows from Theorem 2.2 that the claim of the Theorem 1.10 holds for the hypersurface $X$.

We may assume that $P_{1} \in \mathbb{C} \mathbb{S}\left(X, \frac{1}{k} \mathcal{M}\right)$ and $P_{2} \notin \mathbb{C S}\left(X, \frac{1}{k} \mathcal{M}\right)$.

Lemma 6.4. The set $\mathbb{C} \mathbb{S}\left(X, \frac{1}{k} \mathcal{M}\right)$ does not contain curves.

Proof. Suppose that $\mathbb{C S}\left(X, \frac{1}{k} \mathcal{M}\right)$ contains a curve $C$. Then $-K_{X} \cdot C=1 / 2$ by Lemma 2.4

Let $\check{C}$ be the proper transform of the curve $C$ on the variety $U$. Then $-K_{U} \cdot \check{C}=0$, which implies that the curve $\check{C}$ is a component of a fiber of $\eta$. Therefore, the curve $C$ is contracted by the rational map $\psi: X \rightarrow \mathbb{P}^{2}$ to a point. In particular, the curve $C$ is smooth and rational.

Let $S$ be a general surface of the linear system $\left|-K_{X}\right|$ that contains the curve $C$. Then the surface $S$ is smooth outside the points $P_{1}$ and $P_{2}$, which are isolated ordinary double points on 
the surface $S$. Let $F$ be a fiber of the rational map $\psi$ over the point $\psi(C)$. Then $F$ consists of two irreducible components such that the curve $C$ is one of them. Let $Z$ be the component of the curve $F$ that is different from the curve $C$. Then $Z^{2}<0$ on the surface $S$, but

$$
\left.\mathcal{M}\right|_{S}=\operatorname{mult}_{C}(\mathcal{M}) C+\operatorname{mult}_{Z}(\mathcal{M}) Z+\mathcal{L},
$$

where $\mathcal{L}$ is a linear system having no fixed components. We have $\left(k-\operatorname{mult}_{Z}(\mathcal{M})\right) Z \sim_{\mathbb{Q}} \mathcal{L}$, which implies that $\operatorname{mult}_{Z}(\mathcal{M})=k$. The latter is impossible by Lemmas 2.8 and 2.10

Thus, the set $\mathbb{C S}\left(X, \frac{1}{k} \mathcal{M}\right)$ consists of the point $P_{1}$. Let $\zeta: Y \rightarrow X$ be the weighted blow up of the point $P_{1}$ with weights $(1,1,1)$, and $\mathcal{D}$ be the proper transform of the linear system $\mathcal{M}$ on the variety $Y$. Then $\mathcal{D} \sim_{\mathbb{Q}}-k K_{Y}$ by Theorem 2.2 and $\mathbb{C S}\left(Y, \frac{1}{k} \mathcal{D}\right) \neq \varnothing$ by Lemma 2.1.

Let $T$ be a subvariety of $Y$ that is contained in $\mathbb{C S}\left(Y, \frac{1}{k} \mathcal{D}\right)$. Then $\zeta(T) \in \mathbb{C} \mathbb{S}\left(X, \frac{1}{k} \mathcal{M}\right)$, which is impossible by Lemmas 2.3 and 2.4 .

\section{Case $n=7$, hypersurface of Degree 8 In $\mathbb{P}(1,1,2,2,3)$.}

We use the notations and assumptions of Section 3 Let $n=7$. Then $X$ is a sufficiently general hypersurface in $\mathbb{P}(1,1,2,2,3)$ of degree 8 , whose singularities consist of the point $Q$ that is a quotient singularity of type $\frac{1}{3}(1,1,2)$, and the points $P_{1}, P_{2}, P_{3}$ and $P_{4}$ that are quotient singularities of type $\frac{1}{2}(1,1,1)$. There is a commutative diagram

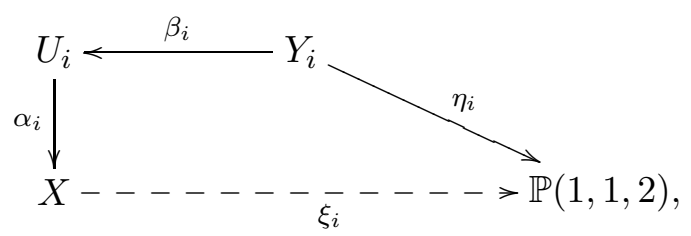

where $\xi_{i}$ is a projection, $\alpha_{i}$ is the weighted blow up of $P_{i}$ with weights $(1,1,1), \beta_{i}$ is the weighted blow up of the proper transform of $Q$ on the variety $U_{i}$ with weights $(1,1,2)$, and $\eta_{i}$ is an elliptic fibration. There is a commutative diagram

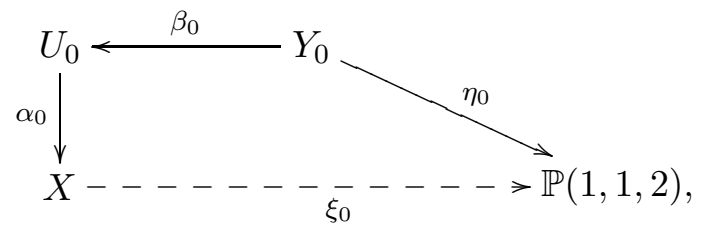

where $\xi_{0}$ is a projection, $\alpha_{0}$ is the weighted blow up of $Q$ with weights $(1,1,2), \beta_{0}$ is the blow up with weights $(1,1,1)$ of the singular point of $U_{0}$ that dominates $Q$, and $\eta_{0}$ is an elliptic fibration.

Proposition 7.1. There is a commutative diagram

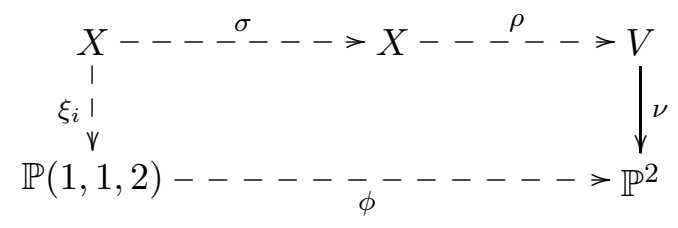

for some $i \in\{0,1,2,3,4\}$, where $\sigma$ and $\phi$ are birational maps.

Let us prove Proposition [7.1. The set $\mathbb{C S}\left(X, \frac{1}{k} \mathcal{M}\right)$ does not contains smooth points of the hypersurface $X$, and it follows from Lemma 3.14 that $\mathbb{C S}\left(X, \frac{1}{k} \mathcal{M}\right) \subseteq\left\{P_{1}, P_{2}, P_{3}, P_{4}, Q\right\}$.

Lemma 7.3. The set $\mathbb{C S}\left(X, \frac{1}{k} \mathcal{M}\right)$ does not contain two points of the set $\left\{P_{1}, P_{2}, P_{3}, P_{4}\right\}$.

Proof. Suppose that the set $\mathbb{C S}\left(X, \frac{1}{k} \mathcal{M}\right)$ contains the points $P_{1}$ and $P_{2}$. Let $\pi: W \rightarrow X$ be the composition of the weighted blow ups of $P_{1}$ and $P_{2}$ with weights $(1,1,1)$, and $\mathcal{B}$ be the proper transform of the linear system $\mathcal{M}$ on the variety $W$. Then $\mathcal{B} \sim_{\mathbb{Q}}-k K_{W}$ by Theorem [2.2] but the base locus of $\left|-K_{W}\right|$ consists of a curve $C$ such that $C^{2}=-1 / 3$ on general surface of the pencil $\left|-K_{W}\right|$, which contradicts Lemma 2.8.

Let $\mathcal{D}_{i}$ be the proper transform of the linear system $\mathcal{M}$ on the variety $U_{i}$. 
Remark 7.4. The set $\mathbb{C S}\left(U_{0}, \frac{1}{k} \mathcal{D}_{0}\right)$ is not empty by Lemma 2.1 if the set $\mathbb{C S}\left(X, \frac{1}{k} \mathcal{M}\right)$ contains the point $Q$. Similarly, the set $\mathbb{C} S\left(U_{i}, \frac{1}{k} \mathcal{D}_{i}\right)$ is not empty, if $\mathbb{C} \mathbb{S}\left(X, \frac{1}{k} \mathcal{M}\right)$ contains the point $P_{i}$.

Lemma 7.5. The set $\mathbb{C S}\left(X, \frac{1}{k} \mathcal{M}\right)$ does not consists of the point $P_{i}$.

Proof. Suppose that $\mathbb{C S}\left(X, \frac{1}{k} \mathcal{M}\right)=\left\{P_{i}\right\}$. Then the set $\mathbb{C} \mathbb{S}\left(U_{i}, \frac{1}{k} \mathcal{D}_{i}\right)$ contains an irreducible subvariety $Z \subset U_{i}$ by Lemma 2.1. Let $E_{i}$ be the exceptional divisor of $\alpha_{i}$. Then $Z$ is a line on the surface $E_{i} \cong \mathbb{P}^{2}$ by Lemma 2.3. which contradicts Lemma 2.4.

Therefore, the condition $P_{i} \in \mathbb{C} \mathbb{S}\left(X, \frac{1}{k} \mathcal{M}\right)$ implies that $\mathbb{C} S\left(X, \frac{1}{k} \mathcal{M}\right)=\left\{P_{i}, Q\right\}$, which easily implies that the proper transform of the linear system $\mathcal{M}$ on the variety $Y_{i}$ lies in the fibers of the elliptic fibration $\eta_{i}$. The latter implies the existence of the commutative diagram 7.2 .

We may assume that the set $\mathbb{C} S\left(X, \frac{1}{k} \mathcal{M}\right)$ consists of the point $Q$.

Let $O$ be a singular point of the variety $U_{0}$ that is contained in the exceptional divisor of the morphism $\alpha_{0}$. Then $O$ is contained in $\mathbb{C S}\left(U_{0}, \frac{1}{k} \mathcal{D}_{0}\right)$ by Lemma 2.3, which implies the existence of the commutative diagram 7.2 by Theorem 2.2. The claim of Proposition 7.1 is proved.

\section{Case $n=8$, hypersurface of Degree 9 IN $\mathbb{P}(1,1,1,3,4)$.}

We use the notations and assumptions of Section 3, Let $n=8$. Then $X$ is a sufficiently general hypersurface in $\mathbb{P}(1,1,1,3,4)$ of degree 9 , the equality $-K_{X}^{3}=3 / 4$ holds, and the singularities of the hypersurface $X$ consist of the singular point $O$ that is a quotient singularity of type $\frac{1}{4}(1,1,3)$.

Proposition 8.1. The claim of Theorem 1.10 holds for $n=8$.

Let us prove Proposition 8.1 . The hypersurface $X$ can be given by the equation

$$
w^{2} z+f_{5}(x, y, z, t) w+f_{9}(x, y, z, t)=0 \subset \mathbb{P}(1,1,1,3,5) \cong \operatorname{Proj}(\mathbb{C}[x, y, z, t, w]),
$$

where $\operatorname{wt}(x)=\operatorname{wt}(y)=\operatorname{wt}(z)=1, \operatorname{wt}(t)=3, \operatorname{wt}(w)=4$, and $f_{i}$ is a quasihomogeneous polynomial of degree $i$. The point $O$ is given by $x=y=z=t=0$. There is a commutative diagram

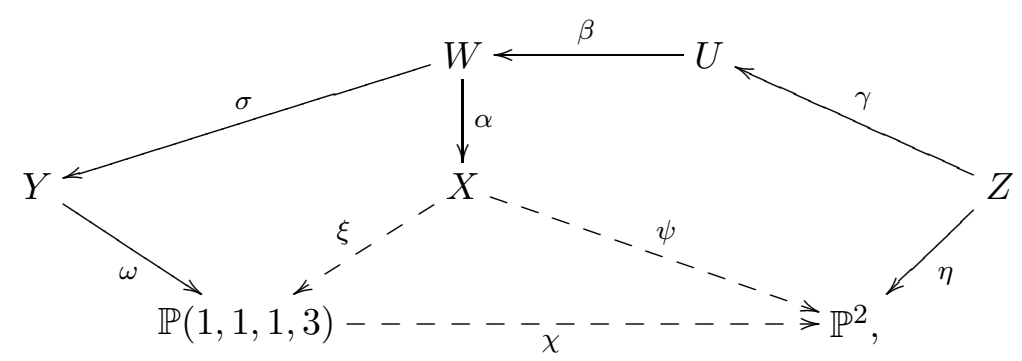

where rational maps $\xi, \psi$ and $\chi$ are the natural projections, $\alpha$ is the weighted blow up of the singular point $O$ with weights $(1,1,3), \beta$ is the weighted blow up with weights $(1,1,2)$ of the singular point of the variety $W$ that is a quotient singularity of type $\frac{1}{3}(1,1,2), \gamma$ is the weighted blow up with weights $(1,1,1)$ of the singular point of the variety $U$ that is a quotient singularity of type $\frac{1}{2}(1,1,1), \eta$ is an elliptic fibration, $\sigma$ is a birational morphism that contracts 15 smooth rational curves to 15 isolated ordinary double points $P_{1}, \ldots, P_{15}$ of $Y$ respectively, $\omega$ is a double cover branched over the surface $R \subset \mathbb{P}(1,1,1,3)$ that is given by the equation

$$
f_{5}(x, y, z, t)^{2}-4 z f_{9}(x, y, z, t)=0 \subset \mathbb{P}(1,1,1,3) \cong \operatorname{Proj}(\mathbb{C}[x, y, z, t])
$$

and has 15 isolated ordinary double points $\omega\left(P_{1}\right), \ldots, \omega\left(P_{15}\right)$ given by $z=f_{5}=f_{9}=0$.

Let $G$ be the exceptional divisor of the morphism $\alpha, F$ be the exceptional divisor of the morphism $\beta, P$ be the singular point of $W, Q$ be the singular point of $U, \mathcal{D}$ be the proper transform of $\mathcal{M}$ on $W, \mathcal{H}$ be the proper transform of $\mathcal{M}$ on the variety $U, \mathcal{P}$ be the proper transform of $\mathcal{M}$ on the variety $Z$, and $\mathcal{B}$ be the proper transform of $\mathcal{M}$ on the variety $Y$.

Remark 8.2. The divisors $-K_{W}$ and $-K_{U}$ are nef and big, and $\omega \circ \sigma(G)$ is given by $z=0$.

Lemma 8.3. The set $\mathbb{C} S\left(X, \frac{1}{k} \mathcal{M}\right)$ does not contain curves. 
Proof. Let $L$ be a curve in $\mathbb{C S}\left(X, \frac{1}{k} \mathcal{M}\right)$. It follows from Theorem 3.12 that there are two different surfaces $D$ and $D^{\prime}$ in the linear system $\left|-K_{X}\right|$ such that the irreducible curve $L$ is a component of the cycle $D \cdot D^{\prime}$. The cycle $D \cdot D^{\prime}$ is reduced and contains at most two components.

Let $\mathcal{P}$ be the pencil in $\left|-K_{X}\right|$ that is generated by the surfaces $D$ and $D^{\prime}$. Then we may assume that $D$ is a sufficiently general surface of the pencil $\mathcal{P}$. Applying Lemma 2.8 together with the proof of Lemma 2.4 to the linear system $\mathcal{M}$ and the pencil $\mathcal{P}$, we immediately obtain a contradiction in the case when the equality $-K_{X} \cdot L=3 / 4$ holds. Therefore, we may assume that either $-K_{X} \cdot L=1 / 4$ or $-K_{X} \cdot L=1 / 2$. We consider only the case $-K_{X} \cdot L=1 / 4$, because the case $-K_{X} \cdot L=1 / 2$ is simpler and very similar.

Let $S_{W}$ be he proper transform on $W$ of the surface given by $z=0$, and $L_{W}$ be the proper transform on $W$ of the curve $L$. Then $S_{W}$ must contain $L_{W}$, because

$$
S_{W} \sim_{\mathbb{Q}} \alpha^{*}\left(-K_{X}\right)-\frac{5}{4} G,
$$

but $G \cdot L_{W} \geqslant 1 / 3$. Thus, either the curve $L_{W}$ is contracted by $\sigma$ or the curve $\omega\left(L_{Y}\right)$ is a ruling of the cone $\mathbb{P}(1,1,1,3)$ contained in the surface $\omega \circ \sigma(G)$, where $L_{Y}$ is the image of $L_{W}$ by $\sigma$.

Suppose that the curve $L_{W}$ is not contracted by $\sigma$. Then, the curve $\omega\left(L_{Y}\right)$ is not contained in the surface $R$, which implies that $\omega\left(L_{Y}\right)$ contains at most one singular point of $R$ that is different from the point $\omega \circ \sigma(P)$. Moreover, the curve $\omega\left(L_{Y}\right)$ must contain a singular point of the surface $R$ different from $\omega \circ \sigma(P)$ because $-K_{X} \cdot L=3 / 4$ otherwise. Thus, we may assume that the curve $\omega\left(L_{Y}\right)$ contains the point $\omega\left(P_{1}\right)$.

Let $D_{Y}$ and $D_{Y}^{\prime}$ be the proper transforms on $Y$ of the surfaces $D$ and $D^{\prime}$ respectively. Then the point $P_{1}$ is an isolated ordinary double point of the surface $D_{Y}$. Thus, we see that the proper transform of the curve $L^{\prime}$ on the threefold $W$ is contracted to the point $P_{1}$ by $\sigma$ and

$$
D_{Y} \cdot D_{Y}^{\prime}=L_{Y}+\bar{L}_{Y}
$$

where $\bar{L}_{Y}$ is a ruling of the cone $G \cong \mathbb{P}(1,1,3)$. In particular, we have $-K_{X} \cdot L^{\prime}=1 / 4$, which contradicts the equality $-K_{X} \cdot L=1 / 4$. Hence, the curve $L_{W}$ is contracted by $\sigma$.

Let $L_{W}^{\prime}$ be the proper transform on $W$ of the curve $L^{\prime}$, and $L_{Y}^{\prime}=\sigma\left(L_{W}^{\prime}\right)$. Then $\omega\left(L_{Y}^{\prime}\right)$ is a ruling of the cone $\mathbb{P}(1,1,1,3)$ that is contained in the surface $\omega \circ \sigma(G)$. The curve $\omega\left(L_{Y}^{\prime}\right)$ is not contained in the surface $R$, which implies that $\omega\left(L_{Y}^{\prime}\right)$ contains at most one singular point of the surface $R$ different from the point $\omega \circ \sigma(P)$. The curve $\omega\left(L_{Y}^{\prime}\right)$ must contain a singular point of the surface $R$ different from $\omega \circ \sigma(P)$ because $-K_{X} \cdot L^{\prime} \neq 3 / 4$. Thus, we may assume that the curve $\omega\left(L_{Y}^{\prime}\right)$ contains the point $\omega\left(P_{1}\right)$.

The point $P_{1}$ is an isolated ordinary double point of $D_{Y}$ and $\sigma\left(L_{W}\right)=P_{1}$. Hence, we have

$$
D_{Y} \cdot D_{Y}^{\prime}=L_{Y}^{\prime}+\bar{L}_{Y}^{\prime}
$$

where $\bar{L}_{Y}^{\prime}$ is a ruling of $G \cong \mathbb{P}(1,1,3)$. The intersection $L_{W} \cap L_{W}^{\prime}$ consists of a point $O^{\prime}$ such that $O^{\prime} \notin G$. Hence, the intersection $L \cap L^{\prime}$ contains the point $\alpha\left(O^{\prime}\right)$ that is different from $O$.

The surface $D$ is smooth at the point $\alpha\left(O^{\prime}\right)$, but $\left(L+L^{\prime}\right) \cdot L^{\prime}=1 / 2$ on the surface $D$, which implies that $L^{\prime} \cdot L^{\prime}<0$ on the surface $D$. Therefore, we have

$$
\left.\mathcal{M}\right|_{D}=m_{1} L+m_{2} L^{\prime}+\mathcal{L} \equiv k L+k L^{\prime},
$$

where $\mathcal{L}$ is a linear system on $D$ that does not have fixed components, and $m_{1}$ and $m_{2}$ are natural numbers such that $m_{1} \geqslant k$. In particular, we have

$$
0 \leqslant\left(m_{1}-k\right) L^{\prime} \cdot L+\mathcal{L} \cdot L^{\prime}=\left(k-m_{2}\right) L^{\prime} \cdot L^{\prime},
$$

which implies that $m_{2}=m_{1}=k$ and $\left.\mathcal{M}\right|_{D}=k L+k L^{\prime}$, which is impossible by Lemma 2.8,

It follows from Theorem 3.5 that $\mathbb{C S}\left(X, \frac{1}{k} \mathcal{M}\right)=\{O\}$. Hence, the claim of Theorem 2.2 implies that $\mathcal{D} \sim_{\mathbb{Q}}-k K_{W}$, but $P \in \mathbb{C} \mathbb{S}\left(W, \frac{1}{k} \mathcal{D}\right)$ by Lemmas 2.1 and 2.3

Lemma 8.4. Suppose that the set $\mathbb{C} S\left(W, \frac{1}{k} \mathcal{D}\right)$ does not contain subvarieties of $W$ that are different from the point $P$. Then the claim of Theorem 1.10 holds for the hypersurface $X$.

Proof. The equivalence $\mathcal{H} \sim_{\mathbb{Q}}-k K_{U}$ holds by Theorem 2.2. Hence, the set $\mathbb{C} \mathbb{S}\left(U, \frac{1}{k} \mathcal{H}\right)$ contains the point $Q$ by Lemmas 2.1 and 2.3. We see that $\mathcal{P}$ lies in the fibers of $\eta$ by Theorem 2.2. 
We may assume that the set $\mathbb{C S}\left(W, \frac{1}{k} \mathcal{D}\right)$ contains a subvariety $Z$ that is different from the point $P$. Then $Z$ is a curve in $G$ by Lemma 2.3. which is impossible by Corollary 2.9.

9. Case $n=9$, hypersurface of Degree 9 IN $\mathbb{P}(1,1,2,3,3)$.

We use the notations and assumptions of Section 3. Let $n=9$. Then $X$ is a sufficiently general hypersurface in $\mathbb{P}(1,1,2,3,3)$ of degree 9 , the equality $-K_{X}^{3}=1 / 2$ holds, and the singularities of the hypersurface $X$ consist of the point $O$ that is a quotient singularity of type $\frac{1}{2}(1,1,1)$, and the points $P_{1}, P_{2}$ and $P_{3}$ that are quotient singularities of type $\frac{1}{3}(1,1,2)$.

There is a commutative diagram

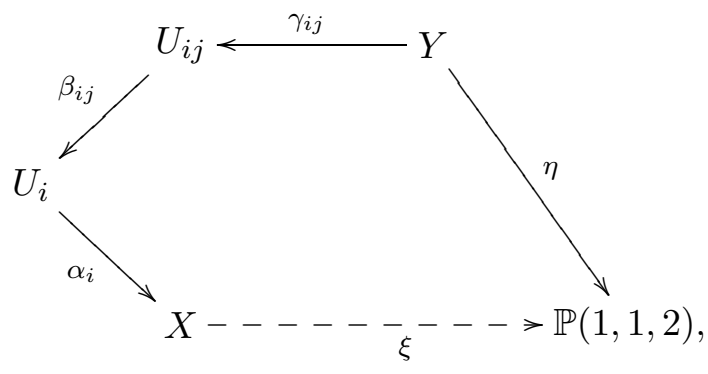

where $\xi_{i}$ is a projection, $\alpha_{i}$ is the blow up of $P_{i}$ with weights $(1,1,2), \beta_{i j}$ is the weighted blow up with weights $(1,1,2)$ of the proper transform of $P_{j}$ on the variety $U_{i}, \gamma_{i j}$ is the weighted blow up with weights $(1,1,2)$ of the proper transform of $P_{k}$ on the $U_{i j}$, and $\eta$ is an elliptic fibration, where $i \neq j$ and $k \notin\{i, j\}$.

Remark 9.1. The divisors $-K_{U_{i}}$ and $-K_{U_{i j}}$ are nef and big.

There is a commutative diagram

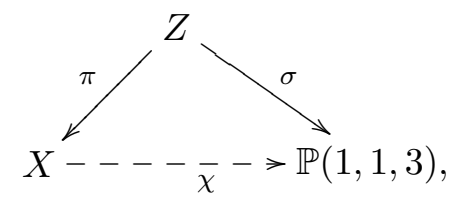

where $\chi$ is a projection, $\pi$ is the blow up of $Q$ with weights $(1,1,1)$, and $\sigma$ is an elliptic fibration.

Proposition 9.2. Either there is a commutative diagram

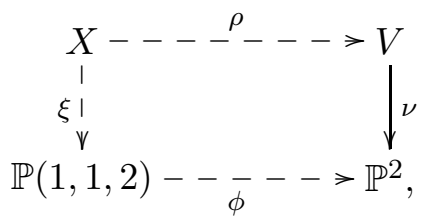

or there is a commutative diagram

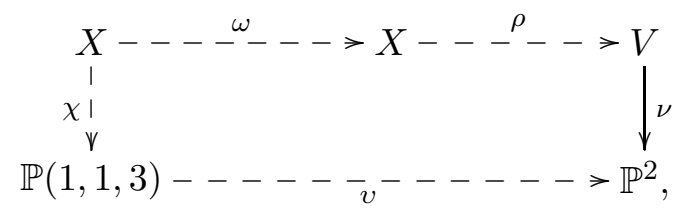

where $\phi, \omega$ and $v$ are birational maps.

Let us prove Proposition 9.2, which implies the claim of Theorem 1.10 for $n=9$.

Lemma 9.5. Suppose that $Q \in \mathbb{C} S\left(X, \frac{1}{k} \mathcal{M}\right)$. Then there is a commutative diagram 9.4.

Proof. Let $\mathcal{B}$ be the proper transform of $\mathcal{M}$ on $Z$. Then $\mathcal{B} \sim_{\mathbb{Q}}-k K_{X}$ by Theorem 2.2] which implies the equality $S \cdot C=0$, where $S$ is a general surface of the linear system $\mathcal{M}$, and $C$ is a general fiber of the morphism $\sigma$. Thus, the commutative diagram 9.4 exists.

Lemma 9.6. The commutative diagram 9.3 exists whenever $\mathbb{C S}\left(X, \frac{1}{k} \mathcal{M}\right)=\left\{P_{1}, P_{2}, P_{3}\right\}$.

Proof. See the proof of Lemma 9.5 . 
We may assume that $\mathbb{C S}\left(X, \frac{1}{k} \mathcal{M}\right) \subseteq\left\{P_{2}, P_{3}\right\}$ by Theorem 3.5 and Lemma 3.14.

Lemma 9.7. The set $\mathbb{C S}\left(X, \frac{1}{k} \mathcal{M}\right)$ contains the point $P_{2}$.

Proof. Suppose that the set $\mathbb{C S}\left(X, \frac{1}{k} \mathcal{M}\right)$ consists of the point $P_{1}$. Let $\mathcal{B}$ be the proper transform of the linear system $\mathcal{M}$ on the variety $U_{1}$. Then $\mathcal{B} \sim_{\mathbb{Q}}-k K_{U_{1}}$ by Theorem [2.2, and Lemma 2.1 implies that the set $\mathbb{C} \mathbb{S}\left(U_{1}, \frac{1}{k} \mathcal{B}\right)$ is not empty.

Let $Z$ be an element of the set $\mathbb{C S}\left(U_{1}, \frac{1}{k} \mathcal{B}\right)$, and $G$ be the exceptional divisor of the birational morphism $\alpha_{1}$. Then it follows from Lemmas 2.3 and 2.4 that $Z$ is a singular point of $G$, which is a quotient singularity of type $\frac{1}{2}(1,1,1)$ on the variety $U_{1}$.

Let $\delta: W \rightarrow U_{1}$ be the weighted blow up of the point $Z$ with weights $(1,1,1), \mathcal{D}$ be the proper transform of the linear system $\mathcal{M}$ on the variety $W$, and $F$ be a general surface of the linear system $\left|-K_{W}\right|$. Then the inequality $\Delta^{2}=-1 / 2$ holds on $F$, but $\left.\mathcal{D}\right|_{F} \sim_{\mathbb{Q}} k \Delta$ by Theorem 2.2 which is impossible by Lemmas 2.10 and 2.8 .

Now we can apply the arguments of the proof of Lemma 9.7 to get a contradiction.

\section{Case $n=10$, hypersurface of Degree 10 In $\mathbb{P}(1,1,1,3,5)$.}

We use the notations and assumptions of Section [3, Let $n=10$. Then $X$ is a sufficiently general hypersurface in $\mathbb{P}(1,1,1,3,5)$ of degree 10 , the equality $-K_{X}^{3}=2 / 3$ holds, the singularities of the hypersurface $X$ consist of the point $O$ that is a quotient singularity of type $\frac{1}{3}(1,1,2)$.

It should be pointed out that $X$ is birationally superrigid.

Proposition 10.1. The claim of Theorem 1.10 holds for $n=10$.

There is a commutative diagram

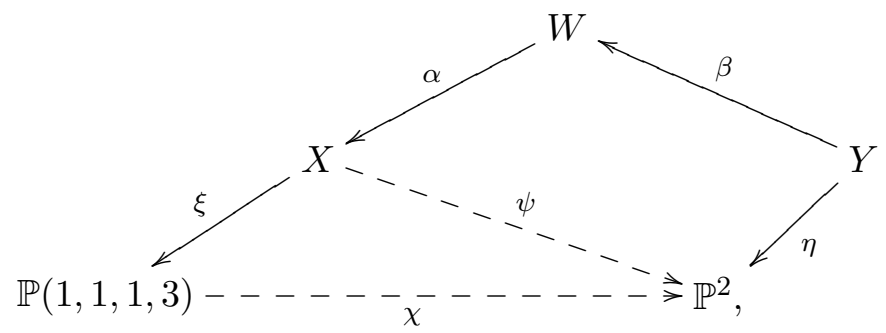

where $\xi, \psi$ and $\chi$ are projections, $\alpha$ is the weighted blow up of $O$ with weights $(1,1,1), \beta$ is the weighted blow up with weights $(1,1,1)$ of the singular point of $W$, and $\eta$ is an elliptic fibration.

In the rest of the section we prove Proposition 10.1. Let $Q$ be the unique singular point of the variety $W, \mathcal{D}$ be the proper transforms of the linear system $\mathcal{M}$ on the variety $W$, and $\mathcal{H}$ be the proper transforms of the linear system $\mathcal{M}$ on the variety $Y$.

Lemma 10.2. The set $\mathbb{C S}\left(X, \frac{1}{k} \mathcal{M}\right)$ does not contains curves.

Proof. Suppose that $\mathbb{C S}\left(X, \frac{1}{k} \mathcal{M}\right)$ contains a curve $C$. Then $-K_{X} \cdot C=1 / 3$ by Lemma 2.4 , which implies that the curve $C$ is contracted by the rational map $\psi$ to a point.

There is a curve $Z$ on the variety $X$ such that $Z \neq C$, but $\xi(Z)=\xi(C)$. Let $S$ be a general surface of the linear system $\left|-K_{X}\right|$ that passes through $C$ and $Z$. Then $Z^{2}<0$ on the normal surface $S$, but $\left.\mathcal{M}\right|_{S} \sim_{\mathbb{Q}} k C+k Z$, which is impossible by Lemmas 2.8 and 2.10.

The set $\mathbb{C S}\left(X, \frac{1}{k} \mathcal{M}\right)$ consists of the point $O$ by Theorem 3.5 the set $\mathbb{C S}\left(W, \frac{1}{k} \mathcal{D}\right)$ contains the point $Q$ by Lemmas 2.1 and 2.3 , but $\mathcal{H} \sim \mathbb{Q}-k K_{Y}$ by Theorem 2.2. which implies that $\mathcal{H}$ is contained in the fibers of $\eta$. The claim of Proposition 10.1 is proved.

\section{Case $n=12$, hypersurface of Degree 10 in $\mathbb{P}(1,1,2,3,4)$.}

We use the notations and assumptions of Section [3 Let $n=12$. Then $X$ is a general hypersurface in $\mathbb{P}(1,1,2,3,4)$ of degree 10 , whose singularities consist of the points $P_{1}$ and $P_{2}$ that 
are singularities of type $\frac{1}{2}(1,1,1)$, the point $P_{3}$ that is a singularity of type $\frac{1}{3}(1,1,2)$, and the point $P_{4}$ that is a singularity of type $\frac{1}{4}(1,1,3)$. There is a commutative diagram

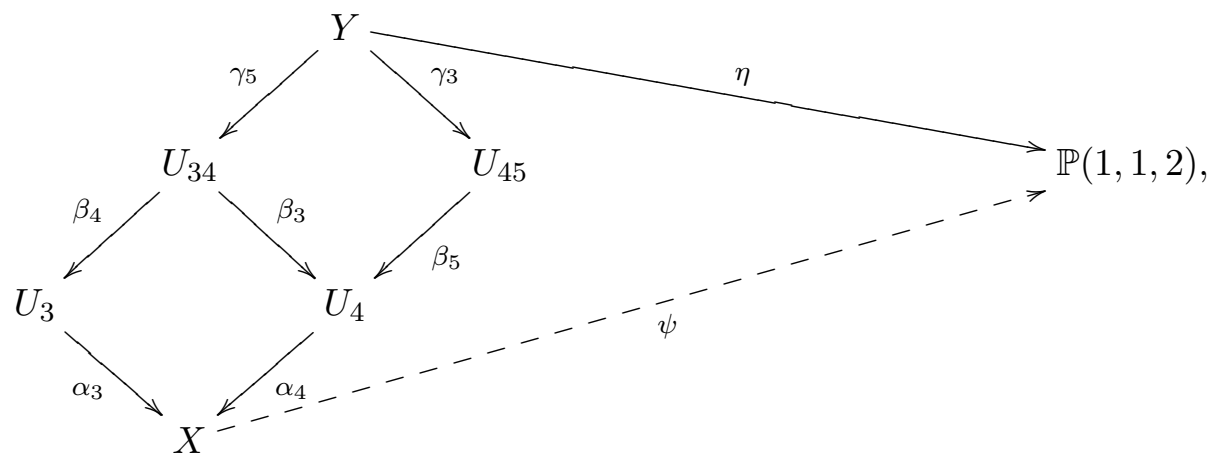

where $\psi$ is a projection, $\alpha_{3}$ is the weighted blow up of $P_{3}$ with weights $(1,1,2), \alpha_{4}$ is the weighted blow up of $P_{4}$ with weights $(1,1,3), \beta_{4}$ is the weighted blow up with weights $(1,1,3)$ of the proper transform of the point $P_{4}$ on $U_{3}, \beta_{3}$ is the weighted blow up with weights $(1,1,2)$ of the proper transform of the point $P_{3}$ on $U_{4}, \beta_{5}$ is the weighted blow up with weights $(1,1,2)$ of the singular point of $U_{4}$ that is contained in the exceptional divisor of $\alpha_{4}, \gamma_{3}$ is the weighted blow up with weights $(1,1,2)$ of the proper transform of the point $P_{3}$ on the variety $U_{45}, \gamma_{5}$ is the weighted blow up with weights $(1,1,2)$ of the singular point of $U_{34}$ that is contained in the exceptional divisor of the morphism $\beta_{4}$, and $\eta$ is an elliptic fibration.

Proposition 11.1. The claim of Theorem 1.10 holds for $n=12$.

In the rest of this sectiop we prove Proposition 11.1

Remark 11.2. The divisors $-K_{U_{3}},-K_{U_{4}},-K_{U_{34}}$ and $-K_{U_{45}}$ are nef and big.

It follows from Theorem 3.5 and Lemma 3.14 that $\mathbb{C S}\left(X, \frac{1}{k} \mathcal{M}\right) \subseteq\left\{P_{1}, P_{2}, P_{3}, P_{4}\right\}$.

Lemma 11.3. The set $\mathbb{C S}\left(X, \frac{1}{k} \mathcal{M}\right)$ does not contain $P_{1}$ and $P_{2}$.

Proof. Suppose that $P_{1} \in \mathbb{C} \mathbb{S}\left(X, \frac{1}{k} \mathcal{M}\right)$. Let $\pi: W \rightarrow X$ be the weighted blow up of the singular point $P_{1}$ with weights $(1,1,1), \mathcal{B}$ be the proper transform of $\mathcal{M}$ on $W, S$ be a general surface of the pencil $\left|-K_{W}\right|$, and $Z$ be the base curve of the pencil $\left|-K_{W}\right|$. Then $Z^{2}=-1 / 24$ on the surface $S$, but $\left.\mathcal{B}\right|_{S} \sim_{\mathbb{Q}} k Z$, which contradicts Lemmas 2.10 and 2.8

Let $\mathcal{D}_{3}, \mathcal{D}_{4}, \mathcal{D}_{34}$ and $\mathcal{D}_{45}$ be the proper transforms of $\mathcal{M}$ on $U_{3}, U_{4}, U_{34}$ and $U_{45}$ respectively, then it follows from Lemma 2.1 that $\mathbb{C S}\left(U_{\mu}, \frac{1}{k} \mathcal{D}_{\mu}\right) \neq \varnothing$ in the case when $\mathcal{D}_{\mu} \sim_{\mathbb{Q}}-k K_{U_{\mu}}$.

Lemma 11.4. Suppose that the set $\mathbb{C S}\left(X, \frac{1}{k} \mathcal{M}\right)$ contains the point $P_{4}$. Let $\bar{P}_{3}$ be the proper transform of the point $P_{3}$ on the variety $U_{4}$, and $\bar{P}_{5}$ be the singular point of the variety $U_{4}$ that dominates the point $P_{4}$. Then $\mathcal{D}_{4} \sim_{\mathbb{Q}}-k K_{U_{4}}$ and $\mathbb{C S}\left(U_{4}, \frac{1}{k} \mathcal{D}_{4}\right) \subseteq\left\{\bar{P}_{3}, \bar{P}_{5}\right\}$.

Proof. Suppose that $\mathbb{C S}\left(U_{4}, \frac{1}{k} \mathcal{D}_{4}\right) \nsubseteq\left\{\bar{P}_{3}, \bar{P}_{5}\right\}$. Let $C$ be an element of $\mathbb{C} \mathbb{S}\left(U_{4}, \frac{1}{k} \mathcal{D}_{4}\right)$ different from the points $\bar{P}_{3}$ and $\bar{P}_{5}$. Then $\alpha_{4}(C)=P_{4}$, which contradicts Lemma 2.3 and Corollary 2.9 ,

Lemma 11.5. Suppose that the set $\mathbb{C S}\left(X, \frac{1}{k} \mathcal{M}\right)$ contains the point $P_{3}$. Let $\bar{P}_{4}$ be the proper transform of the point $P_{4}$ on the variety $U_{3}$. Then $\mathcal{D}_{3} \sim_{\mathbb{Q}}-k K_{U_{3}}$ and $\mathbb{C S}\left(U_{3}, \frac{1}{k} \mathcal{D}_{3}\right)=\left\{\bar{P}_{4}\right\}$.

Proof. See the proof of Lemma 9.7

It follows from Theorem 2.2 that either $\mathcal{D}_{34} \sim_{\mathbb{Q}}-k K_{U_{34}}$ or $\mathcal{D}_{45} \sim_{\mathbb{Q}}-k K_{U_{45}}$. Let $\mathcal{D}$ be the proper transform of the linear system $\mathcal{M}$ on the variety $Y$.

Lemma 11.6. Suppose that $\mathcal{D}_{34} \sim_{\mathbb{Q}}-k K_{U_{34}}$. Then $\mathcal{D} \sim_{\mathbb{Q}}-k K_{Y}$.

Proof. Let $F$ be the exceptional divisor of the morphism $\beta_{3}, G$ be the exceptional divisor of the morphism $\beta_{4}, \check{P}_{5}$ be the singular point of the surface $G$, and $\check{P}_{6}$ be the singular point of the surface $F$. Then $\mathcal{D} \sim_{\mathbb{Q}}-k K_{Y}$ by Theorem 2.2 if the set $\mathbb{C S}\left(U_{34}, \frac{1}{k} \mathcal{D}_{34}\right)$ contains the point $\check{P}_{5}$.

Suppose that $\check{P}_{5} \notin \mathbb{C S}\left(U_{34}, \frac{1}{k} \mathcal{D}_{34}\right)$. Then $\check{P}_{6} \in \mathbb{C S}\left(U_{34}, \frac{1}{k} \mathcal{D}_{34}\right)$ by Lemmas 2.1 and 2.3 . 
Let $\pi: W \rightarrow U_{34}$ be the weighted blow up of the point $\check{P}_{6}$ with weights $(1,1,1), \mathcal{B}$ be the proper transform of the linear system $\mathcal{M}$ on the variety $W$, and $S$ be a general surface of the linear system $\left|-K_{W}\right|$. Then the base locus of $\left|-K_{W}\right|$ consists of the irreducible curve $\Delta$ such that $\left.\mathcal{B}\right|_{S} \sim_{\mathbb{Q}} k \Delta$, but $\Delta^{2}<0$ holds on $S$, which contradicts Lemmas [2.10] and 2.8.

The proof of Lemma 11.6 implies that $\mathcal{D} \sim_{\mathbb{Q}}-k K_{Y}$ whenever $\mathcal{D}_{45} \sim_{\mathbb{Q}}-k K_{U_{45}}$, but it follows from the equivalence $\mathcal{D} \sim_{\mathbb{Q}}-k K_{Y}$ that the linear system $\mathcal{D}$ lies in the fibers of the elliptic fibrations $\eta$. Hence, the claim of Proposition 11.1 is proved.

\section{Case $n=13$, hypersurface of Degree 11 in $\mathbb{P}(1,1,2,3,5)$.}

We use the notations and assumptions of Section 3. Let $n=13$. Then $X$ is a sufficiently general hypersurface in $\mathbb{P}(1,1,2,3,5)$ of degree 11 , the singularities of the hypersurface $X$ consist of the point $P_{1}$ that is a quotient singularity of type $\frac{1}{2}(1,1,1)$, the point $P_{2}$ that is a singularity of type $\frac{1}{3}(1,1,2)$, the point $P_{3}$ that is a singularity of type $\frac{1}{5}(1,2,3)$, and $-K_{X}^{3}=11 / 30$.

Proposition 12.1. The claim of Theorem 1.10 holds for $n=13$.

Let us prove Proposition 12.1. There is a commutative diagram

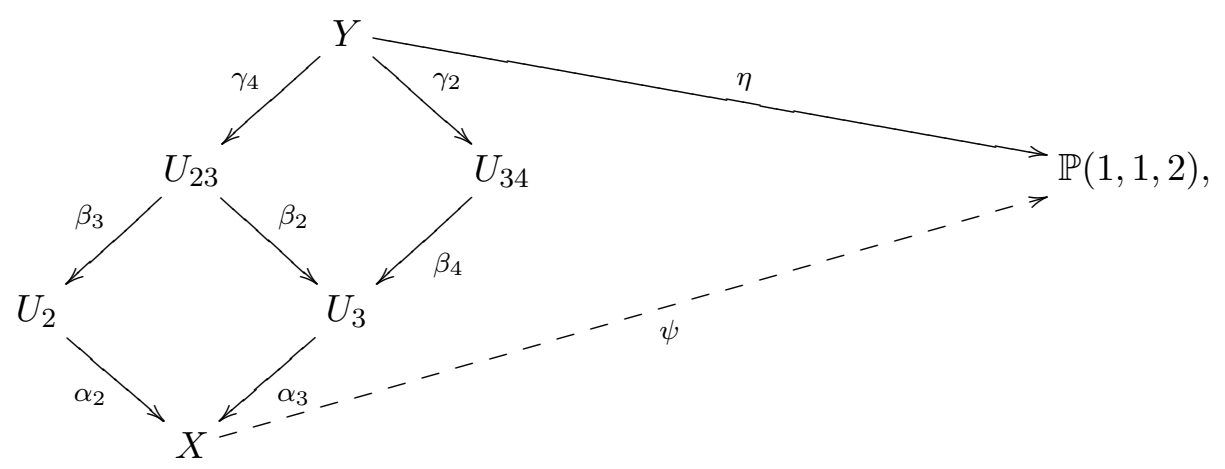

where $\psi$ is a projection, $\alpha_{2}$ is the weighted blow up of $P_{2}$ with weights $(1,1,2), \alpha_{3}$ is the weighted blow up of $P_{3}$ with weights $(1,2,3), \beta_{3}$ is the weighted blow up with weights $(1,2,3)$ of the proper transform of the point $P_{3}$ on the variety $U_{2}, \beta_{2}$ is the weighted blow up with weights $(1,1,2)$ of the proper transform of $P_{2}$ on the variety $U_{3}, \beta_{4}$ is the weighted blow up with weights $(1,1,2)$ of the singular point of the variety $U_{3}$ that is the quotient singularity of type $\frac{1}{3}(1,1,2)$ contained in the exceptional divisor of $\alpha_{3}, \gamma_{2}$ is the weighted blow up with weights $(1,1,2)$ of the proper transform of $P_{2}$ on the variety $U_{34}, \gamma_{4}$ is the weighted blow up with weights $(1,1,2)$ of the singular point of the variety $U_{23}$ that is the quotient singularity of type $\frac{1}{3}(1,1,2)$ contained in the exceptional divisor of the morphism $\beta_{3}$, and $\eta$ is an elliptic fibration.

It follows from Theorem 3.5. Lemma 3.14 and Proposition 3.7 that $\mathbb{C S}\left(X, \frac{1}{k} \mathcal{M}\right) \subseteq\left\{P_{2}, P_{3}\right\}$.

Lemma 12.2. The set $\mathbb{C S}\left(X, \frac{1}{k} \mathcal{M}\right)$ contains the point $P_{3}$.

Proof. Suppose that $P_{3} \notin \mathbb{C S}\left(X, \frac{1}{k} \mathcal{M}\right)$. Then $\mathbb{C} S\left(X, \frac{1}{k} \mathcal{M}\right)=\left\{P_{2}\right\}$. Let $\mathcal{D}_{2}$ be the proper transform of $\mathcal{M}$ on the variety $U_{2}$. Then it follows from Theorem 2.2 that $\mathcal{D}_{2} \sim_{\mathbb{Q}}-k K_{U_{2}}$, but the set $\mathbb{C} \mathbb{S}\left(U_{2}, \frac{1}{k} \mathcal{D}_{2}\right)$ is not empty by Lemma 2.1

Let $E$ be the exceptional divisor of the morphism $\alpha_{2}$. Then $E$ can be identified with a cone over the smooth rational curve in $\mathbb{P}^{3}$ of degree 3 . Let $Z$ be a subvariety of the variety $U_{2}$ that is contained in the set $\mathbb{C S}\left(U_{2}, \frac{1}{k} \mathcal{D}_{2}\right)$. Then it follows from Lemmas 2.3 and 2.4 that $Z$ is the vertex of the cone $E$, which is a quotient singularity of type $\frac{1}{2}(1,1,1)$ on the variety $U_{2}$.

Let $\pi: W \rightarrow U_{2}$ be the blow up of the point $Z$ with weights $(1,1,1)$, and $S$ be a sufficiently general surface of the pencil $\left|-K_{W}\right|$. Then the base locus of the pencil $\left|-K_{W}\right|$ consists of an irreducible curve $\Delta$ such that $\Delta^{2}=-3 / 10$ on the surface $S$, but $\left.\mathcal{B}\right|_{S} \sim_{\mathbb{Q}} k \Delta$, where $\mathcal{B}$ is the proper transform of $\mathcal{M}$ on $W$. We have $\left.\mathcal{B}\right|_{S}=k \Delta$, which is impossible by Lemma 2.8 .

Lemma 12.3. The set $\mathbb{C S}\left(X, \frac{1}{k} \mathcal{M}\right)$ contains the point $P_{2}$. 
Proof. Suppose that $\mathbb{C S}\left(X, \frac{1}{k} \mathcal{M}\right)$ does not contain the point $P_{2}$. Then $\mathbb{C} \mathbb{S}\left(X, \frac{1}{k} \mathcal{M}\right)$ consists of the point $P_{3}$. Let $\mathcal{D}_{3}$ be the proper transform of $\mathcal{M}$ on $U_{3}$. Then $\mathbb{C S}\left(U_{3}, \frac{1}{k} \mathcal{D}_{3}\right) \neq \varnothing$ by Lemma 2.1 .

Let $G$ be the exceptional divisor of the morphism $\alpha_{3}, P_{4}$ be the singular point of $G$ that is a quotient singularity of type $\frac{1}{3}(1,1,2)$ on the variety $U_{3}$, and $P_{5}$ be the singular point of the surface $G$ that is a quotient singularity of type $\frac{1}{2}(1,1,1)$ on $U_{3}$. Then $G \cong \mathbb{P}(1,2,3)$, and it follows from Lemma 2.3 that either $\mathbb{C} S\left(U_{3}, \frac{1}{k} \mathcal{D}_{3}\right)=\left\{P_{4}\right\}$, or $P_{5} \in \mathbb{C} \mathbb{S}\left(U_{3}, \frac{1}{k} \mathcal{D}_{3}\right)$.

Suppose that the set $\mathbb{C} S\left(U_{3}, \frac{1}{k} \mathcal{D}_{3}\right)$ contains the point $P_{5}$. Let $\pi: W \rightarrow U_{3}$ be the weighted blow up of $P_{5}$ with weights $(1,1,1), \mathcal{B}$ be the proper transform of $\mathcal{M}$ on $W, L$ be the curve on the surface $G$ that is contained in $\left|\mathcal{O}_{\mathbb{P}(1,2,3)}(1)\right|, \bar{L}$ be the proper transform of the curve $L$ on the variety $W$, and $S$ be a general surface in $\left|-K_{W}\right|$. Then $\left.\mathcal{B}\right|_{S} \sim_{\mathbb{Q}} k \Delta+k \bar{L}$ by Theorem 2.2 the base locus of $\left|-K_{W}\right|$ consists of the curves $\bar{L}$ and $\Delta$ such that $\alpha \circ \pi(\Delta)$ is the base curve of the pencil $\left|-K_{X}\right|$. The equalities

$$
\Delta^{2}=-5 / 6, \bar{L}^{2}=-4 / 3, \Delta \cdot \bar{L}=1
$$

hold on the surface $S$, which imply that the intersection form of the curves $\Delta$ and $\bar{L}$ on the surface $S$ is negatively defined. The latter is impossible by Lemmas 2.8 and 2.10.

Hence, the set $\mathbb{C} \mathbb{S}\left(U_{3}, \lambda \mathcal{D}_{3}\right)$ consists of the point $P_{4}$. Let $D_{34}$ be the proper transform of the linear system $\mathcal{M}$ on the variety $U_{34}$. Then $\mathbb{C} S\left(U_{34}, \frac{1}{k} \mathcal{D}_{34}\right) \neq \varnothing$ by Lemma 2.1] because the equivalence $D_{34} \sim_{\mathbb{Q}}-k K_{U_{34}}$ holds by Theorem 2.2

Let $E$ be the exceptional divisor of the morphism $\beta_{4}$, and $P_{6}$ be the singular point of the surface $E$. Then $E \cong \mathbb{P}(1,1,3)$, the point $P_{6}$ is a quotient singularity of type $\frac{1}{2}(1,1,1)$ on the variety $U_{34}$, and $P_{6} \in \mathbb{C} \mathbb{S}\left(U_{34}, \frac{1}{k} \mathcal{D}_{34}\right)$ by Lemma 2.3

Let $\zeta: Z \rightarrow U_{34}$ be the weighted blow up of $P_{6}$ with weights $(1,1,1), \mathcal{H}$ be the proper transform of $\mathcal{M}$ on $Z$, and $F$ be a general surface of the pencil $\left|-K_{Z}\right|$. Then the base locus of the pencil $\left|-K_{Z}\right|$ consists of irreducible curves $\check{L}$ and $\breve{\Delta}$ such that the equalities

$$
\check{\Delta}^{2}=-5 / 6, \check{L}^{2}=-3 / 2, \check{\Delta} \cdot \check{L}=1
$$

hold on $F$. Thus, the intersection form of the curves $\check{\Delta}$ and $\check{L}$ on the surface $F$ is negatively defined, but $\left.\mathcal{H}\right|_{F} \sim_{\mathbb{Q}} k \breve{\Delta}+k \breve{L}$ by Theorem 2.2. which contradicts Lemmas 2.8 and 2.10.

Let $\mathcal{D}_{23}$ be the proper transform of $\mathcal{M}$ on $U_{23}$. Then $\mathbb{C} S\left(U_{23}, \frac{1}{k} \mathcal{D}_{23}\right) \neq \varnothing$ by Lemma 2.1.

Remark 12.4. The proof of Lemma 12.2 implies that the set $\mathbb{C} S\left(U_{23}, \frac{1}{k} \mathcal{D}_{23}\right)$ does no contain the singular point of the variety $U_{23}$ that is contained in the exceptional divisor of $\beta_{2}$, but the proof of Lemma 12.3 implies that $\mathbb{C S}\left(U_{23}, \frac{1}{k} \mathcal{D}_{23}\right)$ does not contain the singular point of $U_{23}$ that is a singularity of type $\frac{1}{2}(1,1,1)$ contained in the exceptional divisor of $\beta_{3}$.

Therefore, the set $\mathbb{C S}\left(U_{23}, \frac{1}{k} \mathcal{D}_{23}\right)$ contains the singular point the variety $U_{23}$ that is a singularity of type $\frac{1}{3}(1,1,2)$ contained in the $\beta_{3}$-exceptional divisor, which implies that the proper transform of $\mathcal{M}$ on the variety $Y$ is contained in the fibers of $\eta$ by Theorem 2.2 .

\section{Case $n=15$, hypersurface of Degree 12 in $\mathbb{P}(1,1,2,3,6)$.}

We use the notations and assumptions of Section 3. Let $n=15$. Then $X$ is a sufficiently general hypersurface in $\mathbb{P}(1,1,2,3,6)$ of degree 12 , the equality $-K_{X}^{3}=1 / 3$ holds, and the singularities of the hypersurface $X$ consist of the points $P_{1}$ and $P_{2}$ that are quotient singularities of type $\frac{1}{2}(1,1,1)$, and the points $P_{3}$ and $P_{4}$ that are quotient singularities of type $\frac{1}{3}(1,1,2)$.

There is a commutative diagram

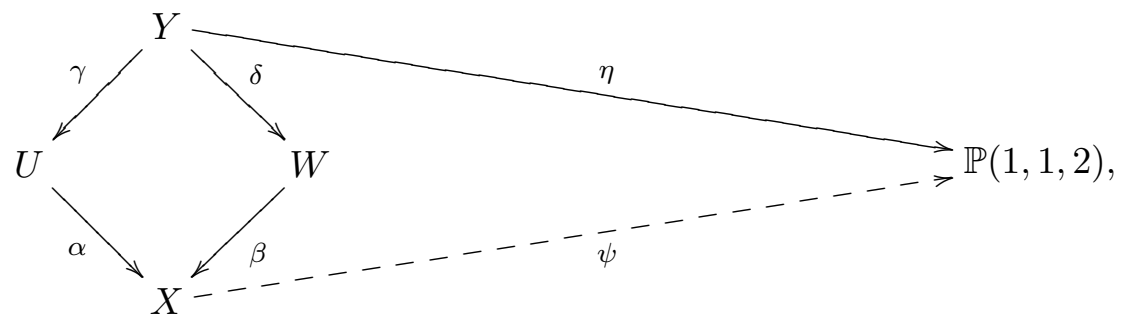


where $\psi$ is a projection, $\alpha$ is the weighted blow up of the point $P_{3}$ with weights $(1,1,2), \beta$ is the weighted blow up of $P_{4}$ with weights $(1,1,2), \gamma$ is the weighted blow up with weights $(1,1,2)$ of the proper transform of $P_{4}$ on the variety $U, \delta$ is the weighted blow up with weights $(1,1,2)$ of the proper transform of the point $P_{3}$ on the variety $W$, and $\eta$ is an elliptic fibration.

Proposition 13.1. The claim of Theorem 1.10 holds for $n=15$.

Proof. We have $\mathbb{C} S\left(X, \frac{1}{k} \mathcal{M}\right) \subseteq\left\{P_{3}, P_{4}\right\}$ by Theorem 3.5. Proposition 3.7 and Lemma 3.14,

Let $\mathcal{H}$ be the proper transform of the linear system $\mathcal{M}$ on the variety $Y$. To conclude the proof we must show that $\mathcal{H}$ lies in the fibers of the morphism $\eta$, which easily follows from the condition $\mathbb{C S}\left(X, \frac{1}{k} \mathcal{M}\right)=\left\{P_{3}, P_{4}\right\}$ by Theorem 2.2 . We may assume that $P_{4} \notin \mathbb{C S}\left(X, \frac{1}{k} \mathcal{M}\right)$.

Let $\mathcal{B}$ be the proper transform of $\mathcal{M}$ on $U$, and $Q$ be the singular point of $U$ that is contained in the exceptional divisor of the morphism $\alpha$. Then $\mathcal{B} \sim_{\mathbb{Q}}-k K_{U}$ by Theorem [2.2 and it follows from Lemmas 2.1 and 2.3 that the set $\mathbb{C S}\left(U, \frac{1}{k} \mathcal{B}\right)$ contains the singular point $Q$.

Let $\zeta: Z \rightarrow U$ be the weighted blow up of the point $Q$ with weights $(1,1,1), \mathcal{D}$ be the proper transform of $\mathcal{M}$ on the threefold $Z$, and $S$ be a general surface in $\left|-K_{Z}\right|$. Then the base locus of the pencil $\left|-K_{Z}\right|$ consists of an irreducible curve $\Delta$ such that $\Delta^{2}<0$ on the surface $S$, which is impossible by Lemmas 2.10 and 2.8, because $\left.\mathcal{D}\right|_{S} \sim_{\mathbb{Q}}-k \Delta$ by Theorem 2.2

\section{Case $n=16$, hypersurface of Degree 12 in $\mathbb{P}(1,1,2,4,5)$.}

We use the notations and assumptions of Section [3 Let $n=16$. Then $X$ is a sufficiently general hypersurface in $\mathbb{P}(1,1,2,4,5)$ of degree 12 , the equality $-K_{X}^{3}=3 / 10$ holds, and the singularities of the hypersurface $X$ consist of the points $P_{1}, P_{2}$ and $P_{3}$ that are quotient singularities of type $\frac{1}{2}(1,1,1)$, and the point $P_{4}$ that is a quotient singularity of type $\frac{1}{5}(1,1,4)$.

Proposition 14.1. The claim of Theorem 1.10 holds for $n=16$.

Let us prove Proposition 14.1. There is a commutative diagram

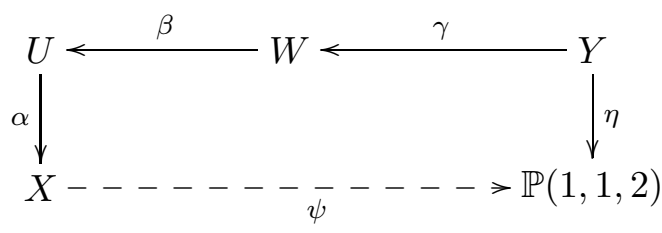

where $\psi$ is the natural projection, $\alpha$ is the weighted blow up of $P_{4}$ with weights $(1,1,4), \beta$ is the weighted blow up with weights $(1,1,3)$ of the singular point of the variety $U$ that is contained in the exceptional divisor of $\alpha, \gamma$ is the weighted blow up with weights $(1,1,2)$ of the singular point of $W$ that is contained in the exceptional divisor of $\beta$, and $\eta$ is an elliptic fibration.

Remark 14.2. The divisors $-K_{U}$ and $-K_{W}$ are nef and big.

It follows from Theorem 3.5. Lemma 3.14 and Proposition 3.7 that $\mathbb{C S}\left(X, \frac{1}{k} \mathcal{M}\right)$ consists of the point $P_{4}$. Let $G$ be the exceptional divisor of the morphism $\alpha, \bar{P}_{5}$ be the singular point of the surface $G$, and $\mathcal{D}$ be the proper transform of $\mathcal{M}$ on the variety $U$. Then $G$ is a cone over a smooth rational curve of degree 4 , and $\bar{P}_{5}$ is a singularity of type $\frac{1}{4}(1,1,3)$ on the variety $U$.

We see that $\mathcal{D} \sim_{\mathbb{Q}}-k K_{U}$ by Theorem 2.2 and $\mathbb{C S}\left(U, \frac{1}{k} \mathcal{D}\right) \neq \varnothing$ by Lemma 2.1

Lemma 14.3. The set $\mathbb{C} \mathbb{S}\left(U, \frac{1}{k} \mathcal{D}\right)$ consists of the point $\bar{P}_{5}$.

Proof. Suppose that the set $\mathbb{C S}\left(U, \frac{1}{k} \mathcal{D}\right)$ contains a subvariety $C$ of the variety $U$ that is different from the point $\bar{P}_{5}$. Then it follows from Lemma 2.3 that $C$ is a ruling of the cone $G$, which is impossible by Corollary 2.9

Let $\mathcal{H}$ be the proper transform of $\mathcal{M}$ on $W$. Then it follows from Theorem 2.2 and Lemmas 2.1 and 2.3 that the set $\mathbb{C S}\left(W, \frac{1}{k} \mathcal{H}\right)$ contains the singular point of the variety $W$ that is contained in the exceptional divisor of $\beta$. Therefore, the proper transform of the linear system $\mathcal{M}$ on the variety $Y$ lies in the fibers of the morphism $\eta$ by Theorem 2.2 
15. Case $n=17$, hypersurface of Degree 12 in $\mathbb{P}(1,1,3,4,4)$.

We use the notations and assumptions of Section 3. Let $n=17$. Then $X$ is a general hypersurface in $\mathbb{P}(1,1,3,4,4)$ of degree 12 , whose singularities consist of the points $P_{1}, P_{2}$ and $P_{3}$ that are singularities of type $\frac{1}{4}(1,1,3)$. There is a commutative diagram

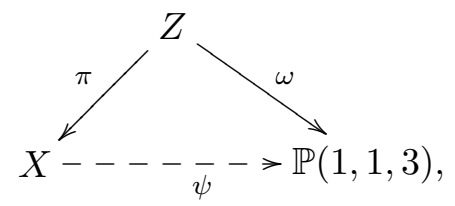

where $\psi$ is a projection, $\pi$ is a composition of the weighted blow ups of $P_{1}, P_{2}$ and $P_{3}$ with weights $(1,1,3)$, and $\omega$ is and elliptic fibration. There is a commutative diagram

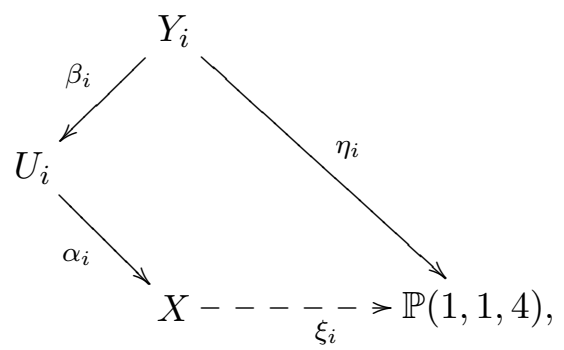

where $\xi_{i}$ is a projection, $\alpha_{i}$ is the blow up of $P_{i}$ with weights $(1,1,3), \beta_{i}$ is the weighted blow up with weights $(1,1,2)$ of the singular point the variety $U_{i}$ that is contained in the exceptional divisor of the morphism $\alpha_{i}$, and $\eta_{i}$ is an elliptic fibration.

Proposition 15.1. Either there is a commutative diagram

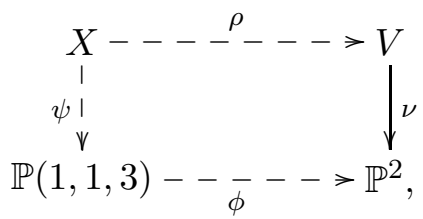

or there is a commutative diagram

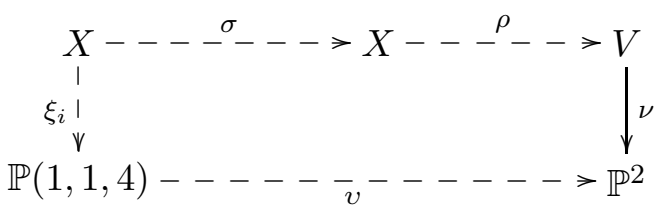

for some $i \in\{1,2,3\}$, where $\phi, \sigma$ and $v$ are birational maps.

Let us prove Proposition 15.1 which implies the claim of Theorem 1.10 for $n=17$.

Remark 15.4. It easily follows from Theorem 2.2 that the commutative diagram 15.2 exists, if the set $\mathbb{C} \mathbb{S}\left(X, \frac{1}{k} \mathcal{M}\right)$ contains the point $P_{1}, P_{2}$ and $P_{3}$.

It follows from Theorem 3.5 and Lemma 3.14 that $\mathbb{C S}\left(X, \frac{1}{k} \mathcal{M}\right) \subseteq\left\{P_{1}, P_{2}, P_{3}\right\}$, but we may assume that the set $\mathbb{C} S\left(X, \frac{1}{k} \mathcal{M}\right)$ contains the point $P_{1}$ and does not contain the point $P_{3}$.

Remark 15.5. The divisor $-K_{U_{1}}$ is nef and big.

Let $\mathcal{D}$ be the proper transform of the linear system $\mathcal{M}$ on the variety $U_{1}, \bar{P}_{2}$ be the proper transform of the point $P_{2}$ on the variety $U_{1}$, and $\bar{P}_{4}$ be the singular point of the variety $U_{1}$ that is contained in the exceptional divisor of $\alpha_{1}$. Then $\mathcal{D} \sim_{\mathbb{Q}}-k K_{U_{1}}$ by Theorem 2.2. and it follows from Lemma 2.1 that the set $\mathbb{C} S\left(U_{1}, \frac{1}{k} \mathcal{D}\right)$ is not empty.

Remark 15.6. It easily follows from Theorem 2.2 that the commutative diagram 15.3 exists in the case when the set $\mathbb{C} S\left(U_{1}, \frac{1}{k} \mathcal{D}\right)$ contains the point $\bar{P}_{4}$. 
Therefore, we may assume that $\mathbb{C S}\left(U_{1}, \frac{1}{k} \mathcal{D}\right)$ does not contain the point $\bar{P}_{4}$. Hence, it follows from the proof of Lemma 9.7 that $\mathbb{C} \mathbb{S}\left(U_{1}, \frac{1}{k} \mathcal{D}\right)$ does not contains subvarieties of $U_{1}$ that are contained in the exceptional divisor of $\alpha_{1}$. Thus, the set $\mathbb{C} \mathbb{S}\left(U_{1}, \frac{1}{k} \mathcal{D}\right)$ contains $\bar{P}_{3}$.

Let $\gamma: W \rightarrow U_{1}$ be the weighted blow up of the point $\bar{P}^{3}$ with weights $(1,1,3), \mathcal{B}$ is the proper transform of $\mathcal{M}$ on the variety $W, S$ be a general surface of in $\left|-K_{W}\right|$, and $C$ be the base curve of the pencil $\left|-K_{W}\right|$. Then $\mathcal{B} \sim_{\mathbb{Q}}-k K_{W}$ by Theorem 2.2 the curve $C$ is irreducible, the inequality $C^{2}=-1 / 24$ holds on the normal surface $S$, and the equivalence $\left.\mathcal{B}\right|_{S} \sim_{\mathbb{Q}} k C$ holds, which is impossible by Lemmas 2.10 and 2.8. The claim of Proposition 15.1 is proved.

\section{Case $n=19$, hypersurface of Degree 12 in $\mathbb{P}(1,2,3,3,4)$.}

We use the notations and assumptions of Section [3. Let $n=19$. Then $X$ is a general hypersurface in $\mathbb{P}(1,2,3,3,4)$ of degree 12 , the singularities of the hypersurface $X$ consist of the points $O_{1}, O_{2}, O_{3}$ that are quotient singularities of type $\frac{1}{2}(1,1,1)$, the points $P_{1}, P_{2}, P_{3}, P_{4}$ that are quotient singularities of type $\frac{1}{3}(1,1,2)$, and the equality $-K_{X}^{3}=1 / 6$ holds.

It follows from Example 1.7 that for every $i \in\{1,2,3,4\}$ there is a commutative diagram

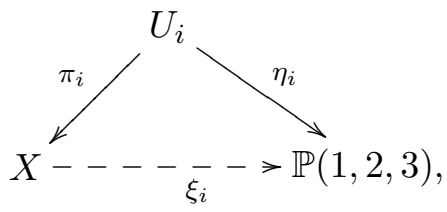

where $\xi_{i}$ is a projection, $\pi_{i}$ is the blow up of $P_{i}$ with weights $(1,1,2)$, and $\eta_{i}$ is an elliptic fibration.

Proposition 16.1. There is a commutative diagram

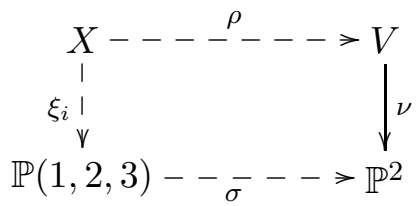

for some $i \in\{1,2,3,4\}$, where $\sigma$ is a birational map.

Proof. The set $\mathbb{C S}\left(X, \frac{1}{k} \mathcal{M}\right)$ contains the point $P_{i}$ for some $i \in\{1,2,3,4\}$. Let $\mathcal{D}$ be the proper transform of the linear system $\mathcal{M}$ on the variety $U_{i}$. Then $\mathcal{D} \sim_{\mathbb{Q}}-k K_{U_{i}}$ by Theorem 2.2. which implies the existence of the commutative diagram 16.2.

\section{Case $n=20$, hypersurface of Degree 13 in $\mathbb{P}(1,1,3,4,5)$.}

We use the notations and assumptions of Section 3 . Let $n=20$. Then $X$ is a general hypersurface in $\mathbb{P}(1,1,3,4,5)$ of degree 13 , the equality $-K_{X}^{3}=13 / 60$ holds, and the singularities of the hypersurface $X$ consist of the point $P_{1}$ that is a singularity of type $\frac{1}{3}(1,1,2)$, the point $P_{2}$ that is a singularity of type $\frac{1}{4}(1,1,3)$, and the point $P_{3}$ that is a singularity of type $\frac{1}{5}(1,1,4)$. There is a commutative diagram

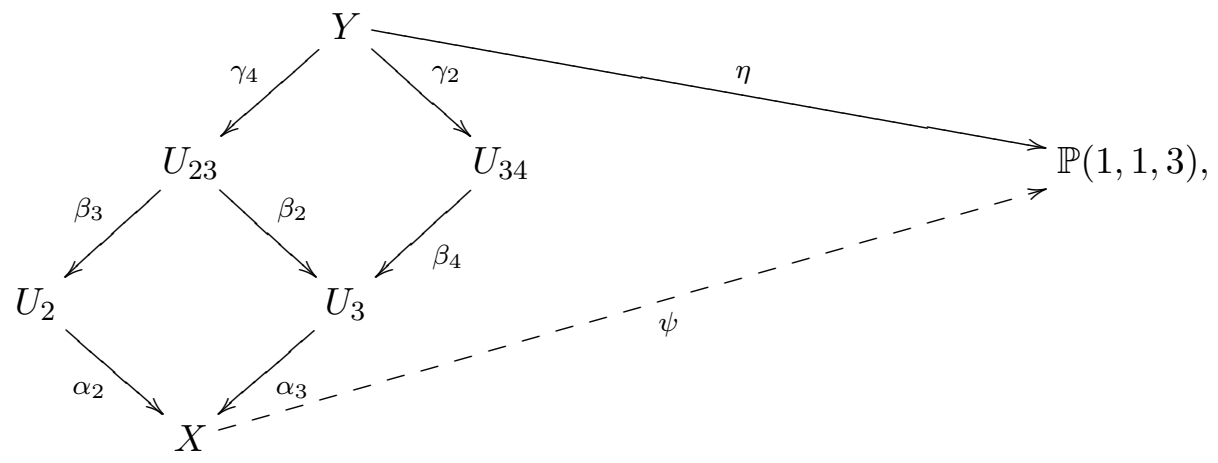

where $\psi$ is a projection, $\alpha_{2}$ is the weighted blow up of $P_{2}$ with weights $(1,1,3), \alpha_{3}$ is the weighted blow up of $P_{3}$ with weights $(1,1,4), \beta_{3}$ is the weighted blow up with weights $(1,1,4)$ of the proper transform of the point $P_{3}$ on $U_{2}, \beta_{2}$ is the weighted blow up with weights $(1,1,3)$ of the proper 
transform of the point $P_{2}$ on the variety $U_{3}, \beta_{4}$ is the weighted blow up with weights $(1,1,3)$ of the singular point of $U_{3}$ that is contained in the exceptional divisor of $\alpha_{3}, \gamma_{2}$ is the weighted blow up with weights $(1,1,3)$ of the proper transform of $P_{2}$ on $U_{34}, \gamma_{4}$ is the weighted blow up with weights $(1,1,3)$ of the singular point of the variety $U_{23}$ that is contained in the exceptional divisor of the morphism $\beta_{3}$, and $\eta$ is an elliptic fibration.

Remark 17.1. The divisors $-K_{U_{2}},-K_{U_{3}},-K_{U_{23}}$ and $-K_{U_{34}}$ are nef and big.

There is a commutative diagram

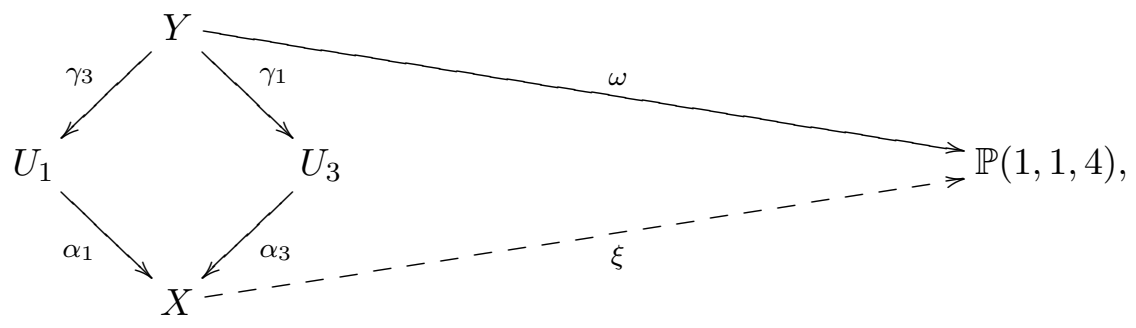

where $\xi$ is a projection, $\alpha_{1}$ is the blow up of $P_{1}$ with weights $(1,1,2), \alpha_{3}$ is the weighted blow up of the point $P_{3}$ weights $(1,1,4), \gamma_{3}$ is the weighted blow up with weights $(1,1,4)$ of the proper transform of $P_{3}$ on the variety $U_{1}, \gamma_{1}$ is the weighted blow up with weights $(1,1,2)$ of the proper transform of the point $P_{1}$ on the variety $U_{3}$, and $\omega$ is an elliptic fibration.

Remark 17.2. The divisor $-K_{U_{1}}$ is nef and big.

In the rest of the section we prove the following result.

Proposition 17.3. Either there is a commutative diagram

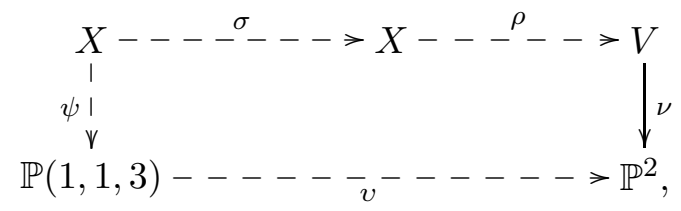

or there is a commutative diagram

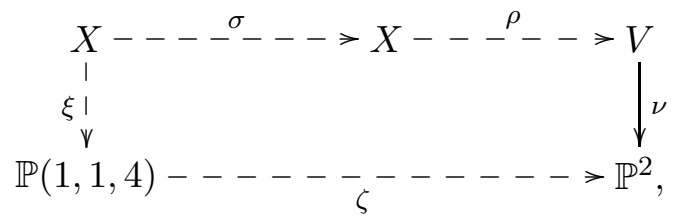

where $\sigma, v$ and $\zeta$ are birational maps.

It follows from Theorem 3.5 and Lemma 3.14 that $\mathbb{C S}\left(X, \frac{1}{k} \mathcal{M}\right) \subseteq\left\{P_{1}, P_{2}, P_{3}\right\}$.

Lemma 17.6. Suppose that $\left\{P_{1}, P_{3}\right\} \subseteq \mathbb{C S}\left(X, \frac{1}{k} \mathcal{M}\right)$. Then the commutative diagram 17.5 exists.

Proof. Let $\mathcal{B}$ be the proper transform of the linear system $\mathcal{M}$ on the variety $Y$, and $S$ be a general surface in $\mathcal{B}$. Then $S \sim_{\mathbb{Q}}-k K_{Y}$ by Theorem 2.2. We see that $S \cdot C=0$, where $C$ is a general fiber of $\omega$, which implies the existence of the commutative diagram 17.5 .

Lemma 17.7. The set $\mathbb{C S}\left(X, \frac{1}{k} \mathcal{M}\right)$ does not contain the set $\left\{P_{1}, P_{2}\right\}$.

Proof. Suppose that $\left\{P_{1}, P_{3}\right\} \subseteq \mathbb{C S}\left(X, \frac{1}{k} \mathcal{M}\right)$. Let $\pi: W \rightarrow U_{1}$ be the weighted blow up with weights $(1,1,3)$ of the proper transform of the point $P_{2}$ on the variety $U_{1}$, and $\mathcal{B}$ be the proper transform of the linear system $\mathcal{M}$ on the variety $W$. Then $\mathcal{B} \sim \mathbb{Q}-k K_{W}$ by Theorem 2.2.

The linear system $\left|-K_{W}\right|$ is a pencil, the base locus of the pencil $\left|-K_{W}\right|$ is the irreducible curve $\Delta$ such that the curve $\alpha_{1} \circ \pi(C)$ is cut on the hypersurface $X$ by the equations $z=y=0$.

Let $S$ be a sufficiently general surface of the linear system $\left|-K_{W}\right|, \bar{P}_{3}$ be the proper transform of the singular point $P_{3}$ on the variety $W$, and $\bar{P}_{5}$ and $\bar{P}_{6}$ be other singular points of $W$ such that $\alpha_{1} \circ \pi\left(\bar{P}_{5}\right)=P_{1}$ and $\alpha_{1} \circ \pi\left(\bar{P}_{6}\right)=P_{2}$. Then $\bar{P}_{5}$ is a quotient singularity of type $\frac{1}{2}(1,1,1)$ on the variety $W$, the point $\bar{P}_{6}$ is a quotient singularity of type $\frac{1}{3}(1,1,2)$ on the variety $W$, the 
surface $S$ is smooth outside of the points $\bar{P}_{3}, \bar{P}_{5}$ and $\bar{P}_{6}$, the singularities of the surface $S$ in the points $\bar{P}_{3}, \bar{P}_{5}$ and $\bar{P}_{6}$ are $\mathrm{Du}$ Val singularities of types $\mathbb{A}_{4}, \mathbb{A}_{1}$ and $\mathbb{A}_{2}$ respectively.

The equality $\Delta^{2}=-1 / 30$ holds on the surface $S$, but the equivalence $\left.\mathcal{B}\right|_{S} \sim_{\mathbb{Q}} k \Delta$ holds, which implies that $\left.\mathcal{B}\right|_{S}=k \Delta$. Now we can easily get a contradiction using Lemma 2.8 .

Lemma 17.8. The set $\mathbb{C S}\left(X, \frac{1}{k} \mathcal{M}\right)$ is not consists of the point $P_{i}$.

Proof. Suppose that $\mathbb{C} S\left(X, \frac{1}{k} \mathcal{M}\right)=\left\{P_{i}\right\}$. Let $\mathcal{D}$ be the proper transform of $\mathcal{M}$ on $U_{i}$. Then the equivalence $\mathcal{D} \sim_{\mathbb{Q}}-k K_{U_{i}}$ holds by Theorem 2.2. Moreover, it follows from Lemma 2.1 and the proof of Lemma 9.7 that the set $\mathbb{C S}\left(U_{i}, \frac{1}{k} \mathcal{D}\right)$ contains the singular point of $U_{i}$ that is the singular point of the exceptional divisor of the birational morphism $\alpha_{i}$.

Let $\pi: W \rightarrow U_{i}$ be the weighted blow up with weights $(1,1, i)$ of the singular point of the variety $U_{i}$ that is contained in the exceptional divisor of the morphism $\alpha_{i}$, and $\mathcal{B}$ be the proper transform of the linear system $\mathcal{M}$ on the variety $W$. Then $\mathcal{B} \sim_{\mathbb{Q}}-k K_{W}$ by Theorem 2.2.

Let $S$ be a sufficiently general surface of the pencil $\left|-K_{W}\right|$, and $\Delta$ be the unique base curve of the pencil $\left|-K_{W}\right|$. Then the surface $S$ is normal, but the curve $\Delta$ is irreducible, rational and smooth. Moreover, simple computations imply that the equality

$$
\Delta^{2}=\left\{\begin{array}{l}
-9 / 20 \quad i=1, \\
-1 / 30 \quad i=2, \\
0 \quad i=3
\end{array}\right.
$$

holds on the surface $S$. However, we have the equivalence $\left.\mathcal{B}\right|_{S} \sim_{\mathbb{Q}} k \Delta$, which implies (see the proof of Lemma 17.7) that the curve $\alpha_{i} \circ \pi(\Delta)$ is contained in the set $\mathbb{C S}\left(X, \frac{1}{k} \mathcal{M}\right)$ if $i \neq 3$.

Therefore, the equality $i=3$ holds.

Let $G$ be the exceptional divisor of $\alpha_{3}$, and $\bar{P}_{4}$ be the singular point of $U_{3}$ that is contained in divisor $G$. Then $\bar{P}^{4}$ is a quotient singularity of type $\frac{1}{4}(1,1,3)$ on the variety $U_{3}$, and it follows from Lemmas 2.3 and 2.4 that the set $\mathbb{C S}\left(U_{3}, \frac{1}{k} \mathcal{D}\right)$ consists of the singular point $\bar{P}_{4}$.

The variety $W$ is the variety $U_{34}$, and $\pi$ is the morphism $\beta_{4}$. Thus, the divisor $-K_{W}$ is nef and big. Therefore, it follows from Lemmas 2.1] and 2.3 that the set $\mathbb{C} \mathbb{S}\left(W, \frac{1}{k} \mathcal{B}\right)$ contains the singular point of the variety $W$ that is contained in the exceptional divisor of the $\pi$.

Let $\mu: Z \rightarrow W$ be the weighted blow up weights $(1,1,2)$ of the singular point of the variety $W$ that is contained in the exceptional divisor of the morphism $\pi$, and $\mathcal{P}$ be the proper transform of the linear system $\mathcal{M}$ on the variety $Z$. Then the equivalence $\mathcal{P} \sim_{\mathbb{Q}}-k K_{Z}$ holds by Theorem 2.2

Let $F$ be a sufficiently general surface of the pencil $\left|-K_{Z}\right|$, and $\Gamma$ be the unique base curve of the pencil $\left|-K_{Z}\right|$. Then the surface $F$ is irreducible and normal, but the curve $\Gamma$ is irreducible, rational and smooth. The equality $\Gamma^{2}=-1 / 24$ holds on $F$, but $\left.\mathcal{P}\right|_{S} \sim_{\mathbb{Q}} k \Gamma$, which easily leads to a contradiction using Lemmas 2.8 and 2.10.

To conclude the proof of Proposition 17.3 we may assume that $\mathbb{C S}\left(X, \frac{1}{k} \mathcal{M}\right)=\left\{P_{2}, P_{3}\right\}$.

Let $\mathcal{D}$ be the proper transform of $\mathcal{M}$ on $U_{23}$. Then $\mathcal{D} \sim_{\mathbb{Q}}-k K_{U_{23}}$ by Theorem [2.2] and it follows from Lemmas 2.1 and 2.3 that the set $\mathbb{C S}\left(U_{23}, \frac{1}{k} \mathcal{D}\right)$ contains either the singular point of the variety $U_{23}$ that is contained in the exceptional divisor of the morphism $\beta_{3}$, or the singular point of the variety $U_{23}$ that is contained in the exceptional divisor of $\beta_{2}$.

Lemma 17.9. The set $\mathbb{C S}\left(U_{23}, \frac{1}{k} \mathcal{D}\right)$ does not contain the singular point of the variety $U_{23}$ that is contained in the exceptional divisor of the morphism $\beta_{2}$.

Proof. Let $E$ be the exceptional divisor of the morphism $\beta_{2}$, and $\bar{P}_{6}$ be the singular point of the surface $E$. Then $\bar{P}_{6}$ is a quotient singularity of type $\frac{1}{3}(1,1,2)$ on $U_{23}$.

Suppose that the set $\mathbb{C S}\left(U_{23}, \frac{1}{k} \mathcal{D}\right)$ contains $\bar{P}_{6}$. Let $\pi: W \rightarrow U_{23}$ be the weighted blow up of the point $\bar{P}_{6}$ with weights $(1,1,2), \mathcal{B}$ be the proper transform of $\mathcal{M}$ on $W, S$ be a general surface of the pencil $\left|-K_{W}\right|$, and $\Delta$ be the base curve of the pencil $\left|-K_{W}\right|$. Then $S$ is normal, the curve $\Delta$ is irreducible, and $\left.\mathcal{B}\right|_{S} \sim_{\mathbb{Q}} k \Delta$ by Theorem 2.2. but the equality $\Delta^{2}=-1 / 24$ holds on the surface $S$, which contradicts Lemmas 2.10 and 2.8.

Hence, the set $\mathbb{C} \mathbb{S}\left(U_{23}, \frac{1}{k} \mathcal{D}\right)$ contains the singular point of the variety $U_{23}$ that is contained in the exceptional divisor of the morphism $\beta_{3}$. Thus, the existence of the commutative diagram 17.5 is easily implied by Theorem 2.2. The claim of Proposition 17.3 is proved. 
18. Case $n=23$, hypersurface of Degree 14 In $\mathbb{P}(1,2,3,4,5)$.

We use the notations and assumptions of Section [3. Let $n=23$. Then $X$ is a sufficiently general hypersurface in $\mathbb{P}(1,2,3,4,5)$ of degree 14 , the singularities of the hypersurface $X$ consist of the points $P_{1}, P_{2}$, and $P_{3}$ that are quotient singularities of type $\frac{1}{2}(1,1,1)$, the point $P_{4}$ that is a quotient singularity of type $\frac{1}{3}(1,1,2)$, the point $P_{5}$ that is a quotient singularity of type $\frac{1}{4}(1,1,3)$, the point $P_{6}$ that is quotient singularity of type $\frac{1}{5}(1,2,3)$, and the equality $-K_{X}^{3}=7 / 60$ holds.

There is a commutative diagram

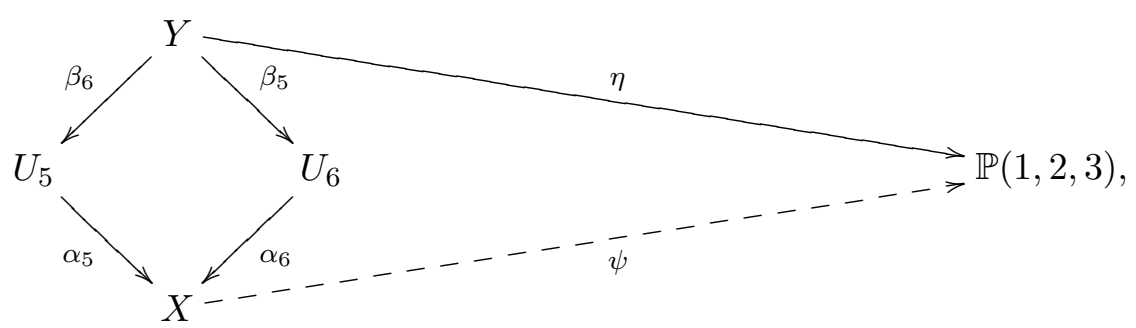

where $\psi$ is the natural projection, $\alpha_{5}$ is the weighted blow up of the singular point $P_{5}$ with weights $(1,1,3), \alpha_{6}$ is the weighted blow up of $P_{6}$ with weights $(1,2,3), \beta_{5}$ is the weighted blow up with weights $(1,1,3)$ of the proper transform of $P_{5}$ on the variety $U_{6}, \beta_{6}$ is the weighted blow up with weights $(1,2,3)$ of the proper transform of $P_{6}$ on $U_{5}$, and $\eta$ is an elliptic fibration.

In the rest of the section we prove the following result.

Proposition 18.1. The claim of Theorem 1.10 holds for $n=23$.

It follows from Theorem 3.5, Lemma 3.14 and Proposition 3.7 that $\mathbb{C S}\left(X, \frac{1}{k} \mathcal{M}\right) \subseteq\left\{P_{5}, P_{6}\right\}$.

Lemma 18.2. The set $\mathbb{C} S\left(X, \frac{1}{k} \mathcal{M}\right)$ contains the point $P_{6}$.

Proof. Suppose that $\mathbb{C S}\left(X, \frac{1}{k} \mathcal{M}\right)$ does not contain $P_{6}$. Then the set $\mathbb{C S}\left(X, \frac{1}{k} \mathcal{M}\right)$ consists of the point $P_{5}$. Let $\mathcal{D}_{5}$ be the proper transform of $\mathcal{M}$ on $U_{5}$. Then $\mathcal{D}_{5} \sim_{\mathbb{Q}}-k K_{U_{5}}$ by Theorem 2.2 but the set $\mathbb{C S}\left(U_{5}, \frac{1}{k} \mathcal{D}_{5}\right)$ is not empty by Lemma 2.1

Let $\bar{P}_{7}$ be the singular point of the variety $U_{5}$ that is contained in the exceptional divisor of the morphism $\alpha_{5}$. Then the point $\bar{P}_{7}$ is a quotient singularity of type $\frac{1}{3}(1,1,2)$ on $U_{5}$, and it follows from Lemma 2.3 that $\mathbb{C S}\left(U_{5}, \frac{1}{k} \mathcal{D}_{5}\right)$ contains $\bar{P}_{7}$. Let $\pi: U \rightarrow U_{5}$ be the weighted blow up of the point $\bar{P}_{7}$ with weights $(1,1,3)$. Then the linear system $\left|-2 K_{U}\right|$ is a proper transform of the pencil $\left|-2 K_{X}\right|$, and the base locus of the pencil $\left|-2 K_{U}\right|$ consists of a single irreducible curve $Z$ such that $\alpha_{5} \circ \pi(Z)$ is the unique base curve of the pencil $\left|-2 K_{X}\right|$.

Let $S$ be a sufficiently general surface of the pencil $\left|-2 K_{U}\right|$. Then the surface $S$ is normal, the surface $S$ contains the curve $Z$, and the inequality $Z^{2}<0$ holds on the surface $S$, because the inequality $-K_{U}^{3}<0$ holds. However, the equivalence $\left.\mathcal{B}\right|_{S} \sim_{\mathbb{Q}} k Z$ holds by Theorem [2.2] where $\mathcal{B}$ is the proper transform of $\mathcal{M}$ on $W$. It follows from Lemma 2.10 that

$$
\operatorname{Supp}(S) \cap \operatorname{Supp}(D)=\operatorname{Supp}(Z),
$$

where $D$ is a sufficiently general surface of the linear system $\mathcal{B}$, which contradicts Lemma 2.8 because the linear system $\mathcal{B}$ is not composed from a pencil.

It easily follows from Theorem 2.2 that the claim of Theorem 1.10 holds for $X$ whenever the set $\mathbb{C S}\left(X, \frac{1}{k} \mathcal{M}\right)$ contains the points $P_{5}$ and $P_{6}$. So, we may assume that $P_{5} \notin \mathbb{C S}\left(X, \frac{1}{k} \mathcal{M}\right)$.

Let $\mathcal{D}_{6}$ be the proper transform of $\mathcal{M}$ on $U_{6}$. Then $\mathcal{D}_{6} \sim_{\mathbb{Q}}-k K_{U_{6}}$ by Theorem [2.2] which implies that the set $\mathbb{C S}\left(U_{6}, \frac{1}{k} \mathcal{D}_{6}\right)$ is not empty by Lemma 2.1] Let $\bar{P}_{7}$ and $\bar{P}_{8}$ be the singular points of the variety $U_{6}$ that are quotient singularities of types $\frac{1}{3}(1,1,2)$ and $\frac{1}{2}(1,1,1)$ contained in the exceptional divisor of the morphism $\alpha_{6}$ respectively. Then the set $\mathbb{C} \mathbb{S}\left(U_{6}, \frac{1}{k} \mathcal{D}_{6}\right)$ contains either the point $\bar{P}_{7}$, or the point $\bar{P}_{8}$ by Lemma 2.3

Lemma 18.3. The set $\mathbb{C S}\left(U_{6}, \frac{1}{k} \mathcal{D}_{6}\right)$ does not contain the point $\bar{P}_{7}$.

Proof. Suppose that $\bar{P}_{7} \in \mathbb{C S}\left(U_{6}, \frac{1}{k} \mathcal{D}_{6}\right)$. Let $\gamma: W \rightarrow U_{6}$ be the weighted blow up of $\bar{P}_{7}$ with weights $(1,1,2)$, and $S$ be a general surface of in $\left|-2 K_{W}\right|$. Then the surface $S$ is irreducible 
and normal, the linear system $\left|-2 K_{W}\right|$ is the proper transform of the pencil $\left|-2 K_{X}\right|$, and the base locus of the pencil $\left|-2 K_{W}\right|$ consists of the irreducible curve $\Delta$ such that the equality

$$
\Delta^{2}=-2 K_{W}^{3}=-\frac{1}{6}
$$

holds on the surface $S$. Moreover, the equivalence $\left.\mathcal{B}\right|_{S} \sim_{\mathbb{Q}} k \Delta$ holds, where $\mathcal{B}$ is the proper transform of $\mathcal{M}$ on $W$, which contradicts Lemmas 2.10] and 2.8.

Therefore, the set $\mathbb{C} S\left(U_{6}, \frac{1}{k} \mathcal{D}_{6}\right)$ contains the point $\bar{P}_{8}$.

Remark 18.4. The linear system $\left|-3 K_{U_{6}}\right|$ is the proper transform of the linear system $\left|-3 K_{X}\right|$, the base locus of the linear system $\left|-3 K_{U_{6}}\right|$ consists of the irreducible fiber of $\psi \circ \alpha_{6}$ that passes through the singular point $\bar{P}_{8}$.

Let $\pi: U \rightarrow U_{6}$ be the weighted blow up of the point $\bar{P}_{8}$ with weights $(1,1,1), F$ be the exceptional divisor of the morphism $\pi, \mathcal{D}$ be the proper transform of the linear system $\mathcal{M}$ on the variety $U$, and $\mathcal{H}$ be the proper transform of the linear system $\left|-3 K_{U_{6}}\right|$ on $U$. Then

$$
\mathcal{D} \sim_{\mathbb{Q}}-k K_{U} \sim_{\mathbb{Q}} \pi^{*}\left(-k K_{U_{6}}\right)-\frac{k}{2} F
$$

by Theorem 2.2. The simple computations imply that

$$
\mathcal{H} \sim_{\mathbb{Q}} \pi^{*}\left(-3 K_{U_{6}}\right)-\frac{1}{2} F
$$

and the base locus of $\mathcal{H}$ consists of the irreducible curve $Z$ such that $\alpha_{6} \circ \pi(Z)$ is the base curve of the linear system $\left|-3 K_{X}\right|$. Moreover, the equality $S \cdot Z=1 / 12$ holds, where $S$ is a general surface of the linear system $\mathcal{H}$.

Let $D_{1}$ and $D_{2}$ be general surfaces of the linear system $\mathcal{D}$. Then

$$
-k^{2} / 4=\left(\pi^{*}\left(-3 K_{U_{6}}\right)-\frac{1}{2} F\right) \cdot\left(\pi^{*}\left(-k K_{U_{6}}\right)-\frac{k}{2} F\right)^{2}=\left(\pi^{*}\left(-3 K_{U_{6}}\right)-\frac{1}{2} F\right) \cdot D_{1} \cdot D_{2} \geqslant 0
$$

which is a contradiction. Hence, the claim of Proposition [18.1 is proved.

$$
\text { 19. Case } n=25 \text {, hypersurface of Degree } 15 \text { In } \mathbb{P}(1,1,3,4,7) \text {. }
$$

We use the notations and assumptions of Section 3. Let $n=25$. Then $X$ is a sufficiently general hypersurface in $\mathbb{P}(1,1,3,4,7)$ of degree 15 , the equality $-K_{X}^{3}=5 / 28$ holds, and the singularities of $X$ consist of the point $P_{1}$ that is a quotient singularity of type $\frac{1}{4}(1,1,3)$, and the point $P_{2}$ that is a quotient singularity of type $\frac{1}{7}(1,3,4)$.

Proposition 19.1. The claim of Theorem 1.10 holds for $n=25$.

There is a commutative diagram

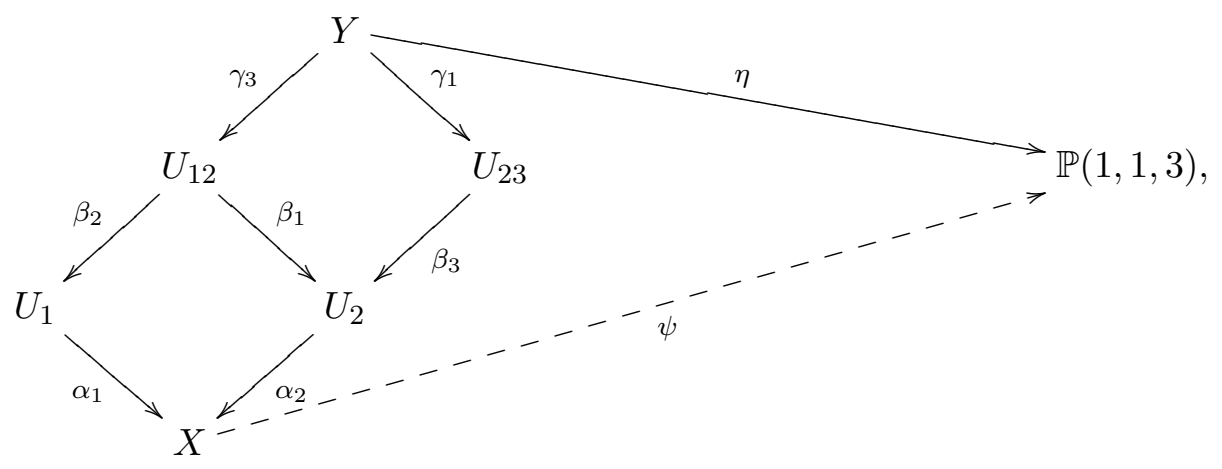

where $\psi$ is a projection, $\alpha_{1}$ is the weighted blow up of $P_{1}$ with weights $(1,1,3), \alpha_{2}$ is the weighted blow up of $P_{2}$ with weights $(1,3,4), \beta_{2}$ is the weighted blow up with weights $(1,3,4)$ of the proper transform of $P_{2}$ on $U_{1}, \beta_{1}$ is the weighted blow up with weights $(1,1,3)$ of the proper transform of the point $P_{1}$ on the variety $U_{2}, \beta_{3}$ is the weighted blow up with weights $(1,1,3)$ of the singular point of the variety $U_{2}$ that is a quotient singularity of type $\frac{1}{4}(1,1,3)$ contained in the exceptional divisor of the morphism $\alpha_{2}, \gamma_{1}$ is the weighted blow up with weights $(1,1,3)$ of the proper transform of $P_{1}$ on $U_{23}, \gamma_{3}$ is the weighted blow up with weights $(1,1,3)$ of the point 
of the variety $U_{12}$ that is a quotient singularity of type $\frac{1}{4}(1,1,3)$ contained in the exceptional divisor of the morphism $\beta_{2}$, and $\eta$ is an elliptic fibration.

Remark 19.2. The divisors $-K_{U_{1}},-K_{U_{2}},-K_{U_{12}}$ and $-K_{U_{23}}$ are nef and big.

Let us prove Proposition 19.1. It follows from Theorem 3.5. Lemma 3.14 and Proposition 3.7 that $\mathbb{C S}\left(X, \frac{1}{k} \mathcal{M}\right) \subseteq\left\{P_{1}, P_{2}\right\}$. To conclude the proof of Proposition [19.1] we may assume that the singularities of the log pair $\left(X, \frac{1}{k} \mathcal{M}\right)$ are canonical (see Remark 3.4).

Lemma 19.3. The set $\mathbb{C S}\left(X, \frac{1}{k} \mathcal{M}\right)$ contains the point $P_{2}$.

Proof. Suppose that the $\mathbb{C S}\left(X, \frac{1}{k} \mathcal{M}\right)$ does not contain the point $P_{2}$. Let $\mathcal{D}_{1}$ be the proper transform of the $\mathcal{M}$ on the variety $U_{1}$. Then $\mathbb{C S}\left(X, \frac{1}{k} \mathcal{M}\right)=\left\{P_{1}\right\}$, and the set $\mathbb{C S}\left(U_{1}, \frac{1}{k} \mathcal{D}_{1}\right)$ is not empty by Lemma 2.1] because the equivalence $\mathcal{D}_{1} \sim_{\mathbb{Q}}-k K_{U_{1}}$ holds by Theorem [2.2.

Let $P_{5}$ be the singular point of the variety $U_{1}$ that is contained in the exceptional divisor of the morphism $\alpha_{1}$. Then the point $P_{5}$ is a quotient singularity of type $\frac{1}{3}(1,1,2)$ on $U_{1}$, and it follows from Lemma 2.3 that $\mathbb{C S}\left(U_{1}, \frac{1}{k} \mathcal{D}_{1}\right)$ contains the point $P_{5}$.

Let $\pi: W \rightarrow U_{1}$ be the blow up of the point $P_{5}$ with weights $(1,1,2)$, and $S$ be a sufficiently general surface of the pencil $\left|-K_{W}\right|$. Then the surface $S$ is irreducible and normal, and the base locus of the pencil $\left|-K_{W}\right|$ consists of the irreducible curve $\Delta$ such that

$$
\Delta^{2}=-K_{W}^{3}=-\frac{1}{14}
$$

on the surface $S$, but $\left.\mathcal{B}\right|_{S} \sim_{\mathbb{Q}} k \Delta$, where $\mathcal{B}$ is the proper transform of the linear system $\mathcal{M}$ on the variety $W$. Therefore, we have $\left.\mathcal{B}\right|_{S}=k \Delta$, which implies that

$$
\operatorname{Supp}(S) \cap \operatorname{Supp}(D)=\operatorname{Supp}(\Delta),
$$

where $D$ is a general surface in $\mathcal{B}$. The latter contradicts Lemma 2.8 .

Let $G$ be the exceptional divisor of $\alpha_{2}, \mathcal{D}_{2}$ be the proper transform of the linear system $\mathcal{M}$ on the variety $U_{2}, \bar{P}_{1}$ be the proper transform of $P_{1}$ on $U_{2}$, and $\bar{P}_{3}$ and $\bar{P}_{4}$ are the singular points of the variety $U_{2}$ that are quotient singularities of types $\frac{1}{4}(1,1,3)$ and $\frac{1}{3}(1,1,2)$ contained in the exceptional divisor $G$ respectively. Then $G \cong \mathbb{P}(1,3,4)$, the points $\bar{P}_{3}$ and $\bar{P}_{4}$ are singular points of the surface $G$, and $\mathcal{D}_{2} \sim_{\mathbb{Q}}-k K_{U_{2}}$ by Theorem 2.2. Hence, the set $\mathbb{C} \mathbb{S}\left(U_{2}, \frac{1}{k} \mathcal{D}_{2}\right)$ is not empty by Lemma 2.1. Moreover, the proof of Lemma 19.3 implies that $\mathbb{C} \mathbb{S}\left(U_{2}, \frac{1}{k} \mathcal{D}_{2}\right) \neq\left\{\bar{P}_{1}\right\}$.

Lemma 19.4. The set $\mathbb{C S}\left(U_{2}, \frac{1}{k} \mathcal{D}_{2}\right)$ does not contain both points $\bar{P}_{3}$ and $\bar{P}_{4}$.

Proof. Suppose that $\left\{\bar{P}_{3}, \bar{P}_{4}\right\} \subseteq \mathbb{C S}\left(U_{2}, \frac{1}{k} \mathcal{D}_{2}\right)$. Let $\pi: W \rightarrow U_{2}$ be a composition of the weighted blow ups of the points $\bar{P}_{3}$ and $\bar{P}_{4}$ with weights $(1,1,3)$ and $(1,1,2)$ respectively, and $\mathcal{B}$ be the proper transform of $\mathcal{M}$ on the variety $W$. Then $\mathcal{B} \sim_{\mathbb{Q}}-k K_{W}$ by Theorem 2.2 .

Let $S$ be a general surface of the pencil $\left|-K_{W}\right|$. Then the surface $S$ is irreducible and normal, but the base locus of the pencil $\left|-K_{W}\right|$ consists of the irreducible curves $C$ and $L$ such that the curve $\alpha_{2} \circ \pi(C)$ is the unique base curve of the pencil $\left|-K_{X}\right|$, the curve $\pi(L)$ is contained in the surface $G$, and $\pi(L)$ is the unique curve in $\left|\mathcal{O}_{\mathbb{P}(1,3,4)}(1)\right|$. We have

$$
\left.\mathcal{B}\right|_{S} \sim_{\mathbb{Q}}-\left.\left.k K_{W}\right|_{S} \sim_{\mathbb{Q}} k S\right|_{S}=k C+k L
$$

but the intersection form of $L$ and $C$ on $S$ is negatively defined, and Lemma 2.10 implies that

$$
\operatorname{Supp}(S) \cap \operatorname{Supp}(D)=\operatorname{Supp}(C) \cup \operatorname{Supp}(L),
$$

where $D$ is a general surface in $\mathcal{B}$, which is impossible by Lemma 2.8

Thus, we have $\mathbb{C S}\left(U_{2}, \frac{1}{k} \mathcal{D}_{2}\right) \subsetneq\left\{\bar{P}_{1}, \bar{P}_{3}, \bar{P}_{4}\right\}$ by Lemma 2.3 .

Lemma 19.5. The set $\mathbb{C} \mathbb{S}\left(U_{2}, \frac{1}{k} \mathcal{D}_{2}\right)$ contains either the point $\bar{P}_{1}$, or the point $\bar{P}_{4}$.

Proof. Suppose that the set $\mathbb{C S}\left(U_{2}, \frac{1}{k} \mathcal{D}_{2}\right)$ does not contain neither the singular point $\bar{P}_{1}$, nor the singular point $\bar{P}_{4}$. Then the set $\mathbb{C} \mathbb{S}\left(U_{2}, \frac{1}{k} \mathcal{D}_{2}\right)$ consists of the point $\bar{P}_{3}$.

The linear system $\left|-K_{U_{2}}\right|$ is the proper transform of the pencil $\left|-K_{X}\right|$, and the base locus of the pencil $\left|-K_{U_{2}}\right|$ consists of the irreducible curves $L$ and $\Delta$ such that $\alpha_{2}(\Delta)$ is the unique 
base curve of the pencil $\left|-K_{X}\right|$, the curve $L$ is contained in the divisor $G$, the curve $L$ is the unique curve of the linear system $\left|\mathcal{O}_{\mathbb{P}(1,3,4)}(1)\right|$.

Let $\tilde{P}_{6}$ be the singular points of the variety $U_{23}$ that is contained in the exceptional divisor of $\beta_{3}$, and $\mathcal{D}_{23}$ be the proper transform of $\mathcal{M}$ on $U_{23}$. Then $\mathcal{D}_{23} \sim_{\mathbb{Q}}-k K_{U_{23}}$ by Theorem 2.2 , and it follows from Lemmas 2.1 and 2.3 the the set $\mathbb{C S}\left(U_{23}, \frac{1}{k} \mathcal{D}_{23}\right)$ contains the point $\tilde{P}_{6}$ that is a quotient singularity of type $\frac{1}{3}(1,1,2)$ on the variety $U_{23}$.

Let $\pi: W \rightarrow U_{23}$ be the weighted blow up of $\tilde{P}_{6}$ with weights $(1,1,2), \mathcal{D}$ be the proper transform of $\mathcal{M}$ on the variety $W$, and $\bar{L}$ and $\bar{\Delta}$ be the proper transforms of $L$ and $\Delta$ on the variety $W$ respectively. Then $\mathcal{D} \sim_{\mathbb{Q}}-k K_{W}$ by Theorem 2.2 , the pencil $\left|-K_{W}\right|$ is the proper transform of the pencil $\left|-K_{U_{2}}\right|$, and the base locus of $\left|-K_{W}\right|$ consists of $\bar{L}$ and $\bar{\Delta}$.

Let $S$ be a general surface of the pencil $\left|-K_{W}\right|$. Then the surface $S$ is irreducible and normal, the equivalence $\left.\mathcal{D}\right|_{S} \sim_{\mathbb{Q}} k \bar{\Delta}+k \bar{L}$ holds, but the equalities

$$
\bar{\Delta}^{2}=-7 / 12, \bar{L}^{2}=-5 / 6, \bar{\Delta} \cdot \bar{L}=2 / 3
$$

hold on the surface $S$. Therefore, the intersection form of the curves $\bar{\Delta}$ and $\bar{L}$ on normal the surface $S$ is negatively defined, which contradicts Lemmas 2.10] and 2.8

The hypersurface $X$ can be given by the equation

$w^{2} y+w t^{2}+w t f_{4}(x, y, z)+w f_{8}(x, y, z)+t f_{11}(x, y, z)+f_{15}(x, y, z)=0 \subset \operatorname{Proj}(\mathbb{C}[x, y, z, t, w])$,

where $\operatorname{wt}(x)=1, \operatorname{wt}(y)=1, \operatorname{wt}(z)=3, \operatorname{wt}(t)=4, \operatorname{wt}(w)=7$, and $f_{i}(x, y, t)$ is a sufficiently general quasihomogeneous polynomial of degree $i$.

Remark 19.6. Suppose that the set $\mathbb{C} \mathbb{S}\left(U_{2}, \frac{1}{k} \mathcal{D}_{2}\right)$ contains both points $\bar{P}_{1}$ and $\bar{P}_{3}$. Then the claim of Theorem 2.2 easily implies the existence of the commutative diagram

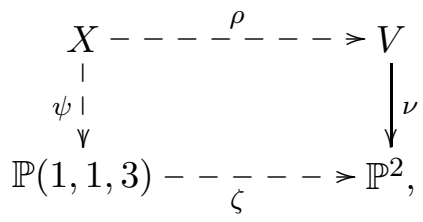

where $\zeta$ is a birational map.

Therefore, we may assume that set $\mathbb{C S}\left(U_{2}, \frac{1}{k} \mathcal{D}_{2}\right)$ does not contains both points $\bar{P}_{1}$ and $\bar{P}_{3}$.

Lemma 19.7. The set $\mathbb{C} \mathbb{S}\left(U_{2}, \frac{1}{k} \mathcal{D}_{2}\right)$ contains the point $\bar{P}_{1}$.

Proof. Suppose that $\bar{P}_{1} \notin \mathbb{C S}\left(U_{2}, \frac{1}{k} \mathcal{D}_{2}\right)$. Then $\mathbb{C S}\left(U_{2}, \frac{1}{k} \mathcal{D}_{2}\right)=\left\{\bar{P}_{4}\right\}$.

Let $\pi: W \rightarrow U_{2}$ be the weighted blow up of the point $\bar{P}_{4}$ with weights $(1,1,2), E$ be the exceptional divisor of the morphism $\pi$, and $\bar{G}$ and $\mathcal{B}$ be proper transforms of the divisor $G$ and the linear system $\mathcal{M}$ on the variety $W$ respectively. Then it follows from Theorem 2.2 that the equivalence $\mathcal{B} \sim \mathbb{Q}-k K_{W}$ holds, but the proof of Lemma 19.5 implies that the set $\mathbb{C S}\left(W, \frac{1}{k} \mathcal{B}\right)$ does not contain the singular point of the variety $W$ that is contained in the exceptional divisor of the morphism $\pi$. Therefore, the singularities of the $\log$ pair $\left(W, \frac{1}{k} \mathcal{B}\right)$ are terminal by Lemma 2.3

Let $S_{x}, S_{y}, S_{z}, S_{t}$ and $S_{w}$ be proper transforms on the variety $W$ of the surfaces that are cut on the variety $X$ by the equations $x=0, y=0, z=0, t=0$ and $w=0$ respectively. Then

$$
\left\{\begin{array}{l}
S_{x} \sim_{\mathbb{Q}}\left(\alpha_{2} \circ \pi\right)^{*}\left(-K_{X}\right)-\frac{3}{7} E-\frac{1}{7} \bar{G}, \\
S_{y} \sim_{\mathbb{Q}}\left(\alpha_{2} \circ \pi\right)^{*}\left(-K_{X}\right)-\frac{10}{7} E-\frac{8}{7} \bar{G}, \\
S_{z} \sim_{\mathbb{Q}}\left(\alpha_{2} \circ \pi\right)^{*}\left(-3 K_{X}\right)-\frac{2}{7} E-\frac{3}{7} \bar{G}, \\
S_{t} \sim_{\mathbb{Q}}\left(\alpha_{2} \circ \pi\right)^{*}\left(-4 K_{X}\right)-\frac{5}{7} E-\frac{4}{7} \bar{G}, \\
S_{w} \sim_{\mathbb{Q}}\left(\alpha_{2} \circ \pi\right)^{*}\left(-7 K_{X}\right) .
\end{array}\right.
$$


The base locus of the pencil $\left|-K_{W}\right|$ consists of the irreducible curves $C$ and $L$ such that the curve $\alpha_{2} \circ \pi(C)$ is cut by the equations $x=y=0$ on the hypersurface $X$, the curve $\pi(L)$ is contained in the surface $G$, and the curve $\pi(L)$ is contained in the linear system $\left|\mathcal{O}_{\mathbb{P}(1,3,4)}(1)\right|$.

The equivalences 19.8 implies that the rational functions $y / x, z y / x^{4}, t y / x^{5}$ and $w y^{3} / x^{10}$ are contained in the linear system $\left|a S_{x}\right|$, where $a=1,4,5$ and 10 respectively. Therefore, the linear system $\left|-20 K_{W}\right|$ induces the birational map $\chi: W \rightarrow X^{\prime}$, where $X^{\prime}$ is a hypersurface with canonical singularities in $\mathbb{P}(1,1,4,5,10)$ of degree 20 . In particular, the divisor $-K_{W}$ is big.

It follows from [13] that there is a composition of antiflips $\zeta: W \rightarrow Z$ such that the rational map $\zeta$ is regular outside of $C \cup L$, and the divisor divisor $-K_{Z}$ is nef. Let $\mathcal{P}$ be the proper transform of the linear system $\mathcal{M}$ on $Z$. Then the singularities of $\log$ pair $\left(Z, \frac{1}{k} \mathcal{P}\right)$ are terminal, because $\zeta$ is a log-flop with respect to the $\log$ pair $\left(W, \frac{1}{k} \mathcal{B}\right)$, which has terminal singularities, but it follows from Lemma 2.1 that the singularities of $\left(Z, \frac{1}{k} \mathcal{P}\right)$ are not terminal singularities.

Hence, the set $\mathbb{C S}\left(U_{2}, \frac{1}{k} \mathcal{D}_{2}\right)$ consists of the points $\bar{P}_{1}$ and $\bar{P}_{4}$.

Let $\pi: W \rightarrow U_{2}$ be a composition of the weighted blow ups of the points $\bar{P}_{1}$ and $\bar{P}_{4}$ with weights $(1,1,3)$ and $(1,1,2)$ respectively, $\bar{G}$ and $\mathcal{B}$ be the proper transforms of $G$ and $\mathcal{M}$ on the variety $W$ respectively, and $F$ and $E$ be exceptional divisors of the morphism $\pi$ that dominates the points $\bar{P}_{1}$ and $\bar{P}_{4}$ respectively. Then the equivalence $\mathcal{B} \sim_{\mathbb{Q}}-k K_{W}$ holds by Theorem 2.2 but it follows from the proof of Lemma 19.5 that the singularities of $\left(W, \frac{1}{k} \mathcal{B}\right)$ are terminal.

Let $S_{x}, S_{y}, S_{z}, S_{t}$ and $S_{w}$ be the proper transforms on the variety $W$ of the surfaces that are cut on $X$ by the equations $x=0, y=0, z=0, t=0$ and $w=0$ respectively. Then

$$
\left\{\begin{array}{l}
S_{x} \sim_{\mathbb{Q}}\left(\alpha_{2} \circ \pi\right)^{*}\left(-K_{X}\right)-\frac{3}{7} E-\frac{1}{7} \bar{G}-\frac{1}{4} F \sim_{\mathbb{Q}}-K_{W}, \\
S_{y} \sim_{\mathbb{Q}}\left(\alpha_{2} \circ \pi\right)^{*}\left(-K_{X}\right)-\frac{10}{7} E-\frac{8}{7} \bar{G}-\frac{1}{4} F \\
S_{z} \sim_{\mathbb{Q}}\left(\alpha_{2} \circ \pi\right)^{*}\left(-3 K_{X}\right)-\frac{2}{7} E-\frac{3}{7} \bar{G}-\frac{3}{4} F \\
S_{t} \sim_{\mathbb{Q}}\left(\alpha_{2} \circ \pi\right)^{*}\left(-4 K_{X}\right)-\frac{5}{7} E-\frac{4}{7} \bar{G}, \\
S_{w} \sim_{\mathbb{Q}}\left(\alpha_{2} \circ \pi\right)^{*}\left(-7 K_{X}\right)-\frac{11}{4} F
\end{array}\right.
$$

which imply that the rational functions $y / x, z y / x^{4}, t w y^{4} / x^{15}$ and $w y^{3} / x^{10}$ are contained in the linear system $\left|a S_{x}\right|$, where $a=1,4,15$ and 10 respectively. The linear system $\left|-60 K_{W}\right|$ induces the birational map $\chi: W \rightarrow X^{\prime}$ such that the variety $X^{\prime}$ is a hypersurface in $\mathbb{P}(1,1,4,10,15)$ of degree 30. In particular, the divisor $-K_{W}$ is big. Now we can obtain a contradiction in the same was as in the proof of Lemma 19.7. The claim of Proposition 19.1] is proved.

\section{Case $n=26$, hypersurface of Degree 15 in $\mathbb{P}(1,1,3,5,6)$.}

We use the notations and assumptions of Section [3. Let $n=26$. Then $X$ is a sufficiently general hypersurface in $\mathbb{P}(1,1,3,5,6)$ of degree 15 , the equality $-K_{X}^{3}=1 / 6$ holds, and the singularities of the hypersurface $X$ consist of the points $P_{1}$ and $P_{2}$ that are quotient singularities of type $\frac{1}{3}(1,1,2)$, and the point $P_{3}$ that is a quotient singularity of type $\frac{1}{6}(1,1,5)$.

There is a commutative diagram

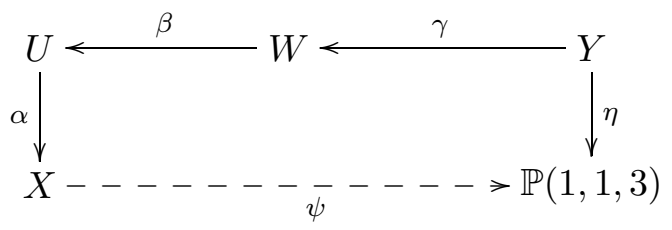

where $\psi$ is a projection, $\alpha$ is the weighted blow up of $P_{3}$ with weights $(1,1,5), \beta$ is the weighted blow up with weights $(1,1,4)$ of the singular point of the variety $U$ that is contained in the exceptional divisor of the morphism $\alpha, \gamma$ is the weighted blow up with weights $(1,1,3)$ of the singular point of the variety $W$ that is contained in the exceptional divisor of the morphism $\beta$, and $\eta$ is an elliptic fibration. 
There is a commutative diagram

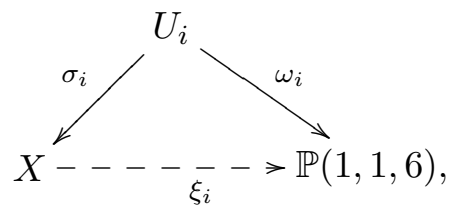

where $\xi_{i}$ is a projection, $\sigma_{i}$ is the weighted blow up of the point $P_{i}$ with weights $(1,1,2)$, and $\omega_{i}$ is an elliptic fibration, which is induced by the linear system $\left|-6 K_{U_{i}}\right|$.

It follows from [6] that the group $\operatorname{Bir}(X)$ is generated by biregular automorphisms of the hypersurface $X$ and a birational involution $\tau \in \operatorname{Bir}(X)$ such that $\psi \circ \tau=\psi$ and $\xi_{1} \circ \tau=\xi_{2}$.

In the rest of the section we prove the following result.

Proposition 20.1. Either there is a commutative diagram

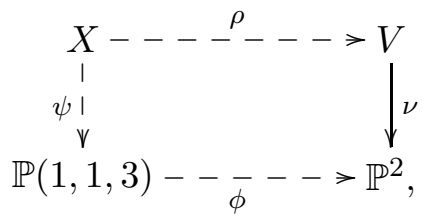

or there is a commutative diagram

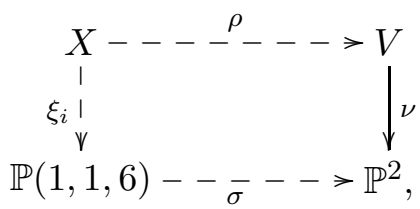

where $\phi$ and $\sigma$ are birational maps, and $i=1$ or $i=2$.

It follows from Theorem 3.5 and Lemma 3.14 that $\mathbb{C S}\left(X, \frac{1}{k} \mathcal{M}\right) \subseteq\left\{P_{1}, P_{2}, P_{3}\right\}$.

Lemma 20.4. Suppose that $P_{i} \in \mathbb{C} S\left(X, \frac{1}{k} \mathcal{M}\right)$. Then the commutative diagram 20.3 exists.

Proof. Let $\mathcal{B}$ be the proper transform of $\mathcal{M}$ on $U_{i}$. Then $\mathcal{B} \sim_{\mathbb{Q}}-k K_{U_{i}}$ by Theorem 2.2 which implies the existence of the commutative diagram 20.3 .

Therefore, we may assume that the set $\mathbb{C} S\left(X, \frac{1}{k} \mathcal{M}\right)$ consists of the point $P_{3}$.

Let $\mathcal{D}$ be the proper transform of the linear system $\mathcal{M}$ on the variety $U$, and $\bar{P}_{4}$ be the singular point of $U$ that is contained in the exceptional divisor of the morphism $\alpha$. Then $\mathcal{D} \sim_{\mathbb{Q}}-k K_{U}$ by Theorem 2.2, and the set $\mathbb{C} S\left(U, \frac{1}{k} \mathcal{D}\right)$ consists of the point $\bar{P}_{4}$ by Lemmas 2.3 and 2.4.

Let $\mathcal{H}$ be the proper transform of $\mathcal{M}$ on $W$. Then $\mathcal{H} \sim_{\mathbb{Q}}-k K_{W}$ by Theorem 2.2. but it follows from Lemmas 2.1 and 2.3 that $\mathbb{C S}\left(W, \frac{1}{k} \mathcal{H}\right)$ contains the singular point of $W$ that is contained in the exceptional divisor of $\beta$. The existence of the diagram 20.2 follows from Theorem 2.2 ,

\section{Case $n=27$, hypersurface of Degree 15 IN $\mathbb{P}(1,2,3,5,5)$.}

We use the notations and assumptions of Section 3 Let $n=27$. Then $X$ is a general hypersurface in $\mathbb{P}(1,2,3,5,5)$ of degree 15 , whose singularities consist of the point $O$ that is a singularity of type $\frac{1}{2}(1,1,1)$, and the points $P_{1}, P_{2}$ and $P_{3}$ that are singularities of type $\frac{1}{5}(1,2,3)$.

There is a commutative diagram

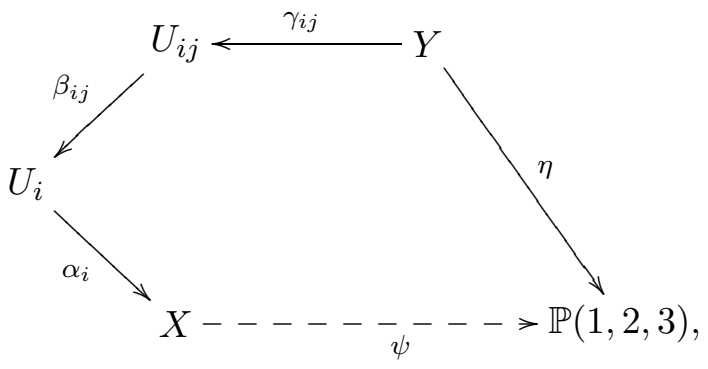


where $\psi$ is a projection, $\alpha_{i}$ is the blow up of $P_{i}$ with weights $(1,2,3), \beta_{i j}$ is the weighted blow up with weights $(1,2,3)$ of the proper transform of $P_{j}$ on the variety $U_{i}, \gamma_{i j}$ is the weighted blow up with weights $(1,2,3)$ of the proper transform of $P_{k}$ on $U_{i j}$, and $\eta$ is and elliptic fibration, where $i \neq j$ and $k \notin\{i, j\}$.

Proposition 21.1. The claim of Theorem 1.10 holds for $n=27$.

Let us prove Proposition 21.1. It follows from Lemma 3.14 and Proposition 3.7 that

$$
\varnothing \neq \mathbb{C S}\left(X, \frac{1}{k} \mathcal{M}\right) \subseteq\left\{P_{1}, P_{2}, P_{3}\right\}
$$

Remark 21.2. In the case $\mathbb{C S}\left(X, \frac{1}{k} \mathcal{M}\right)=\left\{P_{1}, P_{2}, P_{3}\right\}$, the claim of Theorem 1.10 holds for the hypersurface $X$ by Theorem 2.2

We may assume that the set $\mathbb{C} S\left(X, \frac{1}{k} \mathcal{M}\right)$ contains $P_{1}$ and does not contain $P_{3}$.

Lemma 21.3. The set $\mathbb{C} S\left(X, \frac{1}{k} \mathcal{M}\right)$ contains the point $P_{2}$.

Proof. Suppose that $\mathbb{C S}\left(X, \frac{1}{k} \mathcal{M}\right)$ does not contains the point $P_{2}$. Then $\mathbb{C S}\left(X, \frac{1}{k} \mathcal{M}\right)$ consists of the point $P_{1}$. Let $\mathcal{D}_{1}$ be the proper transform of $\mathcal{M}$ on $U_{1}$. Then Theorem 2.2 implies that the equivalence $\mathcal{D}_{1} \sim_{\mathbb{Q}}-k K_{U_{1}}$ holds. The set $\mathbb{C} \mathbb{S}\left(U_{1}, \frac{1}{k} \mathcal{D}_{1}\right)$ is not empty by Lemma 2.1

Let $G$ be is the exceptional divisor of $\alpha_{1}$, and $O$ and $Q$ are the singular points of $G$ that are quotient singularities of types $\frac{1}{3}(1,1,2)$ and $\frac{1}{2}(1,1,1)$ on $U_{1}$ respectively. Then it follows from the claim of Lemma 2.3 that $\mathbb{C S}\left(U_{1}, \frac{1}{k} \mathcal{D}_{1}\right)$ contains either the point $O$, or the point $Q$.

The linear system $\left|-2 K_{U_{1}}\right|$ is a pencil, and the base locus of the pencil $\left|-2 K_{U_{1}}\right|$ consists of the irreducible curve $C$ such that the curve $C$ passes through the point $O$, and $C$ is contracted by the rational map $\psi \circ \alpha_{1}$ to a singular point of the surface $\mathbb{P}(1,2,3)$.

Suppose that the set $\mathbb{C} S\left(U_{1}, \frac{1}{k} \mathcal{D}_{1}\right)$ contains the point $O$. Let $\pi: W \rightarrow U_{1}$ be the weighted blow up of $O$ with weights $(1,1,2), \mathcal{B}$ be the proper transform of $\mathcal{M}$ on $W$, and $\bar{C}$ be the proper transform of $C$ on $W$. Then $\mathcal{B} \sim_{\mathbb{Q}}-k K_{W}$ by Theorem 2.2] the linear system $\left|-2 K_{W}\right|$ is the proper transform of the pencil $\left|-2 K_{U_{1}}\right|$, and the base locus of $\left|-2 K_{W}\right|$ consists of $\bar{C}$.

Let $S$ be a general surface in $\left|-2 K_{W}\right|$. Then $\left.\mathcal{B}\right|_{S} \sim_{\mathbb{Q}} k \bar{C}$, but on the surface $S$, the strict inequality $\bar{C}^{2}<0$ holds. We have $\operatorname{Supp}(S) \cap \operatorname{Supp}(D)=\operatorname{Supp}(\bar{C})$, where $D$ is a general surface of the linear system $\mathcal{B}$, which is impossible by Lemma [2.8.

Hence, the set $\mathbb{C} \mathbb{S}\left(U_{1}, \frac{1}{k} \mathcal{D}_{1}\right)$ contains the point $Q$.

Let $\zeta: U \rightarrow U_{1}$ be the weighted blow up of the point $Q$ with weights $(1,1,1), F$ be the exceptional divisor of $\zeta, \mathcal{H}$ be the proper transform of $\mathcal{M}$ on $U$, and $\mathcal{P}$ be the proper transform of the linear system $\left|-3 K_{U_{1}}\right|$ on $U$. Then $\mathcal{H} \sim_{\mathbb{Q}}-k K_{U}$ by Theorem 2.2, but

$$
\mathcal{P} \sim_{\mathbb{Q}} \zeta^{*}\left(-3 K_{U_{1}}\right)-\frac{1}{2} F,
$$

and the base locus of $\mathcal{P}$ consists of the irreducible curve $Z$ such that the curve $\alpha_{1} \circ \zeta(Z)$ is the unique base curve of the linear system $\left|-3 K_{X}\right|$. Therefore, we have

$$
\left(\zeta^{*}\left(-3 K_{U_{1}}\right)-\frac{1}{2} F\right) \cdot Z=\frac{1}{10}
$$

which implies that

$$
-\frac{3 k^{2}}{10}=\left(\zeta^{*}\left(-3 K_{U_{1}}\right)-\frac{1}{2} F\right) \cdot\left(\zeta^{*}\left(-k K_{U_{1}}\right)-\frac{k}{2} F\right)^{2}=\left(\zeta^{*}\left(-3 K_{U_{1}}\right)-\frac{1}{2} F\right) \cdot H_{1} \cdot H_{2} \geqslant 0,
$$

where $H_{1}$ and $H_{2}$ are general surfaces of the linear system $\mathcal{H}$.

We have $\mathbb{C} S\left(X, \frac{1}{k} \mathcal{M}\right)=\left\{P_{1}, P_{2}\right\}$. Now we can apply the proof of Lemma 21.3 to the proper transform of $\mathcal{M}$ on $U_{12}$ to get a contradiction. The claim of Proposition 21.1 is proved.

22. Case $n=29$, hypersurface of Degree 16 In $\mathbb{P}(1,1,2,5,8)$.

We use the notations and assumptions of Section 3 , Let $n=29$. Then $X$ is a sufficiently general hypersurface in $\mathbb{P}(1,1,2,5,8)$ of degree 16 , the equality $-K_{X}^{3}=1 / 5$ holds, and the singularities of the hypersurface $X$ consist of the points $O_{1}$ and $O_{2}$ that are quotient singularities of type $\frac{1}{2}(1,1,1)$, and the point $P$ that is a quotient singularity of type $\frac{1}{5}(1,2,3)$. 
The hypersurface $X$ is birationally superrigid, and there is a commutative diagram

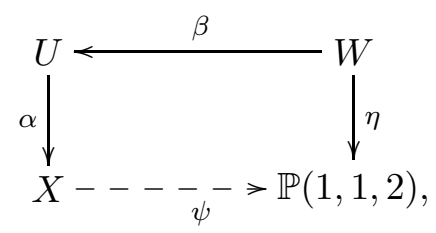

where $\psi$ is the natural projection, $\alpha$ is the weighted blow up of $P$ with weights $(1,2,3), \beta$ is the weighted blow up with weights $(1,1,2)$ of the singular point of the variety $U$ that is a quotient singularity of type $\frac{1}{3}(1,1,2)$, and $\eta$ is an elliptic fibration.

Proposition 22.1. The claim of Theorem 1.10 holds for $n=29$.

Proof. Let $\mathcal{D}$ be the proper transform of $\mathcal{M}$ on the variety $U$. Then $\mathcal{D} \sim_{\mathbb{Q}}-k K_{U}$ by Theorem 2.2 because $\mathbb{C S}\left(X, \frac{1}{k} \mathcal{M}\right)=\{P\}$ by Theorem 3.5. Lemma 3.14 and Proposition 3.7

Let $G$ is the $\alpha$-exceptional divisor, and $Q$ and $O$ be the singular points of $G$ that are singularities of types $\frac{1}{3}(1,1,2)$ and $\frac{1}{2}(1,1,1)$ respectively. Then it follows from Lemmas 2.1$]$ and 2.3 that either the set $\mathbb{C} \mathbb{S}\left(U, \frac{1}{k} \mathcal{D}\right)$ consists of the point $O$, or the set $\mathbb{C S}\left(U, \frac{1}{k} \mathcal{D}\right)$ contains $Q$.

Suppose that the set $\mathbb{C} \mathbb{S}\left(U, \frac{1}{k} \mathcal{D}\right)$ contains the point $O$. Let $\pi: Y \rightarrow U$ be the weighted blow up of $O$ with weights $(1,1,1), \mathcal{H}$ be the proper transform of $\mathcal{M}$ on $Y, L$ be the curve on $G$ that is contained in $\left|\mathcal{O}_{\mathbb{P}(1,2,3)}(1)\right|, \bar{L}$ be the proper transform of $L$ on $Y$, and $S$ be a general surface of the linear system $\left|-K_{Y}\right|$. Then $\mathcal{H} \sim_{\mathbb{Q}}-k K_{Y}$ by Theorem 2.2 and the base locus of the pencil $\left|-K_{Y}\right|$ consists of the curve $\bar{L}$ and the irreducible curve $\Delta$ such that $\alpha \circ \pi(\Delta)$ is the base locus of the pencil $\left|-K_{X}\right|$. Moreover, the equalities

$$
\Delta^{2}=-1, \bar{L}^{2}=-4 / 3, \Delta \cdot \bar{L}=1
$$

holds on the surface $S$. The intersection form of the curves $\Delta$ and $\bar{L}$ on the surface $S$ is negatively defined. We have $\left.\mathcal{H}\right|_{S} \sim_{\mathbb{Q}} k \Delta+k \bar{L}$, which contradicts Lemmas 2.8 and 2.10.

Therefore, the set $\mathbb{C S}\left(U, \frac{1}{k} \mathcal{D}\right)$ contains the point $Q$. Let $\mathcal{B}$ be the proper transform of the linear system $\mathcal{M}$ on the variety $W$. Then $\mathcal{B} \sim_{\mathbb{Q}}-k K_{W}$ by Theorem 2.2 , which implies that the linear system $\mathcal{B}$ is contained in the fibers of the morphism $\eta$.

\section{Case $n=30$, hypersurface of Degree 16 In $\mathbb{P}(1,1,3,4,8)$.}

We use the notations and assumptions of Section [3. Let $n=30$. Then $X$ is a sufficiently general hypersurface in $\mathbb{P}(1,1,3,4,8)$ of degree 16 , the equality $-K_{X}^{3}=1 / 6$ holds, and the singularities of $X$ consist of the point $O$ that is a quotient singularity of type $\frac{1}{3}(1,1,2)$, and the points $P_{1}$ and $P_{2}$ that are singularities of type $\frac{1}{4}(1,1,3)$. There is a commutative diagram

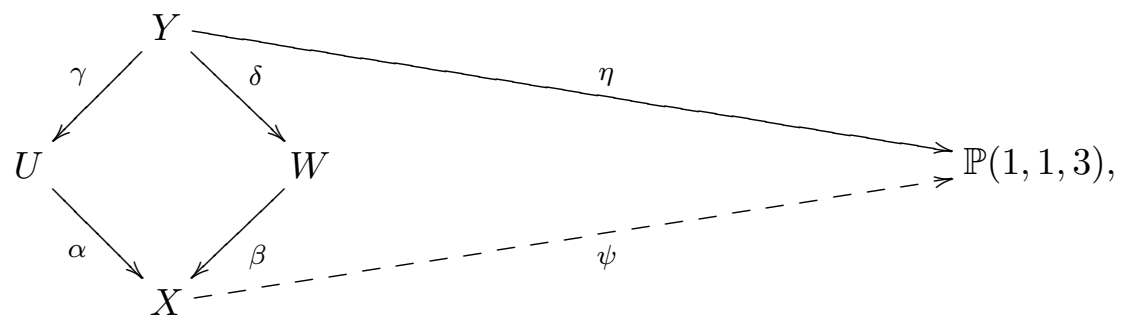

where $\psi$ is the natural projection, $\alpha$ is the weighted blow up of $P_{1}$ with weights $(1,1,3), \beta$ is the weighted blow up of $P_{2}$ with weights $(1,1,3), \gamma$ is the weighted blow up with weights $(1,1,3)$ of the proper transform of $P_{2}$ on $U, \delta$ is the weighted blow up with weights $(1,1,3)$ of the proper transform of the point $P_{1}$ on the variety $W$, and $\eta$ is and elliptic fibration.

There is a commutative diagram

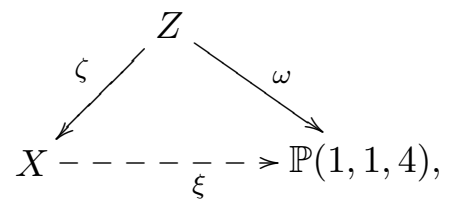

where $\xi$ is a projection, $\zeta$ is the blow up of $O$ with weights $(1,1,2)$, and $\omega$ is an elliptic fibration. 
Proposition 23.1. Either there is a commutative diagram

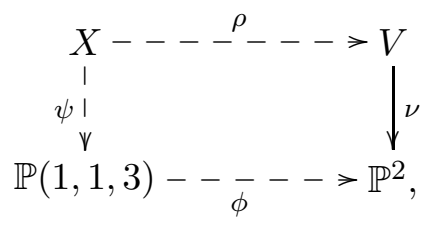

or there is a commutative diagram

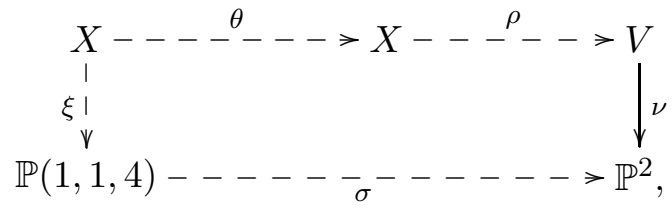

where $\phi, \theta$ and $\sigma$ are birational maps.

Proof. Suppose that $O \in \mathbb{C} \mathbb{S}\left(X, \frac{1}{k} \mathcal{M}\right)$. Then the existence of the commutative diagram 23.3 follows from Theorem 2.2. Similarly, the existence of the commutative diagram 23.2 follows from $\mathbb{C S}\left(X, \frac{1}{k} \mathcal{M}\right)=\left\{P_{1}, P_{2}\right\}$ by Theorem 2.2. We may assume that $\mathbb{C} \mathbb{S}\left(X, \frac{1}{k} \mathcal{M}\right)$ consists of the singular point $P_{1}$ by Theorem 3.5. Proposition 3.7 and Lemma 3.14.

Let $Q$ be the singular point of $U$ such that $\alpha(Q)=P_{1}$, and $\mathcal{B}$ be the proper transform of the linear system $\mathcal{M}$ on $U$. Then $Q \in \mathbb{C} S\left(U, \frac{1}{k} \mathcal{B}\right)$ by Theorem 2.2 and Lemmas 2.1] and 2.3

Let $v: \bar{U} \rightarrow U$ be the weighted blow up of the point $Q$ with weights $(1,1,2), \mathcal{D}$ be the proper transform of the linear system $\mathcal{M}$ on the variety $\bar{U}$, and $S$ be a sufficiently general surface of the pencil $\left|-K_{\bar{U}}\right|$. Then $\mathcal{D} \sim_{\mathbb{Q}}-k K_{\bar{U}}$ by Theorem 2.2 the surface $S$ is normal, and the base locus of the pencil $\left|-K_{\bar{U}}\right|$ consists of the irreducible curve $\Delta$ such that $\alpha \circ v(\Delta)$ is the unique base curve of the pencil $\left|-K_{X}\right|$. Moreover, the inequality $\Delta^{2}<0$ holds on the surface $S$, but the equivalence $\left.\mathcal{D}\right|_{S} \sim_{\mathbb{Q}} k \Delta$ holds, which contradicts Lemmas 2.10 and 2.8

24. Case $n=31$, hypersurface of Degree 16 In $\mathbb{P}(1,1,4,5,6)$.

We use the notations and assumptions of Section 3 Let $n=31$. Then $X$ is a general hypersurface in $\mathbb{P}(1,1,2,3,4)$ of degree 10 and $-K_{X}^{3}=2 / 15$. The singularities of $X$ consist of the points $P_{1}, P_{2}$ and $P_{3}$ that are singularities of type $\frac{1}{2}(1,1,1), \frac{1}{5}(1,1,4)$ and $\frac{1}{6}(1,1,5)$ respectively.

There is a commutative diagram

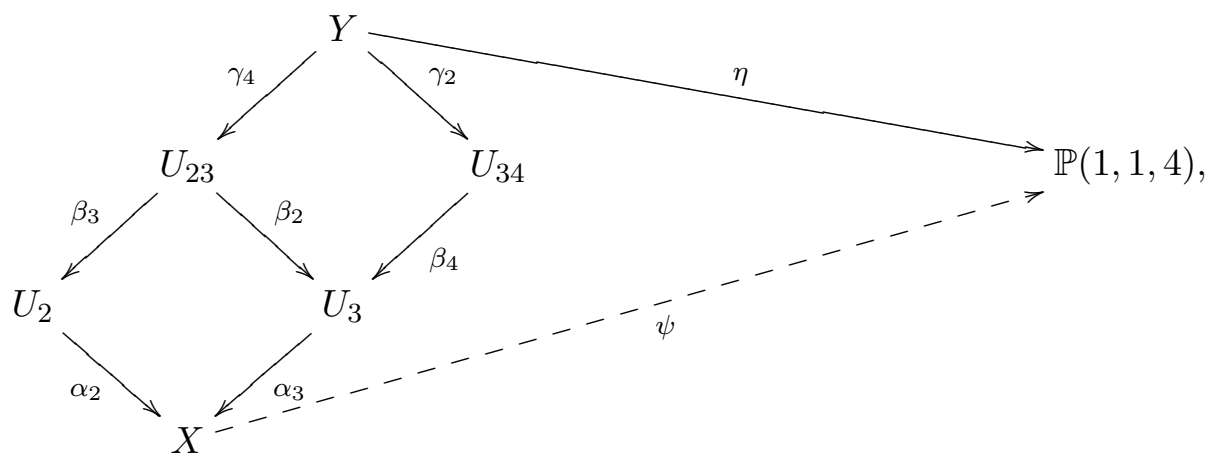

where $\psi$ is a projection, $\alpha_{2}$ is the weighted blow up of $P_{2}$ with weights $(1,1,4), \alpha_{3}$ is the weighted blow up of $P_{3}$ with weights $(1,1,5), \beta_{3}$ is the weighted blow up with weights $(1,1,5)$ of the proper transform of $P_{3}$ on $U_{2}, \beta_{2}$ is the weighted blow up with weights $(1,1,4)$ of the proper transform of the point $P_{2}$ on the variety $U_{3}, \beta_{4}$ is the weighted blow up with weights $(1,1,4)$ of the singular point of the variety $U_{3}$ that is contained in the exceptional divisor of the morphism $\alpha_{3}, \gamma_{2}$ is the weighted blow up with weights $(1,1,4)$ of the proper transform of $P_{2}$ on $U_{34}, \gamma_{4}$ is the weighted blow up with weights $(1,1,4)$ of the singular point of the variety $U_{23}$ that is contained in the 
exceptional divisor of $\beta_{3}$, and $\eta$ is an elliptic fibration. There is a commutative diagram

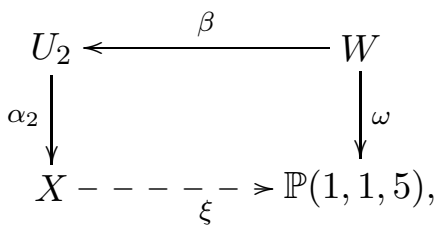

where $\xi$ is a projection, $\beta$ is the weighted blow up with weights $(1,1,3)$ of the singular point of the variety $U_{2}$ that is a quotient singularity of type $\frac{1}{4}(1,1,3)$, and $\omega$ is an elliptic fibration.

Proposition 24.1. Either there is a commutative diagram

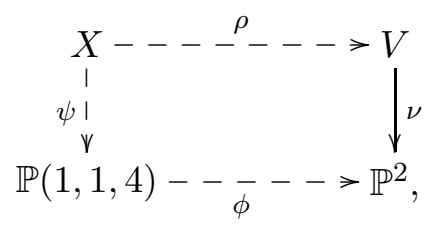

or there is a commutative diagram

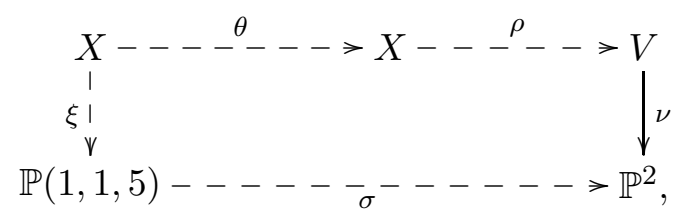

where $\phi, \theta$ and $\sigma$ are birational maps.

Now we prove Proposition 24.1, which implies the claim of Theorem 1.10 for $n=31$. It follows from Theorem 3.5. Lemma 3.14 and Proposition 3.7 that

$$
\varnothing \neq \mathbb{C} S\left(X, \frac{1}{k} \mathcal{M}\right) \subseteq\left\{P_{2}, P_{3}\right\} .
$$

Let $\mathcal{D}_{2}, \mathcal{D}_{3}, \mathcal{D}_{23}$ and $\mathcal{D}_{34}$ be the proper transforms of $\mathcal{M}$ on $U_{2}, U_{3}, U_{23}$ and $U_{34}$ respectively, then it follows from Lemma 2.1 that the set $\mathbb{C} S\left(U_{\mu}, \frac{1}{k} \mathcal{D}_{\mu}\right)$ is not empty, if $\mathcal{D}_{\mu} \sim_{\mathbb{Q}}-k K_{U_{\mu}}$.

Lemma 24.4. Suppose that the set $\mathbb{C S}\left(X, \frac{1}{k} \mathcal{M}\right)$ contains the point $P_{3}$. Let $\bar{P}_{2}$ be the proper transform of the point $P_{2}$ on the variety $U_{3}$, and $\bar{P}_{4}$ be the singular point of $U_{3}$ that is contained in the exceptional divisor of $\alpha_{3}$. Then $\mathcal{D}_{3} \sim_{\mathbb{Q}}-k K_{U_{3}}$ and $\mathbb{C S}\left(U_{3}, \frac{1}{k} \mathcal{D}_{3}\right) \subseteq\left\{\bar{P}_{2}, \bar{P}_{4}\right\}$.

Proof. The equivalence $\mathcal{D}_{3} \sim_{\mathbb{Q}}-k K_{U_{3}}$ follows from Theorem 2.2. Suppose that

$$
\mathbb{C S}\left(U_{3}, \frac{1}{k} \mathcal{D}_{3}\right) \nsubseteq\left\{\bar{P}_{2}, \bar{P}_{4}\right\}
$$

and let $G$ be the exceptional divisor of $\alpha_{3}$. Then $G \cong \mathbb{P}(1,1,5)$, and it follows from Lemma 2.3 that there is a curve $C \subset G$ of the linear system $\left|\mathcal{O}_{\mathbb{P}(1,1,5)}(1)\right|$ that is contained in $\mathbb{C S}\left(U_{3}, \frac{1}{k} \mathcal{D}_{3}\right)$, which is impossible by Lemma 2.4 .

Lemma 24.5. Let $\bar{P}_{3}$ be the proper transform of $P_{3}$ on $U_{2}$. Suppose that $\mathbb{C S}\left(X, \frac{1}{k} \mathcal{M}\right)$ contains the point $P_{2}$. Then either $\mathbb{C S}\left(U_{2}, \frac{1}{k} \mathcal{D}_{2}\right)=\left\{\bar{P}_{3}\right\}$, or the commutative diagram 24.3 exists.

Proof. The equivalence $\mathcal{D}_{2} \sim_{\mathbb{Q}}-k K_{U_{2}}$ is implied by Theorem 2.2. Suppose that the set of centers of canonical singularities $\mathbb{C S}\left(U_{2}, \frac{1}{k} \mathcal{D}_{2}\right)$ does not consists of the point $\bar{P}_{3}$. Let $\bar{P}_{5}$ be the singular point of $U_{2}$ that is contained in the exceptional divisor of $\alpha_{2}$. Then $\bar{P}_{5}$ is a quotient singularity of type $\frac{1}{4}(1,1,3)$ on $U_{2}$, and $\mathbb{C S}\left(U_{2}, \frac{1}{k} \mathcal{D}_{2}\right)$ contains $\bar{P}_{5}$ by Lemma 2.3 .

Let $\mathcal{B}$ be the proper transform of $\mathcal{M}$ on the variety $W$. Then $\mathcal{B} \sim_{\mathbb{Q}}-k K_{W}$ by Theorem 2.2 which implies the existence of the commutative diagram 24.3 .

It follows from Theorem 2.2 that we may assume that either the equivalence $\mathcal{D}_{23} \sim_{\mathbb{Q}}-k K_{U_{23}}$ holds, or the equivalence $\mathcal{D}_{34} \sim_{\mathbb{Q}}-k K_{U_{34}}$ holds. Let $\mathcal{D}$ be the proper transform of $\mathcal{M}$ on $Y$.

Lemma 24.6. Suppose that $\mathcal{D}_{23} \sim_{\mathbb{Q}}-k K_{U_{23}}$. Then $\mathcal{D} \sim_{\mathbb{Q}}-k K_{Y}$. 
Proof. Let $F$ be the exceptional divisor of $\beta_{2}, G$ be the exceptional divisor of $\beta_{3}, \check{P}_{4}$ be the singular point of $G$, and $\dot{P}_{5}$ be the singular point of $F$. Then the proof of Lemma 24.5 implies that $\mathbb{C S}\left(U_{23}, \frac{1}{k} \mathcal{D}_{23}\right)$ does not contain the point $\check{P}_{5}$. Hence, it follows from Lemmas 2.3 and 2.1 that the set $\mathbb{C} S\left(U_{23}, \frac{1}{k} \mathcal{D}_{23}\right)$ contains $\check{P}_{4}$, which implies $\mathcal{D} \sim_{\mathbb{Q}}-k K_{Y}$ by Theorem 2.2 .

Lemma 24.7. Suppose that $\mathcal{D}_{34} \sim_{\mathbb{Q}}-k K_{U_{34}}$. Then $\mathcal{D} \sim_{\mathbb{Q}}-k K_{Y}$.

Proof. Let $G$ be the exceptional divisor of the morphism $\beta_{4}, \check{P}_{2}$ be the proper transform of the point $P_{2}$ on the variety $U_{34}$, and $\check{P}_{6}$ be the singular point of the surface $G$. Then $G$ is a cone over the smooth rational cubic curve, and $\check{P}_{6}$ is a quotient singularity of type $\frac{1}{3}(1,1,2)$ on $U_{34}$.

The set $\mathbb{C} \mathbb{S}\left(U_{23}, \frac{1}{k} \mathcal{D}_{23}\right)$ is not empty by Lemma 2.1 but the equivalence $\mathcal{D} \sim_{\mathbb{Q}}-k K_{Y}$ follows from Theorem 2.2 if the set $\mathbb{C S}\left(U_{23}, \frac{1}{k} \mathcal{D}_{23}\right)$ contains the point $\check{P}_{2}$. Therefore, we may assume that the set $\mathbb{C} S\left(U_{23}, \frac{1}{k} \mathcal{D}_{23}\right)$ contains the point $\check{P}_{6}$ by Lemma 2.3 .

Let $\pi: W \rightarrow U_{34}$ be the weighted blow up of the point $\check{P}_{6}$ with weights $(1,1,3), \mathcal{B}$ be the proper transform of the linear system $\mathcal{M}$ on the variety $W$, and $S$ be a sufficiently general surface of the pencil $\left|-K_{W}\right|$. Then the base locus of the pencil $\left|-K_{W}\right|$ consists of the irreducible the curve $\Delta$ such that the equivalence $\left.\mathcal{B}\right|_{S} \sim_{\mathbb{Q}} k \Delta$ holds, and the inequality $\Delta^{2}<0$ holds on the surface $S$. It follows from Lemma 2.10 that $\left.\mathcal{B}\right|_{S}=k \Delta$, which is impossible by Lemma 2.8

Hence, the equivalence $\mathcal{D} \sim_{\mathbb{Q}}-k K_{Y}$ holds, which implies the existence of the diagram 24.2

\section{CASE $n=32$, hypersurface OF DEgree 16 IN $\mathbb{P}(1,2,3,4,7)$.}

We use the notations and assumptions of Section [3. Let $n=32$. Then $X$ is a sufficiently general hypersurface in $\mathbb{P}(1,2,3,4,6)$ of degree 16 , the equality $-K_{X}^{3}=2 / 21$ holds, and the singularities of the hypersurface $X$ consist of the points $P_{1}, P_{2}, P_{3}$ and $P_{4}$ that are quotient singularities of type $\frac{1}{2}(1,1,1)$, the point $P_{5}$ that is a quotient singularity of type $\frac{1}{3}(1,1,2)$, and the point $P_{6}$ that is a singularity of type $\frac{1}{7}(1,3,4)$. There is a commutative diagram

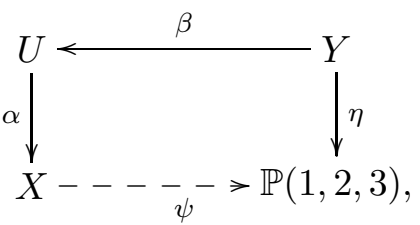

where $\psi$ is a projection, $\alpha$ is the weighted blow up of $P_{6}$ with weights $(1,3,4), \beta$ is the weighted blow up with weights $(1,1,3)$ of the singular point of the variety $U$ that is a quotient singularity of type $\frac{1}{4}(1,1,3)$ contained in the exceptional divisor of $\alpha$, and $\eta$ is an elliptic fibration.

Proposition 25.1. The claim of Theorem 1.10 holds for $n=32$.

In the rest of the section we prove Proposition 25.1] It follows from Theorem 3.5 Lemma 3.14 and Proposition 3.7 that $\mathbb{C S}\left(X, \frac{1}{k} \mathcal{M}\right)=\left\{P_{6}\right\}$.

Let $E$ be the exceptional divisor of the morphism $\alpha$, and $\mathcal{D}$ be the proper transform of the linear system $\mathcal{M}$ on the variety $U$. Then $E \cong \mathbb{P}(1,3,4)$, and it follows from Theorem 2.2 that the equivalence $\mathcal{D} \sim_{\mathbb{Q}}-k K_{U}$ holds, but the set $\mathbb{C S}\left(U, \frac{1}{k} \mathcal{D}\right)$ is not empty by Lemma 2.1.

Let $P_{7}$ and $P_{8}$ be the singular points of the variety $U$ contained in the divisor $E$ that are singularities of types $\frac{1}{3}(1,1,2)$ and $\frac{1}{4}(1,1,3)$ respecitvely.

Lemma 25.2. Suppose that $P_{8} \in \mathbb{C S}\left(X, \frac{1}{k} \mathcal{M}\right)$. Then there is a commutative diagram

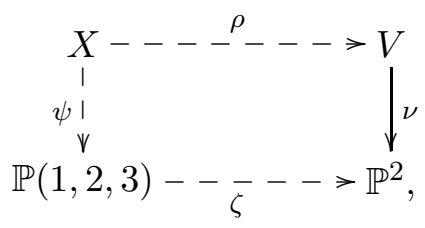

where $\zeta$ is a birational map. 
Proof. Let $\mathcal{H}$ be the proper transform of the linear system $\mathcal{M}$ on the variety $Y$. Then it follows from Theorem 2.2 that the equivalence $\mathcal{H} \sim_{\mathbb{Q}}-k K_{Y}$ holds. Hence, the linear system $\mathcal{H}$ lies in the fibers of the fibration $\eta$, which implies the existence of the commutative diagram 25.3 .

We may assume that $\mathbb{C} \mathbb{S}\left(U, \frac{1}{k} \mathcal{D}\right)=\left\{P_{7}\right\}$ by Lemma 2.3 .

Let $\gamma: W \rightarrow U$ be the weighted blow up of the point $P_{7}$ with weights $(1,1,2), F$ be the exceptional divisor of the morphism $\gamma, \bar{E}$ be the proper transform of the surface $E$ on the variety $W$, and $\mathcal{B}$ be the proper transform of $\mathcal{M}$ on $W$. Then $F \cong \mathbb{P}(1,1,2)$ and $\mathcal{B} \sim_{\mathbb{Q}}-k K_{W}$.

The hypersurface $X$ can be given by the quasihomogeneous equation

$$
w^{2} y+w f_{9}(x, y, z, t)+f_{16}(x, y, z, t)=0 \subset \mathbb{P}(1,2,3,4,7) \cong \operatorname{Proj}(\mathbb{C}[x, y, z, t, w]),
$$

where $\operatorname{wt}(x)=1, \operatorname{wt}(y)=2, \operatorname{wt}(z)=3, \operatorname{wt}(t)=4, \operatorname{wt}(w)=7$, and $f_{9}$ and $f_{16}$ are quasihomogeneous polynomials of degree 9 and 16 respectively. Let $S$ be the unique surface of the linear system $\left|-K_{X}\right|$, and $D$ be a general surface of the pencil $\left|-2 K_{X}\right|$. Then the surface $S$ is cut out by $x=0$, and $D$ is cut out by $\lambda x^{2}+\mu y=0$, where $(\lambda, \mu) \in \mathbb{P}^{1}$. The surface $D$ is normal, and the base locus of the linear system $\left|-2 K_{X}\right|$ consists of the curve $C$ such that $C=D \cdot S$.

In the neighborhood of the point $P_{6}$, the monomials $x, z$ and $t$ can be considered as a weighted local coordinates on $X$ such that $\operatorname{wt}(x)=1, \operatorname{wt}(z)=3$ and $\operatorname{wt}(z)=4$. Then in the neighborhood of the singular point $P_{6}$, the surface $D$ can be given by equation

$$
\lambda x^{2}+\mu\left(\epsilon_{1} x^{9}+\epsilon_{2} z x^{6}+\epsilon_{3} z^{2} x^{3}+\epsilon_{4} z^{3}+\epsilon_{5} t^{2} x+\epsilon_{6} t x^{5}+\epsilon_{7} t z x^{2}+\cdots\right)=0,
$$

where $\epsilon_{i} \in \mathbb{C}$. In the neighborhood of $P_{7}$, the morphism $\alpha$ can be given by the equations

$$
x=\tilde{x} \tilde{z}^{\frac{1}{7}}, z=\tilde{z}^{\frac{3}{7}}, t=\tilde{t} \tilde{z}^{\frac{4}{7}},
$$

where $\tilde{x}, \tilde{y}$ and $\tilde{z}$ are weighted local coordinates on the variety $U$ in the neighborhood of the singular point $P_{7}$ such that $\operatorname{wt}(\tilde{x})=1, \operatorname{wt}(\tilde{z})=2$ and $\operatorname{wt}(\tilde{t})=1$. Let $\tilde{D}, \tilde{S}$ and $\tilde{C}$ be the proper transforms on $U$ of the surface $D$, the surface $S$ and the curve $C$ respectively, and $E$ be the exceptional divisor of the morphism $\alpha$. Then in the neighborhood of the singular point $P_{7}$ the surface $E$ is given by the equation $\tilde{z}=0$, the surface $\tilde{D}$ is given by the vanishing of the function

$$
\lambda \tilde{x}^{2}+\mu\left(\epsilon_{1} \tilde{x}^{9} \tilde{z}+\epsilon_{2} \tilde{z} \tilde{x}^{6}+\epsilon_{3} \tilde{z} \tilde{x}^{3}+\epsilon_{4} \tilde{z}+\epsilon_{5} \tilde{t}^{2} \tilde{x} \tilde{z}+\epsilon_{6} \tilde{t} \tilde{x}^{5} \tilde{z}+\epsilon_{7} \tilde{t} \tilde{z} \tilde{x}^{2}+\cdots\right),
$$

and the surface $S$ is given by the equation $\tilde{x}=0$.

In the neighborhood of the singular point of $F$, the morphism $\beta$ can be given by the equations

$$
\tilde{x}=\bar{x} \bar{z}^{\frac{1}{3}}, \tilde{z}=\bar{z}^{\frac{2}{3}}, \tilde{t}=\bar{t} \bar{z}^{\frac{1}{3}}
$$

where $\bar{x}, \bar{z}$ and $\bar{t}$ are weighted local coordinates on the variety $W$ in the neighborhood of the singular point of the surface $F$ such that $\operatorname{wt}(\bar{x})=\operatorname{wt}(\bar{z})=\operatorname{wt}(\bar{t})=1$. The surface $F$ is given by the equation $\bar{z}=0$, the proper transform of the surface $D$ on the variety $W$ is given by the vanishing of the analytical function

$$
\lambda \bar{x}^{2}+\mu\left(\epsilon_{1} \bar{x}^{9} \bar{z}^{3}+\epsilon_{2} \bar{z}^{2} \bar{x}^{6}+\epsilon_{3} \bar{z} \bar{x}^{3}+\epsilon_{4}+\epsilon_{5} \bar{t}^{2} \bar{x} \bar{z}+\epsilon_{6} \bar{t} \bar{x}^{5} \bar{z}^{2}+\epsilon_{7} \bar{t} \bar{z} \bar{x}^{2}+\cdots\right),
$$

the proper transform of the surface $S$ on the variety $W$ is given by the equation $\bar{x}=0$, and the proper transform of the surface $E$ of the variety $W$ is given by the equation $\bar{z}=0$.

Let $\mathcal{P}, \bar{D}, \bar{S}$ and $\bar{C}$ be the proper transforms on the variety $W$ of the pencil $\left|-2 K_{X}\right|$, the surface $D$, the surface $S$ and the curve $C$ respectively, and $\bar{H}$ be the proper transform on the variety $W$ of the surface that is cut on $X$ by the equation $y=0$. Then the surface $\bar{D}$ is a general surface of the pencil $\mathcal{P}$. Moreover, we have

$$
\left\{\begin{array}{l}
\bar{E} \sim_{\mathbb{Q}} \gamma^{*}(E)-\frac{2}{3} F \\
\bar{D} \sim_{\mathbb{Q}}(\alpha \circ \gamma)^{*}\left(-2 K_{X}\right)-\frac{2}{7} \gamma^{*}(E)-\frac{2}{3} F \sim_{\mathbb{Q}}(\alpha \circ \gamma)^{*}\left(-2 K_{X}\right)-\frac{2}{7} \bar{E}-\frac{6}{7} F, \\
\bar{S} \sim_{\mathbb{Q}}(\alpha \circ \gamma)^{*}\left(-K_{X}\right)-\frac{1}{7} \gamma^{*}(E)-\frac{1}{3} F \sim_{\mathbb{Q}}(\alpha \circ \gamma)^{*}\left(-K_{X}\right)-\frac{1}{7} \bar{E}-\frac{3}{7} F \\
\bar{H} \sim_{\mathbb{Q}} \gamma^{*}\left(\alpha^{*}\left(-2 K_{X}\right)-\frac{9}{7} E\right) \sim_{\mathbb{Q}}(\alpha \circ \gamma)^{*}\left(-2 K_{X}\right)-\frac{9}{7} \bar{E}-\frac{6}{7} F \sim_{\mathbb{Q}} 2 \bar{S}-\bar{E}
\end{array}\right.
$$


The curve $\bar{C}$ is contained in the base locus of the pencil $\mathcal{P}$, but the curve $\bar{C}$ is not the only curve in the base locus of the pencil $\mathcal{P}$. Namely, let $L$ be the curve on the surface $E$ that is contained in the linear system $\left|\mathcal{O}_{\mathbb{P}(1,3,4)}(1)\right|$, which means that $L$ is the curve given locally by the equations $\tilde{x}=\tilde{z}=0$, and $\bar{L}$ be the proper transform of $L$ on $W$. Then the curve $\bar{L}$ is contained in the base locus of the pencil $\mathcal{P}$ as well. Moreover, it follows from the local computations that the base locus of the pencil $\mathcal{P}$ does not contain curves outside of the union of $\bar{C} \cup \bar{L}$.

The curve $\bar{C}$ is the intersection of the divisors $\bar{S}$ and $\bar{H}$, and the curve $\bar{L}$ is the intersections of the divisors $\bar{S}$ and $\bar{E}$. Moreover, we have $2 \bar{C}=\bar{D} \cdot \bar{H}, \bar{C}+\bar{L}=\bar{S} \cdot \bar{D}$ and $2 \bar{L}=\bar{D} \cdot \bar{E}$.

The curves $\bar{C}$ and $\bar{L}$ can be considered as divisors on the normal surface $\bar{D}$. Then it follows from the equivalences 25.4 that

$$
\left\{\begin{array}{l}
\bar{L} \cdot \bar{L}=\frac{\bar{E} \cdot \bar{E} \cdot \bar{D}}{4}=-\frac{5}{8} \\
\bar{C} \cdot \bar{C}=\frac{\bar{H} \cdot \bar{H} \cdot \bar{D}}{4}=\bar{S} \cdot \bar{S} \cdot \bar{D}-\bar{S} \cdot \bar{E} \cdot \bar{D}-\frac{3}{2} \bar{E} \cdot \bar{E} \cdot \bar{D}=-\frac{7}{24}, \\
\bar{C} \cdot \bar{L}=\frac{\bar{H} \cdot \bar{E} \cdot \bar{D}}{4}=\frac{\bar{S} \cdot \bar{E} \cdot \bar{D}}{2}-\frac{\bar{E} \cdot \bar{E} \cdot \bar{D}}{4}=\frac{3}{8},
\end{array}\right.
$$

which implies that the intersection forms of $\bar{C}$ and $\bar{L}$ on $\bar{D}$ is negatively definite.

Let $G$ be a sufficiently general surface of the linear system $\mathcal{B}$. Then

$$
\left.G\right|_{\bar{D}} \sim_{\mathbb{Q}}-\left.\left.k K_{W}\right|_{\bar{D}} \sim_{\mathbb{Q}} k \bar{S}\right|_{\bar{D}} \sim_{\mathbb{Q}} k \bar{C}+k \bar{L}
$$

which is impossible by Lemmas 2.8 and 2.10 .

\section{Case $n=36$, hypersurface of Degree 18 in $\mathbb{P}(1,1,4,6,7)$.}

We use the notations and assumptions of Section 3 . Let $n=36$. Then $X$ is a sufficiently general hypersurface in $\mathbb{P}(1,1,4,6,7)$ of degree 18 , the equality $-K_{X}^{3}=3 / 28$ holds, and the singularities of $X$ consist of the point $P_{1}$ that is a quotient singularity of type $\frac{1}{2}(1,1,1)$, the point $P_{2}$ that is a quotient singularity of type $\frac{1}{4}(1,1,3)$, and the point $P_{3}$ that is a quotient singularity of type $\frac{1}{7}(1,1,6)$. There is a commutative diagram

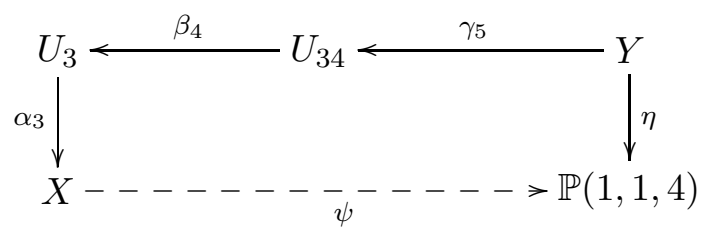

where $\psi$ is a projection, $\alpha_{3}$ is the weighted blow up of $P_{3}$ with weights $(1,1,7), \beta_{4}$ is the weighted blow up with weights $(1,1,6)$ of the singular point of the variety $U_{3}$ that is contained in the exceptional divisor of the morphism $\alpha_{3}, \gamma_{5}$ is the weighted blow up with weights $(1,1,4)$ of the singular point of the variety $U_{34}$ that is contained in the exceptional divisor of the birational morphism $\beta_{4}$, and $\eta$ is an elliptic fibration.

Remark 26.1. The divisors $-K_{U_{3}}$ and $-K_{U_{34}}$ are nef and big.

There is a commutative diagram

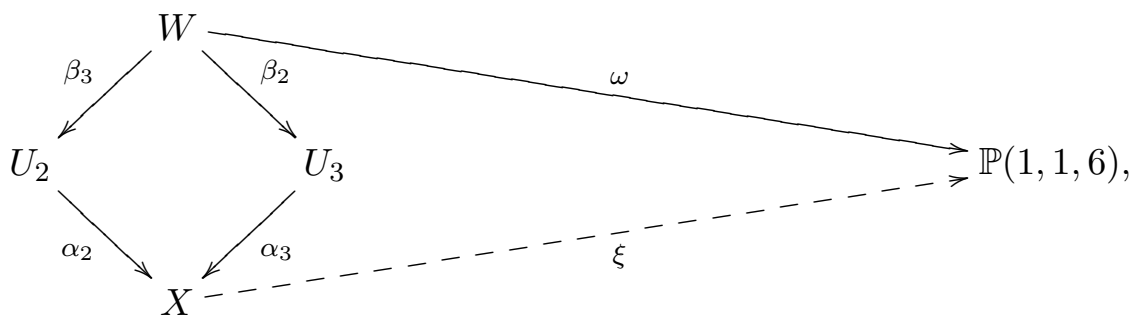

where $\xi$ is a projection, $\alpha_{2}$ is the blow up of the $P_{2}$ with weights $(1,1,3), \alpha_{3}$ is the weighted blow up of $P_{3}$ with weights $(1,1,6), \beta_{2}$ is the weighted blow up with weights $(1,1,3)$ of the proper transform of $P_{2}$ on $U_{3}, \beta_{3}$ is the weighted blow up with weights $(1,1,6)$ of the proper transform of the point $P_{3}$ on $U_{2}$, and $\omega$ is an elliptic fibration. 
Remark 26.2. The divisor $-K_{U_{2}}$ is nef and big.

In the rest of the section we prove the following result.

Proposition 26.3. Either there is a commutative diagram

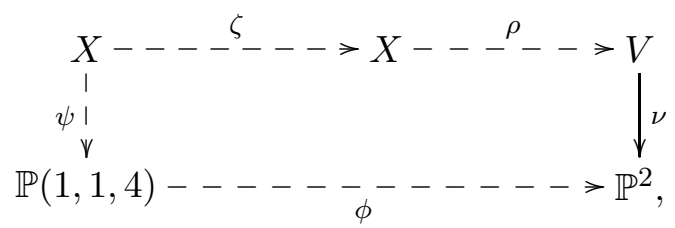

or there is a commutative diagram

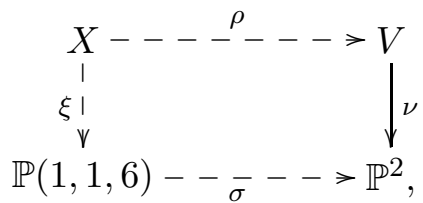

where $\zeta, \phi$ and $\sigma$ are birational maps.

It follows from Theorem 3.5. Proposition 3.7 and Lemma 3.14 that

$$
\varnothing \neq \mathbb{C} S\left(X, \frac{1}{k} \mathcal{M}\right) \subseteq\left\{P_{2}, P_{3}\right\},
$$

and the existence of the commutative diagram 20.3 is obvious, if $\mathbb{C} S\left(X, \frac{1}{k} \mathcal{M}\right)=\left\{P_{2}, P_{3}\right\}$.

Lemma 26.6. The set $\mathbb{C} \mathbb{S}\left(X, \frac{1}{k} \mathcal{M}\right)$ contains point $P_{3}$.

Proof. Suppose that $P_{3} \notin \mathbb{C S}\left(X, \frac{1}{k} \mathcal{M}\right)$. Then $\mathbb{C} S\left(X, \frac{1}{k} \mathcal{M}\right)=\left\{P_{2}\right\}$. Let $\mathcal{D}_{2}$ be the proper transform of $\mathcal{M}$ on $U_{2}$, and $P_{6}$ be the singular point of $U_{2}$ that is contained in the exceptional divisor of $\alpha_{2}$. Then $\mathcal{D}_{2} \sim_{\mathbb{Q}}-k K_{U_{2}}$ by Theorem 2.2, the point $P_{6}$ is a quotient singularity of type $\frac{1}{3}(1,1,2)$ on $U_{2}$, and it follows from Lemmas 2.1 and 2.3 that $P_{6} \in \mathbb{C} \mathbb{S}\left(U_{2}, \frac{1}{k} \mathcal{D}_{2}\right)$.

Let $\pi: Z \rightarrow U_{2}$ be the weighted blow up of $P_{6}$ with weights $(1,1,3)$, and $\mathcal{B}$ be the proper transform of $\mathcal{M}$ on $Z$, and $S$ be a general surface of $\left|-K_{Z}\right|$. Then $S$ is normal, and the base locus of the $\left|-K_{Z}\right|$ consists of the irreducible curve $\Delta$ such that $\left.\mathcal{B}\right|_{S} \sim_{\mathbb{Q}} k \Delta$.

The equality $\Delta^{2}=1 / 7$ holds on the surface $S$, which contradicts Lemmas 2.8 and 2.10 .

To conclude the proof of the Proposition 26.3, we may assume that $\mathbb{C S}\left(X, \frac{1}{k} \mathcal{M}\right)=\left\{P_{3}\right\}$.

Let $\mathcal{D}_{3}$ be the proper transform of $\mathcal{M}$ on $U_{3}$, and $P_{4}$ be the singular point of $U_{3}$ that is contained in the exceptional divisor of the $\alpha_{3}$. Then $\mathcal{D}_{3} \sim_{\mathbb{Q}}-k K_{U_{3}}$, and $P_{4}$ is a quotient singularity of type $\frac{1}{6}(1,1,5)$ on $U_{3}$ that is contained in $\mathbb{C} S\left(U_{3}, \frac{1}{k} \mathcal{D}_{3}\right)$ by Lemmas 2.1 and 2.3.

Lemma 26.7. The set $\mathbb{C} S\left(U_{3}, \frac{1}{k} \mathcal{D}_{3}\right)$ consists of the point $P_{4}$.

Proof. Suppose that the set $\mathbb{C S}\left(U_{3}, \frac{1}{k} \mathcal{D}_{3}\right)$ contains a subvariety $C$ of the variety $U_{3}$ that is different from the point $P_{4}$. Let $G$ be the exceptional divisor of $\beta_{4}$. Then $C$ is a curve that is contained in $G$ by Lemma 2.3 , which is impossible by Lemma 2.4 .

Hence, the set $\mathbb{C S}\left(U_{3}, \frac{1}{k} \mathcal{D}_{3}\right)$ consists of the point $P_{4}$. Let $\mathcal{D}_{34}$ be the proper transform of the linear system $\mathcal{M}$ on the variety $U_{34}$, and $P_{5}$ be the singular point of the variety $U_{34}$ that is contained in exceptional divisor of the morphism $\beta_{4}$. Then $\mathcal{D}_{34} \sim_{\mathbb{Q}}-k K_{U_{34}}$ by Theorem 2.2 the point $P_{5}$ is a quotient singularity of type $\frac{1}{5}(1,1,4)$ on $U_{34}$, but $\mathbb{C} \mathbb{S}\left(U_{34}, \frac{1}{k} \mathcal{D}_{34}\right)$ contains the point $P_{5}$ by Lemmas 2.1 and 2.3 It follows from Theorem 2.2 that the proper transform of the linear system $\mathcal{M}$ on $Y$ is contained in fibers of the elliptic fibration $\eta$, which implies the existence of the commutative diagram 26.4. The claim of Proposition 26.3] is proved. 
27. Case $n=38$, hypersurface of Degree 18 In $\mathbb{P}(1,2,3,5,8)$.

We use the notations and assumptions of Section 3 Let $n=38$. Then $X$ is a hypersurface of degree 18 in $\mathbb{P}(1,2,3,5,8)$, the singularities of $X$ consist of the points $P_{1}$ and $P_{2}$ that are quotient singularities of type $\frac{1}{2}(1,1,1)$, the point $P_{3}$ that is a quotient singularity of type $\frac{1}{5}(1,2,3)$, and the point $P_{4}$ that is a quotient singularity of type $\frac{1}{8}(1,3,5)$, and the equality $-K_{X}^{3}=3 / 40$ holds.

There is a commutative diagram

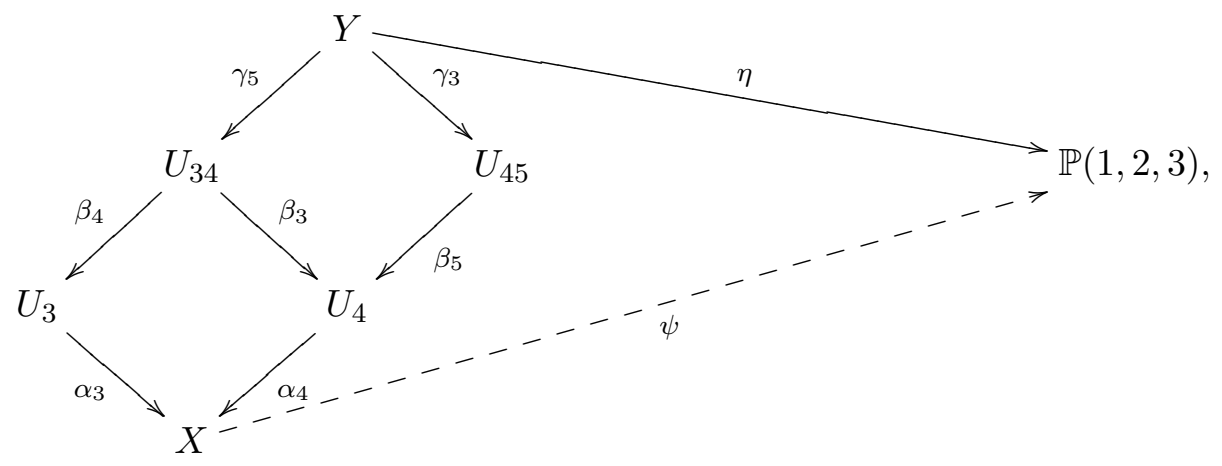

where $\psi$ is a projection, $\alpha_{3}$ is the weighted blow up of $P_{3}$ with weights $(1,2,3), \alpha_{4}$ is the weighted blow up of $P_{4}$ with weights $(1,3,5), \beta_{4}$ is the weighted blow up with weights $(1,3,5)$ of the proper transform of $P_{4}$ on $U_{3}, \beta_{3}$ is the weighted blow up with weights $(1,2,3)$ of the proper transform of the point $P_{3}$ on $U_{4}, \beta_{5}$ is the weighted blow up with weights $(1,2,3)$ of the singular point of the variety $U_{4}$ that is a quotient singularity of type $\frac{1}{5}(1,2,3)$ contained in the exceptional divisor of the morphism $\alpha_{4}, \gamma_{3}$ is the weighted blow up with weights $(1,2,3)$ of the proper transform of the point $P_{3}$ on the $U_{45}, \gamma_{5}$ is the weighted blow up with weights $(1,2,3)$ of the singular point of the variety $U_{34}$ that is a quotient singularity of type $\frac{1}{5}(1,2,3)$ contained in the exceptional divisor of the morphism $\beta_{4}$, and $\eta$ is an elliptic fibration.

Proposition 27.1. The claim of Theorem 1.10 holds for $n=38$.

Proof. It follows from Theorem 3.5. Lemma 3.14 and Proposition 3.7 that

$$
\varnothing \neq \mathbb{C S}\left(X, \frac{1}{k} \mathcal{M}\right) \subseteq\left\{P_{3}, P_{4}\right\},
$$

but the proof of Proposition 21.1 implies that $P_{4} \in \mathbb{C} S\left(X, \frac{1}{k} \mathcal{M}\right)$.

Let $\mathcal{D}_{4}$ be the proper transform of the linear system $\mathcal{M}$ on the variety $U_{4}, \bar{P}_{3}$ be the proper transform of the point $P_{3}$ on $U_{4}$, and $P_{5}$ and $P_{6}$ be the singular points of the variety $U_{4}$ that are quotient singularities of types $\frac{1}{5}(1,2,3)$ and $\frac{1}{3}(1,1,2)$ contained in exceptional divisor of the morphism $\alpha_{4}$ respectively. Then the arguments of the proof of Proposition 25.1 imply that

$$
\mathbb{C S}\left(U_{4}, \frac{1}{k} \mathcal{D}_{4}\right) \cap\left\{\bar{P}_{3}, P_{5}\right\} \neq \varnothing .
$$

Suppose that $\bar{P}_{3} \in \mathbb{C S}\left(U_{4}, \frac{1}{k} \mathcal{D}_{5}\right)$. Then the proofs of Propositions 21.1 and 25.1 implies that the set $\mathbb{C} S\left(U_{4}, \frac{1}{k} \mathcal{D}_{5}\right)$ contains the singular point $P_{5}$. Therefore, the claim of Theorem 2.2 implies that the claim of Theorem 1.10 holds for the hypersurface $X$.

We may assume that the set $\mathbb{C} S\left(U_{4}, \frac{1}{k} \mathcal{D}_{5}\right)$ contains the point $P_{5}$.

Let $\mathcal{D}_{45}$ be the proper transform of $\mathcal{M}$ on $U_{45}$, and $P_{7}$ and $P_{8}$ be the singular points of the variety $U_{45}$ that are quotient singularities of types $\frac{1}{2}(1,1,1)$ and $\frac{1}{3}(1,1,2)$ contained in the exceptional divisor of $\beta_{5}$ respectively. Then if follows from Lemma 2.1 that $\mathbb{C S}\left(U_{45}, \frac{1}{k} \mathcal{D}_{45}\right) \neq \varnothing$, and Theorem 2.2 implies that the claim of Theorem 1.10 holds for the hypersurface $X$ in the case when the set $\mathbb{C} \mathbb{S}\left(U_{45}, \frac{1}{k} \mathcal{D}_{45}\right)$ contains the proper transform of the point $P_{3}$ on $U_{45}$.

Therefore, it follows from Lemma 2.3] that to conclude the proof of Proposition 27.1] we may assume that the set $\mathbb{C S}\left(U_{45}, \frac{1}{k} \mathcal{D}_{45}\right)$ contains either the point $P_{7}$, or the point $P_{8}$.

Suppose that $P_{7} \in \mathbb{C} S\left(U_{45}, \frac{1}{k} \mathcal{D}_{45}\right)$. Then considering the proper transform of the complete linear system $\left|-3 K_{X}\right|$ on the weighted blow up of the point $P_{7}$ with weights $(1,1,1)$, we easily obtain a contradiction as in the proof of Lemma 21.3.

Thus, the set $\mathbb{C S}\left(U_{45}, \frac{1}{k} \mathcal{D}_{45}\right)$ contains the point $P_{8}$. 
Let $\pi: W \rightarrow U_{45}$ be the weighted blow up of $P_{8}, \mathcal{B}$ be the proper transform of $\mathcal{M}$ on the variety $W$, and $D$ be a general surface in $\left|-2 K_{W}\right|$. Then $\mathcal{B} \sim_{\mathbb{Q}}-k K_{W}$ by Theorem 2.2, the surface $D$ is normal, the pencil $\left|-2 K_{W}\right|$ is the proper transform of the pencil $\left|-2 K_{X}\right|$, and the base locus of the pencil $\left|-2 K_{W}\right|$ consists of the curves $C$ and $L$ such that $\alpha_{4} \circ \beta_{5} \circ \pi(C)$ is the unique base curve of the pencil $\left|-2 K_{X}\right|$, and the curve $\beta_{5} \circ \pi(L)$ is contained in the exceptional divisor of the morphism $\alpha_{4}$.

The the intersection form of the curves $C$ and $L$ on the surface $D$ is negatively definite, but the equivalence $\left.\mathcal{B}\right|_{D} \sim_{\mathbb{Q}} k C+k L$ holds, which is impossible by Lemmas 2.10 and 2.8 .

\section{Case $n=40$, hypersurface of Degree 19 in $\mathbb{P}(1,3,4,5,7)$.}

We use the notations and assumptions of Section 3. Let $n=40$. Then $X$ is a sufficiently general hypersurface in $\mathbb{P}(1,3,4,5,7)$ of degree 19 , the singularities of the hypersurface $X$ consist of the point $P_{1}$ that is a quotient singularity of type $\frac{1}{3}(1,1,2)$, the point $P_{2}$ that is a quotient singularity of type $\frac{1}{4}(1,1,3)$, the point $P_{3}$ that is a quotient singularity of type $\frac{1}{5}(1,2,3)$, and the point $P_{4}$ that is a quotient singularity of type $\frac{1}{7}(1,3,4)$, and $-K_{X}^{3}=19 / 420$.

There is a commutative diagram

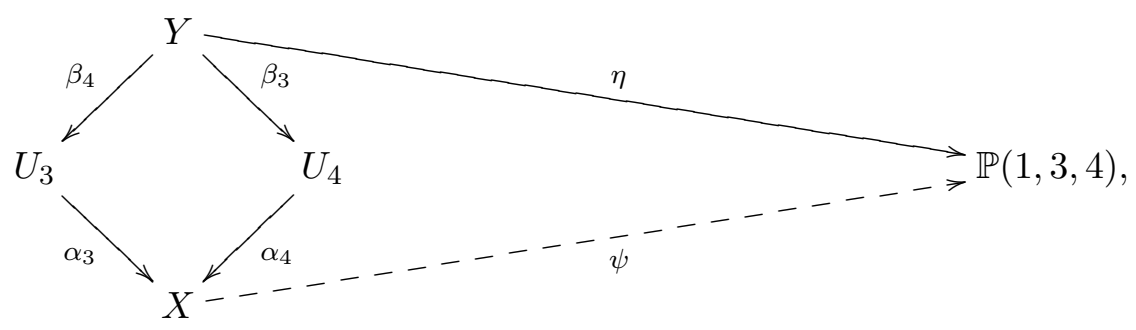

where $\psi$ is the natural projection, $\alpha_{3}$ is the weighted blow up of the singular point $P_{3}$ with weights $(1,2,3), \alpha_{4}$ is the weighted blow up of $P_{4}$ with weights $(1,3,4), \beta_{3}$ is the weighted blow up with weights $(1,2,3)$ of the proper transform of $P_{3}$ on $U_{4}, \beta_{4}$ is the weighted blow up with weights $(1,3,4)$ of the proper transform of $P_{4}$ on $U_{3}$, and $\eta$ is an elliptic fibration.

In the rest of the section we prove the following result.

Proposition 28.1. The claim of Theorem 1.10 holds for $n=40$.

It follows from Theorem 3.5. Lemma 3.14 and Proposition 3.7 that

$$
\varnothing \neq \mathbb{C} S\left(X, \frac{1}{k} \mathcal{M}\right) \subseteq\left\{P_{3}, P_{4}\right\} .
$$

Let $\mathcal{D}_{3}$ and $\mathcal{D}_{4}$ be the proper transforms of the linear system $\mathcal{M}$ on $U_{3}$ and $U_{4}$ respectively.

Lemma 28.2. Suppose that $\mathbb{C} S\left(X, \frac{1}{k} \mathcal{M}\right)=\left\{P_{3}, P_{4}\right\}$. Then there is a commutative diagram

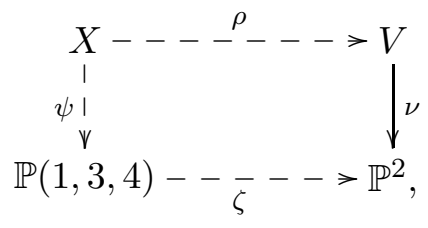

where $\zeta$ is a birational map.

Proof. Let $\mathcal{H}$ be the proper transform of the linear system $\mathcal{M}$ on the variety $Y$. Then it follows from Theorem 2.2 that the equivalence $\mathcal{H} \sim_{\mathbb{Q}}-k K_{Y}$ holds, which implies that $\mathcal{H}$ lies in the fibers of the elliptic fibration $\eta$, which implies the existence of the commutative diagram 28.3.

Let $P_{5}$ and $P_{6}$ be the singular points of the variety $U_{3}$ that are contained in the exceptional divisor of $\alpha_{3}$ such that $P_{5}$ and $P_{6}$ are quotient singularities of types $\frac{1}{2}(1,1,1)$ and $\frac{1}{3}(1,1,2)$ respectively, and $P_{7}$ and $P_{8}$ are the singular points of the variety $U_{4}$ that are quotient singularities of types $\frac{1}{3}(1,1,2)$ and $\frac{1}{4}(1,1,3)$ contained in the exceptional divisor of the morphism $\alpha_{4}$ 
respectively. Then it follows from Theorem 2.2 and Lemmas 2.1 and Lemma 2.3 that

$$
\left\{\begin{array}{l}
P_{4} \notin \mathbb{C S}\left(X, \frac{1}{k} \mathcal{M}\right) \Rightarrow \mathbb{C S}\left(U_{3}, \frac{1}{k} \mathcal{D}_{3}\right) \cap\left\{P_{5}, P_{6}\right\} \neq \varnothing, \\
P_{3} \notin \mathbb{C S}\left(X, \frac{1}{k} \mathcal{M}\right) \Rightarrow \mathbb{C S}\left(U_{4}, \frac{1}{k} \mathcal{D}_{4}\right) \cap\left\{P_{7}, P_{8}\right\} \neq \varnothing .
\end{array}\right.
$$

Lemma 28.4. Suppose that $P_{4} \notin \mathbb{C} \mathbb{S}\left(X, \frac{1}{k} \mathcal{M}\right)$. Then $P_{5} \notin \mathbb{C} \mathbb{S}\left(U_{3}, \frac{1}{k} \mathcal{D}_{3}\right)$.

Proof. Suppose that the set $\mathbb{C S}\left(U_{3}, \frac{1}{k} \mathcal{D}_{3}\right)$ contains the point $P_{5}$. Let $\gamma: W \rightarrow U_{3}$ be the weighted blow up of $P_{5}$ with weights $(1,1,1), F$ be the exceptional divisor of $\gamma, \mathcal{D}$ be the proper transform of the linear system $\mathcal{M}$ on the variety $W, \mathcal{H}$ be the proper transform of the pencil $\left|-3 K_{X}\right|$ on the variety $W, \bar{D}$ be a sufficiently general surface of the linear system $\mathcal{D}$, and $\bar{H}$ be a sufficiently general surface of the pencil $\mathcal{H}$. Then we have the equivalence

$$
\bar{H} \sim_{\mathbb{Q}}\left(\alpha_{3} \circ \gamma\right)^{*}\left(-3 K_{X}\right)-\frac{3}{5} \gamma^{*}(E)-\frac{1}{2} F,
$$

where $E$ is the exceptional divisor of the morphism $\alpha_{3}$. The base locus of the pencil $\mathcal{H}$ consists of the curve $\bar{C}$ such that $\alpha_{3} \circ \gamma(C)$ is the unique base curve of the pencil $\left|-3 K_{X}\right|$. On the other hand, it follows from Theorem 2.2 that $\bar{D} \sim_{\mathbb{Q}}-k K_{W}$.

The equivalence $\left.\bar{D}\right|_{\bar{H}} \sim_{\mathbb{Q}} k \bar{C}$ and the inequality $\bar{C}^{2}<0$ hold on the surface $\bar{H}$. It follows from Lemma 2.10 that the support of $\bar{H} \cdot \bar{D}$ consists of $\bar{C}$, which contradicts Lemma 2.8.

Lemma 28.5. Suppose that $P_{4} \notin \mathbb{C} \mathbb{S}\left(X, \frac{1}{k} \mathcal{M}\right)$. Then $P_{6} \notin \mathbb{C} \mathbb{S}\left(U_{3}, \frac{1}{k} \mathcal{D}_{3}\right)$.

Proof. Suppose that $P_{6} \in \mathbb{C S}\left(U_{3}, \frac{1}{k} \mathcal{D}_{3}\right)$. Let $\gamma: W \rightarrow U_{3}$ be the weighted blow up of $P_{6}$ with weights $(1,1,2), F$ and $G$ be the exceptional divisors of $\alpha_{3}$ and $\gamma$ respectively, $\mathcal{B}$ and $\mathcal{D}$ be the proper transforms of $\mathcal{M}$ and $\left|-7 K_{X}\right|$ on the variety $W$ respectively, and $D$ be a general surface of the linear system $\mathcal{D}$. Then it follows from Theorem 2.2 that $\mathcal{B} \sim_{\mathbb{Q}}-k K_{W}$, but the base locus of the linear system $\mathcal{D}$ does not contain curves. Moreover, we have

$$
D \sim_{\mathbb{Q}}\left(\alpha_{3} \circ \gamma\right)^{*}\left(-7 K_{X}\right)-\frac{2}{5} \gamma^{*}(F)-\frac{2}{3} G,
$$

the divisor $D$ is nef, but the explicit calculations imply that

$$
D \cdot B_{1} \cdot B_{2}=\left(\left(\alpha_{3} \circ \gamma\right)^{*}\left(-7 K_{X}\right)-\frac{2}{5} F-G\right)\left(\left(\alpha_{3} \circ \gamma\right)^{*}\left(-k K_{X}\right)-\frac{k}{5} F-\frac{k}{2} G\right)^{2}=-\frac{1}{12} k^{2},
$$

where $B_{1}$ and $B_{2}$ are general surfaces in $\mathcal{B}$, which is a contradiction.

Lemma 28.6. Suppose that $P_{3} \notin \mathbb{C S}\left(X, \frac{1}{k} \mathcal{M}\right)$. Then $P_{7} \notin \mathbb{C} \mathbb{S}\left(U_{4}, \frac{1}{k} \mathcal{D}_{4}\right)$.

Proof. Suppose that the set $\mathbb{C S}\left(U_{4}, \frac{1}{k} \mathcal{D}_{4}\right)$ contains the point $P_{7}$. Let $\gamma: W \rightarrow U_{4}$ be the weighted blow up of $P_{7}$ with weights $(1,1,2), F$ be the exceptional divisor of $\gamma, \mathcal{D}$ be the proper transform of the linear system $\mathcal{M}$ on $W, \mathcal{H}$ be the proper transform of $\left|-4 K_{X}\right|$ on $W, \bar{D}$ be a general surface of the linear system $\mathcal{D}$, and $\bar{H}$ be a general surface of the linear system $\mathcal{H}$. Then

$$
\bar{H} \sim_{\mathbb{Q}}\left(\alpha_{3} \circ \gamma\right)^{*}\left(-4 K_{X}\right)-\frac{4}{7} \gamma^{*}(E)-\frac{1}{3} F,
$$

and the base locus of $\mathcal{H}$ consists of the curve $\bar{C}$ such that $\alpha_{3} \circ \gamma(C)$ is the base curve of the linear system $\left|-4 K_{X}\right|$. It follows from Theorem 2.2 that the equivalence $\bar{D} \sim_{\mathbb{Q}}-k K_{W}$ holds.

The equality $\bar{C}^{2}=-1 / 30$ holds on the normal surface $\bar{H}$, which implies that the support of the cycle $\bar{H} \cdot \bar{D}$ consists of $\bar{C}$, because $\left.\bar{D}\right|_{\bar{H}} \sim_{\mathbb{Q}} k \bar{C}$, which contradicts Lemma 2.8.

Lemma 28.7. Suppose that $P_{3} \notin \mathbb{C} \mathbb{S}\left(X, \frac{1}{k} \mathcal{M}\right)$. Then $P_{8} \notin \mathbb{C} \mathbb{S}\left(U_{4}, \frac{1}{k} \mathcal{D}_{4}\right)$.

Proof. Suppose that the set $\mathbb{C} \mathbb{S}\left(U_{4}, \frac{1}{k} \mathcal{D}_{4}\right)$ contains the point $P_{8}$. Let $\gamma: W \rightarrow U_{4}$ be the weighted blow up of $P_{8}$ with weights $(1,1,3), \mathcal{D}$ be the proper transform of $\mathcal{M}$ on $W, \bar{H}$ be a general surface of the pencil $\left|-3 K_{W}\right|$, and $\bar{D}$ be a general surface in $\mathcal{D}$. Then $\bar{D} \sim_{\mathbb{Q}}-k K_{W}$, but the base locus of the pencil $\left|-3 K_{W}\right|$ consists of the irreducible curve $\bar{C}$ such that $\alpha_{4} \circ \gamma(C)$ is the base curve of $\left|-3 K_{X}\right|$. Moreover, the equality $\bar{C}^{2}=-1 / 20$ holds on $\bar{H}$, but $\left.\bar{D}\right|_{\bar{H}} \sim_{\mathbb{Q}} k \bar{C}$, which implies that the support of $\bar{H} \cdot \bar{D}$ consists of $\bar{C}$, which is impossible by Lemma 2.8 .

The claim Proposition 28.1 is proved. 
29. Case $n=43$, hypersurface of Degree 20 IN $\mathbb{P}(1,2,4,5,9)$.

We use the notations and assumptions of Section [3. Let $n=43$. Then $X$ is a sufficiently general hypersurface in $\mathbb{P}(1,2,4,5,9)$ of degree 20 , the equality $-K_{X}^{3}=1 / 18$ holds, and the singularities of the hypersurface $X$ consist of the points $P_{1}, P_{2}, P_{3}, P_{4}$ and $P_{5}$ that are quotient singularities of type $\frac{1}{2}(1,1,1)$, and the point $P_{6}$ that is a quotient singularity of type $\frac{1}{9}(1,4,5)$.

Proposition 29.1. The claim of Theorem 1.10 holds for $n=43$.

In the rest of the section we prove Proposition 29.1. It follows from Theorem 3.5 Lemma 3.14 and Proposition 3.7 that $\mathbb{C S}\left(X, \frac{1}{k} \mathcal{M}\right)=\left\{P_{6}\right\}$. There is a commutative diagram

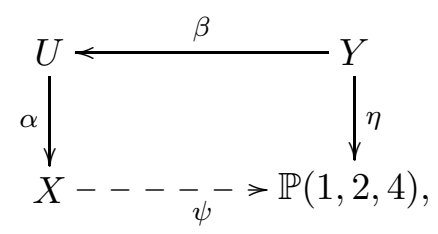

where $\psi$ is a projection, $\alpha$ is the weighted blow up of $P_{6}$ with weights $(1,4,5), \beta$ is the weighted blow up with weights $(1,1,4)$ of the singular point of the variety $U$ that is a quotient singularity of type $\frac{1}{5}(1,1,4)$ contained in the exceptional divisor of $\alpha$, and $\eta$ is an elliptic fibration.

Let $\mathcal{D}$ be the proper transform of the linear system $\mathcal{M}$ on the variety $U$, and $P_{7}$ and $P_{8}$ be the singular points of $U$ that are quotient singularities of types $\frac{1}{4}(1,1,3)$ and $\frac{1}{5}(1,1,4)$ contained in the exceptional divisor of $\alpha$ respectively. Then $\mathcal{D} \sim_{\mathbb{Q}}-k K_{U}$ by Theorem 2.2 .

We must show that the proper transform of the linear system $\mathcal{M}$ on the variety $Y$ is contained in the fibers of the $\eta$, which is implied by Theorem 2.2. if $P_{8} \in \mathbb{C} \mathbb{S}\left(U, \frac{1}{k} \mathcal{D}\right)$. Thus, to conclude the proof of Proposition 29.1 we may assume that $P_{8} \notin \mathbb{C S}\left(U, \frac{1}{k} \mathcal{D}\right)$.

Remark 29.2. The set $\mathbb{C S}\left(U, \frac{1}{k} \mathcal{D}\right)$ contains $P_{7}$ by Lemma 2.3 , because $-K_{U}$ is nef and big.

Let $\gamma: W \rightarrow U$ be the weighted blow up of the singular point $P_{7}$ with weights $(1,1,3), \mathcal{B}$ be the proper transform of the linear system $\mathcal{M}$ on the variety $W$, and $P_{9}$ be the singular point of the variety $W$ that is a quotient singularity of type $\frac{1}{3}(1,1,2)$ contained in the exceptional divisor of the morphism $\gamma$. Then the equivalence $\mathcal{B} \sim_{\mathbb{Q}}-k K_{W}$ holds by Theorem 2.2

Lemma 29.3. The set $\mathbb{C} S\left(W, \frac{1}{k} \mathcal{B}\right)$ does not contains the point $P_{9}$.

Proof. Suppose that the set $\mathbb{C S}\left(W, \frac{1}{k} \mathcal{B}\right)$ contains the point $P_{9}$. Let $\pi: Z \rightarrow W$ be the weighted blow up of the singular point $P_{9}$ with weights $(1,1,2), \mathcal{H}$ be the proper transform of the linear system $\mathcal{M}$ on the variety $Z$, and $\mathcal{P}$ be the proper transform of the linear system $\left|-5 K_{X}\right|$ on the variety $Z$. Then $\mathcal{H} \sim_{\mathbb{Q}}-k K_{Z}$ by Theorem [2.2] but the base locus of $\mathcal{P}$ consists of the irreducible curve $\Gamma$ such that $\alpha \circ \gamma \circ \pi(\Gamma)$ is the base curve in of $\left|-5 K_{X}\right|$.

Let $H_{1}$ and $H_{2}$ be general surfaces of the linear system $\mathcal{H}$, and $D$ be general surface of the linear system $\mathcal{P}$. Then $D \cdot \Gamma=1$ and $D^{3}=6$. Therefore, the divisor $D$ is nef and big, but the elementary computations imply that $D \cdot H_{1} \cdot H_{2}=0$, which is impossible by Corollary 2.7

Therefore, the claim of Lemma 2.3 implies the following corollary.

Corollary 29.4. The singularities of the $\log$ pair $\left(W, \frac{1}{k} \mathcal{B}\right)$ are terminal.

The hypersurface $X$ can be given by the quasihomogeneous equation of degree 20

$$
w^{2} y+w f_{11}(x, y, z, t)+f_{20}(x, y, z, t)=0 \subset \mathbb{P}(1,2,4,5,9) \cong \operatorname{Proj}(\mathbb{C}[x, y, z, t, w]),
$$

where $\operatorname{wt}(x)=1, \operatorname{wt}(y)=2, \operatorname{wt}(z)=4, \operatorname{wt}(t)=5, \operatorname{wt}(w)=9$, and $f_{i}(x, y, z, t)$ is a quasihomogeneous polynomial of degree $i$. Let $D$ be a general surface in $\left|-2 K_{X}\right|$, and $S$ be a surface that is cut on the hypersurface $X$ by the equation $x=0$. Then $D$ is cut on $X$ by the quasihomogeneous equation $\lambda x^{2}+\mu y=0$, where $(\lambda, \mu) \in \mathbb{P}^{1}$, and the base locus of $\left|-2 K_{X}\right|$ consists of the irreducible curve $C$ that is cut on the hypersurface $X$ by the equations $x=y=0$.

In the neighborhood of the point $P_{6}$, the monomials $x, z$ and $t$ can be considered as weighted local coordinates on $X$ such that $\operatorname{wt}(x)=1, \operatorname{wt}(z)=4$ and $\operatorname{wt}(t)=5$. Then in the neighborhood 
of the singular point $P_{7}$, the weighted blow up $\alpha$ is given by the equations

$$
x=\tilde{x} \tilde{z}^{\frac{1}{9}}, z=\tilde{z}^{\frac{4}{9}}, t=\tilde{t} z^{\frac{5}{9}},
$$

where $\tilde{x}, \tilde{z}$ and $\tilde{t}$ are weighted local coordinated on the variety $U$ in the neighborhood of the singular point $P_{7}$ such that $\operatorname{wt}(\tilde{x})=1, \operatorname{wt}(\tilde{z})=3$ and $\operatorname{wt}(\tilde{t})=1$.

Let $E$ be the exceptional divisor of the morphism $\alpha$, and $\tilde{D}, \tilde{S}$ and $\tilde{C}$ be the proper transforms on the variety $U$ of the surface $D$, the surface $S$ and the curve $C$ respectively. Then $E$ is given by the equation $\tilde{z}=0$, and the surface $\tilde{S}$ is given by the equation $\tilde{x}=0$. Moreover, it follows from the local equation of the surface $\tilde{D}$ that $\tilde{D} \cdot \tilde{S}=\tilde{C}+2 \tilde{L}_{1}$, where $\tilde{L}_{1}$ is the curve that is locally given by the equations $\tilde{z}=\tilde{x}=0$. Moreover, the surface $\tilde{D}$ is not normal in a general point of the curve $\tilde{L}_{1}$. Nevertheless, we have the equivalences

$$
\tilde{D} \sim_{\mathbb{Q}} 2 \tilde{S} \sim_{\mathbb{Q}} \alpha^{*}\left(-2 K_{X}\right)-\frac{2}{9} E .
$$

In the neighborhood of the point $P_{9}$ the morphism $\gamma$ is given by the equations

$$
\tilde{x}=\bar{x} \bar{z}^{\frac{1}{4}}, \tilde{z}=\bar{z}^{\frac{3}{4}}, \tilde{t}=\bar{t} \bar{z}^{\frac{1}{4}}
$$

where $\bar{x}, \bar{z}$ and $\bar{t}$ are weighted local coordinates on the variety $W$ in the neighborhood of the point $P_{9}$ such that $\operatorname{wt}(\bar{x})=1, \operatorname{wt}(\bar{z})=2$ and $\operatorname{wt}(\bar{t})=1$. In particular, the exceptional divisor of the morphism $\gamma$ is given by the equation $\bar{z}=0$, and the proper transform of the surface $S$ on the variety $W$ is given by the equation $\bar{x}=0$.

Let $F$ be the exceptional divisor of the morphism $\gamma$, and $\bar{D}, \bar{S}, \bar{E}, \bar{C}$ and $\bar{L}_{1}$ be the proper transforms on the variety $W$ of the surface $D$, the surface $S$, the surface $E$, the curve $C$ and the curve $\tilde{L}_{1}$ respectively. Then we the equivalence

$$
\left\{\begin{array}{l}
\bar{S} \sim_{\mathbb{Q}}-K_{W} \sim_{\mathbb{Q}}(\alpha \circ \gamma)^{*}\left(-K_{X}\right)-\frac{1}{9} \gamma^{*}(E)-\frac{1}{4} F, \\
\bar{D} \sim_{\mathbb{Q}}-2 K_{W} \sim_{\mathbb{Q}}(\alpha \circ \gamma)^{*}\left(-2 K_{X}\right)-\frac{2}{9} \gamma^{*}(E)-\frac{1}{2} F, \\
\bar{E} \sim_{\mathbb{Q}} \gamma^{*}(E)-\frac{3}{4} F .
\end{array}\right.
$$

Let $\bar{L}_{2}$ be the curve on the variety $W$ that is given by the equation $\bar{z}=\bar{x}=0$. Then

$$
\bar{D} \cdot \bar{S}=\bar{C}+2 \bar{L}_{1}+\bar{L}_{2}, \bar{D} \cdot \bar{E}=2 \bar{L}_{1}, \bar{D} \cdot F=2 \bar{L}_{2},
$$

but the base locus of $\left|-2 K_{W}\right|$ consists of the curves $\bar{C}, \bar{L}_{1}$ and $\bar{L}_{2}$. The equivalences 29.5imply

$$
\bar{D} \cdot \bar{C}=0, \bar{D} \cdot \bar{L}_{1}=-\frac{2}{5}, \bar{D} \cdot \bar{L}_{2}=\frac{2}{3} .
$$

Let $\bar{H}$ and $\bar{T}$ be the proper transforms on the variety $W$ of the surfaces that are cut on the hypersurface $X$ by the equations $y=0$ and $t=0$ respectively. Then

$$
\left\{\begin{array}{l}
\bar{T} \sim_{\mathbb{Q}}(\alpha \circ \gamma)^{*}\left(-5 K_{X}\right)-\frac{5}{9} \gamma^{*}(E)-\frac{1}{4} F \sim_{\mathbb{Q}}(\alpha \circ \gamma)^{*}\left(-5 K_{X}\right)-\frac{5}{9} \bar{E}-\frac{2}{3} F, \\
\bar{H} \sim_{\mathbb{Q}}(\alpha \circ \gamma)^{*}\left(-2 K_{X}\right)-\frac{11}{9} \gamma^{*}(E)-\frac{3}{2} F \sim_{\mathbb{Q}}(\alpha \circ \gamma)^{*}\left(-2 K_{X}\right)-\frac{11}{9} \bar{E}-\frac{5}{3} F,
\end{array}\right.
$$

which implies that

$$
-14 K_{W} \sim_{\mathbb{Q}} 14 \bar{D} \sim_{\mathbb{Q}} 2 \bar{T}+2 \bar{H}+2 \bar{E},
$$

and the support of the cycle $\bar{T} \cdot \bar{H}$ does not contain the curves $\bar{L}_{2}$ and $\bar{C}$. Therefore, the base locus of the linear system $\left|-14 K_{W}\right|$ does not contain curves except the curve $\bar{L}_{1}$.

The singularities of the mobile $\log$ pair $\left(W, \lambda\left|-14 K_{W}\right|\right)$ are log-terminal for some rational number $\lambda>1 / 14$, but the divisor $K_{W}+\lambda\left|-14 K_{W}\right|$ has non-negative intersection with all curves on the variety $W$ except the curve $\bar{L}_{1}$. It follows from [13] that the log-flip $\zeta: W \rightarrow Z$ in the curve $\bar{L}_{1}$ with respect to the log pair $\left(W, \lambda\left|-14 K_{W}\right|\right)$ exists.

Let $\mathcal{P}$ be the proper transform of the linear system $\mathcal{M}$ on the variety $Z$. Then the singularities of the log pair $\left(Z, \frac{1}{k} \mathcal{P}\right)$ are terminal, because the singularities of the log pair $\left(W, \frac{1}{k} \mathcal{B}\right)$ are terminal, but the rational map $\zeta$ is a $\log$ flop with respect to the $\log$ pair $\left(W, \frac{1}{k} \mathcal{B}\right)$, but $-K_{Z}$ is numerically 
effective, because the base locus of the linear system $\left|-14 K_{W}\right|$ does not contain curves outside the curve $\bar{L}_{1}$, and the inequality $-K_{W} \cdot \bar{L}_{1}<0$ holds.

In the rest of the section we show that $-K_{Z}$ is big, which contradicts Lemma 2.1

The rational functions $y / x^{2}$ and $t y / x^{7}$ are contained in $|2 S|$ and $|7 S|$ respectively, but the equivalences 29.6 implies that $y / x^{2}$ and $t y / x^{7}$ are contained in $|2 \bar{S}|$ and $|7 \bar{S}|$ respectively.

Let $\bar{Z}$ be the proper transform on the variety $W$ of the irreducible surface that is cut on the hypersurface $X$ by the equation $z=0$. Then the equivalences

$$
\bar{Z} \sim_{\mathbb{Q}}(\alpha \circ \gamma)^{*}\left(-4 K_{X}\right)-\frac{4}{9} \gamma^{*}(E) \sim_{\mathbb{Q}}(\alpha \circ \gamma)^{*}\left(-4 K_{X}\right)-\frac{4}{9} \bar{E}-\frac{1}{3} F
$$

hold, which imply that $-6 K_{W} \sim_{\mathbb{Q}} \bar{Z}+\bar{H}+\bar{E}$. Thus, the rational function $z y / x^{6}$ is contained in the linear system $|6 \bar{S}|$. Thus, the linear system $\left|-42 K_{W}\right|$ maps the variety $W$ dominantly on some three-dimensional variety, which implies that the divisor $-K_{Z}$ is big.

\section{Case $n=44$, hypersurface of Degree 20 in $\mathbb{P}(1,2,5,6,7)$.}

We use the notations and assumptions of Section 3. Let $n=44$. Then $X$ is a sufficiently general hypersurface in $\mathbb{P}(1,2,5,6,7)$ of degree 20 , the equality $-K_{X}^{3}=1 / 21$ holds, and the singularities of the hypersurface $X$ consist of the points $P_{1}, P_{2}$ and $P_{3}$ that are quotient singularities of type $\frac{1}{2}(1,1,1)$, the point $P_{4}$ that is a quotient singularity of type $\frac{1}{6}(1,1,5)$, and the point $P_{5}$ that is a quotient singularity of type $\frac{1}{7}(1,2,5)$. There is a commutative diagram

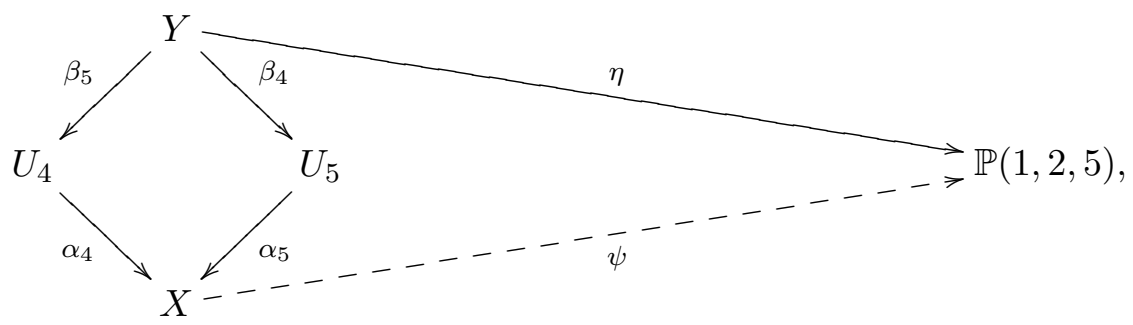

where $\psi$ is a projection, $\alpha_{4}$ is the weighted blow up of $P_{4}$ with weights $(1,1,5), \alpha_{5}$ is the weighted blow up of $P_{5}$ with weights $(1,2,5), \beta_{4}$ is the weighted blow up with weights $(1,1,5)$ of the proper transform of $P_{4}$ on $U_{5}, \beta_{5}$ is the weighted blow up with weights $(1,2,5)$ of the proper transform of the point $P_{5}$ on the variety $U_{4}$, and $\eta$ is an elliptic fibration. There is a commutative diagram

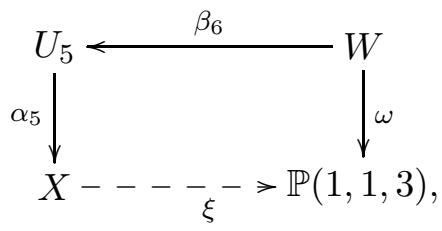

where $\xi$ is a projection, $\beta_{6}$ is the weighted blow up with weights $(1,2,3)$ of the singular point of $U_{5}$ that is a singularity of type $\frac{1}{5}(1,2,3)$ contained in the $\alpha_{5}$-exceptional divisor, and $\omega$ is an elliptic fibration.

Proposition 30.1. Either there is a commutative diagram

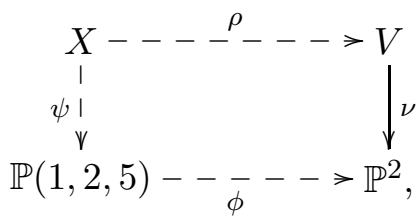

or there is a commutative diagram

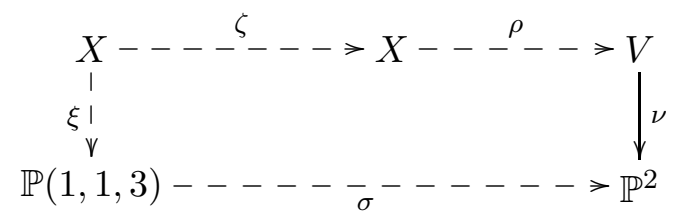

where $\phi, \zeta$ and $\sigma$ are birational maps. 
Let us prove Proposition 30.1. It follows from Lemma 3.14 and Proposition 3.7 that

$$
\varnothing \neq \mathbb{C} \mathbb{S}\left(X, \frac{1}{k} \mathcal{M}\right) \subseteq\left\{P_{4}, P_{5}\right\},
$$

but the proof of Lemma 18.2 implies that the set $\mathbb{C S}\left(X, \frac{1}{k} \mathcal{M}\right)$ contains the point $P_{5}$.

The existence of the commutative diagram 30.2 easily follows from Theorem 2.2 in the case when $\mathbb{C S}\left(X, \frac{1}{k} \mathcal{M}\right)=\left\{P_{4}, P_{5}\right\}$. Thus, to conclude the proof of Proposition 30.1 we may assume that the set $\mathbb{C} S\left(X, \frac{1}{k} \mathcal{M}\right)$ consists of the point $P_{5}$.

In the rest of the section we prove the existence of the commutative diagram 30.3 ,

Let $\mathcal{D}_{5}$ be the proper transform of $\mathcal{M}$ on $U_{5}$. Then $\mathcal{D}_{5} \sim_{\mathbb{Q}}-k K_{U_{5}}$ by Theorem 2.2, which implies that the set $\mathbb{C} \mathbb{S}\left(U_{5}, \frac{1}{k} \mathcal{D}_{5}\right)$ is not empty by Lemma 2.1] Let $G$ be the exceptional divisor of the morphism $\alpha_{5}$, and $\bar{P}_{6}$ and $\bar{P}_{7}$ are the singular points of $G$ that are quotient singularities of types $\frac{1}{5}(1,2,3)$ and $\frac{1}{2}(1,1,1)$ on the variety $U_{5}$ respectively.

In the case when the set $\mathbb{C S}\left(U_{5}, \frac{1}{k} \mathcal{D}_{5}\right)$ contains the point $\bar{P}_{6}$, the existence of the commutative diagram 30.3 follows from Theorem [2.2. Therefore, to conclude the proof of Proposition 30.1 . we may assume that the set $\mathbb{C S}\left(U_{5}, \frac{1}{k} \mathcal{D}_{5}\right)$ contains the point $\bar{P}_{7}$ by Lemma 2.3 .

Remark 30.4. The linear system $\left|-5 K_{U_{5}}\right|$ is a proper transform of $\left|-5 K_{X}\right|$, and the base locus of the linear system $\left|-5 K_{U_{5}}\right|$ consists of the irreducible curve that is the fiber of the rational map $\psi \circ \alpha_{5}$ passing through the point $\bar{P}_{7}$.

Let $\pi: U \rightarrow U_{5}$ be the weighted blow up of $\bar{P}_{7}$ with weights $(1,1,1), F$ be the exceptional divisor of $\pi, \mathcal{D}$ be the proper transform of $\mathcal{M}$ on the variety $U$, and $\mathcal{H}$ be the proper transform of the linear system $\left|-5 K_{U_{5}}\right|$ on the variety $U$. Then $\mathcal{D} \sim_{\mathbb{Q}}-k K_{U}$ by Theorem 2.2, but

$$
\mathcal{H} \sim_{\mathbb{Q}} \pi^{*}\left(-5 K_{U_{5}}\right)-\frac{1}{2} F,
$$

and the base locus of $\mathcal{H}$ consists of the irreducible curve $Z$ such that $\alpha_{5} \circ \pi(Z)$ is the unique curve in the base locus of the linear system $\left|-5 K_{X}\right|$.

Let $S$ be a general surface of the linear system $\mathcal{H}$. Then the equality $S \cdot Z=1 / 3$ holds, which implies that the divisor $\pi^{*}\left(-10 K_{U_{5}}\right)-F$ is nef. Let $D_{1}$ and $D_{2}$ be general surfaces in $\mathcal{D}$. Then

$$
-\frac{2 k^{2}}{3}=\left(\pi^{*}\left(-10 K_{U_{5}}\right)-F\right) \cdot\left(\pi^{*}\left(-k K_{U_{5}}\right)-\frac{k}{2} F\right)^{2}=\left(\pi^{*}\left(-10 K_{U_{5}}\right)-F\right) \cdot D_{1} \cdot D_{2} \geqslant 0,
$$

which is a contradiction. The claim of Proposition 30.1 is proved.

$$
\text { 31. Case } n=47 \text {, hypersurface of Degree } 21 \text { in } \mathbb{P}(1,1,5,7,8) \text {. }
$$

We use the notations and assumptions of Section 3 Let $n=47$. Then $X$ is a general hypersurface in $\mathbb{P}(1,1,5,7,8)$ of degree 21 , whose singularities consist of the point $P_{1}$ that is a singularity of type $\frac{1}{5}(1,2,3)$, and the point $P_{2}$ that is a singularity of type $\frac{1}{8}(1,1,7)$.

There is a commutative diagram

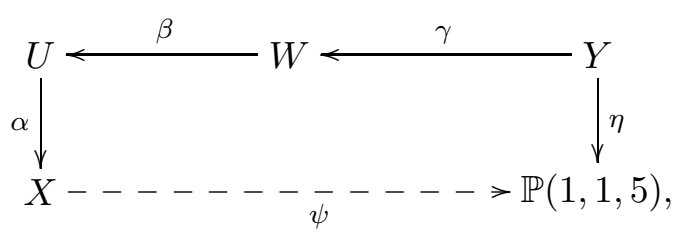

where $\alpha$ is the weighted blow up of the point $P_{2}$ with weights $(1,1,7), \beta$ is the weighted blow up with weights $(1,1,6)$ of the singular point of the variety $U$ that is a quotient singularity of type $\frac{1}{7}(1,1,6), \gamma$ is the weighted blow up with weights $(1,1,5)$ of the singular point $W$ that is a quotient singularity of type $\frac{1}{6}(1,1,5)$, and $\eta$ is and elliptic fibration.

Proposition 31.1. The claim of Theorem 1.10 holds for $n=47$.

In the rest of the section we prove Proposition 31.1] It follows from Theorem 3.5 Lemma 3.14 and Proposition 3.7 that $\mathbb{C S}\left(X, \frac{1}{k} \mathcal{M}\right) \subseteq\left\{P_{1}, P_{2}\right\}$. 
The hypersurface $X$ can be given by the equation

$$
w^{2} z+\sum_{i=0}^{2} w z^{i} g_{13-5 i}(x, y, t)+\sum_{i=0}^{3} z^{i} g_{21-5 i}(x, y, t)=0 \subset \operatorname{Proj}(\mathbb{C}[x, y, z, t, w]),
$$

where $\operatorname{wt}(x)=1, \operatorname{wt}(y)=1, \operatorname{wt}(z)=5, \operatorname{wt}(t)=7, \operatorname{wt}(w)=8$, and $g_{i}(x, y, t)$ is a quasihomogeneous polynomial of degree $i$.

Lemma 31.2. The set $\mathbb{C S}\left(X, \frac{1}{k} \mathcal{M}\right)$ contains the point $P_{2}$.

Proof. Suppose that $\mathbb{C S}\left(X, \frac{1}{k} \mathcal{M}\right)$ does not contains $P_{2}$. Then $\mathbb{C S}\left(X, \frac{1}{k} \mathcal{M}\right)=\left\{P_{1}\right\}$.

Let $\pi: Z \rightarrow X$ be the weighted blow up of $P_{1}$ with weights $(1,2,3), E$ be the exceptional divisor of $\pi$, and $\mathcal{B}$ be the proper transform of $\mathcal{M}$ on $Z$. Then $E \cong \mathbb{P}(1,2,35)$ and $\mathcal{B} \sim_{\mathbb{Q}}-k K_{Z}$.

Let $\bar{P}_{3}$ and $\bar{P}_{4}$ be the singular points of the variety $Z$ contained in $E$ that are singularities of types $\frac{1}{2}(1,1,1)$ and $\frac{1}{3}(1,1,2)$ respectively. Then the proof of Proposition 22.1 implies that the singularities of the log pair $\left(Z, \frac{1}{k} \mathcal{B}\right)$ are terminal. However, the divisor $-K_{Z}$ is not nef.

The base locus of the pencil $\left|-K_{Z}\right|$ consists of the irreducible curves $C$ and $L$ such that the curve $\pi(C)$ is cut out by $x=y=0$, the curve $L$ is contained in the divisor $E$, the curve $L$ is contained in $\left|\mathcal{O}_{\mathbb{P}(1,2,3)}(1)\right|$, the inequalities $-K_{Z} \cdot C<0$ and $-K_{Z} \cdot L>0$ hold.

It follows from [13] that the antiflip $\zeta: Z \rightarrow \bar{Z}$ in the curve $C$ exists, and $-K_{\bar{Z}}$ is nef.

Let $\mathcal{P}$ be the proper transform of the linear system $\mathcal{M}$ on the variety $\bar{Z}$. Then the singularities of the log pair $\left(\bar{Z}, \frac{1}{k} \mathcal{P}\right)$ are terminal, because the singularities of the log pair $\left(Z, \frac{1}{k} \mathcal{B}\right)$ are terminal, and the antiflip $\zeta$ is a $\log$ flop with respect to the $\log$ pair $\left(Z, \frac{1}{k} \mathcal{B}\right)$.

One can easily check that the rational functions $y / x, z y / x^{6}, t y / x^{8}$ and $y w / x^{9}$ are contained in the linear system $\left|-a K_{Z}\right|$, where $a=1,6,8$ and 9 respectively. Therefore, the complete linear system $\left|-72 K_{Z}\right|$ induces the birational map $\chi: Z \rightarrow \bar{X}$ such that $\bar{X}$ is a hypersurface of degree 24 in $\mathbb{P}(1,1,6,8,9)$. Hence, the divisor $-K_{\bar{Z}}$ is big, which contradicts Lemma 2.1.

Let $G$ be the exceptional divisor of the morphism $\alpha, \mathcal{D}$ be the proper transform of the linear system $\mathcal{M}$ on the variety $U, \bar{P}_{1}$ be the proper transform of $P_{1}$ on $U$, and $\bar{P}_{5}$ be the singular point of the variety $U$ that is contained in $G$. Then $\mathcal{D} \sim_{\mathbb{Q}}-k K_{U}$ by Theorem 2.2 ,

Lemma 31.3. The set $\mathbb{C S}\left(U, \frac{1}{k} \mathcal{D}\right)$ contains the point $\bar{P}_{5}$.

Proof. Suppose that $\bar{P}_{5} \notin \mathbb{C S}\left(U, \frac{1}{k} \mathcal{D}\right)$. Then $\mathbb{C S}\left(U, \frac{1}{k} \mathcal{D}\right)=\left\{\bar{P}_{1}\right\}$ by Lemmas 2.1 and 2.3.

Let $\pi: Z \rightarrow U$ be the weighted blow up of the point $\bar{P}_{1}$ with weights $(1,2,3), E$ be the exceptional divisor of the morphism $\pi$, and $\bar{G}$ and $\mathcal{B}$ be the proper transforms of $G$ and $\mathcal{M}$ on the variety $Z$ respectively. Then $\mathcal{B} \sim_{\mathbb{Q}}-k K_{Z}$ by Theorem 2.2. but the proof of Lemma 31.2 implies that the singularities of the log pair $\left(Z, \frac{1}{k} \mathcal{B}\right)$ are terminal.

Let $S_{x}, S_{y}, S_{z}, S_{t}$ and $S_{w}$ be the proper transforms on the variety $Z$ of the surfaces that are cut on $X$ by the equations $x=0, y=0, z=0, t=0$ and $w=0$ respectively. Then

$$
\left\{\begin{array}{l}
S_{x} \sim_{\mathbb{Q}}(\alpha \circ \pi)^{*}\left(-K_{X}\right)-\frac{1}{5} E-\frac{1}{8} \bar{G}, \\
S_{y} \sim_{\mathbb{Q}}(\alpha \circ \pi)^{*}\left(-K_{X}\right)-\frac{6}{5} E-\frac{1}{8} \bar{G}, \\
S_{z} \sim_{\mathbb{Q}}(\alpha \circ \pi)^{*}\left(-5 K_{X}\right)-\frac{1}{5} E-\frac{13}{8} \bar{G}, \\
S_{t} \sim_{\mathbb{Q}}(\alpha \circ \pi)^{*}\left(-7 K_{X}\right)-\frac{2}{5} E-\frac{7}{8} \bar{G}, \\
S_{w} \sim_{\mathbb{Q}}(\alpha \circ \pi)^{*}\left(-8 K_{X}\right)-\frac{3}{5} E .
\end{array}\right.
$$

The base locus of the pencil $\left|-K_{Z}\right|$ consists of the irreducible curves $C$ and $L$ such that the curve $\alpha \circ \pi(C)$ is cut by the equation $x=y=0$ on the hypersurface $X$, the curve $L$ is contained in the divisor $E$, and the curve $L$ is the unique curve of the linear system $\left|\mathcal{O}_{\mathbb{P}(1,2,3)}(1)\right|$.

It follows from 13 , that there is the antiflip $\zeta: Z \rightarrow \bar{Z}$ in $C$ such that $-K_{\bar{Z}}$ is nef.

Let $\mathcal{P}$ be the proper transform of the linear system $\mathcal{M}$ on the variety $\bar{Z}$. Then the singularities of the $\log$ pair $\left(\bar{Z}, \frac{1}{k} \mathcal{P}\right)$ are terminal. 
The equivalences 31.4 imply that the functions $y / x, z y / x^{6}, t y / x^{8}$ and $w z y^{2} / x^{15}$ are contained in the complete linear system $\left|a S_{x}\right|$, where $a=1,6,8$ and 15 respectively. Hence, the linear system $\left|-120 K_{Z}\right|$ induces the birational map $\chi: Z \rightarrow \bar{X}$ such that $\bar{X}$ is a hypersurface of degree 30 in $\mathbb{P}(1,1,6,8,15)$, which implies that $-K_{\bar{Z}}$ is big, which contradicts Lemma 2.1

Remark 31.5. It follows from Lemma 2.4 that $\mathbb{C S}\left(U, \frac{1}{k} \mathcal{D}\right)=\left\{\bar{P}_{5}\right\}$.

Let $\mathcal{H}$ be the proper transform of the linear system $\mathcal{M}$ on the variety $W, F$ be the exceptional divisor of $\beta, \tilde{P}_{1}$ be the proper transform of $P_{1}$ on $W$, and $\tilde{P}_{6}$ be the singular point of $W$ that is contained in $F$. Then $\mathcal{H} \sim_{\mathbb{Q}}-k K_{W}$ by Theorem 2.2 , but $\mathbb{C} \mathbb{S}\left(W, \frac{1}{k} \mathcal{H}\right) \neq \varnothing$.

Lemma 31.6. Suppose that $\tilde{P}_{6} \in \mathbb{C} \mathbb{S}\left(W, \frac{1}{k} \mathcal{H}\right)$. Then there is a commutative diagram

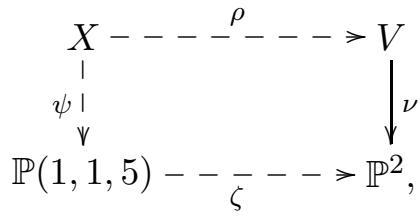

where $\zeta$ is a birational map.

Proof. The existence of the diagram 31.7 follows from Theorem 2.2

We may assume that the set $\mathbb{C} \mathbb{S}\left(W, \frac{1}{k} \mathcal{H}\right)$ consists of the point $\tilde{P}_{1}$ by Lemma 2.3 .

Let $\pi: Z \rightarrow W$ be the weighted blow up of $\tilde{P}_{1}$ with weights $(1,2,3), E$ be the exceptional divisor of $\pi, \mathcal{B}$ be the proper transform of $\mathcal{M}$ on $Z$, and $\tilde{G}$ and $\tilde{F}$ be the proper transforms on the variety $Z$ of the surfaces $G$ and $F$ respectively. Then it follows from the proof of Lemma 31.2 that the singularities of the $\log$ pair $\left(Z, \frac{1}{k} \mathcal{B}\right)$ are terminal, but $\mathcal{B} \sim_{\mathbb{Q}}-k K_{Z}$ by Theorem 2.2

Let $S_{x}, S_{y}, S_{z}, S_{t}$ and $S_{w}$ be proper transforms on the variety $Z$ of the surfaces that are cut on the variety $X$ by the equations $x=0, y=0, z=0, t=0$ and $w=0$ respectively. Then

$$
\left\{\begin{array}{l}
S_{x} \sim_{\mathbb{Q}}(\alpha \circ \beta \circ \pi)^{*}\left(-K_{X}\right)-\frac{1}{5} E-\frac{1}{8} \tilde{G}-\frac{1}{4} \tilde{F}, \\
S_{y} \sim_{\mathbb{Q}}(\alpha \circ \beta \circ \pi)^{*}\left(-K_{X}\right)-\frac{6}{5} E-\frac{1}{8} \tilde{G}-\frac{1}{4} \tilde{F}, \\
S_{z} \sim_{\mathbb{Q}}(\alpha \circ \beta \circ \pi)^{*}\left(-5 K_{X}\right)-\frac{1}{5} E-\frac{13}{8} \tilde{G}-\frac{9}{4} \tilde{F}, \\
S_{t} \sim_{\mathbb{Q}}(\alpha \circ \beta \circ \pi)^{*}\left(-7 K_{X}\right)-\frac{2}{5} E-\frac{7}{8} \tilde{G}-\frac{3}{4} \tilde{F}, \\
S_{w} \sim_{\mathbb{Q}}(\alpha \circ \beta \circ \pi)^{*}\left(-8 K_{X}\right)-\frac{3}{5} E .
\end{array}\right.
$$

The equivalences 31.8 imply that the functions $y / x, z y / x^{6}, t z y^{2} / x^{14}$ and $w z^{2} y^{3} / x^{21}$ are contained in the linear systems $\left|S_{x}\right|,\left|6 S_{x}\right|,\left|14 S_{x}\right|$ and $\left|21 S_{x}\right|$ respectively. Therefore, the complete linear system $\left|-42 K_{Z}\right|$ induces the birational map $\chi: Z \rightarrow \bar{X}$ such that $\bar{X}$ is a threefold ${ }^{5}$.

The base locus of the linear system $\left|-42 K_{Z}\right|$ consists of the irreducible curve $C$ such that the curve $\alpha \circ \beta \circ \pi(C)$ is cut on $X$ by the equations $x=y=0$. Therefore, the existence of the antiflip $\zeta: Z \rightarrow \bar{Z}$ in the curve $C$ follows from [13], which implies that $-K_{\bar{Z}}$ is nef and big.

The rational map $\zeta$ is a log flip with respect to the $\log$ pair $\left(Z, \frac{1}{k} \mathcal{B}\right)$. Therefore, we see that the singularities of the mobile log pair $\left(\bar{Z}, \frac{1}{k} \mathcal{P}\right)$ are terminal, where $\mathcal{P}$ is the proper transform of the linear system $\mathcal{M}$ on the variety $Z$, which is impossible by Lemma 2.1 .

The claim of Proposition 31.1 is proved.

\footnotetext{
${ }^{5}$ The proofs of Lemmas 31.2 and 31.3 give the birational transformations of the hypersurface $X$ into hypersurfaces in $\mathbb{P}(1,1,6,8,9)$ and $\mathbb{P}(1,1,6,8,15)$ of degrees 24 and 30 respectively. The anticanonical models of the varieties $U$ and $W$ are hypersurfaces in $\mathbb{P}(1,1,5,7,13)$ and $\mathbb{P}(1,1,5,12,18)$ of degrees 26 and 36 respectively, and the threefold $\bar{X}$ is a hypersurface in $\mathbb{P}(1,1,6,14,21)$ of degree 42 . Up to to the action of $\operatorname{Bir}(X)$, there are no other non-trivial birational transformations of $X$ into Fano threefolds with canonical singularities.
} 
32. Case $n=48$, hypersurface of Degree 21 In $\mathbb{P}(1,2,3,7,9)$.

We use the notations and assumptions of Section 3 Let $n=48$. Then $X$ is a general hypersurface in $\mathbb{P}(1,2,3,7,9)$ of degree 21 , whose singularities consist of the point $P_{1}$ that is a singularity of type $\frac{1}{2}(1,1,1)$, the points $P_{2}$ and $P_{3}$ that are singularity of type $\frac{1}{3}(1,1,2)$, and the point $P_{4}$ that is a singularity of type $\frac{1}{9}(1,2,7)$. There is a commutative diagram

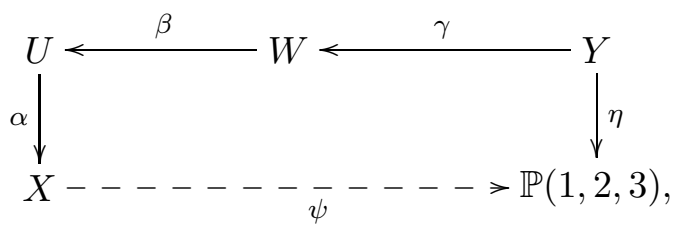

where $\psi$ is a projection, $\alpha$ is the weighted blow up of $P_{4}$ with weights $(1,2,7), \beta$ is the weighted blow up with weights $(1,2,5)$ of the singular point of the variety $U$ that is a quotient singularity of type $\frac{1}{7}(1,2,5)$ contained in the exceptional divisor of $\alpha, \gamma$ is the weighted blow up with weights $(1,2,3)$ of the singular point of $W$ that is a singularity of type $\frac{1}{5}(1,2,3)$ contained in the exceptional divisor of $\beta$, and $\eta$ is an elliptic fibration.

Proposition 32.1. The claim of Theorem 1.10 holds for $n=48$.

In the rest of the section we prove Proposition 32.1. It follows from Theorem 3.5 Lemma 3.14 and Proposition 3.7 that $\mathbb{C S}\left(X, \frac{1}{k} \mathcal{M}\right)=\left\{P_{4}\right\}$.

Let $E$ be the $\alpha$-exceptional divisor, $\mathcal{D}$ be the proper transform of $\mathcal{M}$ on $U$, and $P_{5}$ and $P_{6}$ be the singular points of $U$ contained in $E$ that are singularities of types $\frac{1}{2}(1,1,1)$ and $\frac{1}{7}(1,2,5)$ respectively. Then $E \cong \mathbb{P}(1,2,7)$, but $\mathcal{D} \sim_{\mathbb{Q}}-k K_{U}$ by Theorem 2.2

It follows from Lemmas 2.12 .3 and 2.4 that $\mathbb{C S}\left(U, \frac{1}{k} \mathcal{D}\right) \subseteq\left\{P_{5}, P_{6}\right\}$.

Lemma 32.2. The set $\mathbb{C} S\left(U, \frac{1}{k} \mathcal{D}\right)$ does not contain the point $P_{5}$.

Proof. Suppose that the set $\mathbb{C S}\left(U, \frac{1}{k} \mathcal{D}\right)$ contains the point $P_{5}$. Let $\pi: Z \rightarrow U$ be the weighted blow up of $P_{5}$ with weights $(1,1,1), G$ be the exceptional divisor of $\pi$, and $\mathcal{B}$ and $\mathcal{P}$ be the proper transforms of the linear systems $\mathcal{M}$ and $\left|-7 K_{X}\right|$ on the variety $Z$ respectively. Then

$$
\mathcal{B} \sim_{\mathbb{Q}}-k K_{Z} \sim_{\mathbb{Q}}(\alpha \circ \pi)^{*}\left(-k K_{X}\right)-\frac{k}{9} \pi^{*}(E)-\frac{k}{2} G
$$

by Theorem 2.2 but the base locus of the linear system $\mathcal{P}$ does not contain curves. Let $H$ be a general divisor of the linear system $\mathcal{P}$. Then the divisor $H$ is numerically effective, but

$H \cdot B_{1} \cdot B_{2}=\left((\alpha \circ \pi)^{*}\left(-k K_{X}\right)-\frac{k}{9} \pi^{*}(E)-\frac{k}{2} G\right)^{2}\left((\alpha \circ \pi)^{*}\left(-7 K_{X}\right)-\frac{7}{9} \pi^{*}(E)-\frac{1}{2} G\right)=-\frac{1}{6} k^{2}$, where $B_{1}$ and $B_{2}$ are general surfaces of the linear system $\mathcal{B}$, which is a contradiction.

Hence, the set $\mathbb{C} S\left(U, \frac{1}{k} \mathcal{D}\right)$ consists of the point $P_{6}$.

Let $F$ be the $\beta$-exceptional divisor, $\mathcal{H}$ be the proper transform of $\mathcal{M}$ on $W$, and $P_{7}$ and $P_{8}$ be the singular points of $W$ contained in $F$ that are singularities of types $\frac{1}{2}(1,1,1)$ and $\frac{1}{5}(1,2,3)$ respectively. Then $F \cong \mathbb{P}(1,2,5)$, but $\mathcal{H} \sim_{\mathbb{Q}}-k K_{W}$ by Theorem 2.2.

It follows from Lemmas 2.1$] 2.3$ and 2.4 that $\mathbb{C S}\left(W, \frac{1}{k} \mathcal{H}\right) \subseteq\left\{P_{7}, P_{8}\right\}$.

Lemma 32.3. Suppose that $P_{8} \in \mathbb{C} S\left(W, \frac{1}{k} \mathcal{H}\right)$. Then there is a commutative diagram

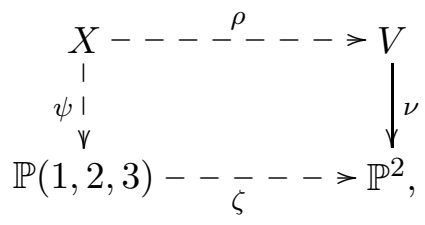

where $\zeta$ is a birational map.

Proof. Let $S$ be the proper transform on the variety $Y$ of a sufficiently general surface of the linear system $\mathcal{M}$, and $\Gamma$ be a general fiber of $\eta$. Then $S \sim_{\mathbb{Q}}-k K_{Y}$ by Theorem 2.2, which implies that $S \cdot \Gamma=0$. Therefore, the surface $S$ lies in the fibers of the fibration $\eta$, which implies the existence of the commutative diagram 32.4 . 
To conclude the proof of Proposition 32.1, we may assume that $P_{7} \in \mathbb{C} \mathbb{S}\left(W, \frac{1}{k} \mathcal{H}\right)$.

Let $\pi: Z \rightarrow W$ be the weighted blow up of $P_{7}$ with weights $(1,1,1), G$ be the exceptional divisor of $\pi$, and $\mathcal{B}$ be the proper transform of $\mathcal{M}$ on $Z$. Then $\mathcal{B} \sim_{\mathbb{Q}}-k K_{Z}$ by Theorem 2.2.

The hypersurface $X$ can be given by the equation

$$
w^{2} z+w f_{12}(x, y, z, t)+f_{21}(x, y, z, t)=0 \subset \mathbb{P}(1,2,3,7,9) \cong \operatorname{Proj}(\mathbb{C}[x, y, z, t, w]),
$$

where $\operatorname{wt}(x)=1, \operatorname{wt}(y)=2, \operatorname{wt}(z)=3, \operatorname{wt}(t)=7, \operatorname{wt}(w)=9$, and $f_{i}(x, y, z, t)$ is a quasihomogeneous polynomial of degree $i$.

Let $\mathcal{P}$ be the proper transform on the variety $Z$ of the pencil of surfaces that are cut on the hypersurface $X$ by the equations $\lambda x^{3}+\mu z=0$, where $(\lambda, \mu) \in \mathbb{P}^{1}$. Then the base locus of the pencil $\mathcal{P}$ consists of the irreducible curves $C, L_{1}$ and $L_{2}$ such that $\alpha \circ \beta \circ \pi(C)$ is the curve that is cut on the hypersurface $X$ by the equations $x=z=0$, the curve $\beta \circ \pi\left(L_{1}\right)$ is contained in the exceptional divisor $E$, the curve $\beta \circ \pi\left(L_{1}\right)$ is the unique curve in the base locus of the linear system $\left|\mathcal{O}_{\mathbb{P}(1,2,7)}(1)\right|$, the curve $\pi\left(L_{2}\right)$ is contained in $F$, and the curve $\pi\left(L_{2}\right)$ is the unique curve of the linear system $\left|\mathcal{O}_{\mathbb{P}(1,2,5)}(1)\right|$.

Let $D$ be a general surface of the pencil $\mathcal{P}, \bar{E}$ and $\bar{F}$ be the proper transforms of the exceptional divisors $E$ and $F$ on the variety $Z$ respectively, and $S$ be the proper transform on $Z$ of the surface that is cut on the hypersurface $X$ by the equation $x=0$. Then

$$
S \cdot D=C+L_{1}+L_{2}, \bar{E} \cdot D=3 L_{1}, \bar{F} \cdot D=3 L_{2},
$$

the surface $D$ is normal, and

$$
\left\{\begin{array}{l}
\bar{F} \sim_{\mathbb{Q}} \pi^{*}(F)-\frac{1}{2} G, \\
\bar{E} \sim_{\mathbb{Q}}(\beta \circ \pi)^{*}(E)-\frac{5}{7} \pi^{*}(F)-\frac{1}{2} G, \\
D \sim_{\mathbb{Q}}(\alpha \circ \beta \circ \pi)^{*}\left(-3 K_{X}\right)-\frac{3}{9}(\beta \circ \pi)^{*}(E)-\frac{3}{7} \pi^{*}(F)-\frac{3}{2} G, \\
S \sim_{\mathbb{Q}}(\alpha \circ \beta \circ \pi)^{*}\left(-K_{X}\right)-\frac{1}{9}(\beta \circ \pi)^{*}(E)-\frac{1}{7} \pi^{*}(F)-\frac{1}{2} G .
\end{array}\right.
$$

Consider the curves $C, L_{1}$ and $L_{2}$ as divisors on $\bar{D}$. The equivalences 32.5 imply that

$$
C \cdot C=L_{1} \cdot L_{1}=-\frac{1}{2}, L_{2} \cdot L_{2}=-\frac{2}{5}, C \cdot L_{1}=C \cdot L_{2}=L_{1} \cdot L_{2}=0,
$$

which implies that the intersection form of the curves $C, L_{1}$ and $L_{2}$ on the normal surface $D$ is negatively defined. On the other hand, we have

$$
\left.B\right|_{D} \sim_{\mathbb{Q}}-\left.\left.k K_{Z}\right|_{D} \sim_{\mathbb{Q}} k S\right|_{D} \sim_{\mathbb{Q}} k C+k L_{1}+k L_{2},
$$

where $B$ is a general surface of the linear system $\mathcal{B}$, which contradicts Lemmas 2.10 and 2.8

The claim of Proposition 32.1 is proved.

33. Case $n=49$, hypersurface of Degree 21 in $\mathbb{P}(1,3,5,6,7)$.

We use the notations and assumptions of Section 3 Let $n=49$. Then $X$ is a general hypersurface in $\mathbb{P}(1,3,5,6,7)$ of degree 21 , whose singularities consist of the points $P_{1}, P_{2}$ and $P_{3}$ that are singularities of type $\frac{1}{3}(1,1,2)$, the point $P_{4}$ that is a singularity of type $\frac{1}{5}(1,2,3)$, and the point $P_{5}$ that is a singularity of type $\frac{1}{6}(1,1,5)$.

There is a commutative diagram

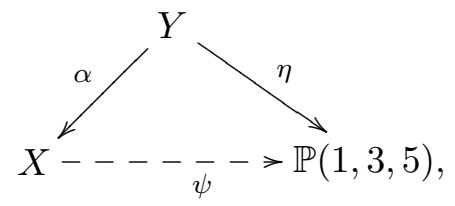

where $\psi$ is a projection, $\alpha$ is the blow up of $P_{5}$ with weights $(1,1,5)$, and $\eta$ is an elliptic fibration. 
There is a commutative diagram

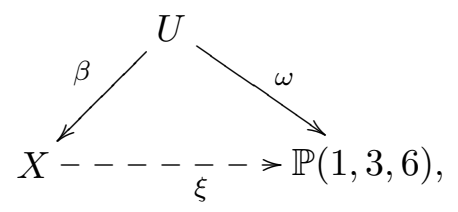

where $\xi$ is a projection, $\beta$ is the blow up of $P_{4}$ with weights $(1,2,3)$, and $\omega$ is an elliptic fibration.

Proposition 33.1. Either there is a commutative diagram

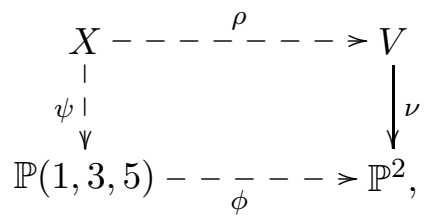

or there is a commutative diagram

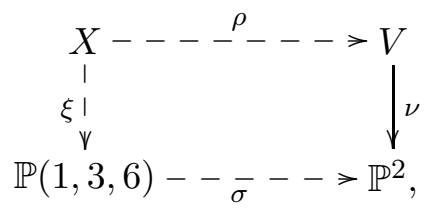

where $\phi$ and $\sigma$ are birational maps.

Proof. It follows from Proposition 3.7 and Lemma 3.14 that $\mathbb{C} S\left(X, \frac{1}{k} \mathcal{M}\right) \subseteq\left\{P_{4}, P_{5}\right\}$.

Suppose that the set $\mathbb{C S}\left(X, \frac{1}{k} \mathcal{M}\right)$ contains point $P_{4}$. Let $\mathcal{D}$ be the proper transform of the linear system $\mathcal{M}$ on the variety $U$. Then $\mathcal{D} \sim_{\mathbb{Q}}-k K_{U}$ by Theorem 2.2 . Intersecting a general surface of the linear system $\mathcal{D}$ with a general fiber of $\omega$, we see that $\mathcal{D}$ lies in the fibers of the elliptic $\omega$, which implies the existence of the commutative diagram 33.3

Similarly, the commutative diagram 33.2 exists when $P_{4} \in \mathbb{C} S\left(X, \frac{1}{k} \mathcal{M}\right)$.

34. Case $n=51$, hypersurface of Degree 22 in $\mathbb{P}(1,1,4,6,11)$.

We use the notations and assumptions of Section 3 Let $n=51$. Then $X$ is a hypersurface of degree 22 in $\mathbb{P}(1,1,4,6,11)$, the equality $-K_{X}^{3}=1 / 12$ holds, and the singularities of $X$ consist of the point $P_{1}$ that is a quotient singularity of type $\frac{1}{2}(1,1,1)$, the point $P_{2}$ that is a quotient singularity of type $\frac{1}{3}(1,1,4)$, and the point $P_{3}$ that is a quotient singularity of type $\frac{1}{6}(1,1,5)$.

The hypersurface $X$ is birationally superrigid. There is a commutative diagram

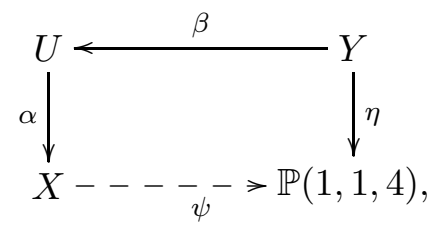

where $\psi$ is a projection, $\alpha$ is the weighted blow up of $P_{3}$ with weights $(1,1,5), \beta$ is the weighted blow up with weights $(1,1,4)$ of the singular point of the variety $U$ that is contained in the exceptional divisor of $\alpha$, and $\eta$ is an elliptic fibration. There is a commutative diagram

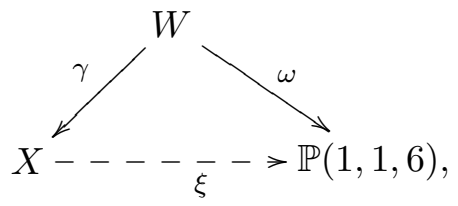

where $\xi$ is a projection, $\gamma$ is the blow up of $P_{2}$ with weights $(1,1,3)$, and $\omega$ is an elliptic fibration. 
Proposition 34.1. Either there is a commutative diagram

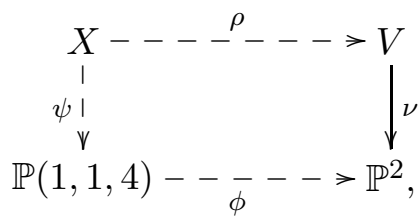

or there is a commutative diagram

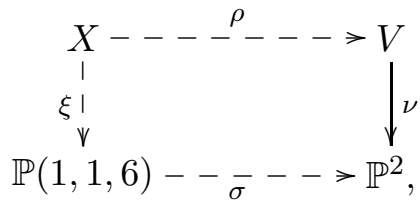

where $\phi$ and $\sigma$ are birational maps.

Proof. Suppose that $P_{2}$ is contained in $\mathbb{C S}\left(X, \frac{1}{k} \mathcal{M}\right)$. Let $\mathcal{D}$ be the proper transform of $\mathcal{M}$ on the variety $W$. Then the equivalence $\mathcal{D} \sim_{\mathbb{Q}}-k K_{W}$ holds by Theorem 2.2, which implies the existence of the commutative diagram 34.3 .

We may assume that $\mathbb{C S}\left(X, \frac{1}{k} \mathcal{M}\right)=\left\{P_{3}\right\}$ by Theorem 3.5. Proposition 3.7 and Lemma 3.14.

Let $\mathcal{B}$ be the proper transform of $\mathcal{M}$ on $U$. Then $\mathcal{B} \sim_{\mathbb{Q}}-k K_{U}$ by Theorem 2.2 but the anticanonical divisor $-K_{U}$ is nef and big. It follows from Lemmas 2.1 and 2.3 that the set of centers of canonical singularities $\mathbb{C} \mathbb{S}\left(U, \frac{1}{k} \mathcal{B}\right)$ contains the singular point of the variety $U$ that is contained in the exceptional divisor of the birational morphism $\alpha$.

Let $\mathcal{H}$ be the proper transform of $\mathcal{M}$ on $Y$. Then $\mathcal{H} \sim \mathbb{Q}-k K_{Y}$ by Theorem 2.2,

Intersecting a general surface of the linear system $\mathcal{H}$ with a general fiber of the fibration $\eta$, we see that the linear system $\mathcal{H}$ lies in the fibers of the elliptic fibration $\eta$, which implies the existence of the commutative diagram 34.2 .

35. Case $n=56$, hypersurface of Degree 24 in $\mathbb{P}(1,2,3,8,11)$.

We use the notations and assumptions of Section 3 Let $n=56$. Then $X$ is a general hypersurface in $\mathbb{P}(1,2,3,8,11)$ of degree 24 , whose singularities consist of the points $P_{1}, P_{2}$ and $P_{3}$ that are singularities of type $\frac{1}{2}(1,1,1)$, and the point $P_{4}$ that is a singularity of type $\frac{1}{11}(1,3,8)$.

There is a commutative diagram

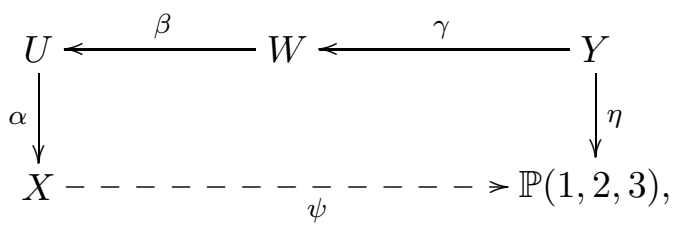

where $\psi$ is a projection, $\alpha$ is the weighted blow up of $P_{4}$ with weights $(1,3,8), \beta$ is the weighted blow up with weights $(1,3,5)$ of the point of $U$ that is a quotient singularity of type $\frac{1}{8}(1,3,5)$ contained in the exceptional divisor of $\alpha, \gamma$ is the weighted blow up with weights $(1,2,3)$ of the point of $W$ that is a quotient singularity of type $\frac{1}{5}(1,2,3)$ contained in the exceptional divisor of the morphism $\beta$, and $\eta$ is an elliptic fibration.

Proposition 35.1. The claim of Theorem 1.10 holds for $n=56$.

Let us prove Proposition 35.1. It follows from Proposition 3.7 that $\mathbb{C S}\left(X, \frac{1}{k} \mathcal{M}\right)=\left\{P_{4}\right\}$.

Let $E$ be the exceptional divisor of the morphism $\alpha, \mathcal{D}$ be the proper transform of the linear system $\mathcal{M}$ on the variety $U$, and $P_{5}$ and $P_{6}$ be the singular point of $U$ contained in $E$ that are singularities of types $\frac{1}{3}(1,1,2)$ and $\frac{1}{8}(1,3,5)$ respectively. Then $\mathcal{D} \sim_{\mathbb{Q}}-k K_{U}$ by Theorem 2.2

Lemma 35.2. The set $\mathbb{C} S\left(U, \frac{1}{k} \mathcal{D}\right)$ does not contain the point $P_{5}$.

Proof. Suppose that the set $\mathbb{C S}\left(U, \frac{1}{k} \mathcal{D}\right)$ contains the point $P_{5}$. Let $\pi: Z \rightarrow U$ be the weighted blow up of $P_{5}$ with weights $(1,1,2), G$ be the exceptional divisor of $\pi$, and $\mathcal{B}$ and $\mathcal{P}$ be the 
proper transforms of $\mathcal{M}$ and $\left|-8 K_{X}\right|$ on the variety $Z$ respectively. Then $\mathcal{B} \sim_{\mathbb{Q}}-k K_{Z}$, but the base locus of the linear system $\mathcal{P}$ does not contain curves.

Let $H$ be a general surface in $\mathcal{P}$. Then the divisor $H$ is nef and big. In particular, we have

$H \cdot B_{1} \cdot B_{2}=\left((\alpha \circ \pi)^{*}\left(-k K_{X}\right)-\frac{k}{11} \pi^{*}(E)-\frac{k}{3} G\right)^{2}\left((\alpha \circ \pi)^{*}\left(-8 K_{X}\right)-\frac{8}{11} \pi^{*}(E)-\frac{2}{3} G\right)=0$,

where $B_{1}$ and $B_{2}$ are general surfaces in $\mathcal{B}$, which contradicts Corollary 2.7

Hence, the set $\mathbb{C S}\left(U, \frac{1}{k} \mathcal{D}\right)$ consists of the point $P_{6}$ by Lemma 2.3

Let $F$ be the exceptional divisor of the morphism $\beta, \mathcal{H}$ be the proper transform of the linear system $\mathcal{M}$ on the variety $W$, and $P_{7}$ and $P_{8}$ be the singular points of $W$ that are singularities of types $\frac{1}{3}(1,1,2)$ and $\frac{1}{5}(1,2,3)$ contained in $F$ respectively. Then $\mathcal{H} \sim_{\mathbb{Q}}-k K_{W}$ by Theorem 2.2 ,

The proof of Lemma 32.3 implies the existence of the commutative diagram

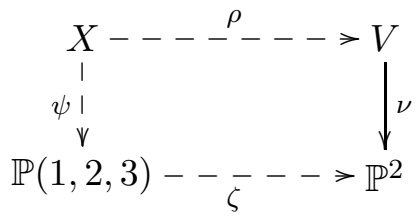

in the case when $P_{8} \in \mathbb{C} \mathbb{S}\left(W, \frac{1}{k} \mathcal{H}\right)$, where $\zeta$ is a birational map.

We may assume that $\mathbb{C} S\left(W, \frac{1}{k} \mathcal{H}\right)=\left\{P_{7}\right\}$ due to Lemmas 2.1 and 2.3.

Let $\pi: Z \rightarrow W$ be the weighted blow up of $P_{7}$ with weights $(1,1,2), G$ be the exceptional divisor of $\pi$, and $\mathcal{B}$ be the proper transform of $\mathcal{M}$ on $Z$. Then $\mathcal{B} \sim_{\mathbb{Q}}-k K_{Z}$ by Theorem 2.2.

Lemma 35.3. The singularities of the $\log \operatorname{pair}\left(Z, \frac{1}{k} \mathcal{B}\right)$ are terminal.

Proof. Suppose that $\mathbb{C S}\left(Z, \frac{1}{k} \mathcal{B}\right) \neq \varnothing$. Let $P_{9}$ be the singular point of $G$. Then $P_{9}$ is a singularity of type $\frac{1}{2}(1,1,1)$ on $Z$, the set $\mathbb{C S}\left(Z, \frac{1}{k} \mathcal{B}\right)$ contains $P_{9}$ by Lemma 2.3 and $G \cong \mathbb{P}(1,1,2)$.

Let $\bar{\pi}: \bar{Z} \rightarrow Z$ be the weighted blow up of the point $P_{9}$ with weights $(1,1,1)$, and $\bar{G}$ be the exceptional divisor of $\bar{\pi}$. Take any divisor $D$ on the variety $\bar{Z}$ such that the equivalence

$$
D \sim_{\mathbb{Q}}-2 K_{\bar{Z}}-(\beta \circ \pi \circ \bar{\pi})^{*}\left(16 K_{U}\right)-(\pi \circ \bar{\pi})^{*}\left(18 K_{W}\right)
$$

holds. Analyzing the base locus of the pencil $\left|-2 K_{\bar{Z}}\right|$, we see that $D$ is nef, but $D^{3}>0$.

The divisor $D$ is nef and big, but $D \cdot \bar{H}_{1} \cdot \bar{H}_{2}=0$, where $\bar{H}_{1}$ and $\bar{H}_{2}$ are the proper transforms on $\bar{Z}$ of general surfaces in $\mathcal{M}$, which is impossible by Corollary 2.7

The hypersurface $X$ can be given by the equation

$$
w^{2} y+w f_{13}(x, y, z, t)+f_{24}(x, y, z, t)=0 \subset \mathbb{P}(1,2,3,8,11) \cong \operatorname{Proj}(\mathbb{C}[x, y, z, t, w]),
$$

where $\operatorname{wt}(x)=1, \operatorname{wt}(y)=2, \operatorname{wt}(z)=3, \operatorname{wt}(t)=8, \operatorname{wt}(w)=11$, and $f_{i}(x, y, z, t)$ is a sufficiently general quasihomogeneous polynomial of degree $i$.

Let $\bar{E}$ and $\bar{F}$ be the proper transforms on the variety $Z$ of the divisors $E$ and $F$ respectively, and $S_{1}, S_{2}, S_{3}$ and $S_{8}$ be the proper transforms on the variety $Z$ of the surfaces that are cut on the hypersurface $X$ by the equations $x=0, y=0, z=0$ and $t=0$ respectively. Then

$$
\left\{\begin{array}{l}
\bar{F} \sim_{\mathbb{Q}} \pi^{*}(F)-\frac{1}{3} G, \\
\bar{E} \sim_{\mathbb{Q}}(\beta \circ \pi)^{*}(E)-\frac{5}{8} \pi^{*}(F)-\frac{2}{3} G, \\
S_{1} \sim_{\mathbb{Q}}(\alpha \circ \beta \circ \pi)^{*}\left(-K_{X}\right)-\frac{1}{11}(\beta \circ \pi)^{*}(E)-\frac{1}{8} \pi^{*}(F)-\frac{1}{3} G, \\
S_{2} \sim_{\mathbb{Q}}(\alpha \circ \beta \circ \pi)^{*}\left(-2 K_{X}\right)-\frac{13}{11}(\beta \circ \pi)^{*}(E)-\frac{5}{8} \pi^{*}(F)-\frac{2}{3} G, \\
S_{3} \sim_{\mathbb{Q}}(\alpha \circ \beta \circ \pi)^{*}\left(-3 K_{X}\right)-\frac{3}{11}(\beta \circ \pi)^{*}(E)-\frac{3}{8} \pi^{*}(F)-\frac{1}{3} G, \\
S_{8} \sim_{\mathbb{Q}}(\alpha \circ \beta \circ \pi)^{*}\left(-8 K_{X}\right)-\frac{8}{11}(\beta \circ \pi)^{*}(E) .
\end{array}\right.
$$


The base locus of $\left|-2 K_{Z}\right|$ consists of the irreducible curves $C, L_{1}, L_{2}$ and $L_{3}$ such that the curve $\alpha \circ \beta \circ \pi(C)$ is cut on $X$ by the equations $x=y=0$, the curve $\beta \circ \pi\left(L_{1}\right)$ is contained in the divisor $E$, the curve $\beta \circ \pi\left(L_{1}\right)$ is contained in $\left|\mathcal{O}_{\mathbb{P}(1,3,8)}(1)\right|$, the curve $\pi\left(L_{2}\right)$ is contained in the divisor $F$, the curve $\pi\left(L_{2}\right)$ is contained in $\left|\mathcal{O}_{\mathbb{P}(1,3,5)}(1)\right|$, the curve $L_{3}$ is contained in the divisor $G$, the curve $L_{3}$ is contained in $\left|\mathcal{O}_{\mathbb{P}(1,1,2)}(1)\right|$. Moreover, we have

$$
S_{1} \cdot D=C+2 L_{1}+2 L_{2}+L_{3}, \bar{E} \cdot D=2 L_{1}, \bar{F} \cdot D=2 L_{2}, G \cdot D=2 L_{3},
$$

where $D$ is a general surface in $\left|-2 K_{Z}\right|$. It follows from the equivalences 35.4 that

$$
-K_{Z} \cdot C=\frac{1}{10},-K_{Z} \cdot L_{1}=-\frac{1}{3},-K_{Z} \cdot L_{2}=-\frac{1}{10},-K_{Z} \cdot L_{3}=\frac{1}{2},
$$

which implies that the curves $L_{1}$ and $L_{2}$ are the only curves on the variety $Z$ that have negative intersection with the divisor $-K_{Z}$.

The singularities of the log pair $\left(Z, \lambda\left|-2 K_{Z}\right|\right)$ are log-terminal for some rational $\lambda>1 / 2$, but the divisor $K_{Z}+\lambda\left|-2 K_{Z}\right|$ has nonnegative intersection with all curves on the variety $Z$ except the curves $L_{1}$ and $L_{2}$. It follows from [13] that there is a composition of antiflips $\zeta: Z \rightarrow \bar{Z}$ such that the divisor $-K_{\bar{Z}}$ is numerically effective.

Let $\mathcal{P}$ be the proper transform of the linear system $\mathcal{M}$ on the variety $\bar{Z}$. Then the singularities of the log pair $\left(\bar{Z}, \frac{1}{k} \mathcal{P}\right)$ are terminal, because the singularities of the log pair $\left(Z, \frac{1}{k} \mathcal{B}\right)$ are terminal, and the rational map $\zeta$ is a $\log$ flop with respect to the $\log$ pair $\left(Z, \frac{1}{k} \mathcal{B}\right)$.

It follows from the equivalences 35.4 that $-K_{Z} \sim_{\mathbb{Q}} S_{1}$ and

$$
\left\{\begin{array}{l}
S_{1} \sim_{\mathbb{Q}}(\alpha \circ \beta \circ \pi)^{*}\left(-K_{X}\right)-\frac{1}{11} \bar{E}-\frac{2}{11} \bar{F}-\frac{5}{11} G, \\
S_{2} \sim_{\mathbb{Q}}(\alpha \circ \beta \circ \pi)^{*}\left(-2 K_{X}\right)-\frac{13}{11} \bar{E}-\frac{15}{11} \bar{F}-\frac{21}{11} G, \\
S_{3} \sim_{\mathbb{Q}}(\alpha \circ \beta \circ \pi)^{*}\left(-3 K_{X}\right)-\frac{3}{11} \bar{E}-\frac{6}{11} \bar{F}-\frac{4}{11} G, \\
S_{8} \sim_{\mathbb{Q}}(\alpha \circ \beta \circ \pi)^{*}\left(-8 K_{X}\right)-\frac{8}{11} \bar{E}-\frac{5}{11} \bar{F}-\frac{7}{11} G .
\end{array}\right.
$$

The equivalences 35.5 implies that the rational functions $y / x^{2}, z y / x^{5}$ and $t y^{3} / x^{14}$ are contained in the linear systems $\left|2 S_{1}\right|,\left|5 S_{1}\right|$ and $\left|14 S_{1}\right|$ respectively. In particular, the complete linear system $\left|-70 K_{Z}\right|$ induces the dominant rational map $Z \rightarrow \mathbb{P}(1,2,5,14)$, which implies that the anticanonical divisor $-K_{\bar{Z}}$ is nef and big, which contradicts Lemma 2.1

36. Case $n=58$, hypersurface of Degree 24 in $\mathbb{P}(1,3,4,7,10)$.

We use the notations and assumptions of Section 3 Let $n=58$. Then $X$ is a hypersurface of degree 24 in $\mathbb{P}(1,3,4,7,10)$, the equality $-K_{X}^{3}=1 / 35$ holds, and the singularities of $X$ consist of the points $P_{1}, P_{2}$ and $P_{3}$ of type $\frac{1}{2}(1,1,1), \frac{1}{7}(1,3,4)$ and $\frac{1}{10}(1,3,7)$ respectively.

There is a commutative diagram

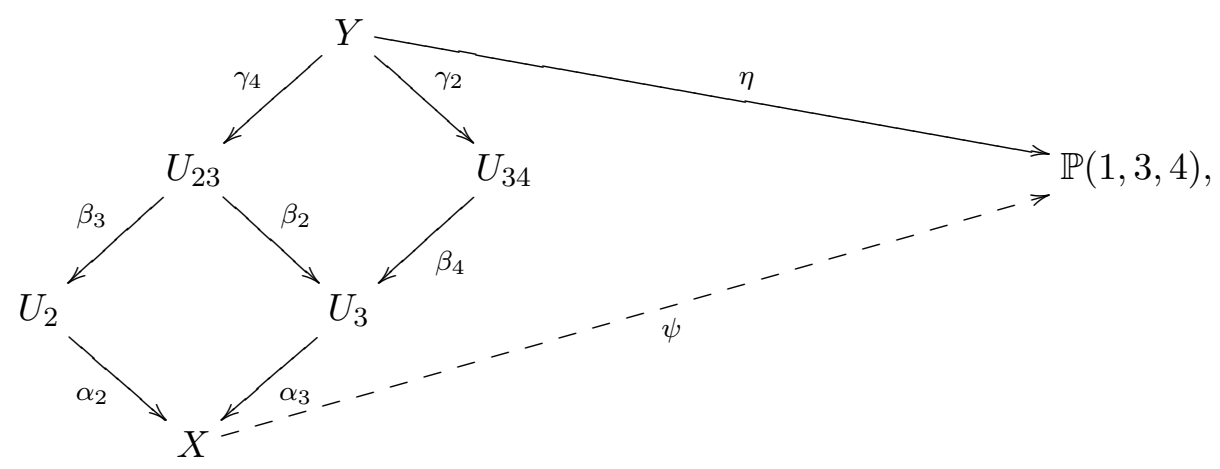

where $\psi$ is a projection, $\alpha_{2}$ is the weighted blow up of $P_{2}$ with weights $(1,3,4), \alpha_{3}$ is the weighted blow up of $P_{3}$ with weights $(1,3,7), \beta_{3}$ is the weighted blow up with weights $(1,3,7)$ of the proper transform of $P_{3}$ on $U_{2}, \beta_{2}$ is the weighted blow up with weights $(1,3,4)$ of the proper transform of the point $P_{2}$ on $U_{2}, \beta_{4}$ is the weighted blow up with weights $(1,3,4)$ of the singular point of the variety $U_{3}$ that is a quotient singularity of type $\frac{1}{7}(1,3,4)$ contained in the exceptional divisor 
of the morphism $\alpha_{3}, \gamma_{2}$ is the weighted blow up with weights $(1,3,4)$ of the proper transform of the point $P_{2}$ on $U_{34}, \gamma_{4}$ is the weighted blow up with weights $(1,3,4)$ of the singular point of the variety $U_{23}$ that is a quotient singularity of type $\frac{1}{7}(1,3,4)$ contained in the exceptional divisor of the morphism $\beta_{3}$, and $\eta$ is an elliptic fibration.

In the rest of the section we prove the following result.

Proposition 36.1. The claim of Theorem 1.10 holds for $n=58$.

It follows from Theorem 3.5. Lemma 3.14 and Proposition 3.7 that $\mathbb{C S}\left(X, \frac{1}{k} \mathcal{M}\right) \subseteq\left\{P_{2}, P_{3}\right\}$.

Lemma 36.2. The set $\mathbb{C S}\left(X, \frac{1}{k} \mathcal{M}\right)$ contains the point $P_{3}$.

Proof. Suppose that the set $\mathbb{C} \mathbb{S}\left(X, \frac{1}{k} \mathcal{M}\right)$ does not contain the point $P_{3}$. Let $\mathcal{D}_{2}$ be the proper transform of the linear system $\mathcal{M}$ on the variety $U_{2}$, and $O$ and $Q$ be the singular points of the variety $U_{2}$ contained in the exceptional divisor of $\alpha_{2}$ such that the points $O$ and $Q$ are singularities of types $\frac{1}{3}(1,1,2)$ and $\frac{1}{4}(1,1,3)$ respectively. Then $\mathcal{D}_{2} \sim_{\mathbb{Q}}-k K_{U_{2}}$ by Theorem 2.2 .

Suppose that $O \in \mathbb{C} S\left(U_{2}, \frac{1}{k} \mathcal{D}_{2}\right)$. Let $\pi: W \rightarrow U_{2}$ be the weighted blow up of the singular point $O$ with weights $(1,1,2), \mathcal{B}$ and $\mathcal{P}$ be the proper transforms of $\mathcal{M}$ and $\left|-4 K_{X}\right|$ on the variety $W$ respectively, and $S$ be a general surface in $\mathcal{P}$. Then the base locus of $\mathcal{P}$ consists of an irreducible curve $C$ such that $\alpha_{2}(C)$ is the base curve of $\left|-4 K_{X}\right|$, but $C^{2}<0$ on the normal surface $S$, which contradicts $\left.\mathcal{B}\right|_{S} \sim_{\mathbb{Q}} k C$ by Lemmas 2.8 and 2.10 .

We see that $Q \in \mathbb{C} S\left(U_{2}, \frac{1}{k} \mathcal{D}_{2}\right)$ by Lemma 2.3, because $\mathbb{C} S\left(U_{2}, \frac{1}{k} \mathcal{D}_{2}\right) \neq \varnothing$ by Lemma 2.1 .

Let $\zeta: U \rightarrow U_{2}$ be the weighted blow up of the point $Q$ with weights $(1,1,3), \mathcal{H}$ be the proper transform of $\mathcal{M}$ on the variety $U, H$ be a general surface of the linear system $\mathcal{H}$, and $D$ be a general surface in $\left|-3 K_{U}\right|$. Then $D$ is normal, and the base locus of the pencil $\left|-3 K_{U}\right|$ consists of the irreducible curve $Z$ such that $\alpha_{2}(Z)$ is the unique base curve of the pencil $\left|-3 K_{X}\right|$.

The equivalence $\left.\mathcal{H}\right|_{D} \sim_{\mathbb{Q}} k Z$ holds by Theorem [2.2, and the inequality $Z^{2}<0$ holds on the surface $D$, which is impossible by Lemmas 2.8 and 2.10.

Let $\mathcal{D}_{2}$ and $\mathcal{D}_{23}$ be the proper transforms of $\mathcal{M}$ on $U_{2}$ and $U_{23}$ respectively. The arguments of the proof of Lemma 36.2 implies the following two corollaries.

Corollary 36.3. Suppose that $\mathcal{D}_{2} \sim_{\mathbb{Q}}-k K_{U_{2}}$. Then the set $\mathbb{C} \mathbb{S}\left(U_{2}, \frac{1}{k} \mathcal{D}_{2}\right)$ does not contain subvarieties of the variety $U_{2}$ that are contained in the exceptional divisor of the morphism $\alpha_{2}$.

Corollary 36.4. Suppose that $\mathcal{D}_{23} \sim_{\mathbb{Q}}-k K_{U_{23}}$. Then the set $\mathbb{C} \mathbb{S}\left(U_{23}, \frac{1}{k} \mathcal{D}_{23}\right)$ does not contain subvarieties of the variety $U_{23}$ that are contained in the exceptional divisor of the morphism $\beta_{2}$.

Let $\mathcal{D}_{3}$ and $\mathcal{D}_{23}$ be the proper transforms of $\mathcal{M}$ on $U_{3}$ and $U_{34}$ respectively. Then we have the equivalence $\mathcal{D}_{3} \sim_{\mathbb{Q}}-k K_{U_{3}}$ by Theorem 2.2

Lemma 36.5. The set $\mathbb{C S}\left(X, \frac{1}{k} \mathcal{M}\right)$ contains the point $P_{2}$.

Proof. Suppose that $P_{2} \notin \mathbb{C S}\left(X, \frac{1}{k} \mathcal{M}\right)$. Then $\mathcal{D}_{3} \sim_{\mathbb{Q}}-k K_{U_{2}}$ by Theorem 2.2, which implies that the set $\mathbb{C} S\left(U_{3}, \frac{1}{k} \mathcal{D}_{3}\right)$ is not empty by Lemma 2.1

Let $P_{4}$ and $P_{5}$ be the singular points of the variety $U_{3}$ that are contained in the exceptional divisor of the birational morphism $\alpha_{3}$ such that the points $P_{4}$ and $P_{5}$ are quotient singularities of types $\frac{1}{7}(1,3,4)$ and $\frac{1}{3}(1,1,2)$ respectively. Then the set $\mathbb{C} \mathbb{S}\left(U_{3}, \frac{1}{k} \mathcal{D}_{3}\right)$ contains either the singular point $P_{4}$, or the singular point $P_{5}$ by Lemma 2.3 .

It follows from Lemma 36.2 that the set $\mathbb{C S}\left(U_{3}, \frac{1}{k} \mathcal{D}_{3}\right)$ does not contain the point $P_{5}$, which implies that $\mathbb{C} S\left(U_{3}, \frac{1}{k} \mathcal{D}_{3}\right)$ contains $P_{4}$. Hence, the equivalence $\mathcal{D}_{34} \sim_{\mathbb{Q}}-k K_{U_{34}}$ holds by Theorem 2.2. and the set $\mathbb{C S}\left(U_{34}, \frac{1}{k} \mathcal{D}_{34}\right)$ is not empty by Lemma 2.1.

Let $P_{6}$ and $P_{7}$ be the singular points of the variety $U_{34}$ that are contained in the exceptional divisor of the birational morphism $\beta_{4}$ such that the points $P_{6}$ and $P_{7}$ are quotient singularities of types $\frac{1}{3}(1,1,2)$ and $\frac{1}{4}(1,1,3)$ respectively. Then the set $\mathbb{C S}\left(U_{34}, \frac{1}{k} \mathcal{D}_{34}\right)$ contains either the singular point $P_{6}$, or the singular point $P_{7}$ by Lemma 2.3 .

Suppose that the set $\mathbb{C S}\left(U_{34}, \frac{1}{k} \mathcal{D}_{34}\right)$ contains the point $P_{7}$. Let $\zeta: U \rightarrow U_{34}$ be the weighted blow up of $P_{7}$ with weights $(1,1,3), \mathcal{H}$ be the proper transform of $\mathcal{M}$ on $U, H$ be a general surface of $\mathcal{H}$, and $D$ be a general surface of the pencil $\left|-3 K_{U}\right|$. Then $D$ is normal, and the 
base locus of the pencil $\left|-3 K_{U}\right|$ consists of the irreducible curve $Z$ such that $\alpha_{3} \circ \beta_{4}(Z)$ is the unique base curve of the pencil $\left|-3 K_{X}\right|$. Moreover, the equivalence $\left.\mathcal{H}\right|_{D} \sim_{\mathbb{Q}} k Z$ holds, and the inequality $Z^{2}<0$ holds on the surface $D$, which is impossible by Lemmas 2.8 and 2.10.

Therefore, the set $\mathbb{C} \mathbb{S}\left(U_{34}, \frac{1}{k} \mathcal{D}_{34}\right)$ contains the point $P_{6}$.

The hypersurface $X$ can be given by the quasihomogeneous equation

$$
w^{2} z+w f_{14}(x, y, z, t)+f_{24}(x, y, z, t)=0 \subset \operatorname{Proj}(\mathbb{C}[x, y, z, t, w]),
$$

where $\operatorname{wt}(x)=1, \operatorname{wt}(y)=3, \operatorname{wt}(z)=4, \operatorname{wt}(t)=7, \operatorname{wt}(w)=10$, and $f_{i}(x, y, z, w)$ is a quasihomogeneous polynomial of degree $i$. Let $\mathcal{P}$ be a pencil consisting of the surfaces that are cut on the hypersurface $X$ by the equations $\lambda x^{4}+\mu z=0$, where $(\lambda, \mu) \in \mathbb{P}^{1}$. Then the base locus of the pencil $\mathcal{P}$ consists of the irreducible curve that are cut on $X$ by the equations $x=z=0$.

Let $\pi: W \rightarrow U_{34}$ be the weighted blow up of $P_{6}$ with weights $(1,1,2), \mathcal{B}$ be the proper transform of $\mathcal{M}$ on $W, \mathcal{H}$ be the proper transform of $\mathcal{P}$ on $W, H$ be a general surface of the pencil $\mathcal{H}$, and $E, F$ and $G$ be the exceptional divisors of $\alpha_{3}, \beta_{4}$ and $\pi$ respectively. Then

$$
H \sim_{\mathbb{Q}}\left(\alpha_{3} \circ \beta_{4} \circ \pi\right)^{*}\left(-4 K_{X}\right)-\frac{1}{5}\left(\beta_{4} \circ \pi\right)^{*}(E)-\frac{4}{7}(\pi)^{*}(F)-\frac{1}{3} G,
$$

the surface $H$ normal, and the base locus of the pencil $\mathcal{H}$ consists of the curves $C$ and $L$ such that $\alpha_{3} \circ \beta_{4} \circ \pi(C)$ is the unique base curve of the pencil $\mathcal{P}$, and the curve $\beta_{4} \circ \pi(L)$ is the unique curve on the surface $E \cong \mathbb{P}(1,3,7)$ that is contained in the linear system $\left|\mathcal{O}_{\mathbb{P}(1,3,7)}(1)\right|$.

Let $S$ be the surface of the linear system $\left|-K_{W}\right|$, and $\bar{E}$ be the proper transform of the surface $E$ on the variety $W$. Then $S \cdot H=C+L$ and $\bar{E} \cdot H=4 L$, but

$$
\bar{E} \sim_{\mathbb{Q}}\left(\beta_{4} \circ \pi\right)^{*}(E)-\frac{4}{7}(\pi)^{*}(F)-\frac{1}{3} G,
$$

which implies that the intersection form of the curves $L$ and $C$ on the surface $H$ is negatively defined. The equivalence $\left.\mathcal{B}\right|_{H} \sim_{\mathbb{Q}} k C+k L$ holds, which contradicts Lemmas 2.10] and 2.8

Hence, we have $\mathbb{C} S\left(X, \frac{1}{k} \mathcal{M}\right)=\left\{P_{2}, P_{3}\right\}$, and $\mathcal{D}_{23} \sim_{\mathbb{Q}}-k K_{U_{23}}$ by Theorem 2.2

It easily follows from Lemmas 2.1] and 2.3, the proof of Lemma 36.5] and Corollary 36.4 that the set $\mathbb{C} \mathbb{S}\left(U_{23}, \frac{1}{k} \mathcal{D}_{23}\right)$ contains the singular point of the variety $U_{23}$ that is a quotient singularity of type $\frac{1}{7}(1,3,4)$ contained in the exceptional divisor of $\beta_{3}$, which implies that the proper transform of the linear system $\mathcal{M}$ on the variety $Y$ lies in the fibers of the fibration $\eta$.

37. Case $n=64$, hypersurface of Degree 26 in $\mathbb{P}(1,2,5,6,13)$.

We use the notations and assumptions of Section 3 . Let $n=64$. Then $X$ is a general hypersurface in $\mathbb{P}(1,2,5,6,13)$ of degree 26 . The singularities of the hypersurface $X$ consist of the points $P_{1}, P_{2}, P_{3}$ and $P_{4}$ that are singularities of type $\frac{1}{2}(1,1,1)$, the point $P_{5}$ that is a singularity of type $\frac{1}{5}(1,2,3)$, and the point $P_{6}$ that is a singularity of type $\frac{1}{6}(1,1,5)$.

There is a commutative diagram

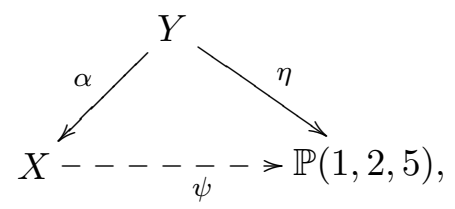

where $\psi$ is a projection, $\alpha$ is the blow up of $P_{6}$ with weights $(1,1,5)$, and $\eta$ is an elliptic fibration.

There is a commutative diagram

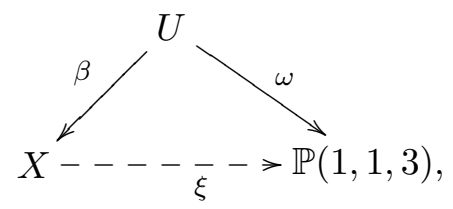

where $\xi$ is a projection, $\beta$ is the blow up of $P_{5}$ with weights $(1,2,3)$, and $\omega$ is an elliptic fibration. 
Proposition 37.1. Either there is a commutative diagram

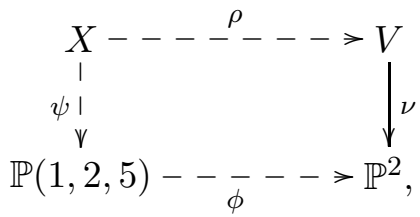

or there is a commutative diagram

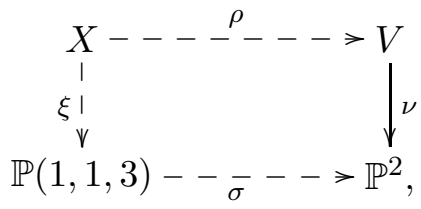

where $\phi$ and $\sigma$ are birational maps.

Proof. See the proof of Proposition 33.1

38. Case $n=65$, hypersurface of Degree 27 in $\mathbb{P}(1,2,5,9,11)$.

We use the notations and assumptions of Section 3. Let $n=65$. Then $X$ is a hypersurface of degree 27 in $\mathbb{P}(1,2,5,9,11)$, the equality $-K_{X}^{3}=3 / 110$ holds, and the singularities of $X$ consist of the point $P_{1}$ that is a quotient singularity of type $\frac{1}{2}(1,1,1)$, the point $P_{2}$ that is a quotient singularity of type $\frac{1}{5}(1,1,4)$, and the point $P_{3}$ that is a quotient singularity of type $\frac{1}{11}(1,2,9)$.

There is a commutative diagram

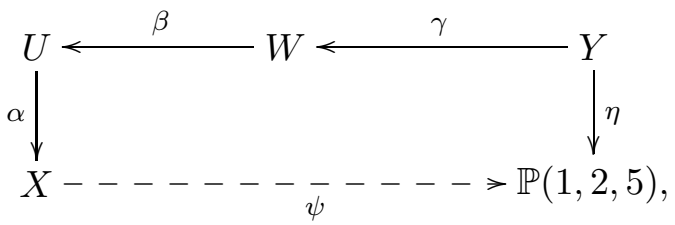

where $\psi$ is a projection, $\alpha$ is the weighted blow up of $P_{3}$ with weights $(1,2,9), \beta$ is the weighted blow up with weights $(1,2,7)$ of the point of $U$ that is a singularity of type $\frac{1}{9}(1,2,7)$ contained in the $\alpha$-exceptional divisor, $\gamma$ is the weighted blow up with weights $(1,2,5)$ of the singular point of type $\frac{1}{7}(1,2,5)$ contained in the $\beta$-exceptional divisor, and $\eta$ is an elliptic fibration.

Proposition 38.1. The claim of Theorem 1.10 holds for $n=65$.

Let us prove Proposition 38.1] It follows from Theorem [3.5. Lemma 3.14 and Proposition 3.7 that $\mathbb{C S}\left(X, \frac{1}{k} \mathcal{M}\right)=\left\{P_{3}\right\}$. Let $E$ be the exceptional divisor of $\alpha, \mathcal{D}$ be the proper transform of the linear system $\mathcal{M}$ on $U$, and $P_{4}$ and $P_{5}$ be the singular points of $U$ that are singularities of types $\frac{1}{2}(1,1,1)$ and $\frac{1}{9}(1,2,7)$ contained in $E$ respectively. Then $\mathcal{D} \sim_{\mathbb{Q}}-k K_{U}$ by Theorem 2.2

It follows from Lemmas 2.1 and 2.3 and the proof of Lemma 32.2 that $\mathbb{C S}\left(U, \frac{1}{k} \mathcal{D}\right)=\left\{P_{5}\right\}$.

Let $F$ be the exceptional divisor of the morphism $\beta, \mathcal{H}$ be the proper transform of the linear system $\mathcal{M}$ on the variety $W$, and $P_{6}$ and $P_{7}$ be the singular points of $W$ that are singularities of types $\frac{1}{2}(1,1,1)$ and $\frac{1}{7}(1,2,5)$ contained in $F$ respectively. Then $\mathcal{H} \sim_{\mathbb{Q}}-k K_{W}$ by Theorem 2.2,

It follows from Lemmas 2.1 and 2.3 that $\mathbb{C S}\left(U, \frac{1}{k} \mathcal{D}\right) \cap\left\{P_{6}, P_{7}\right\} \neq \varnothing$.

Remark 38.2. In the case when the set $\mathbb{C} \mathbb{S}\left(W, \frac{1}{k} \mathcal{H}\right)$ contains the point $P_{7}$, it follows from Theorem 2.2 that there is a commutative diagram

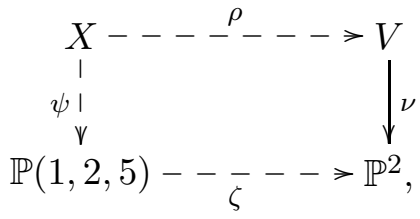

where $\zeta$ is a birational map. 
We may assume that $P_{6} \in \mathbb{C} \mathbb{S}\left(W, \frac{1}{k} \mathcal{H}\right)$. Note, that $E \cong \mathbb{P}(1,2,9)$ and $F \cong \mathbb{P}(1,2,7)$.

Let $\pi: Z \rightarrow W$ be the weighted blow up of $P_{6}$ with weights $(1,1,1), G$ be the exceptional divisor of $\pi$, and $\mathcal{B}$ be the proper transform of $\mathcal{M}$ on the variety $Z$. Then $G \cong \mathbb{P}^{2}$, and the equivalence $\mathcal{B} \sim_{\mathbb{Q}}-k K_{Z}$ holds by Theorem 2.2 .

The hypersurface $X$ can be given by equation

$$
w^{2} z+w f_{16}(x, y, z, t)+f_{27}(x, y, z, t)=0 \subset \mathbb{P}(1,2,5,9,11) \cong \operatorname{Proj}(\mathbb{C}[x, y, z, t, w]),
$$

where $\operatorname{wt}(x)=1, \operatorname{wt}(y)=2, \operatorname{wt}(z)=5, \operatorname{wt}(t)=9, \operatorname{wt}(w)=11$, and $f_{i}(x, y, z, t)$ is a quasihomogeneous polynomial of degree $i$. Let $\bar{E}$ and $\bar{F}$ be the proper transforms of the exceptional divisors $E$ and $F$ on the variety $Z$ respectively, and $\mathcal{P}$ be the proper transform on $Z$ of the pencil of surfaces that are cut out on the hypersurface $X$ by $\lambda x^{5}+\mu z=0$, where $(\lambda, \mu) \in \mathbb{P}^{1}$.

The base locus of $\mathcal{P}$ consists of the irreducible curves $C, L_{1}, L_{2}, \Delta_{1}, \Delta_{2}$ and $\Delta$ such that the curve $\alpha \circ \beta \circ \pi(C)$ is cut out on $X$ by $x=z=0$, the curve $\beta \circ \pi\left(L_{1}\right)$ is contained in the exceptional divisor $E$, the curve $\beta \circ \pi\left(L_{1}\right)$ is the unique curve in $\left|\mathcal{O}_{\mathbb{P}(1,2,9)}(1)\right|$, the curve $\pi\left(L_{1}\right)$ is contained in $F$, the curve $\pi\left(L_{1}\right)$ is contained in $\left|\mathcal{O}_{\mathbb{P}(1,2,7)}(1)\right|$, the curves $\Delta_{1}$ and $\Delta_{2}$ are the lines on $G$ that are cut out by $\bar{E}$ and $\bar{F}$ respectively, and the curve $\Delta$ is a line on $G$, which is different from the lines $\Delta_{1}$ and $\Delta_{2}$.

Let $D$ be a general surface of the pencil $\mathcal{P}$, and $S$ be the proper transform on $Z$ of the surface that is cut on the hypersurface $X$ by the equation $x=0$. Then

$$
S \cdot D=C+L_{1}+L_{2}, \bar{E} \cdot D=5 L_{1}+\Delta_{1}, \bar{F} \cdot D=5 L_{2}+\Delta_{2},
$$

the surface $D$ is normal, and $D$ is smooth in the neighborhood of $G$. In particular, it follows from the local computations and the adjunction formula that the equalities

$$
\Delta_{1} \cdot \Delta_{2}=\Delta_{1} \cdot L_{2}=\Delta_{2} \cdot L_{1}=1 \Delta_{1} \cdot C=\Delta_{2} \cdot C=0, \Delta_{1}^{2}=\Delta_{2}^{2}=-4
$$

hold on the surface $D$. However, we have

$$
\left\{\begin{array}{l}
\bar{F} \sim_{\mathbb{Q}} \pi^{*}(F)-\frac{1}{2} G, \\
\bar{E} \sim_{\mathbb{Q}}(\beta \circ \pi)^{*}(E)-\frac{7}{9} \pi^{*}(F)-\frac{1}{2} G, \\
D \sim_{\mathbb{Q}}(\alpha \circ \beta \circ \pi)^{*}\left(-5 K_{X}\right)-\frac{5}{11}(\beta \circ \pi)^{*}(E)-\frac{5}{9} \pi^{*}(F)-\frac{3}{2} G, \\
S \sim_{\mathbb{Q}}(\alpha \circ \beta \circ \pi)^{*}\left(-K_{X}\right)-\frac{1}{11}(\beta \circ \pi)^{*}(E)-\frac{1}{9} \pi^{*}(F)-\frac{1}{2} G .
\end{array}\right.
$$

It follows from the equalities 38.3 and equivalences 38.4 that the equalities

$$
C \cdot C=L_{1} \cdot L_{1}=-\frac{1}{2}, L_{2} \cdot L_{2}=-\frac{3}{7}, C \cdot L_{1}=C \cdot L_{2}=L_{1} \cdot L_{2}=0
$$

hold on $D$. The intersection form of the curves $C, L_{1}$ and $L_{2}$ on the surface $D$ is negatively defined, but $\left.\mathcal{B}\right|_{D} \sim_{\mathbb{Q}} k C+k L_{1}+k L_{2}$, which contradicts Lemmas 2.10 and 2.8.

39. Case $n=68$, hypersurface of Degree 28 IN $\mathbb{P}(1,3,4,7,14)$.

We use the notations and assumptions of Section 3. Let $n=68$. Then $X$ is a hypersurface of degree 28 in $\mathbb{P}(1,3,4,7,14)$, the singularities of the $X$ consist of the point $P_{1}$ that is a singularity of type $\frac{1}{2}(1,1,1)$, the point $P_{2}$ that is a singularity of type $\frac{1}{3}(1,1,2)$, the points $P_{3}$ and $P_{4}$ that are singularities of type $\frac{1}{7}(1,3,4)$, and $-K_{X}^{3}=1 / 42$.

Proposition 39.1. The claim of Theorem 1.10 holds for $n=68$.

Proof. It follows from Lemma 3.14 and Proposition 3.7 that $\mathbb{C S}\left(X, \frac{1}{k} \mathcal{M}\right) \subseteq\left\{P_{3}, P_{4}\right\}$, but the proof is trivial if $\mathbb{C} \mathbb{S}\left(X, \frac{1}{k} \mathcal{M}\right)=\left\{P_{3}, P_{4}\right\}$. Hence, we may assume that $P_{4} \notin \mathbb{C} \mathbb{S}\left(X, \frac{1}{k} \mathcal{M}\right)$. 
There is a commutative diagram

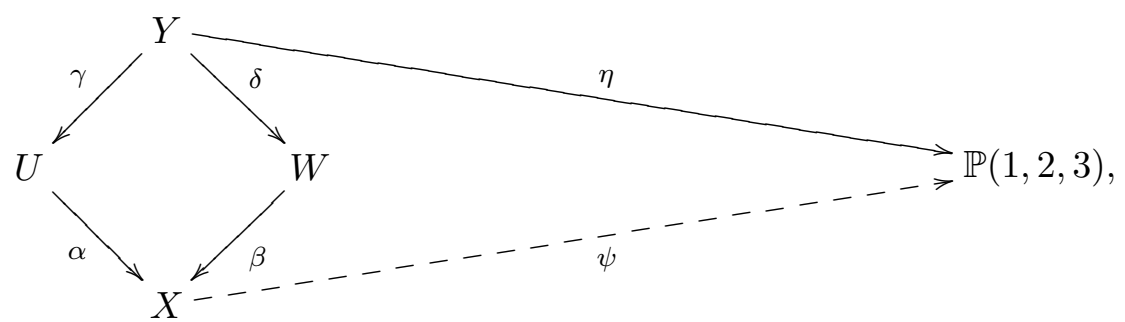

where $\psi$ is a projection, $\alpha$ is the weighted blow up of $P_{3}$ with weights $(1,3,4), \beta$ is the weighted blow up of the point $P_{4}$ with weights $(1,3,4), \gamma$ is the weighted blow up with weights $(1,3,4)$ of the proper transform of the singular point $P_{4}$ on the variety $U, \delta$ is the weighted blow up with weights $(1,3,4)$ of the proper transform of $P_{3}$ on $W$, and $\eta$ is an elliptic fibration.

Let $\mathcal{B}$ be the proper transform of $\mathcal{M}$ on $U$, and $P_{5}$ and $P_{6}$ be the singular points of $U$ that are singularities of types $\frac{1}{3}(1,1,2)$ and $\frac{1}{4}(1,1,3)$ contained in the exceptional divisor of the birational morphism $\alpha$ respectively. Then $\mathbb{C} \mathbb{S}\left(U, \frac{1}{k} \mathcal{B}\right) \cap\left\{P_{5}, P_{6}\right\} \neq \varnothing$ by Lemma 2.3 .

Suppose that $P_{6} \in \mathbb{C} S\left(U, \frac{1}{k} \mathcal{B}\right)$. Let $\pi: W \rightarrow U$ be the weighted blow up of the point $P_{6}$ with weights $(1,1,3)$, and $\mathcal{B}$ and $\mathcal{P}$ be the proper transforms of $\mathcal{M}$ and the pencil $\left|-3 K_{X}\right|$ on the variety $W$ respectively. Then $\mathcal{B} \sim_{\mathbb{Q}}-k K_{W}$ by Theorem 2.2. and the base locus of $\mathcal{P}$ consists of the irreducible curve $Z$ such that $\alpha \circ \pi(Z)$ is the base curve of the pencil $\left|-3 K_{X}\right|$.

Let $S$ be a general surface in $\mathcal{P}$, and $B$ be a general surface in $\mathcal{B}$. Then the surface $S$ is normal, the inequality $Z^{2}<0$ holds on the surface $S$, but $\left.\mathcal{B}\right|_{S} \sim_{\mathbb{Q}} k Z$. Therefore, the support of the cycle $B \cdot S$ is contained in $Z$ by Lemma 2.10, which is impossible by Lemma 2.8 .

Hence, the set $\mathbb{C} \mathbb{S}\left(U, \frac{1}{k} \mathcal{B}\right)$ contains the point $P_{5}$.

Let $\zeta: Z \rightarrow U$ be the weighted blow up of $P_{5}$ with weights $(1,1,2)$, and $\mathcal{D}$ and $\mathcal{H}$ be the proper transforms of $\mathcal{M}$ and $\left|-4 K_{X}\right|$ on $Z$ respectively. Then $\mathcal{D} \sim_{\mathbb{Q}}-k K_{Z}$ by Theorem 2.2 . and the base locus of $\mathcal{H}$ consists of a curve $C$ such that $\alpha \circ \zeta(C)$ is the base curve of $\left|-4 K_{X}\right|$.

Let $H$ be a general surface in $\mathcal{H}$. Then $H \cdot C=0$ and $H^{3}>0$. Thus, the divisor $H$ is nef and big. On the other hand, the equality $H \cdot D_{1} \cdot D_{2}=0$ holds, where $D_{1}$ and $D_{2}$ are general surfaces of the linear system $\mathcal{D}$, which is impossible by Corollary 2.7.

40. Case $n=74$, hypersurface of Degree 30 in $\mathbb{P}(1,3,4,10,13)$.

We use the notations and assumptions of Section 3 Let $n=74$. Then $X$ is a hypersurface of degree 30 in $\mathbb{P}(1,3,4,10,13)$, the equality $-K_{X}^{3}=1 / 52$ holds, and the singularities of $X$ consist of the point $P_{1}$ that is a quotient singularity of type $\frac{1}{2}(1,1,1)$, the point $P_{2}$ that is a quotient singularity of type $\frac{1}{4}(1,1,3)$, and the point $P_{3}$ that is a quotient singularity of type $\frac{1}{13}(1,3,10)$.

There is a commutative diagram

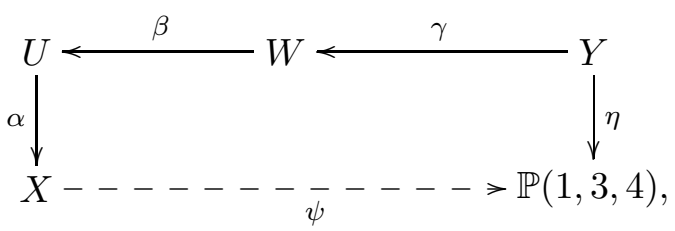

where $\psi$ is a projection, $\alpha$ is the weighted blow up of $P_{3}$ with weights $(1,3,10), \beta$ is the weighted blow up with weights $(1,3,7)$ of the singular point of the variety $U$ that is a quotient singularity of type $\frac{1}{10}(1,3,7)$ contained in the exceptional divisor of the morphism $\alpha, \gamma$ is the weighted blow up with weights $(1,3,4)$ of the singular point of the variety $W$ that is a quotient singularity of type $\frac{1}{7}(1,3,4)$ contained in the exceptional divisor of $\beta$, and $\eta$ is an elliptic fibration.

In the rest of the section we prove the following result.

Proposition 40.1. The claim of Theorem 1.10 holds for $n=74$.

It follows from Theorem 3.5. Lemma 3.14 and Proposition 3.7 $\mathbb{C S}\left(X, \frac{1}{k} \mathcal{M}\right)=\left\{P_{3}\right\}$.

Let $E$ be the exceptional divisor of the morphism $\alpha, \mathcal{D}$ be the proper transform of the linear system $\mathcal{M}$ on the variety $U$, and $P_{4}$ and $P_{5}$ be the singular points of $U$ that are quotient 
singularities of types $\frac{1}{3}(1,1,2)$ and $\frac{1}{10}(1,3,7)$ contained in the divisor $E$ respectively. Then the equivalence $\mathcal{D} \sim_{\mathbb{Q}}-k K_{U}$ holds by Theorem 2.2 .

Lemma 40.2. The set $\mathbb{C S}\left(U, \frac{1}{k} \mathcal{D}\right)$ does not contain the point $P_{4}$.

Proof. Suppose that the set $\mathbb{C} S\left(U, \frac{1}{k} \mathcal{D}\right)$ contains the point $P_{4}$. Let $\pi: Z \rightarrow U$ be the weighted blow up of $P_{4}$ with weights $(1,1,2), G$ be the exceptional divisor of $\pi$, and $\mathcal{B}$ be the proper transforms of the linear system $\mathcal{M}$ on the variety $Z$. Then $\mathcal{B} \sim_{\mathbb{Q}}-k K_{Z}$ by Theorem 2.2.

Let $D$ be a divisor on $Z$ such that the equivalence

$$
D \sim_{\mathbb{Q}}-4 K_{Z}-\pi^{*}\left(36 K_{U}\right)
$$

holds. Analyzing the base locus of the pencil $\left|-4 K_{Z}\right|$, we see that $D$ is nef and big, but

$$
D \cdot B_{1} \cdot B_{2}=\left((\alpha \circ \pi)^{*}\left(-k K_{X}\right)-\frac{k}{13} \pi^{*}(E)-\frac{k}{3} G\right)^{2}\left(-4 K_{Z}-\pi^{*}\left(36 K_{U}\right)\right)=0,
$$

where $B_{1}$ and $B_{2}$ are general surfaces in $\mathcal{B}$. The latter is impossible by Corollary 2.7

Corollary 40.3. The set $\mathbb{C S}\left(U, \frac{1}{k} \mathcal{D}\right)$ consists of the point $P_{5}$ by Lemmas 2.1 and 2.3.

Let $F$ be the exceptional divisor of the morphism $\beta, \mathcal{H}$ be the proper transform of the linear system $\mathcal{M}$ on the variety $W$, and $P_{6}$ and $P_{7}$ be the singular points of $W$ that are quotient singularities of types $\frac{1}{3}(1,1,2)$ and $\frac{1}{7}(1,3,4)$ contained in $F$ respectively. Then $\mathcal{H} \sim_{\mathbb{Q}}-k K_{W}$.

Suppose that the set $\mathbb{C S}\left(W, \frac{1}{k} \mathcal{H}\right)$ contains the point $P_{7}$. Then it easily follows from the proof of Lemma 32.3 that the claim of Theorem 1.10 holds for the hypersurface $X$. Hence, we may assume that the set $\mathbb{C S}\left(W, \frac{1}{k} \mathcal{H}\right)$ does not contain the point $P_{7}$. Thus, the set $\mathbb{C} \mathbb{S}\left(W, \frac{1}{k} \mathcal{H}\right)$ consists of the singular point $P_{6}$ by Lemmas 2.1 and 2.3

Let $\pi: Z \rightarrow W$ be the weighted blow up of the point $P_{6}$ with weights $(1,1,2), G$ be the exceptional divisor of the birational morphism $\pi$, and $\mathcal{B}$ be the proper transform of the linear system $\mathcal{M}$ on the variety $Z$. Then $\mathcal{B} \sim_{\mathbb{Q}}-k K_{Z}$ by Theorem 2.2

Let $D$ be a divisor on $Z$ such that the equivalence

$$
D \sim_{\mathbb{Q}}-4 K_{Z}-(\beta \circ \pi)^{*}\left(20 K_{U}\right)-\pi^{*}\left(24 K_{W}\right)
$$

holds. The divisor $D$ is nef and big, but $D \cdot B_{1} \cdot B_{2}=0$, where $B_{1}$ and $B_{2}$ are general surfaces of the linear system $\mathcal{B}$, which is impossible by Corollary 2.7

\section{Case $n=79$, hypersurface of Degree 33 in $\mathbb{P}(1,3,5,11,14)$.}

We use the notations and assumptions of Section 3 Let $n=79$. Then $X$ is a hypersurface of degree 33 in $\mathbb{P}(1,3,5,11,14)$, the equality $-K_{X}^{3}=1 / 70$ holds, and the singularities of $X$ consist of the points $P_{1}$ and $P_{2}$ that are singularities of type $\frac{1}{5}(1,1,4)$ and $\frac{1}{14}(1,3,11)$ respectively.

Proposition 41.1. The claim of Theorem 1.10 holds for $n=79$.

Proof. There is a commutative diagram

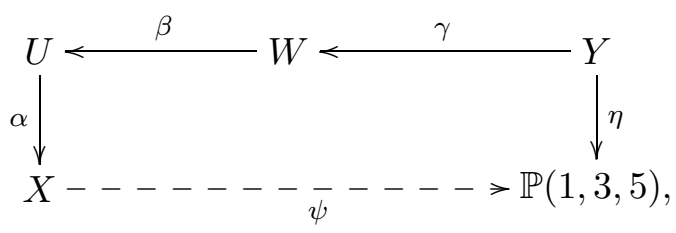

where $\psi$ is a projection, $\alpha$ is the weighted blow up of $P_{2}$ with weights $(1,3,11), \beta$ is the weighted blow up with weights $(1,3,8)$ of the singular point of type $\frac{1}{11}(1,3,8)$ contained in the exceptional divisor of $\alpha, \gamma$ is the weighted blow up with weights $(1,3,5)$ of the singular point of type $\frac{1}{8}(1,3,5)$ contained in the exceptional divisor of $\beta$, and $\eta$ is an elliptic fibration.

It follows from Theorem 3.5, Lemma 3.14 and Proposition 3.7 that $\mathbb{C S}\left(X, \frac{1}{k} \mathcal{M}\right)=\left\{P_{2}\right\}$.

Let $E$ be the exceptional divisor of the morphism $\alpha, \mathcal{D}$ be the proper transform of the linear system $\mathcal{M}$ on $U$, and $P_{3}$ and $P_{4}$ be the singular points of $U$ that are quotient singularities of types $\frac{1}{3}(1,1,2)$ and $\frac{1}{11}(1,3,8)$ contained in $E$ respectively. Then $\mathcal{D} \sim_{\mathbb{Q}}-k K_{U}$, and it follows from Lemmas 2.1 and 2.3 and the proof of Lemma 40.2 that $\mathbb{C} S\left(U, \frac{1}{k} \mathcal{D}\right)=\left\{P_{4}\right\}$. 
Let $F$ be the exceptional divisor of the morphism $\beta, \mathcal{H}$ be the proper transform of the linear system $\mathcal{M}$ on the variety $W$, and $P_{5}$ and $P_{6}$ be the singular points of $W$ that are quotient singularities of types $\frac{1}{3}(1,1,2)$ and $\frac{1}{8}(1,3,4)$ contained in $F$. Then $\mathcal{H} \sim_{\mathbb{Q}}-k K_{W}$.

In the case when the set $\mathbb{C S}\left(W, \frac{1}{k} \mathcal{H}\right)$ contains the point $P_{6}$, it easily follows from Theorem 2.2 that the claim of Theorem 1.10 holds for the hypersurface $X$. Therefore, we may assume that the set $\mathbb{C S}\left(W, \frac{1}{k} \mathcal{H}\right)$ consists of the point $P_{5}$ by Lemmas 2.1 and 2.3

Let $\pi: Z \rightarrow W$ be the weighted blow up of t $P_{5}$ with weights $(1,1,2), G$ be the exceptional divisor of $\pi$, and $\mathcal{B}$ be the proper transform of $\mathcal{M}$ on $Z$. Then $\mathcal{B} \sim_{\mathbb{Q}}-k K_{Z}$ by Theorem 2.2 .

The hypersurface $X$ can be given by the equation

$$
w^{2} z+w f_{19}(x, y, z, t)+f_{33}(x, y, z, t)=0 \subset \mathbb{P}(1,2,3,8,11) \cong \operatorname{Proj}(\mathbb{C}[x, y, z, t, w]),
$$

where $\operatorname{wt}(x)=1, \operatorname{wt}(y)=3, \operatorname{wt}(z)=5, \operatorname{wt}(t)=11, \operatorname{wt}(w)=14$, and $f_{i}(x, y, z, t)$ is a quasihomogeneous polynomial of degree $i$. Let $\mathcal{P}$ be the linear system on the hypersurface $X$ that is generated by the monomials $x^{30}, y^{10}, z^{6}, t^{2} x^{8}, t^{2} y^{2} x^{2}, t y^{6} x$ and $w t z, \mathcal{R}$ be the proper transform of $\mathcal{P}$ on the variety $Z$, and $R$ be a general surface in $\mathcal{R}$. Then $R$ is nef and big, but

$$
R \sim_{\mathbb{Q}}(\alpha \circ \beta \circ \pi)^{*}\left(-30 K_{X}\right)-\frac{30}{11}(\beta \circ \pi)^{*}(E)-\frac{8}{11} \pi^{*}(F)-\frac{2}{3} G,
$$

which implies that the equality $R \cdot B_{1} \cdot B_{2}=0$ holds, where $B_{1}$ and $B_{2}$ are general surfaces of the linear system $\mathcal{B}$. The latter contradicts Corollary 2.7

\section{Case $n=80$, hypersurface of Degree 34 in $\mathbb{P}(1,3,4,10,17)$.}

We use the notations and assumptions of Section 3 Let $n=80$. Then $X$ is a hypersurface of degree 34 in $\mathbb{P}(1,3,4,10,17)$, whose singularities consist of the point $P_{1}$ that is a singularity of type $\frac{1}{2}(1,1,1)$, the point $P_{2}$ that is a singularity of type $\frac{1}{3}(1,1,2)$, the point $P_{3}$ that is a singularity of type $\frac{1}{4}(1,1,3)$, and the point $P_{4}$ that is a singularity of type $\frac{1}{10}(1,3,7)$.

Proposition 42.1. The claim of Theorem 1.10 holds for $n=80$.

Proof. It follows from Proposition 3.7 that $\mathbb{C S}\left(X, \frac{1}{k} \mathcal{M}\right)=\left\{P_{4}\right\}$. There is a commutative diagram

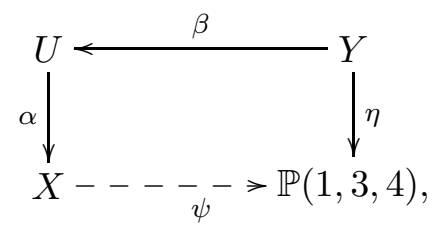

where $\psi$ is a projection, $\alpha$ is the weighted blow up of $P_{4}$ with weights $(1,3,7), \beta$ is the weighted blow up with weights $(1,3,4)$ of the singular point the variety $U$ that is a quotient singularity of type $\frac{1}{7}(1,3,4)$ contained in the exceptional divisor of $\alpha$, and $\eta$ is an elliptic fibration.

Let $\mathcal{D}$ be the proper transform of the linear system $\mathcal{M}$ on the variety $U$, and $P_{5}$ and $P_{6}$ be the singular points of the variety $U$ that are quotient singularities of types $\frac{1}{3}(1,1,2)$ and $\frac{1}{4}(1,1,3)$ contained in the exceptional divisor of $\alpha$ respectively. Then the equivalence $\mathcal{D} \sim_{\mathbb{Q}}-k K_{U}$.

It follows from Lemmas 2.1 and 2.3 that either the set $\mathbb{C S}\left(U, \frac{1}{k} \mathcal{D}\right)$ contains the point $P_{5}$, or the set $\mathbb{C S}\left(U, \frac{1}{k} \mathcal{D}\right)$ contains the point $P_{6}$. In the latter case it follows from Theorem 2.2 that the claim of Theorem 1.10 holds for $X$. Therefore, we may assume $P_{5} \in \mathbb{C} \mathbb{S}\left(U, \frac{1}{k} \mathcal{D}\right)$.

The hypersurface $X$ can be given by the quasihomogeneous equation

$$
t^{3} z+t^{2} f_{14}(x, y, z, w)+t f_{24}(x, y, z, w)+f_{34}(x, y, z, w)=0 \subset \operatorname{Proj}(\mathbb{C}[x, y, z, t, w]),
$$

where $\operatorname{wt}(x)=1, \operatorname{wt}(y)=3, \operatorname{wt}(z)=4, \operatorname{wt}(t)=10, \operatorname{wt}(w)=17$, and $f_{i}(x, y, z, w)$ is a general quasihomogeneous polynomial of degree $i$. Let $\mathcal{P}$ be a pencil consisting of the surfaces that are cut on $X$ by the equations $\lambda x^{4}+\mu z=0$, where $(\lambda, \mu) \in \mathbb{P}^{1}$. Then the base locus of $\mathcal{P}$ consists of the irreducible curve that is cut on $X$ by the equations $x=z=0$.

Let $\gamma: W \rightarrow U$ be the weighted blow up of $P_{5}$ with weights $(1,1,2), \mathcal{B}$ be the proper transform of the linear system $\mathcal{M}$ on the variety $W, \mathcal{H}$ be the proper transform of the pencil $\mathcal{P}$ on the 
variety $W, D$ be a sufficiently general surface of the pencil $\mathcal{H}$, and $E$ and $F$ be the exceptional divisors of the morphisms $\alpha$ and $\gamma$ respectively. Then the surface $D$ is normal, the equivalences

$$
D \sim_{\mathbb{Q}}-4 K_{W} \sim_{\mathbb{Q}}(\alpha \circ \gamma)^{*}\left(-4 K_{X}\right)-\frac{2}{5} \gamma^{*}(E)-\frac{4}{3} F
$$

hold, and the base locus of the pencil $\mathcal{H}$ consists of the curves $C$ and $L$ such that $\alpha \circ \gamma(C)$ is the base curve of the pencil $\mathcal{P}$, and $\gamma(L)$ is the curve on the surface $E \cong \mathbb{P}(1,3,7)$ that is contained in the linear system $\left|\mathcal{O}_{\mathbb{P}(1,3,7)}(1)\right|$.

Let $S$ be the surface of the linear system $\left|-K_{W}\right|$, and $\bar{E}$ be the proper transform of the surface $E$ on the variety $W$. Then $S \cdot D=C+L$ and $\bar{E} \cdot D=4 L$, which implies that

$$
C \cdot C=-\frac{1}{3}, C \cdot L=0, L \cdot L=-\frac{2}{7}
$$

on the surface $D$. Hence, the intersection form of the curves $C$ and $L$ on the surface $D$ is negatively defined, but $\left.\mathcal{B}\right|_{D} \sim_{\mathbb{Q}} k C+k L$, which contradicts Lemmas 2.10 and 2.8 ,

43. Case $n=82$, hypersurface of Degree 36 in $\mathbb{P}(1,1,5,12,18)$.

We use the notations and assumptions of Section 3. Let $n=82$. Then $X$ is a hypersurface of degree 36 in $\mathbb{P}(1,1,5,12,18)$, the equality $-K_{X}^{3}=1 / 30$ holds, and the singularities of $X$ consist of the point $P_{1}$ that is a quotient singularity of type $\frac{1}{5}(1,2,3)$, and the point $P_{2}$ that is a quotient singularity of type $\frac{1}{6}(1,1,5)$. There is a commutative diagram

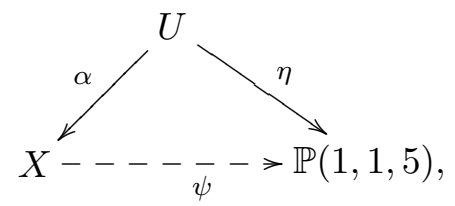

where $\psi$ is a projection, $\alpha$ is the weighted blow up of $P_{2}$ with weights $(1,1,6)$, and $\eta$ is an elliptic fibration. The hypersurface $X$ is birationally superrigid.

Proposition 43.1. The claim of Theorem 1.10 holds for $n=82$.

Proof. Suppose that $P_{2} \in \mathbb{C S}\left(X, \frac{1}{k} \mathcal{M}\right)$. Then Theorem 2.2 implies the existence of the commutative diagram

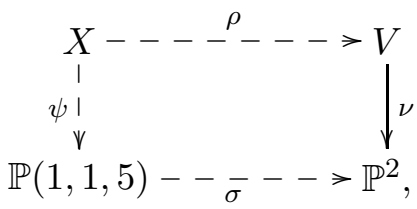

where $\sigma$ is a birational map. Thus, it follows from Theorem 3.5 Lemma 3.14 and Proposition 3.7 that we may assume that the set $\mathbb{C} \mathbb{S}\left(X, \frac{1}{k} \mathcal{M}\right)$ consists of the point $P_{1}$.

Let $\pi: W \rightarrow X$ be the weighted blow up of $P_{1}$ with weights $(1,2,3)$, and $\mathcal{B}$ be the proper transform of $\mathcal{M}$ on $W$. Then $\mathcal{B} \sim_{\mathbb{Q}}-k K_{W}$ by Theorem [2.2, and the singularities of the exceptional divisor of the morphism $\pi$ consist of the points $Q$ and $O$ that are quotient singularities of types $\frac{1}{2}(1,1,1)$ and $\frac{1}{3}(1,1,2)$ on the variety $W$ respectively.

Suppose that the set $\mathbb{C} \mathbb{S}\left(W, \frac{1}{k} \mathcal{B}\right)$ is not empty. Then the set $\mathbb{C} \mathbb{S}\left(W, \frac{1}{k} \mathcal{B}\right)$ contains either the point $O$, or the point $Q$. On the other hand, it easily follows from the proof of Proposition 22.1 that the set $\mathbb{C} S\left(W, \frac{1}{k} \mathcal{B}\right)$ does not contain neither the point $O$, nor the point $Q$, which implies that the singularities of the log pair $\left(W, \frac{1}{k} \mathcal{B}\right)$ are terminal.

It follows from the proof of Proposition 29.1 that there is a birational map $\gamma: W \rightarrow Y$ such that $\gamma$ is an antiflip, the divisor $-K_{Y}$ is nef and big, and the linear system $\left|-r K_{Y}\right|$ induced a birational map $Y \rightarrow X^{\prime}$ such that $X^{\prime}$ is a hypersurface in $\mathbb{P}(1,1,6,14,21)$ of degree 42 with canonical singularities (see the proof of Theorem 5.5.1 in [6]), where $r \gg 0$.

Let $\mathcal{H}$ be the proper transform of $\mathcal{M}$ on the variety $Y$. Then $\mathcal{H} \sim_{\mathbb{Q}}-k K_{Y}$, because $\gamma$ is an isomorphism in codimension one. The map $\gamma$ is a $\log$ flip with respect to $(W, \lambda \mathcal{B})$ for some rational number $\lambda>1 / k$. Thus, the singularities of the mobile log pair $\left(Y, \frac{1}{k} \mathcal{H}\right)$ are terminal, which contradicts Lemma 2.1. 
44. CASE $n \in\{21,24,33,35,41,42,46,50,54,55,61,62,63,67,69,71,76,77,83,85,91\}$.

We use the notations and assumptions of Section 3 .

Proposition 44.1. Suppose that $n \in\{21,24,33,35,41,42,46,50,54,55,61,62,63,67,69,71,76$, $77,83,85,91\}$. Then there is a commutative diagram

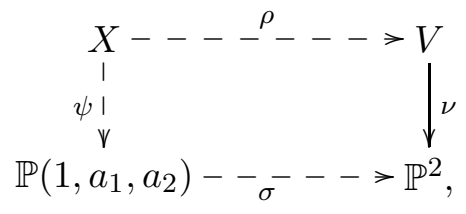

where $\psi$ is the natural projection, and $\sigma$ is a birational map.

Proof. It follows from Theorem 3.5 and Lemma 3.14 that the set $\mathbb{C S}\left(X, \frac{1}{k} \mathcal{M}\right)$ consists of singular points of the hypersurface $X$. We prove the existence of the diagram 44.2 case by case.

Case $n=21$.

The variety $X$ is a hypersurface in $\mathbb{P}(1,1,2,4,7)$ of degree 14 , the equality $-K_{X}^{3}=1 / 4$ holds, and the singularities of the hypersurface $X$ consist of the points $O_{1}$ and $O_{2}$ that are quotient singularities of type $\frac{1}{2}(1,1,1)$, and the point $P$ that is a quotient singularity of type $\frac{1}{4}(1,1,3)$.

There is a commutative diagram

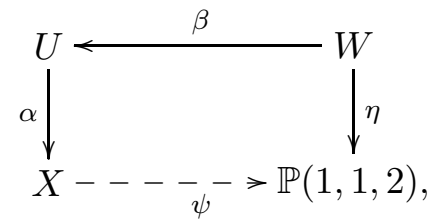

where $\psi$ is a projection, $\alpha$ is the weighted blow up of $P$ with weights $(1,1,3), \beta$ is the weighted blow up with weights $(1,1,2)$ of the singular point of $U$ that is a singularity of type $\frac{1}{3}(1,1,2)$, and $\eta$ is an elliptic fibration. It follows from Proposition 3.7 that $\mathbb{C} S\left(X, \frac{1}{k} \mathcal{M}\right)=\{P\}$.

Let $\mathcal{D}$ be the proper transform of $\mathcal{M}$ on $U$. Then $\mathcal{D} \sim_{\mathbb{Q}}-k K_{U}$ by Theorem 2.2 and it follows from Lemmas 2.1 and 2.3 that the set $\mathbb{C S}\left(U, \frac{1}{k} \mathcal{D}\right)$ contains the singular point of $U$ that is contained in the exceptional divisor of the morphism $\alpha$.

Let $\mathcal{B}$ be the proper transform of $\mathcal{M}$ on $Y$. Then $\mathcal{B} \sim_{\mathbb{Q}}-k K_{Y}$ by Theorem 2.2 which implies the existence of the the commutative diagram 44.2 .

Case $n=24$.

The variety $X$ is a hypersurface in $\mathbb{P}(1,1,2,5,7)$ of degree 15 , the equality $-K_{X}^{3}=3 / 14$ holds, and the singularities of the hypersurface $X$ consist of the point $P_{1}$ that is a quotient singularity of type $\frac{1}{2}(1,1,1)$, and the point $P_{2}$ that is a quotient singularity of type $\frac{1}{7}(1,2,5)$.

It follows from Proposition 3.7 that $\mathbb{C S}\left(X, \frac{1}{k} \mathcal{M}\right)=\left\{P_{2}\right\}$. There is a commutative diagram

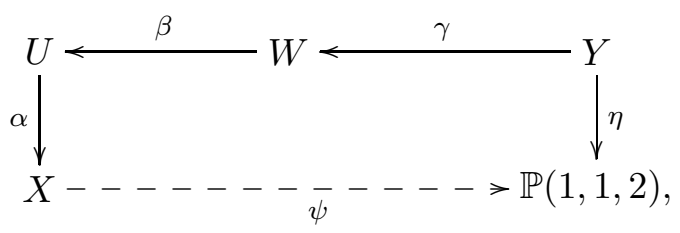

where $\alpha$ is the weighted blow up of the point $P$ with weights $(1,2,5), \beta$ is the weighted blow up with weights $(1,2,3)$ of the singular point of $U$ that is a singularity of type $\frac{1}{5}(1,2,3), \gamma$ is the weighted blow up with weights $(1,1,2)$ of the singular point of the variety $W$ that is a quotient singularity of type $\frac{1}{3}(1,1,2)$, and $\eta$ is an elliptic fibration.

The proof of Proposition 22.1 implies that $\mathcal{D} \sim_{\mathbb{Q}}-k K_{W}$, where $\mathcal{D}$ is the proper transform of the linear system $\mathcal{M}$ on the variety $W$, and the set $\mathbb{C S}\left(W, \frac{1}{k} \mathcal{D}\right)$ does not contain subvarieties of the variety $W$ that are not contained in the exceptional divisor of the morphism $\beta$.

We can apply arguments of the proof of Proposition 22.1 to the log pair $\left(W, \frac{1}{k} \mathcal{D}\right)$ to prove that the set $\mathbb{C} S\left(W, \frac{1}{k} \mathcal{D}\right)$ contains the singular point of $W$ that is a singularity of type $\frac{1}{3}(1,1,2)$, which implies the existence of the commutative diagram 44.2 by Theorem 2.2 
Case $n=33$.

The variety $X$ is a hypersurface in $\mathbb{P}(1,2,3,5,7)$ of degree 17 , the singularities of $X$ consist of the point $P_{1}$ that is a quotient singularity of type $\frac{1}{2}(1,1,1)$, the point $P_{2}$ that is a quotient singularity of type $\frac{1}{3}(1,1,2)$, the point $P_{3}$ that is a quotient singularity of type $\frac{1}{5}(1,2,3)$, and the point $P_{4}$ that is a singularity of type $\frac{1}{7}(1,2,5)$. There is a commutative diagram

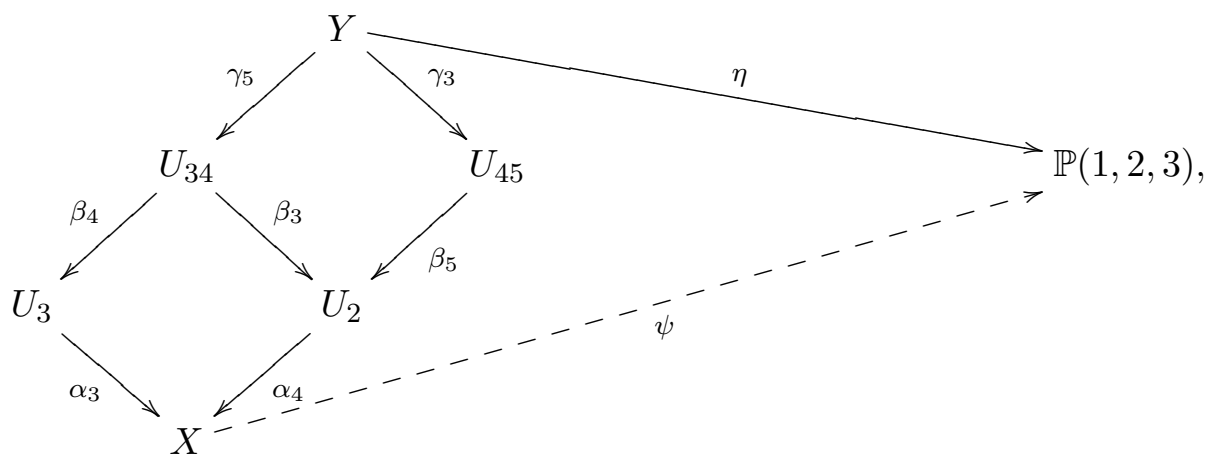

where $\alpha_{3}$ is the weighted blow up of the point $P_{3}$ with weights $(1,2,3), \alpha_{4}$ is the weighted blow up of the point $P_{4}$ with weights $(1,2,5), \beta_{4}$ is the weighted blow up with weights $(1,2,5)$ of the proper transform of $P_{4}$ on $U_{3}, \beta_{3}$ is the weighted blow up with weights $(1,2,3)$ of the proper transform of $P_{3}$ on $U_{4}, \beta_{5}$ is the weighted blow up with weights $(1,2,3)$ of the singular point of the variety $U_{4}$ that is a quotient singularity of type $\frac{1}{5}(1,2,3)$ contained in the exceptional divisor of the morphism $\alpha_{4}, \gamma_{3}$ is the weighted blow up with weights $(1,2,3)$ of the proper transform of the point $P_{3}$ on the variety $U_{45}, \gamma_{5}$ is the weighted blow up with weights $(1,2,3)$ of the singular point of $U_{34}$ that is a quotient singularity of type $\frac{1}{5}(1,2,3)$ contained in the exceptional divisor of the morphism $\beta_{4}$, and $\eta$ is an elliptic fibration.

It follows from Proposition 3.7 and the proof of Proposition 18.1 that $\mathbb{C S}\left(X, \frac{1}{k} \mathcal{M}\right)=\left\{P_{3}, P_{4}\right\}$.

Let $\mathcal{D}_{34}$ be the proper transform of $\mathcal{M}$ on $U_{34}$, and $\bar{P}_{5}$ and $\bar{P}_{6}$ be the singular points of the variety $U_{34}$ that are quotient singularities of types $\frac{1}{5}(1,2,3)$ and $\frac{1}{2}(1,1,1)$ contained in the exceptional divisor of the morphism $\beta_{4}$ respectively. Then $\mathcal{D}_{34} \sim_{\mathbb{Q}}-k K_{U_{34}}$ by Theorem [2.2.

It follows from Lemma 2.3 and the proof of Proposition 18.1 that $\bar{P}_{5} \in \mathbb{C} \mathbb{S}\left(U_{34}, \frac{1}{k} \mathcal{D}_{34}\right)$, and the existence of the commutative diagram 44.2 follows from Theorem 2.2

Case $n=35$.

The variety $X$ is a hypersurface in $\mathbb{P}(1,1,3,5,9)$ of degree 18 , whose singularities consist of the points $P_{1}$ and $P_{2}$ that are singularities of type $\frac{1}{3}(1,1,2)$, and the point $P_{3}$ that is a singularity of type $\frac{1}{5}(1,1,4)$. There is a commutative diagram

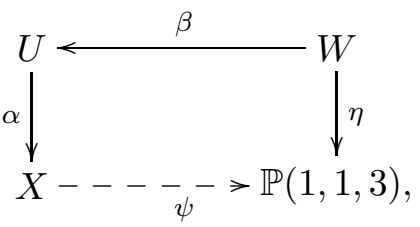

where $\alpha$ is the blow up of $P_{3}$ with weights $(1,1,4), \beta$ is the blow up with weights $(1,1,3)$ of the point of $U$ that is singularity of type $\frac{1}{4}(1,1,3)$, and $\eta$ is an elliptic fibration.

It follows from Proposition 3.7 that $\mathbb{C S}\left(X, \frac{1}{k} \mathcal{M}\right)=\left\{P_{3}\right\}$.

Let $\mathcal{D}$ is the proper transform $\mathcal{M}$ on $U$. Then $\mathcal{D} \sim_{\mathbb{Q}}-k K_{U}$ by Theorem 2.2 and it follows from Lemma 2.3 that $\mathbb{C} \mathbb{S}\left(U, \frac{1}{k} \mathcal{D}\right)$ contains the singular point of $U$ that is contained in the exceptional divisor of $\alpha$. The existence of the diagram 44.2 follows from Theorem 2.2

Case $n=41$.

The variety $X$ is a hypersurface in $\mathbb{P}(1,1,4,5,10)$ of degree 20 , whose singularitiesconsist of the point $O$ that is a singularity of type $\frac{1}{2}(1,1,1)$, the points $P_{1}$ and $P_{2}$ of type $\frac{1}{5}(1,1,4)$.

The indeterminacies of $\psi$ are resolved by blowing up $P_{1}$ and $P_{2}$ with weights $(1,1,4)$, it follows from Proposition 3.7 that $\mathbb{C S}\left(X, \frac{1}{k} \mathcal{M}\right) \subseteq\left\{P_{1}, P_{2}\right\}$, and the existence of the diagram 44.2 follows from the proof of Proposition 13.1 
Case $n=42$.

The variety $X$ is a hypersurface in $\mathbb{P}(1,2,3,5,10)$ of degree 20 , the singularities of $X$ consist of the points $P_{1}, P_{2}$ and $P_{3}$ that are quotient singularities of type $\frac{1}{2}(1,1,1)$, the point $P_{4}$ that is a singularity of type $\frac{1}{3}(1,1,2)$, the points $P_{5}$ and $P_{6}$, that are singularities of types $\frac{1}{5}(1,2,3)$.

The indeterminacies of $\psi$ are resolved by blowing up $P_{5}$ and $P_{6}$ with weights $(1,2,3)$,

It follows from Proposition 3.7 that $\mathbb{C} S\left(X, \frac{1}{k} \mathcal{M}\right) \subseteq\left\{P_{5}, P_{6}\right\}$, but the existence of the diagram 44.2 is obvious if $\mathbb{C} \mathbb{S}\left(X, \frac{1}{k} \mathcal{M}\right)=\left\{P_{5}, P_{6}\right\}$. We may assume that $\mathbb{C} \mathbb{S}\left(X, \frac{1}{k} \mathcal{M}\right)=\left\{P_{5}\right\}$.

Let $\alpha: U \rightarrow X$ be the weighted blow up of the point $P_{5}$ with weights $(1,2,3), \mathcal{B}$ be the proper transform of the linear system $\mathcal{M}$ on $U$, and $O$ and $Q$ be the singular points of $U$ that are quotient singularities of types $\frac{1}{3}(1,1,2)$ and $\frac{1}{2}(1,1,1)$ contained in the exceptional divisor of the morphism $\alpha$ respectively. Then it follows from the proof of Lemma 21.3 that $\mathbb{C} S\left(U, \frac{1}{k} \mathcal{B}\right)=\{Q\}$.

Let $\zeta: Z \rightarrow U$ be the weighted blow up of the point $Q$ with weights $(1,1,1), \mathcal{D}$ be the proper transform of the linear system $\mathcal{M}$ on the variety $Z$, and $\mathcal{H}$ be the proper transform of the linear system $\left|-3 K_{X}\right|$ on the variety $Z$. Then $\mathcal{D} \sim_{\mathbb{Q}}-k K_{Z}$ by Theorem 2.2 and the base locus of the linear system $\mathcal{H}$ consists of a curve $C$ such that $\alpha \circ \zeta(C)$ is the base curve of $\left|-3 K_{X}\right|$.

Let $S$ be a general surface in $\mathcal{H}$. Then $S$ is normal, and the inequality $C^{2}<0$ holds on the surface $S$, but the equivalence $\left.\mathcal{D}\right|_{S} \sim_{\mathbb{Q}} k C$ holds, which contradicts Lemmas 2.8 and 2.10$]$

Case $n=45$.

The variety $X$ is a hypersurface in $\mathbb{P}(1,3,4,5,8)$ of degree 20 , whose singularities consist of the point $P_{1}$ of type $\frac{1}{3}(1,1,2)$, the points $P_{2}$ and $P_{3}$ of type $\frac{1}{4}(1,1,3)$, the point $P_{4}$ of type $\frac{1}{8}(1,3,5)$.

There is a commutative diagram

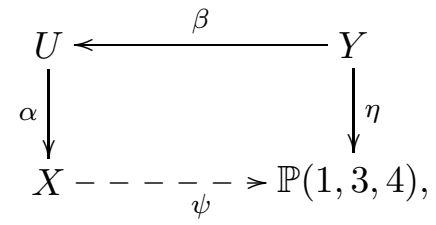

where $\alpha$ is the weighted blow up of the point $P_{4}$ with weights $(1,3,5), \beta$ is the weighted blow up with weights $(1,2,3)$ of the singular point of $U$ that is a quotient singularity of type $\frac{1}{5}(1,2,3)$ contained in the exceptional divisor of the morphism $\alpha$, and $\eta$ is an elliptic fibration.

It follows from Proposition 3.7 that $\mathbb{C S}\left(X, \frac{1}{k} \mathcal{M}\right)=\left\{P_{4}\right\}$.

Let $\mathcal{B}$ be the proper transform of the linear system $\mathcal{M}$ on the variety $U, \bar{P}_{5}$ be the singular point of the variety $U$ that is a quotient singularity of type $\frac{1}{5}(1,2,3)$ contained in the exceptional divisor of the morphism $\alpha$, and $\bar{P}_{6}$ be the singular point of the the variety $U$ that is a quotient singularity of type $\frac{1}{2}(1,1,1)$ contained in the exceptional divisor of the morphism $\alpha$.

It follows from Lemma 2.3 that $\mathbb{C S}\left(U, \frac{1}{k} \mathcal{B}\right) \cap\left\{\bar{P}_{5}, \bar{P}_{6}\right\} \neq \varnothing$, but the proof of Proposition 30.1 easily implies that $\bar{P}_{5} \in \mathbb{C} \mathbb{S}\left(U, \frac{1}{k} \mathcal{B}\right)$, which implies the existence of the commutative diagram 44.2

Case $n=46$.

The variety $X$ is a hypersurface in $\mathbb{P}(1,1,3,7,10)$ of degree 21 , the singularities of $X$ consist of the point $P$ that is a singularity of type $\frac{1}{10}(1,3,7)$. There is a commutative diagram

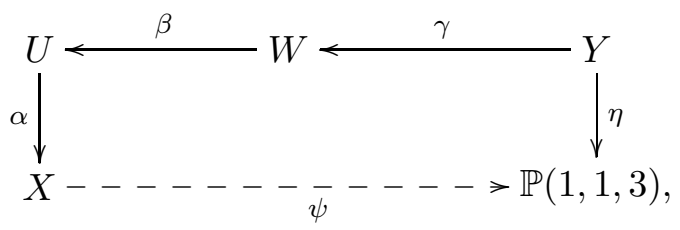

where $\alpha$ is the blow up of $P$ with weights $(1,3,7), \beta$ is the blow up with weights $(1,3,4)$ of the singular point of $U$ that is a singularity of type $\frac{1}{7}(1,3,4), \gamma$ is the blow up with weights $(1,1,3)$ of the singular point of $W$ that is a singularity of type $\frac{1}{4}(1,1,3)$, and $\eta$ is an elliptic fibration.

It follows from Proposition 3.7 that $\mathbb{C} S\left(X, \frac{1}{k} \mathcal{M}\right)=\{P\}$.

Let $\mathcal{D}$ be the proper transform of the linear system $\mathcal{M}$ on the variety $W$. Then it follows from the proof of Proposition 22.1 that $\mathcal{D} \sim_{\mathbb{Q}}-k K_{W}$, and the set $\mathbb{C} \mathbb{S}\left(W, \frac{1}{k} \mathcal{D}\right)$ does not contain subvarieties of the variety $W$ that are not contained in the exceptional divisor of $\beta$. 
The set $\mathbb{C} S\left(W, \frac{1}{k} \mathcal{D}\right)$ is not empty, because the divisor $-K_{W}$ is nef and big. Therefore, we can apply the arguments of the proof of Proposition 22.1 the the log pair $\left(W, \frac{1}{k} \mathcal{D}\right)$, which implies that the set $\mathbb{C} \mathbb{S}\left(W, \frac{1}{k} \mathcal{D}\right)$ contains the singular point of $W$ that is a singularity of type $\frac{1}{4}(1,1,3)$.

The existence of the commutative diagram 44.2 is implied by Theorem 2.2

Case $n=50$.

The variety $X$ is a hypersurface in $\mathbb{P}(1,1,3,7,11)$ of degree 22 , the singularities of $X$ consist of the point $P_{1}$ that is a quotient singularity of type $\frac{1}{3}(1,1,2)$, and the point $P_{2}$ that is a quotient singularity of type $\frac{1}{7}(1,3,4)$. The equality $-K_{X}^{3}=2 / 21$ holds. There is a commutative diagram

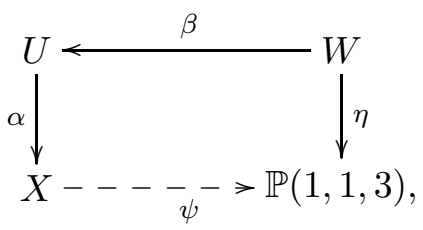

where $\alpha$ is the blow up of $P_{2}$ with weights $(1,3,7), \beta$ is the blow up with weights $(1,1,3)$ of the singular point of $U$ that is a singularity of type $\frac{1}{4}(1,1,3)$, and $\eta$ is an elliptic fibration.

The proof of Proposition 22.1 implies the existence of the diagram 44.2

Case $n=54$.

The variety $X$ is a hypersurface in $\mathbb{P}(1,1,6,8,9)$ of degree 24 , whose singularities consist of the point $P_{1}$ type $\frac{1}{2}(1,1,1)$, the point $P_{2}$ of type $\frac{1}{3}(1,1,2)$, the point $P_{3}$ of type $\frac{1}{9}(1,1,8)$.

There is a commutative diagram

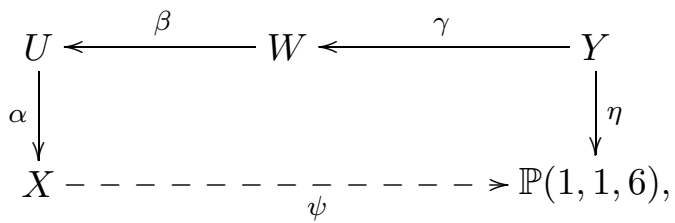

where $\alpha$ is the blow up of $P_{3}$ with weights $(1,1,8), \beta$ is the blow up with weights $(1,1,7)$ of the singular point of $U$ that is a singularity of type $\frac{1}{8}(1,1,7), \gamma$ is the blow up with weights $(1,1,3)$ of the singular point of $W$ that is a singularity of type $\frac{1}{7}(1,1,6)$, and $\eta$ is an elliptic fibration.

The existence of the diagram 44.2 follows from the proof of Proposition 14.1

Case $n=55$.

The variety $X$ is a hypersurface in $\mathbb{P}(1,2,3,7,12)$ of degree 24 , whose singularities consist of the points $P_{1}$ and $P_{2}$ of type $\frac{1}{2}(1,1,1)$, the points $P_{3}$ and $P_{4}$ of type $\frac{1}{3}(1,1,2)$, and the singular point $P_{5}$ of type $\frac{1}{7}(1,2,5)$. There is a commutative diagram

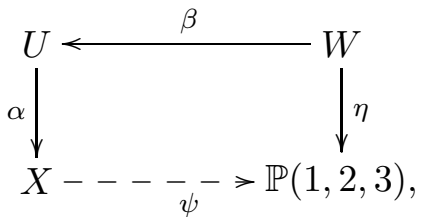

where $\alpha$ is the blow up of $P_{5}$ with weights $(1,2,5), \beta$ is the blow up with weights $(1,2,3)$ of the singular point of $U$ that is a singularity of type $\frac{1}{5}(1,2,3)$, and $\eta$ is an elliptic fibration.

It follows from Proposition 3.7 that $\mathbb{C S}\left(X, \frac{1}{k} \mathcal{M}\right)=\left\{P_{5}\right\}$.

Let $\mathcal{B}$ be the proper transform of the linear system $\mathcal{M}$ on the variety $U$, and $P_{6}$ and $P_{7}$ be the singular points of $U$ that are singularities of types $\frac{1}{2}(1,1,1)$ and $\frac{1}{5}(1,2,3)$ contained in the exceptional divisor of $\alpha$ respectively. Then $\mathbb{C S}\left(U, \frac{1}{k} \mathcal{B}\right) \subseteq\left\{P_{6}, P_{7}\right\}$ by Lemmas [2.1] 2.3 and 2.4.

The existence of the diagram 44.2 follows from Theorem 2.2 in the case when $P_{7} \in \mathbb{C} S\left(U, \frac{1}{k} \mathcal{B}\right)$, which implies that we may assume that the set $\mathbb{C S}\left(U, \frac{1}{k} \mathcal{B}\right)$ consists of the point $P_{6}$.

The hypersurface $X$ can be given by the equation

$$
t^{3} z+t^{2} f_{10}(x, y, z, w)+t f_{17}(x, y, z, w)+f_{24}(x, y, z, w)=0 \subset \operatorname{Proj}(\mathbb{C}[x, y, z, t, w]),
$$


where $\operatorname{wt}(x)=1, \operatorname{wt}(y)=2, \operatorname{wt}(z)=3, \operatorname{wt}(t)=7, \operatorname{wt}(w)=12$, and $f_{i}(x, y, z, w)$ is a sufficiently general quasihomogeneous polynomial of degree $i$. Let $\gamma: W \rightarrow U$ be the weighted blow up of the point $P_{6}$ with weights $(1,1,1), \mathcal{H}$ be the proper transform of the linear system $\mathcal{M}$ on the variety $W, \mathcal{P}$ be the proper transform on the variety $W$ of the pencil of surfaces that are cut on the hypersurface $X$ by the equations $\lambda x^{3}+\mu z=0$, where $(\lambda, \mu) \in \mathbb{P}^{1}$, and $D$ be a sufficiently general surface of the pencil $\mathcal{P}$. Then the base locus of the pencil $\mathcal{P}$ consists of the irreducible curves $C, L$ and $\Delta$ such that $\alpha \circ \gamma(C)$ is the base curve of $\left|-3 K_{X}\right|$, the curve $\gamma(L)$ is contained in the exceptional divisor of $\alpha$, and the curve $\Delta$ is contained in the exceptional divisor of $\gamma$.

The surface $D$ is normal, the intersection form of the curves $L$ and $C$ on the surface $D$ is negatively defined, but $\left.\mathcal{H}\right|_{D} \sim_{\mathbb{Q}} k C+k L$, which is impossible by Lemmas 2.10 and 2.8.

Case $n=61$.

The variety $X$ is a hypersurface in $\mathbb{P}(1,4,5,7,9)$ of degree 25 , the singularities of $X$ consist of the point $P_{1}$ that is a quotient singularity of type $\frac{1}{4}(1,1,3)$, the point $P_{2}$ that is a quotient singularity of type $\frac{1}{7}(1,2,5)$, and the point $P_{3}$ that is a quotient singularity of type $\frac{1}{9}(1,4,5)$.

The indeterminacies the map rational $\psi$ is resolved by the weighted blow ups of the singular points $P_{2}$ and $P_{3}$ with weights $(1,2,5)$, and $(1,4,5)$ respectively.

It follows from Proposition 3.7 that $\mathbb{C S}\left(X, \frac{1}{k} \mathcal{M}\right) \subseteq\left\{P_{2}, P_{3}\right\}$, the proof of Lemma 36.2 implies that $\mathbb{C} \mathbb{S}\left(X, \frac{1}{k} \mathcal{M}\right)=\left\{P_{2}, P_{3}\right\}$, and the existence of the diagram 44.2 follows from Theorem 2.2 ,

Case $n=62$.

The variety $X$ is a hypersurface in $\mathbb{P}(1,1,5,7,13)$ of degree 26 , the singularities of $X$ consist of the point $P_{1}$ that is a quotient singularity of type $\frac{1}{5}(1,2,3)$, and the point $P_{2}$ that is a singularity of type $\frac{1}{7}(1,1,6)$. The equality $-K_{X}^{3}=2 / 35$ holds. There is a commutative diagram

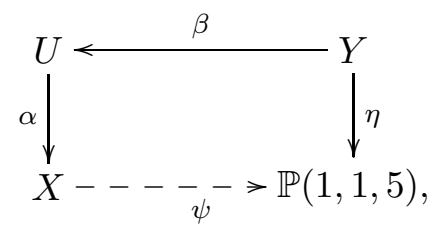

where $\alpha$ is the weighted blow up of the point $P_{2}$ with weights $(1,1,6), \beta$ is the weighted blow up with weights $(1,1,5)$ of the singular point of the variety $U$ that is a quotient singularity of type $\frac{1}{6}(1,1,5)$, and $\eta$ is an elliptic fibration.

It follows from Proposition 3.7 that $\mathbb{C S}\left(X, \frac{1}{k} \mathcal{M}\right) \subseteq\left\{P_{1}, P_{2}\right\}$. Therefore, the existence of the commutative diagram 44.2 follows from the proof of Proposition 31.1

Case $n=63$.

The variety $X$ is a hypersurface in $\mathbb{P}(1,2,3,8,13)$ of degree 26 , the singularities of the hypersurface $X$ consist of the points $P_{1}, P_{2}$ and $P_{3}$ that are quotient singularities of type $\frac{1}{2}(1,1,1)$, the point $P_{4}$ that is a quotient singularity of type $\frac{1}{3}(1,1,2)$, and the point $P_{5}$ that is a singularity of type $\frac{1}{8}(1,3,5)$. The equality $-K_{X}^{3}=1 / 24$ holds.

There is a commutative diagram

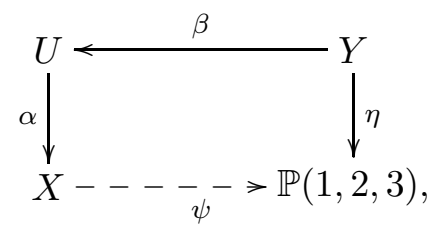

where $\alpha$ is the weighted blow up of the point $P_{5}$ with weights $(1,3,5)$, and $\beta$ is the weighted blow up with weights $(1,2,3)$ of the singular point of the variety $U$ that is a quotient singularity of type $\frac{1}{5}(1,2,3)$ contained in the exceptional divisor of $\alpha$, and $\eta$ is an elliptic fibration.

It follows from Proposition 3.7 that $\mathbb{C S}\left(X, \frac{1}{k} \mathcal{M}\right)=\left\{P_{5}\right\}$.

Let $\mathcal{B}$ be the proper transform of $\mathcal{M}$ on $U$, and $P_{6}$ and $P_{7}$ be the singular points of $U$ that are quotient singularities of types $\frac{1}{5}(1,2,3)$ and $\frac{1}{2}(1,1,1)$ contained in the exceptional divisor of the morphism $\alpha$ respectively. Then it follows from Lemma 2.3 that the set $\mathbb{C S}\left(U, \frac{1}{k} \mathcal{B}\right)$ contains either the point $P_{6}$, or the point $P_{7}$. 
Suppose that the set $\mathbb{C} S\left(U, \frac{1}{k} \mathcal{B}\right)$ contains the point $P_{7}$. Let $\gamma: W \rightarrow U$ be the weighted blow up of the point $P_{7}$ with weights $(1,1,1)$, and $\mathcal{H}$ and $\mathcal{P}$ be the proper transforms of the linear system $\mathcal{M}$ and the pencil $\left|-2 K_{X}\right|$ on the variety $W$ respectively. Then the base locus of the pencil $\mathcal{P}$ consists of the irreducible curves $C$ and $L$ such that the curve $\alpha \circ \gamma(C)$ is the unique curve in the base locus of the pencil $\left|-2 K_{X}\right|$, and the curve $\gamma(L)$ is contained in the exceptional divisor of the birational morphism $\alpha$. Moreover, the surface $D$ is normal, and the equalities

$$
C \cdot C=-\frac{5}{12}, L \cdot L=-\frac{7}{20}, C \cdot L=\frac{1}{4}
$$

hold on $D$. Hence, the intersection form of the curves $L$ and $C$ on the surface $D$ is negatively defined. On the other hand, the equivalence $\left.\mathcal{H}\right|_{D} \sim_{\mathbb{Q}} k C+k L$ holds on the surface $D$, which contradicts Lemmas 2.10 and 2.8 .

Therefore, the set $\mathbb{C S}\left(U, \frac{1}{k} \mathcal{B}\right)$ contains the point $P_{6}$. Now the claim of Theorem 2.2 easily implies the existence of the commutative diagram 44.2

Case $n=67$.

The variety $X$ is a hypersurface in $\mathbb{P}(1,1,4,9,14)$ of degree 28 , and the singularities of the hypersurface $X$ consist of the point $P_{1}$ that is a quotient singularity of type $\frac{1}{2}(1,1,1)$, and the point $P_{2}$ that is a quotient singularity of type $\frac{1}{9}(1,4,5)$. The equality $-K_{X}^{3}=1 / 18$ holds.

It follows from Proposition 3.7 that $\mathbb{C} \mathbb{S}\left(X, \frac{1}{k} \mathcal{M}\right)=\left\{P_{2}\right\}$.

There is a commutative diagram

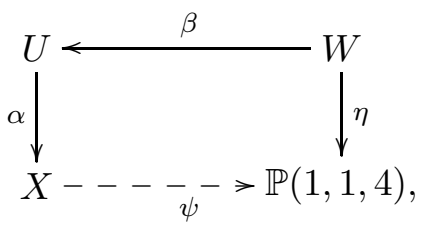

where $\alpha$ is the weighted blow up of the point $P_{2}$ with weights $(1,4,5), \beta$ is the weighted blow up with weights $(1,1,4)$ of the singular point of $U$ that is quotient singularity of type $\frac{1}{5}(1,1,4)$, and $\eta$ is an elliptic fibration.

The existence of the commutative diagram 44.2 follows from the proof of Proposition 22.1

Case $n=69$.

The variety $X$ is a hypersurface in $\mathbb{P}(1,4,6,7,11)$ of degree 28 , and the singularities of the hypersurface $X$ consist of the points $P_{1}$ and $P_{2}$ that are quotient singularities of type $\frac{1}{2}(1,1,1)$, and the points $P_{3}$ and $P_{4}$ that are quotient singularities of types $\frac{1}{6}(1,1,5) \frac{1}{11}(1,4,7)$ respectively.

The equality $-K_{X}^{3}=1 / 66$ holds, and there is a commutative diagram

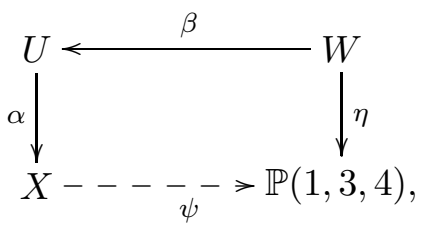

where $\alpha$ is the weighted blow up of the point $P_{4}$ with weights $(1,4,7), \beta$ is the weighted blow up with weights $(1,3,4)$ of the singular point of type $\frac{1}{7}(1,3,4)$, and $\eta$ is an elliptic fibration.

It follows from Proposition 3.7 that $\mathbb{C S}\left(X, \frac{1}{k} \mathcal{M}\right)=\left\{P_{4}\right\}$.

Let $\mathcal{B}$ be the proper transform of $\mathcal{M}$ on $U$, and $P_{5}$ and $P_{6}$ be the singular points of $U$ that are quotient singularities of types $\frac{1}{7}(1,3,4)$ and $\frac{1}{4}(1,1,3)$ contained in the exceptional divisor of the morphism $\alpha$ respectively. Then it follows from Lemmas 2.1 and 2.3 that $\mathbb{C S}\left(U, \frac{1}{k} \mathcal{B}\right)$ contains either the point $P_{5}$, or the point $P_{6}$.

It follows from the proof of Lemma 28.6 that the set $\mathbb{C} \mathbb{S}\left(U, \frac{1}{k} \mathcal{B}\right)$ does not contain the singular point $P_{6}$. Thus, the set $\mathbb{C S}\left(U, \frac{1}{k} \mathcal{B}\right)$ contains the point $P_{5}$.

The existence of the commutative diagram 44.2 follows from Theorem 2.2

Case $n=71$. 
The variety $X$ is a hypersurface in $\mathbb{P}(1,1,6,8,15)$ of degree 30 , whose singularities consist of the point $P_{1}, P_{2}$ and $P_{3}$ that are singularities of type $\frac{1}{2}(1,1,1), \frac{1}{3}(1,1,2)$ and $\frac{1}{8}(1,1,7)$ respectively. The equality $-K_{X}^{3}=1 / 24$ holds. There is a commutative diagram

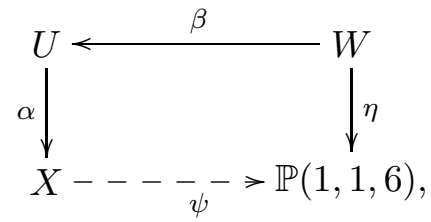

where $\alpha$ is the weighted blow up of the point $P_{3}$ with weights $(1,1,7), \beta$ is the weighted blow up with weights $(1,1,6)$ of the singular point of $U$ that is contained in the exceptional divisor of $\alpha$, and $\eta$ is an elliptic fibration. It follows from Proposition 3.7 that $\mathbb{C S}\left(X, \frac{1}{k} \mathcal{M}\right)=\left\{P_{3}\right\}$.

Let $\mathcal{D}$ be the proper transform of $\mathcal{M}$ on $U$. Then $\mathcal{D} \sim_{\mathbb{Q}}-k K_{U}$ by Theorem 2.2 and it follows from Lemmas 2.1 and 2.3 that the set $\mathbb{C S}\left(U, \frac{1}{k} \mathcal{D}\right)$ contains the singular point of $U$ that is contained in the exceptional divisor of the morphism $\alpha$.

The existence of the commutative diagram 44.2 is implied by Theorem 2.2

Case $n=76$.

The variety $X$ is a hypersurface in $\mathbb{P}(1,5,6,8,11)$ of degree 30 , whose singularities consist of the point $P_{1}, P_{2}$ and $P_{3}$ that are singularities of type $\frac{1}{2}(1,1,1), \frac{1}{8}(1,3,5)$ and $\frac{1}{11}(1,5,6)$ respectively. The indeterminacies of the projection $\psi$ are resolved by the weighted blow up of the singular points $P_{2}$ and $P_{3}$ with weights $(1,3,5)$ and $(1,5,6)$ respectively.

The proofs of Lemmas 29.3 and 36.2 implies that $\mathbb{C S}\left(X, \frac{1}{k} \mathcal{M}\right)=\left\{P_{2}, P_{3}\right\}$, which implies the existence of the commutative diagram 44.2 due to Theorem 2.2

Case $n=77$.

The variety $X$ is a hypersurface in $\mathbb{P}(1,2,5,9,16)$ of degree 32 , the singularities of $X$ consist of the points $P_{1}$ and $P_{2}$ that are quotient singularities of type $\frac{1}{2}(1,1,1)$, the point $P_{3}$ that is a quotient singularity of type $\frac{1}{5}(1,1,4)$, the point $P_{4}$ that is a quotient singularity of type $\frac{1}{9}(1,2,7)$.

The equality $-K_{X}^{3}=1 / 45$ holds, and there is a commutative diagram

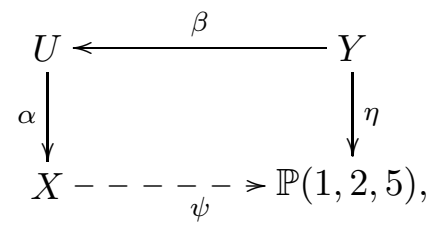

where $\alpha$ is the blow up of $P_{4}$ with weights $(1,2,7), \beta$ is the blow up with weights $(1,2,5)$ of the singular point of $U$ that is a singularity of type $\frac{1}{7}(1,2,5)$, and $\eta$ is an elliptic fibration.

It follows from Proposition 3.7 that $\mathbb{C S}\left(X, \frac{1}{k} \mathcal{M}\right)=\left\{P_{4}\right\}$.

Let $\mathcal{B}$ be the proper transform the linear system $\mathcal{M}$ on the variety $U$, and $P_{5}$ and $P_{6}$ be the singular points of $U$ that are singularities of types $\frac{1}{7}(1,2,5)$ and $\frac{1}{2}(1,1,1)$ contained in the exceptional divisor of $\alpha$ respectively. Then $\mathbb{C S}\left(U, \frac{1}{k} \mathcal{B}\right) \cap\left\{P_{5}, P_{6}\right\} \neq \varnothing$ by Lemmas 2.1 and 2.3 .

Suppose that the set $\mathbb{C} S\left(U, \frac{1}{k} \mathcal{B}\right)$ contains the point $P_{6}$. Let $\gamma: W \rightarrow U$ be the weighted blow up of the singular point $P_{5}$ with weights $(1,1,1), \mathcal{H}$ and $\mathcal{D}$ be the proper transforms of the linear systems $\mathcal{M}$ and $\left|-16 K_{X}\right|$ on the variety $W$ respectively, $D$ be a general surface of the linear system $\mathcal{D}$, and $H_{1}$ and $H_{2}$ be general surfaces of the linear system $\mathcal{H}$. Then the base locus of the linear system $\mathcal{D}$ does not contain curves. In particular, the divisor $D$ is nef, but $D \cdot H_{1} \cdot H_{1}<0$.

Therefore, the set $\mathbb{C S}\left(U, \frac{1}{k} \mathcal{B}\right)$ contains the point $P_{5}$. Now the claim of Theorem 2.2 implies the existence of the commutative diagram 44.2 .

Case $n=83$.

The variety $X$ is a general hypersurface in $\mathbb{P}(1,3,4,11,18)$ of degree 36 , and the singularities of the hypersurface $X$ consist of the point $P_{1}$ that is a quotient singularity of type $\frac{1}{2}(1,1,1)$, the points $P_{2}$ and $P_{3}$ that are quotient singularities of type $\frac{1}{3}(1,1,2)$, the point $P_{4}$ that is a quotient 
singularity of type $\frac{1}{11}(1,4,7)$. There is a commutative diagram

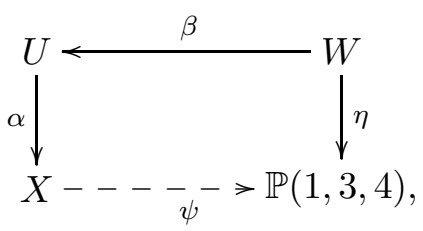

where $\alpha$ is the weighted blow up of the point $P_{4}$ with weights $(1,4,7), \beta$ is the weighted blow up with weights $(1,3,4)$ of the singular point of $U$ that is a quotient singularity of type $\frac{1}{7}(1,3,4)$, and $\eta$ is an elliptic fibration. It follows from Proposition 3.7 that $\mathbb{C} \mathbb{S}\left(X, \frac{1}{k} \mathcal{M}\right)=\left\{P_{4}\right\}$.

Let $\mathcal{B}$ be the proper transform of $\mathcal{M}$ on $U$, and $P_{5}$ and $P_{6}$ be the singular points of $U$ that are singularities of types $\frac{1}{7}(1,3,4)$ and $\frac{1}{4}(1,1,3)$ contained in the exceptional divisor of the morphism $\alpha$ respectively. Then the proof of Proposition 25.1 implies that $\mathbb{C} \mathbb{S}\left(U, \frac{1}{k} \mathcal{B}\right)$ does not contain the singular point $P_{6}$. Thus, the set $\mathbb{C S}\left(U, \frac{1}{k} \mathcal{B}\right)$ contains the point $P_{5}$ by Lemmas 2.1] and 2.3] and the existence of the diagram 44.2 follows from Theorem 2.2

Case $n=85$.

The variety $X$ is a hypersurface in $\mathbb{P}(1,3,5,11,19)$ of degree 38 , the singularities of $X$ consist of the point $P_{1}$ that is a quotient singularity of type $\frac{1}{3}(1,1,2)$, the point $P_{2}$ that is a quotient singularity of type $\frac{1}{5}(1,1,4)$, and the point $P_{3}$ that is a quotient singularity of type $\frac{1}{11}(1,3,8)$.

The equality $-K_{X}^{3}=2 / 165$ holds, and there is a commutative diagram

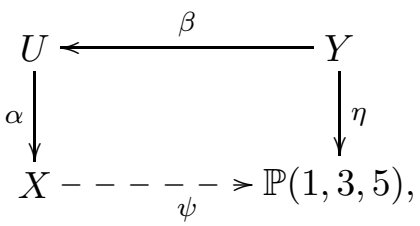

where $\alpha$ is the weighted blow up of the point $P_{3}$ with weights $(1,3,8), \beta$ is the weighted blow up with weights $(1,3,5)$ of the singular point of $U$ that is a singularity of type $\frac{1}{8}(1,3,5)$, and $\eta$ is an elliptic fibration. It follows from Proposition 3.7 that $\mathbb{C} \mathbb{S}\left(X, \frac{1}{k} \mathcal{M}\right)=\left\{P_{3}\right\}$.

Let $\mathcal{B}$ be the proper transform of $\mathcal{M}$ on $U$, and $P_{4}$ and $P_{5}$ be the singular points of $U$ that are quotient singularities of types $\frac{1}{8}(1,3,5)$ and $\frac{1}{3}(1,1,2)$ contained in the exceptional divisor of the morphism $\alpha$ respectively. Then $\mathbb{C S}\left(U, \frac{1}{k} \mathcal{B}\right) \cap\left\{P_{4}, P_{5}\right\} \neq \varnothing$ by Lemmas 2.1 and 2.3.

Suppose that the set $\mathbb{C} \mathbb{S}\left(U, \frac{1}{k} \mathcal{B}\right)$ contains the point $P_{5}$. Let $\gamma: W \rightarrow U$ be the weighted blow up of the singular point $P_{5}$ with weights $(1,1,2), \mathcal{H}$ and $\mathcal{D}$ be the proper transforms of the linear systems $\mathcal{M}$ and $\left|-19 K_{X}\right|$ on the variety $W$ respectively, $D$ be a general surface of the linear system $\mathcal{D}$, and $H_{1}$ and $H_{2}$ be general surfaces of the linear system $\mathcal{H}$. Then the base locus of the linear system $\mathcal{D}$ does not contain curves.

The divisor $D$ is nef. In particular, the inequality $D \cdot H_{1} \cdot H_{1} \geqslant 0$ holds, but it follows from the simple explicit computations that $D \cdot H_{1} \cdot H_{1}=-2 k^{2} / 15$, which is a contradiction.

Hence, the set $\mathbb{C S}\left(U, \frac{1}{k} \mathcal{B}\right)$ contains the singular point $P_{4}$. Thus, the existence of the commutative diagram 44.2 follows from Theorem 2.2

Case $n=91$.

The variety $X$ is a hypersurface in $\mathbb{P}(1,4,5,13,22)$ of degree 44 , the singularities of $X$ consist of the point $P_{1}$ that is a quotient singularity of type $\frac{1}{2}(1,1,1)$, the point $P_{2}$ that is a quotient singularity of type $\frac{1}{5}(1,2,3)$, the point $P_{3}$ that is a quotient singularity of type $\frac{1}{13}(1,4,9)$.

The equality $-K_{X}^{3}=1 / 130$ holds, and there is a commutative diagram

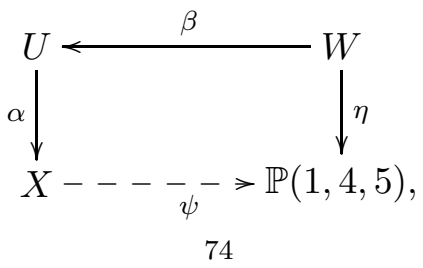


where $\alpha$ is the weighted blow up of the point $P_{3}$ with weights $(1,4,9), \beta$ is the weighted blow up with weights $(1,4,5)$ of the singular point of the variety $U$ that is a quotient singularity of type $\frac{1}{9}(1,4,5)$, and $\eta$ is an elliptic fibration.

It follows from Proposition 3.7 that $\mathbb{C S}\left(X, \frac{1}{k} \mathcal{M}\right)=\left\{P_{3}\right\}$.

Let $\mathcal{B}$ be the proper transform of $\mathcal{M}$ on $U$, and $P_{4}$ and $P_{5}$ be the singular points of $U$ that are quotient singularities of types $\frac{1}{9}(1,4,5)$ and $\frac{1}{4}(1,1,3)$ contained in the exceptional divisor of the morphism $\alpha$ respectively. Then it follows from Lemmas 2.1 and 2.3 that $\mathbb{C S}\left(U, \frac{1}{k} \mathcal{B}\right)$ contains either the point $P_{4}$, or the point $P_{5}$.

It follows from the proof of Proposition 25.1 that the set $\mathbb{C S}\left(U, \frac{1}{k} \mathcal{B}\right)$ does not contain the singular point $P_{5}$. Thus, the set $\mathbb{C S}\left(U, \frac{1}{k} \mathcal{B}\right)$ contains the singular point $P_{4}$, and the existence of the commutative diagram 44.2 follows from Theorem 2.2

The claim of Theorem 1.10 is proved.

\section{REFERENCES}

[1] I. Cheltsov, Log pairs on birationally rigid varieties Journal of Mathematical Sciences 102 (2000), 3843-3875

[2] I. Cheltsov, Anticanonical models of Fano 3-folds of degree four Sbornik: Mathematics 194 (2003), 617-640

[3] I. Cheltsov, Birationally rigid Fano varieties Russian Mathematical Surveys 60 (2005), 71-160

[4] I. Cheltsov, J. Park, Weighted Fano threefold hypersurfaces Journal fur die Reine und Angewandte Mathematik,

[5] A. Corti, Singularities of linear systems and 3-fold birational geometry L.M.S. Lecture Note Series 281 (2000), 259-312

[6] A. Corti, A. Pukhlikov, M. Reid, Fano 3-fold hypersurfaces L.M.S. Lecture Note Series 281 (2000), 175-258

[7] A. R. Iano-Fletcher, Working with weighted complete intersections L.M.S. Lecture Note Series 281 (2000), 101-173

[8] Y. Kawamata, Divisorial contractions to 3-dimensional terminal quotient singularities Higher-dimensional complex varieties (Trento, 1994), de Gruyter, Berlin (1996), 241-246

[9] Y. Kawamata, K. Matsuda, K. Matsuki, Introduction to the minimal model problem Advanced Studies in Pure Mathematics 10 (1987), 283-360

[10] J. Kollár, Singularities of pairs Proceedings of Symposia in Pure Mathematics 62 (1997), 221-287

[11] D. Ryder, Classification of elliptic and K3 fibrations birational to some $\mathbb{Q}$-Fano 3 -folds Journal of Mathematical Sciences, The University of Tokyo, 13 (2006), 13-42

[12] D. Ryder, The Curve Exclusion Theorem for elliptic and K3 fibrations birational to Fano 3-fold hypersurfaces arXiv:math.AG/0606177 (2006)

[13] V.Shokurov, 3-fold log flips Russian Academy of Sciences. Izvestiya. Mathematics 40 (1993), 95-202

Steklov Institute of Mathematics

8 Gubkin street, Moscow 117966

Russia

cheltsov@yahoo.com
School of Mathematics The University of Edinburgh Kings Buildings, Mayfield Road Edinburgh EH9 3JZ, UK

I.Cheltsov@ed.ac.uk 